City University of New York (CUNY)

CUNY Academic Works

1999

\title{
The Contribution of Interaural Intensity Differences to the Horizontal Auditory Localization of Narrow Bands of Noise
}

Matthew H. Bakke

Graduate Center, City University of New York

\section{How does access to this work benefit you? Let us know!}

More information about this work at: https://academicworks.cuny.edu/gc_etds/1770

Discover additional works at: https://academicworks.cuny.edu

This work is made publicly available by the City University of New York (CUNY).

Contact: AcademicWorks@cuny.edu 


\section{INFORMATION TO USERS}

This manuscript has been reproduced from the microfilm master. UMI films the text directly from the original or copy submitted. Thus, some thesis and dissertation copies are in typewriter face, while others may be from any type of computer printer.

The quality of this reproduction is dependent upon the quality of the copy submitted. Broken or indistinct print, colored or poor quality illustrations and photographs, print bleedthrough, substandard margins, and improper alignment can adversely affect reproduction.

In the unlikely event that the author did not send UMI a complete manuscript and there are missing pages, these will be noted. Also, if unauthorized copyright material had to be removed, a note will indicate the deletion.

Oversize materials (e.g., maps, drawings, charts) are reproduced by sectioning the original, beginning at the upper left-hand comer and continuing from left to right in equal sections with small overlaps. Each original is also photographed in one exposure and is included in reduced form at the back of the book.

Photographs included in the original manuscript have been reproduced xerographically in this copy. Higher quality $6^{n} \times 9^{n}$ black and white photographic prints are available for any photographs or illustrations appearing in this copy for an additional charge. Contact UMI directly to order.

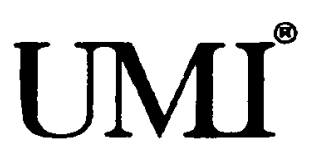

Bell \& Howell Information and Leaming 300 North Zeeb Road, Ann Arbor, MI 48106-1346 USA $800-521-0600$ 
Reproduced with permission of the copyright owner. Further reproduction prohibited without permission. 
THE CONTRIBUTION OF INTERAURAL INTENSITY DIFFERENCES TO THE HORIZONTAL AUDITORY LOCALIZATION OF NARROW BANDS OF NOISE

\section{By}

MATTHEW H. BAKKE

A dissertation submitted to the Graduate Faculty in Speech and Hearing Sciences in partial fulfillment of the requirements for the degree of Doctor of Philosophy, The City University of New York.

1999 


\section{Copyright 1999 by} Bakke, Matthew $\mathrm{H}$.

All rights reserved.

\section{UMI Microform 9946136}

Copyright 1999, by UMI Company. All rights reserved.

This microform edition is protected against unauthorized copying under Title 17, United States Code.

\section{UMI \\ 300 North Zeeb Road \\ Ann Arbor, MII 48103}


(C) 1999

MATTHEW H. BAKKE

All Rights Reserved

Reproduced with permission of the copyright owner. Further reproduction prohibited without permission. 
This manuscript has been read and accepted for the Graduate Faculty in Speech and Hearing Sciences in satisfaction of the dissertation requirement for the degree of Doctor of Philosophy.

[signature]
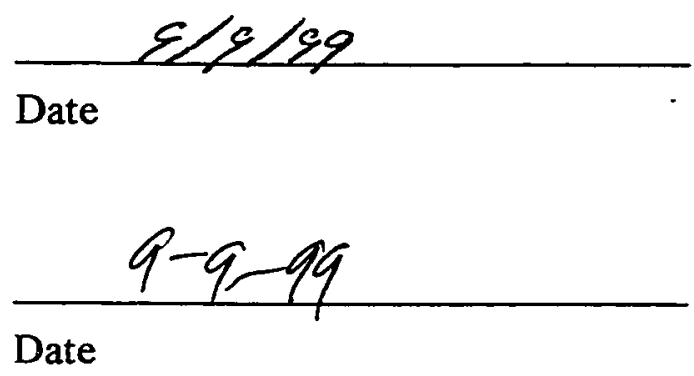

\section{Chair of Examining Committee}

[signature]

Executive Officer

Arthur Boothroyd

Stanley Gelfand

Arlene C. Neuman

Supervisory Committee

THE CITY UNIVERSITY OF NEW YORK 


\begin{abstract}
THE CONTRIBUTION OF INTERAURAL INTENSITY DIFFERENCES TO THE HORIZONTAL AUDITORY LOCALIZATION OF NARROW BANDS OF NOISE
\end{abstract}

\title{
By
}

\section{MATTHEW H. BAKKE}

Advisor: Distinguished Professor Harry Levitt

Brief bursts of third-octave bands of noise (center frequencies at $0.5,1.0,2.0$ and $4.0 \mathrm{kHz})$ and band pass noises with different degrees of low-frequency content $(0.5$ to 4.0 $\mathrm{kHz}, 1.0$ to $4.0 \mathrm{kHz}$ and 2.0 to $4.0 \mathrm{kHz}$ ) were recorded binaurally from 17 different horizontal locations (90 degrees on the left to 90 degrees on the right in 11.25 degree steps) one meter from the ears of an anthropomorphic mannequin (KEMAR) in an anechoic room and a reverberant room. The recorded sounds were processed by attenuating or removing interaural intensity differences and presented to five normally hearing subjects through insert transducers (ER-3A) in a sound-source identification task. The localization accuracy of the subjects for unprocessed signals was similar to that reported in the literature for free-field listening. Auditory localization performance was not significantly degraded by reducing interaural intensity difference cues to $50 \%$ of their original value in $\mathrm{dB}$. However, attenuating interaural intensity differences by $100 \%$ degraded localization performance by introducing a bias toward the center. The effect was frequency dependent, with no effect for a $0.5 \mathrm{kHz}$ third octave band. Some asymmetries in localization performance were observed. Localization accuracy was similar for signals recorded in a reverberant room as for those recorded in an anechoic room. 


\section{Acknowledgements}

This dissertation is dedicated to the memory of John and Margaret Meehan. Without their abounding love, generosity and good humor, this work would not have been possible.

I have many people to thank for the completion of this work, but none more than my wife, Maureen, who has patiently and faithfully stood by me during all the years of its preparation. Likewise my children: Maggie, Matt Jr. and Mary Ann, who have sacrificed so much for me, and never complained.

I thank my parents, Hans and Georgiana Bakke, who taught me to finish what I started and not to feel sorry for myself when there is so much to enjoy in the world.

Thank you, too, to all of my friends at the City University of New York: staff, students and faculty. You have been a source of strength and comfort; a place for learning and laughter. Special thanks to Harry Levitt, who helped me to be who I am, and to Shlomo Silman, who first encouraged me to undertake this task.

Finally, "thank you" to all my friends at Lexington School for the Deaf / Center for the Deaf. Your support, encouragement, kindness, patient long-suffering and help have guided me through the past six years. I will never forget you. 


\section{TABLE OF CONTENTS}

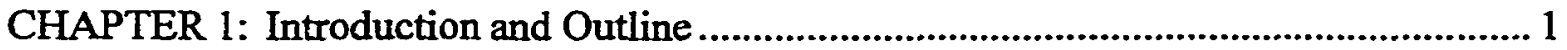

1.1 Importance of Sound Source Localization in Humans .......................................... 1

1.2 Purpose of the Experiment ..........................................................................2

1.3 Brief Description of the Experiment............................................................ 2

1.4 Outline of the Dissertation ........................................................................

CHAPTER 2: Review of the Literature ................................................................. 4

2.1 Cues for Localization............................................................................... 4

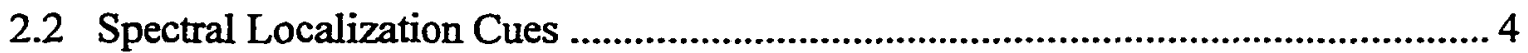

2.3 Head Movement......................................................................................... 7

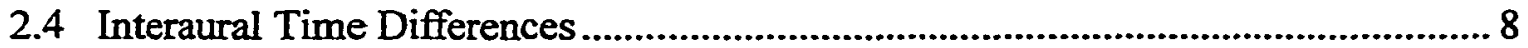

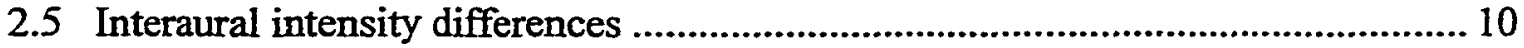

2.6 Relationship Between Interaural Time Differences and Interaural Intensity

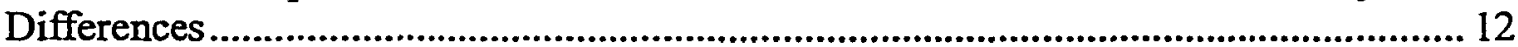

2.7 The Roles of Interaural Intensity Difference and Interaural Time Difference Cues

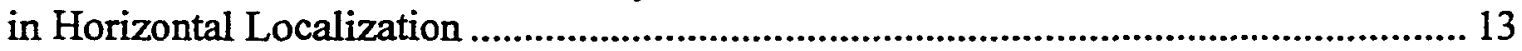

2.7.1 The Duplex Theory ........................................................................... 13

2.8 Relative Importance of Interaural Time Difference and Interaural Intensity

Difference Cues for Localization.................................................................... 15

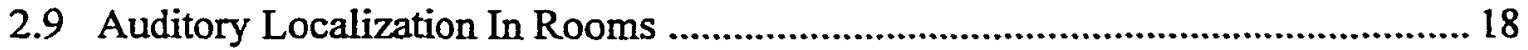

2.10 Plausibility/Reliability of Acoustic Cues for Localization ............................. 20

2.11 Research Questions and Hypotheses ...................................................... 22

2.11.1 Statement of Theory and Experimental Hypotheses.................................... 24

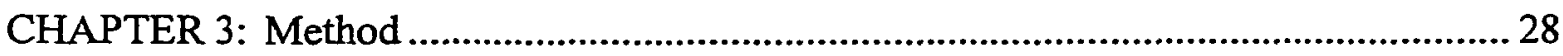

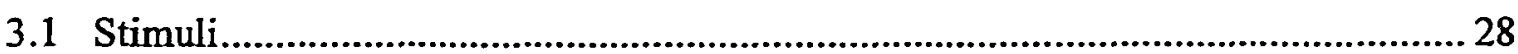

3.1.1 Construction of the Experimental Signal Sources ....................................... 28

3.1.2 Gating of the Experimental Signal Sources ................................................. 29

3.1.3 Recording the Binaural Signals ....................................................... 30

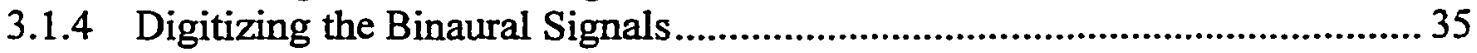

3.1.5 Processing the Binaural Signals ................................................................ 36

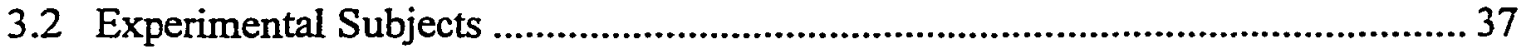

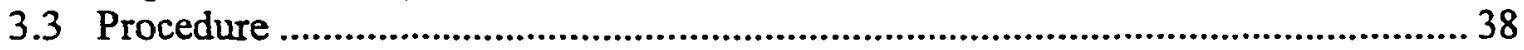

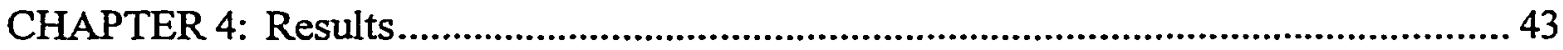

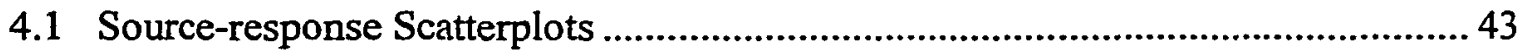

4.1.1 Left/Right Reversals .......................................................................... 45

4.2 Derivation of Measures for Quantifying Localization Error ............................. 46

4.2.1 Precision of Localization ...................................................................... 48

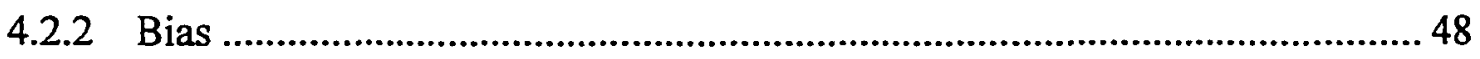

4.2.3 Accuracy of Localization ................................................................... 49

4.2.4 Summary of Measures for Quantifying Localization Error .......................... 49

4.3 Root Mean Square Error $\left(\sigma_{e}\right)$ : Accuracy of Localization ................................ 50

4.3.1 Interaural Intensity Difference Condition ................................................. 53 


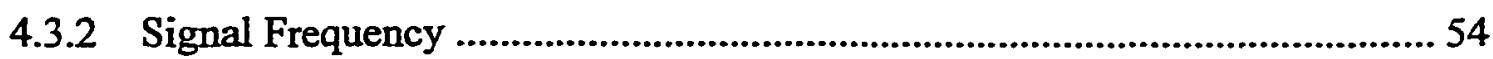

4.3.3 Interaural Intensity Difference Condition and Signal Frequency .................. 56

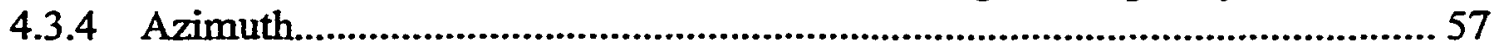

4.3.5 Azimuth and Signal Frequency.................................................................. 58

4.3.6 Azimuth and Interaural Intensity Difference Condition ................................59

4.3.7 Azimuth, Signal Frequency and Interaural Intensity Difference Condition. 61

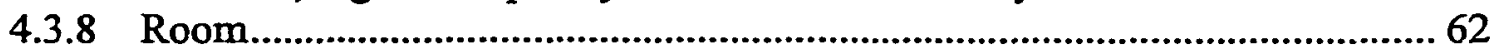

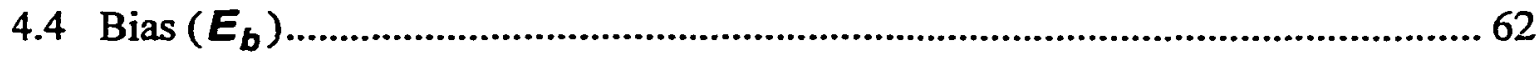

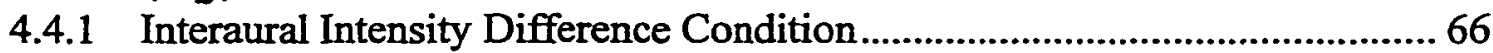

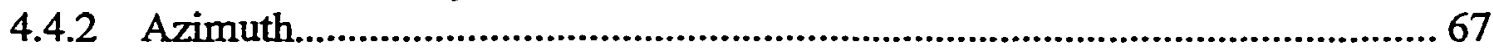

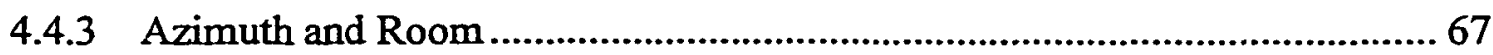

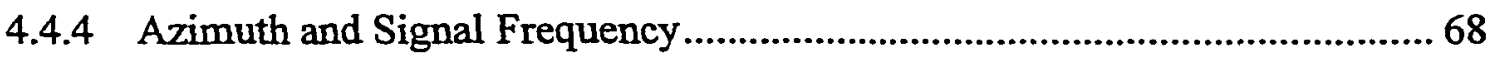

4.4.5 Azimuth and Interaural Intensity Difference Condition ................................ 70

4.4.6 Azimuth, Interaural Intensity Difference Condition and Frequency ............ 72

4.5 Test-retest standard deviation $\left(\sigma_{E}\right)$ : Precision of Localization ............................. 73

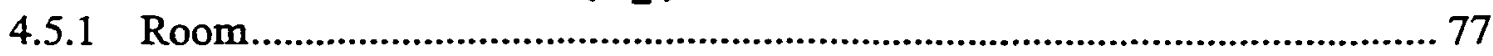

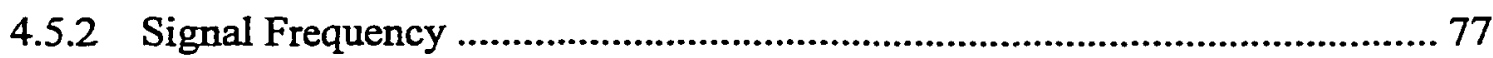

4.5.3 Interaural Intensity Difference Condition ..................................................... 78

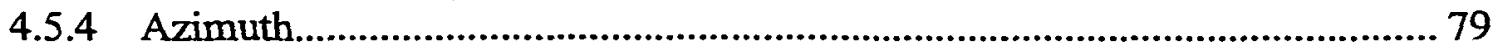

4.5.5 Azimuth and Signal Frequency................................................................ 79

4.5.6 Azimuth and Interaural Intensity Difference Condition ............................... 80

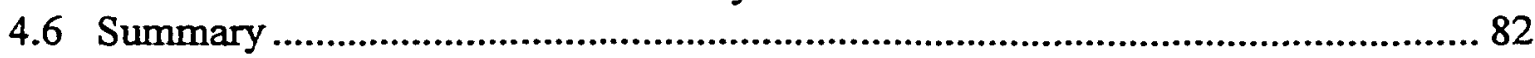

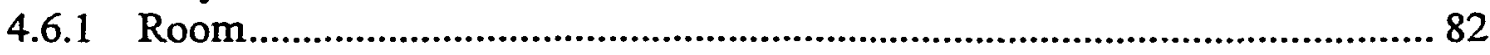

4.6.2 Interaural Intensity Difference Condition for Signals with Different

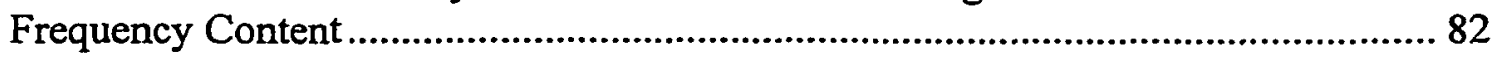

4.6.3 Azimuth and Interaural Intensity Difference Condition ................................ 83

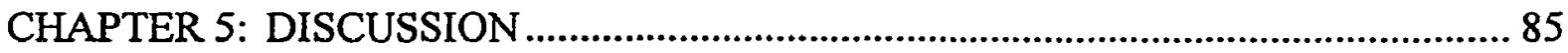

5.1 Comparison of Localization Results with Published Research. ............................8 85

5.1 .1 Overall Accuracy ..................................................................................... 85

5.1.2 Effect of Azimuth on Localization Accuracy ……..................................... 88

5.1.3 Effect of Signal Frequency on Localization Accuracy ................................. 92

5.2 Experimental Hypotheses ................................................................................. 95

5.2.1 Relative Dominance of Interaural Time Difference and Interaural Intensity

Difference Cues ................................................................................................... 95

5.2.2 Experimental Hypothesis \#1 ................................................................... 102

5.2.3 Experimental Hypothesis \#2 ................................................................... 104

5.2.4 Experimental Hypothesis \#3 ..................................................................... 106

5.2.5 Experimental Hypothesis \#4 ............................................................... 107

5.2.6 Experimental Hypotheses \#5 and \#6...................................................... 109

5.3 Implications for spatial hearing with binaural hearing aids.............................. 110

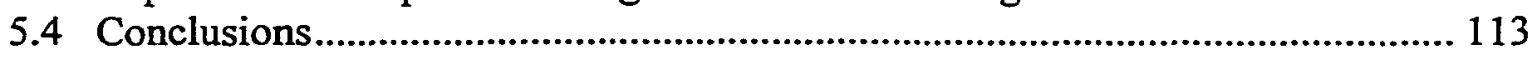

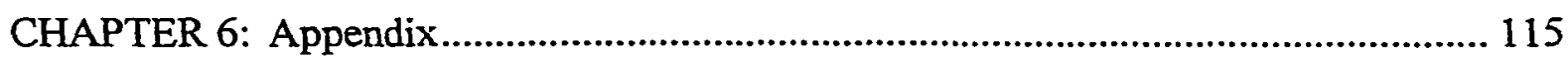

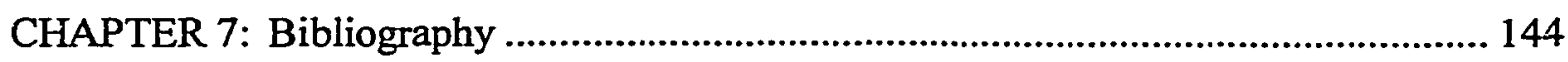




\section{LIST OF TABLES}

Table 1: The frequency limits of the experimental signals. 28

Table 2: ANOVA table of root mean square error for third-octave bands of noise. ........ 51

Table 3: ANOVA table of root mean square error for band-pass noises........................ 52

Table 4: ANOVA table of bias for third-octave bands of noise. ..................................... 64

Table 5: ANOVA table of bias for band-pass noises.

Table 6: ANOVA table of the test-retest standard deviation for third-octave bands of noise.

Table 7: ANOVA table of the test-retest standard deviation for band-pass noises .......... 76

Table 8: Horizontal localization errors resulting from recently published studies.

Table 9: Horizontal localization errors obtained using a pointing response method

Table 10: Comparisons Among the Results of Several Interaural Intensity Difference Manipulation Studies

Table 11: Maximum magnitudes (in $\mathrm{dB}$ ) of interaural intensity differences present in the signals used in this experiment.

Table 12: ANOVA table of the absolute value of the bias using side (left/right) as a factor, for the third-octave bands of noise.

Table 13: ANOVA table of the absolute value of the bias using side (left/right) as a factor, for the band-pass noises. 


\section{LIST OF FIGURES}

Figure 1: Theoretical values of interaural time difference for horizontal azimuths of interest, based on Kuhn (1977)............................................................................... 9

Figure 2: Theoretical interaural intensity differences for azimuths from $0^{\circ}$ to $90^{\circ}$ for

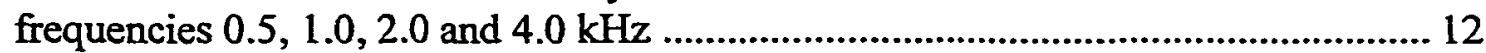

Figure 3: Instrumentation for generating the noise band stimuli...................................... 29

Figure 4: Representation of the test signal duty cycle..................................................... 30

Figure 5: Layout for binaural recordings in the anechoic room. ......................................... 31

Figure 6: Layout for binaural recordings in the minitheater................................................ 32

Figure 7: Instrumentation for recording experimental binaural stimuli............................... 33

Figure 8: Instrumentation for digitizing the binaural signals........................................... 36

Figure 9: Instrumentation for subject testing. ................................................................. 40

Figure 10: Scatterplots showing the gross effect of interaural intensity difference condition on auditory localization performance for $0.5 \mathrm{kHz}$ third-octave bands of noise.

Figure 11: Scatterplots showing the gross effect of interaural intensity difference condition on auditory localization performance for $2.0 \mathrm{kHz}$ third-octave bands of noise.

Figure 12: Number of left/right reversals for each signal type and interaural intensity difference condition.

Figure 13: Effect of interaural intensity difference condition on root mean square error. 54

Figure 14: Effect of signal frequency on root mean square error.................................... 55

Figure 15: Effect of interaural intensity difference condition and signal frequency on root mean square error................................................................................................ 56

Figure 16: Effect of sound source azimuth on root mean square error..............................58

Figure 17: Effect of signal frequency and azimuth on root mean square error .................59

Figure 18: Effects of interaural intensity difference condition and azimuth on root mean square error for third-octave bands of noise..

Figure 19: Effects of interaural intensity difference condition and azimuth on root mean

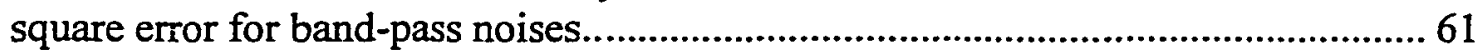

Figure 20: Effect of interaural intensity difference condition on bias.................................6 66

Figure 21: Effect of room and sound source azimuth on bias for band-pass noises......... 68

Figure 22: Effect of azimuth and signal frequency on bias for third-octave bands of noise.

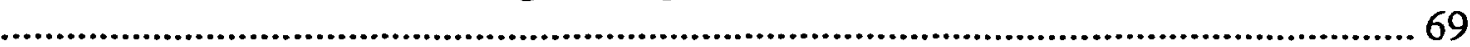

Figure 23: Effect of azimuth and signal frequency on bias for band-pass noises............. 70

Figure 24: Effect of azimuth and interaural intensity difference condition on bias for third-octave bands of noise. ..................................................................................... 71

Figure 25: Effect of azimuth and interaural intensity difference condition on bias for band-pass noises................................................................................................ 72

Figure 26: Effect of signal frequency on test-retest standard deviation for band-pass

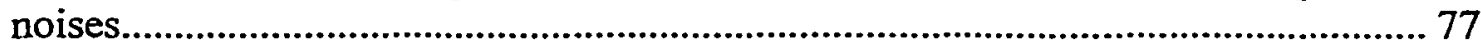

Figure 27: Effect of interaural intensity difference condition on test-retest standard deviation for band-pass noises. .................................................................................. 78

Figure 28: Effect of azimuth on test-retest standard deviation........................................ 79

Figure 29: Effect of azimuth and signal frequency on test-retest standard deviation....... 80 
Figure 30: Effect of azimuth and interaural intensity difference condition on test-retest standard deviation for the third-octave bands of noise.

Figure 31: Effect of azimuth and interaural intensity difference condition on test-retest standard deviation for the band-pass noises.

Figure 32: Relationship between azimuth and root mean square localization error $\left(\sigma_{e}\right)$ for the present experiment.

Figure 33: Relationship between azimuth and localization errors for three published studies that used broad band stimuli.

Figure 34: The contribution of precision and bias to localization accuracy for third-octave bands of noise.

Figure 35: The contribution of precision and bias to localization accuracy for band-pass noises.

Figure 36: The overall percent correct localization scores of Giguère and Abel (1993) for an absorbent and a reverberant room with the results of the present experiment for the anechoic room and minitheater.

Figure 37: Interaural intensity differences for KEMAR in an anechoic room for the signals used in the present experiment

Figure 38: Interaural intensity differences for KEMAR in the minitheater for the signals used in the present experiment.

Figure 39: The average interaural intensity differences present in each unattenuated signal used in the present experiment..

Figure 40: Relationship among accuracy, bias and precision of localization judgments under the three experimental interaural intensity difference conditions for the thirdoctave band signals.

Figure 41: Contributions of precision and bias to overall accuracy in the localization of third-octave bands for the $100 \%$ attenuated interaural intensity difference ........... 106

Figure 42: Spectrum of the $0.5 \mathrm{kHz}$ third-octave band noise used in the present experiment.

Figure 43: Spectrum of the $1.0 \mathrm{kHz}$ third-octave band noise used in the present experiment.

Figure 44: Spectrum of the $2.0 \mathrm{kHz}$ third-octave band noise used in the present experiment.

Figure 45: Spectrum of the $4.0 \mathrm{kHz}$ third-octave band noise used in the present experiment

Figure 46: Spectrum of the $0.5 \mathrm{kHz}$ to $4.0 \mathrm{kHz}$ band-pass noise used in the present experiment

Figure 47: Spectrum of the 1.0 to $4.0 \mathrm{kHz}$ band-pass noise used in the present experiment.

Figure 48: Spectrum of the $2.0 \mathrm{kHz}$ to $4.0 \mathrm{kHz}$ band-pass noise used in the present experiment.

Figure 49: Instrumentation for measuring the response of KEMAR's ears to a broad band stimulus at each loudspeaker location.

Figure 50: Scatterplot for $0.5 \mathrm{kHz}$ third-octave band noise with interaural intensity differences unattenuated.

Figure 51: Scatterplot for $0.5 \mathrm{kHz}$ third-octave band noise with interaural intensity differences attenuated by $50 \%$. 
Figure 52: Scatterplot for $0.5 \mathrm{kHz}$ third-octave band noise with interaural intensity differences attenuated by $100 \%$.

Figure 53: Scatterplot for $1.0 \mathrm{kHz}$ third-octave band noise with interaural intensity differences unattenuated.

Figure 54: Scatterplot for $1.0 \mathrm{kHz}$ third-octave band noise with interaural intensity differences attenuated by $50 \%$.

Figure 55: Scatterplot for $1.0 \mathrm{kHz}$ third-octave band noise with interaural intensity differences attenuated by $100 \%$.

Figure 56: Scatterplot for $2.0 \mathrm{kHz}$ third-octave band noise with interaural intensity differences unattenuated.

Figure 57: Scatterplot for $2.0 \mathrm{kHz}$ third-octave band noise with interaural intensity differences attenuated by $50 \%$.

Figure 58: Scatterplot for $2.0 \mathrm{kHz}$ third-octave band noise with interaural intensity differences attenuated by $100 \%$.

Figure 59: Scatterplot for $4.0 \mathrm{kHz}$ third-octave band noise with interaural intensity differences unattenuated.

Figure 60: Scatterplot for $4.0 \mathrm{kHz}$ third-octave band noise with interaural intensity differences attenuated by $50 \%$.

Figure 61: Scatterplot for $4.0 \mathrm{kHz}$ third-octave band noise with interaural intensity differences attenuated by $100 \%$.

Figure 62: Scatterplot for $0.5 \mathrm{kHz}$ to $4 \mathrm{kHz}$ band-pass noise with interaural intensity differences unattenuated.

Figure 63: Scatterplot for $0.5 \mathrm{kHz}$ to $4 \mathrm{kHz}$ band-pass noise with interaural intensity differences attenuated by $50 \%$.

Figure 64: Scatterplot for $0.5 \mathrm{kHz}$ to $4 \mathrm{kHz}$ band-pass noise with interaural intensity differences attenuated by $100 \%$.

Figure 65: Scatterplot for $1.0 \mathrm{kHz}$ to $4.0 \mathrm{kHz}$ band-pass noise with interaural intensity differences unattenuated.

Figure 66: Scatterplot for $1.0 \mathrm{kHz}$ to $4.0 \mathrm{kHz}$ band-pass noise with interaural intensity differences attenuated by $50 \%$.

Figure 67: Scatterplot for $1.0 \mathrm{kHz}$ to $4.0 \mathrm{kHz}$ band-pass noise with interaural intensity differences attenuated by $100 \%$.

Figure 68: Scatterplot for $2.0 \mathrm{kHz}$ to $4.0 \mathrm{kHz}$ band-pass noise with interaural intensity differences unattenuated.

Figure 69: Scatterplot for $2.0 \mathrm{kHz}$ to $4.0 \mathrm{kHz}$ band-pass noise with interaural intensity differences attenuated by $50 \%$.

Figure 70: Scatterplot for $2.0 \mathrm{kHz}$ to $4.0 \mathrm{kHz}$ band-pass noise with interaural intensity differences attenuated by $100 \%$.

Figure 71: Effect of interaural intensity difference condition and azimuth for the $0.5 \mathrm{kHz}$ third-octave band.

Figure 72: Effect of interaural intensity condition and azimuth for the $1.0 \mathrm{kHz}$ thirdoctave band.

Figure 73: Effect of interaural intensity difference condition and azimuth for the $2.0 \mathrm{kHz}$ third-octave band. .................................................................................. 130

Figure 74: Effect of interaural intensity difference condition and azimuth for the $4.0 \mathrm{kHz}$ third-octave band. 
Figure 75: Effect of interaural intensity difference Condition and azimuth for the $0.5 \mathrm{kHz}$

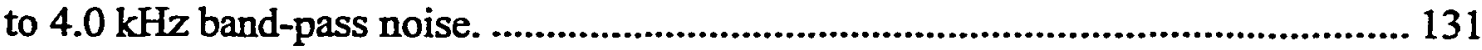

Figure 76: Effect of interaural intensity difference condition and azimuth for the $1.0 \mathrm{kHz}$

to $4.0 \mathrm{kHz}$ band-pass noise. ........................................................................ 132

Figure 77: Effect of interaural intensity difference condition and azimuth for the $2.0 \mathrm{kHz}$

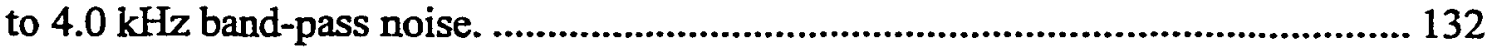

Figure 78: Effect of interaural intensity difference condition, azimuth and signal

frequency on bias for the $0.5 \mathrm{kHz}$ third-octave band noise. ............................... 133

Figure 79: Effect of interaural intensity difference condition, azimuth and signal frequency on bias for the $1.0 \mathrm{kHz}$ third-octave band noise. .............................. 133

Figure 80: Effect of interaural intensity difference condition, azimuth and signal frequency on bias for the $2.0 \mathrm{kHz}$ third-octave band noise. ................................ 134

Figure 81: Effect of interaural intensity difference condition, azimuth and signal frequency on bias the $4.0 \mathrm{kHz}$ for third-octave band noise. .................................. 134

Figure 82: Effect of interaural intensity difference condition, azimuth and signal frequency on bias for the $0.5 \mathrm{kHz}$ to $4.0 \mathrm{kHz}$ band-pass noise........................... 135

Figure 83: Effect of interaural intensity difference condition, azimuth and signal frequency on bias for the $1.0 \mathrm{kHz}$ to $4.0 \mathrm{kHz}$ band-pass noise.

Figure 84: Effect of interaural intensity difference condition, azimuth and signal frequency on bias for the $2.0 \mathrm{kHz}$ to $4.0 \mathrm{kHz}$ band-pass noise.

Figure 85: Effect of azimuth and interaural intensity difference condition for the $0.5 \mathrm{kHz}$

third-octave band of noise

Figure 86: Effect of azimuth and interaural intensity difference condition for the $1.0 \mathrm{kHz}$ third-octave band of noise

Figure 87: Effect of azimuth and interaural intensity difference condition for the $2.0 \mathrm{kHz}$

third-octave band of noise.

Figure 88: Effect of azimuth and interaural intensity difference condition for the $4.0 \mathrm{kHz}$ third-octave band of noise

Figure 89: Effect of azimuth and interaural intensity difference condition for the $0.5 \mathrm{kHz}$ to $4.0 \mathrm{kHz}$ band-pass noise.

Figure 90: Effect of azimuth and interaural intensity difference condition for the 1.0 to $4.0 \mathrm{kHz}$ band-pass noise.

Figure 91: Effect of azimuth and interaural intensity difference condition for the 2.0 to $4.0 \mathrm{kHz}$ band-pass noise. 


\section{CHAPTER 1: INTRODUCTION AND OUTLINE}

\subsection{Importance of Sound Source Localization in Humans}

Acoustic cues that help humans localize the source of a sound include interaural time differences, interaural intensity differences, binaural spectral cues and monaural spectral cues. Head movements, sound source familiarity, memory, listener expectations, cue plausibility and vision are other factors that can affect an individual's localization judgments. The most important cues for horizontal auditory localization, however, are interaural time differences and interaural intensity differences.

The ability to identify the location of a sound source is important for human listeners in many situations. Whether an individual is trying to identify the location of a predator in the forest or that of an oncoming vehicle on a city street, humans in all sorts of societies tend to rely upon hearing as a means of orienting themselves to their environment. Spatial hearing is also important for communication. A child will learn early in life to respond to the sound of his or her name by a turn of the head toward the one who is calling. An individual participating in a group discussion will rely upon spatial hearing to identify who is speaking at a given moment in time. Spatial hearing, then, has both survival value and communicative value.

Spatial hearing is no less valuable for the person with hearing loss. The wearer of hearing aids may choose a binaural fit because he or she is aware of the value of spatial hearing and expects the use of two hearing aids to preserve some level of localization ability. An important issue that confronts the audiologist dispensing binaural hearing aids is the fact that many modern hearing aids use amplitude compression to control the loudness of a hearing aid's output. The goal of amplitude compression is either to maximize the listening comfort of the hearing aid wearer and reduce the necessity for 
volume control adjustments or to compensate for the abnormal growth of loudness (recruitment) that affects many people with hearing loss. However, it is open to question whether independent adjustments to the level of signals presented to the right and left ears will result in the disruption of spatial hearing. It is reasonable to assume that interaural time difference cues will remain essentially intact for a person wearing identical hearing aids in the two ears but that interaural intensity difference cues will be affected by amplitude compression circuits when they are engaged. If this assumption is true, the answer to the question of whether binaural compression hearing aids will be likely to disrupt spatial hearing is closely related to the relative importance of interaural time differences and interaural intensity differences to sound source localization. A secondary consideration is the extent to which a given compression circuit reduces the interaural intensity difference. A 2:1 compression ratio circuit would be likely to reduce the interaural intensity difference by one-half, while a more extreme compression ratio such as 8:1 may reduce the interaural intensity difference to almost zero.

\subsection{Purpose of the Experiment}

The purpose of the present study was to evaluate the importance of interaural intensity difference cues to the accuracy and precision of auditory localization in the front medial plane. Specifically, the experiment evaluated whether interaural time difference cues are sufficient for accurate and precise horizontal auditory localization when interaural intensity differences have been reduced by $50 \%$ and when they have been reduced by $100 \%$.

\subsection{Brief Description of the Experiment}

Five subjects were asked to localize sounds that had been recorded using a Knowles Electronics Manikin for Acoustic Research (KEMAR). The interaural time 
differences and interaural intensity differences in the resulting binaural recordings were similar to those resulting from any given listener's head-related transfer function. Therefore, listeners were expected to localize them in the horizontal plane in spite of the fact that they were made on a head other their own (Wenzel, et al., 1993). The recorded sounds were presented to the ears of the subjects through custom earmolds. The subjects were seated in a quiet room at the center of a semicircular array of 17 loudspeakers and asked to name the loudspeaker from which the stimulus apparently originated. Signals were bursts of third-octave band noise centered at $0.5,1.0,2.0$ and $4.0 \mathrm{kHz}$ and bursts of band-pass noise with increasing bandwidths: 2.0 to $4.0 \mathrm{kHz}, 1.0$ to $4.0 \mathrm{kHz}$ and 0.5 to 4.0 $\mathrm{kHz}$. The signals were processed in two ways that resulted in conflicting auditory localization cues: in one condition interaural intensity differences were attenuated by $50 \%$, to one-half of their original value in $\mathrm{dB}$, and in the other they were fully attenuated $(100 \%)$. The resulting localization judgments were analyzed to evaluate the effect of attenuating interaural intensity differences on the accuracy, bias and precision of auditory localization judgments.

\subsection{Outline of the Dissertation}

Chapter 2 of this dissertation, "Review of the Literature," consists of a review of the relevant literature that led to the formulation of the experimental hypotheses. Chapter 3, "Method," describes how the experiment was prepared and conducted. In Chapter 4, "Results," the analysis of the data is described and the results of the experiment are reported. Chapter 5, "Discussion," treats the implications of the experimental findings and states the conclusions. Chapter 6, the "Appendix" contains supplementary figures and tables. All references cited in the body of the dissertation are listed in Chapter 7, the "Bibliography." 


\section{CHAPTER 2: REVIEW OF THE LITERATURE}

\subsection{Cues for Localization}

Human observers are able to localize sources of sound with great accuracy. In doing so, they may utilize both monaural and binaural auditory cues. The primary cue for monaural auditory localization is spectral: position-dependent filtering induced by the outer ear, particularly the pinna, affecting mainly high-frequency components of sound (above about $5000 \mathrm{~Hz}$ ). Binaural cues for localization include all of the position-

dependent differences between the signals received by the two ears from a single source: interaural time differences, interaural intensity differences, and interaural spectral differences (the differential effect of frequency on interaural intensity differences, particularly for frequencies above $4.0 \mathrm{kHz}$ ). Other related factors that influence auditory localization include head movements (Wightman and Kistler, 1997), source familiarity, memory, listener expectations, cue plausibility (Rakerd and Hartmann, 1985; Wenzel, et al., 1993) and vision (Shelton and Searle, 1980).

\subsection{Spectral Localization Cues}

The direction-dependent filtering of the outer ear, and the pinna in particular, results in systematic high-frequency spectral changes with changes in the position of a sound source in space. (Shaw, 1974; Blauert, 1983; Kuhn, 1987; Middlebrooks, et al, 1989). Such spectral changes may be used either monaurally or binaurally by an observer to make a localization judgment. A monaural localization judgment presumes prior knowledge of the spectral characteristics of the sound source as well as a memorized pattern of spectral changes associated with location. Binaural use of spectral cues does not presuppose prior knowledge of the stimulus since the spatial cues are derived from a comparison of the spectra at the two ears rather than a remembered spectral difference 
with source location. It does presume, however, that the observer has made perceptual associations between characteristic binaural spectral patterns and sound source locations.

Several studies have focused upon the role of pinna disparities in binaural localization. The typical procedure is to occlude the pinnae in some way and measure localization accuracy under this condition, comparing it to localization with unoccluded pinnae. Fisher and Freedman (1968) measured horizontal localization of a pulsed white noise with unoccluded pinnae, occluded pinnae and artificial pinnae. Localization was impaired in the latter two conditions if the head was restrained, but all degradation in performance disappeared when the subjects were permitted to move their heads. Musicant and Butler (1984) obtained similar results, i.e. reductions in localization accuracy, for broad band or high pass noises ( $4.0 \mathrm{kHz}$ high pass) localized in the horizontal plane with occluded pinnae. An increase in the number of front/back reversals was also observed. Localization of noise bands with no high-frequency content was not affected by occluding the pinnae. Occluding only the lead pinna also increased the number of front/back reversals, and moderately increased the localization errors. Occluding the far pinna had a similar effect, but the magnitude of the effect was much smaller.

A follow-up study by Musicant and Butler (1985) investigated the monaural and binaural horizontal localization of 11 different narrow bands of noise, $1.0 \mathrm{kHz}$ wide, with center frequencies of $4.0 \mathrm{kHz}$ to $12 \mathrm{kHz}$ in $1.0 \mathrm{kHz}$ steps. The apparent locations of the sound sources for monaural listening were largely determined by the center frequency of the noise band. Thus, a signal with a given center frequency tended to be perceived to have originated from the same location regardless of its actual point of origin. For 
binaural listening, when the signals were presented from locations near the sides or where front/back confusions occurred, the pattern of localization judgments resembled that of monaural localization judgments, i.e., they too were influenced by center frequency of the noise band. It would seem from these results that spectral cues can influence horizontal localization.

The observations of Middlebrooks (1992), however, conflicted with this observation. In a study of high-frequency narrow band localization, subjects were able to accurately localize these stimuli in the horizontal plane based upon interaural level differences, which the investigators estimated from measurements of each subjects' directional transfer function. However, systematic errors in vertical and front/back localization judgments occurred. The errors were strongly influenced by the center frequency of the stimulus noise band. Each of the narrow band experimental stimuli were found to closely resemble a spectral peak that was observed in the directional transfer function of the apparent sound source location.

There is some evidence that spectral cues are also effective for monaural localization. Jongkees and Veer (1958) reported on a group of ten subjects with unilateral deafness, of whom two exhibited "normal localization" and one exhibited "fairly good" directional hearing. Oldfield and Parker (1986) measured monaural localization for a white noise at a range of elevations $\left(-40^{\circ}\right.$ to $\left.+40^{\circ}\right)$ and azimuths $\left(0^{\circ}\right.$ to $180^{\circ}$ on the left). Subject's right ears were occluded. Horizontal localization with one ear was impaired relative to the performance that would be expected with binaural listening, but was significantly better than chance. Front/back discrimination was retained and localization for elevation was good, but not quite as good as that for binaural listening, indicating that 
pinna disparities were an additional cue (over and above monaural spectral cues) for elevation discrimination.

\subsection{Head Movement}

It has been speculated that head movement can play an important role in auditory localization because it enables the listener to sample spectral and interaural differences from more than one direction relative to the head. Thurlow and Runge (1967) conducted an experiment in which head movements consisting of tips and rotations of the head were induced in subjects while they made localization judgments for low and high-frequency bands of noise as well as for clicks. Rotation of the head provided significant localization improvements over the head-fixed condition in the horizontal plane and in particular resolved front/rear confusions for all types of signals. Permitting subjects to freely move their heads had the same effect as the experimentally controlled rotations of the head. In the investigations of pinna effects by Fisher and Freedman (1968) reported above, head movements improved localization accuracy under all pinna conditions, effectively wiping out any effect of pinna occlusion in that study. In contrast, Pollack and Rose (1967) found no advantage for head movements when localizing very brief duration clicks (15 ms). When a longer duration stimulus was used there was still no improvement in localization accuracy over the head-stationary condition when the subjects' heads were free to move.

Some preliminary results on a study of the effect of head movements in localization reported by Wightman and Kistler (1997) indicated that cues provided by head movements can be important for the resolution of front/back confusions. This research used virtual sound sources presented over headphones, and subjects were asked in one condition not to move their heads and in the other to freely move their heads if 
they felt it would facilitate their localization judgments. Head position was sensed by a magnetic tracker and the virtual synthesis algorithm was modified in real time, resulting in an apparently stable sound source location. Results for one subject were presented in which front/back confusions were virtually eliminated under the head movement condition.

\subsection{Interaural Time Differences}

Interaural time differences are readily predictable in a free-field environment and relatively frequency independent over large ranges of frequency. Kuhn $(1977,1987)$ found interaural time differences measured both on real heads and on a Knowles Electronics Manikin for Acoustic Research (KEMAR) to correspond reasonably accurately to computed theoretical values for a rigid sphere with an effective radius of about $9.3 \mathrm{~cm}$. For frequencies below about $500 \mathrm{~Hz}$, Equation 1 describes the relationship, while for frequencies above $3.0 \mathrm{kHz}$, Equation 2 applies:

$$
\begin{aligned}
& I T D \approx \frac{3 \alpha}{c} \sin \theta_{i n c} \\
& I T D \approx \frac{2 \alpha}{c} \sin \theta_{i n c}
\end{aligned}
$$

Equation 1

Equation 2

where $\alpha=$ equivalent head radius, $\mathrm{c}=$ ambient speed of sound and $\theta_{\text {inc }}=$ angle of incidence between the median plane of the head and the incident plane wave direction. For signals between $\sim 500 \mathrm{~Hz}$ and $\sim 2000 \mathrm{~Hz}$, intermediate values apply. These equations indicate that the ratio between interaural time differences at low-frequencies $(<\sim 500 \mathrm{~Hz})$ and interaural time differences at high-frequencies $(>-2000 \mathrm{~Hz})$ is about 3:2 for azimuths of $0^{\circ}$ to $60^{\circ}$. For high-frequencies, the interaural delay is a result of the fact that the sound travels toward the near ear in a straight line but follows the curved surface of 
the sphere to the far ear. For signals of low-frequency, the delay is about $50 \%$ greater because of the phase distortions that result from the reflection and diffraction of sound induced by the sphere, or on real heads, the complex geometry of the head and body. In order to illustrate the relationship between interaural time differences and azimuth, the functions described by Equations 1 and 2 are shown in Figure 1. On real heads, interaural time differences are somewhat greater for angles of incidence greater than $60^{\circ}(.19$ times greater at $75^{\circ}$, and 0.44 times greater at $\left.90^{\circ}\right)(\mathrm{Kuhn}, 1977)$.

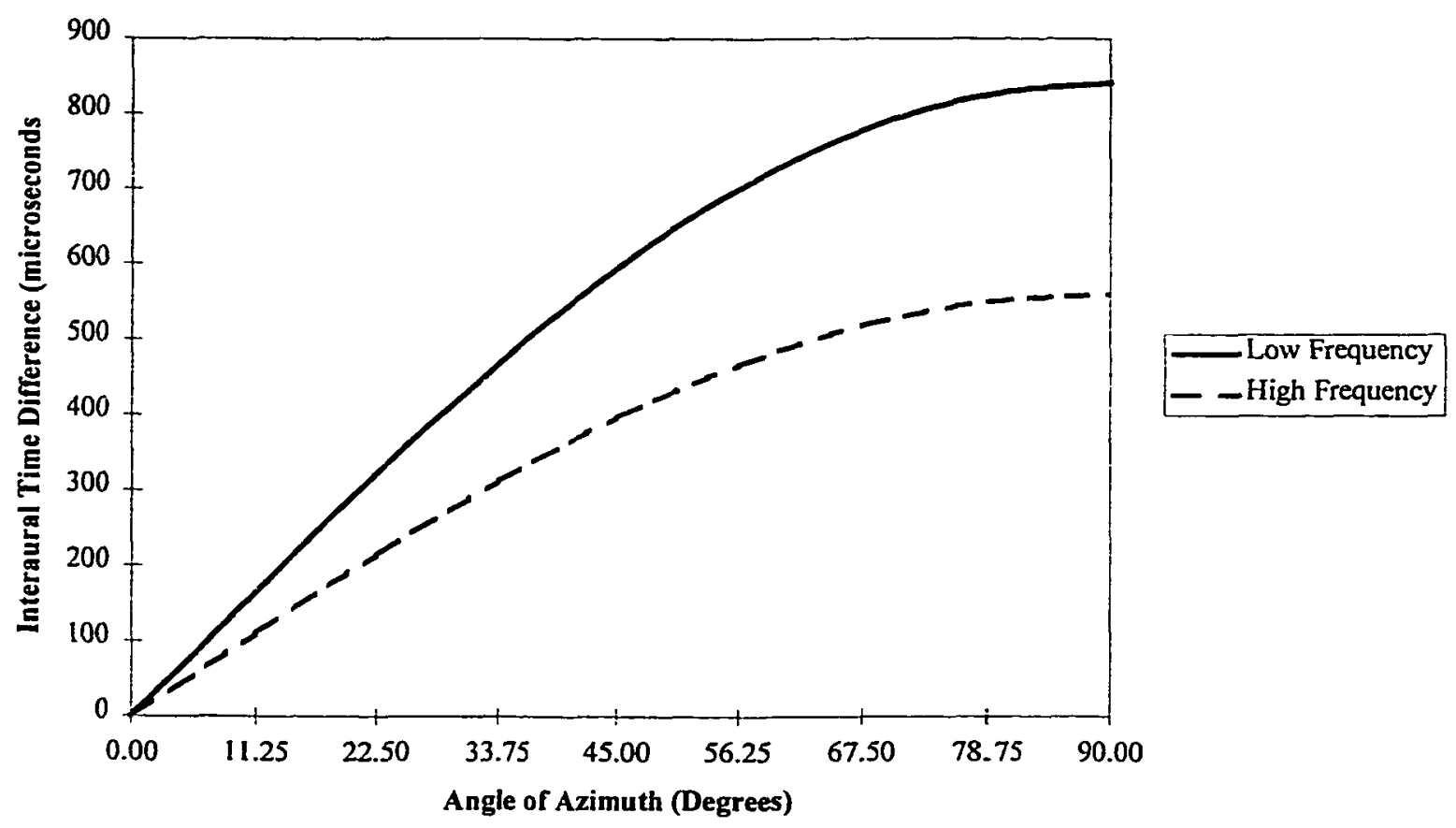

Figure 1: Theoretical values of interaural time difference for horizontal azimuths of interest, based on Kuhn (1977). The solid line represents low-frequency signals ( $>$ $500 \mathrm{~Hz}$ ) while the dashed line represents high-frequency signals $(>2000 \mathrm{~Hz})$.

Head-related transfer functions were measured by Wightman and Kistler (1989) on both ears of ten young adults at six elevations $\left(-36^{\circ},-18^{\circ}, 0^{\circ},+18^{\circ},+36^{\circ}\right.$ and $\left.+54^{\circ}\right)$ and 24 azimuths ( $15^{\circ}$ steps in a complete circle). The interaural time differences extracted 
from these measurements were consistent with the predictions of Kuhn for azimuths around $45^{\circ}$, but for azimuths around $90^{\circ}$ the interaural time differences were considerably larger than predicted (by about $260 \mu \mathrm{s}$ ).

Middlebrooks and Green (1990) measured interaural delays in the envelopes of high-frequency carrier signals for six human subjects as a function of the location of a sound source. The stimulus was a high-pass filtered wide-band noise $(3.0 \mathrm{kHz}$ to 16 $\mathrm{kHz}$ ). In examining the interaural envelope delays computed within a narrow bandwidth that approximated a critical band they found that, to a first approximation, the directional dependence of envelope delays of high-frequency noises up to $16 \mathrm{kHz}$ matches that predicted by modeling the head as a rigid sphere. The high-frequency envelope delays were thus similar to those measured for pure tones by Kuhn (1977). However, the fine structure of the data showed frequency-dependent deviations from predicted values. Similar frequency-dependent deviations in group delay had been observed by Blauert (1983). The deviations in envelope delay reported by Middlebrooks and Green (1990) were related to the bandwidth of the filter used to compute the delay: the greater the bandwidth, the less variation in interaural envelope delays. For bandpass filters comparable in width to a critical band, frequency-dependent variations in interaural envelope delays were on the order of $100 \mu \mathrm{s}$.

\subsection{Interaural intensity differences}

The interaural intensity difference is defined as:

$$
I I D(d B)=20 \log \left(p_{L} / p_{R}\right)
$$

Equation 3

where $I I D(d B)$ is the interaural intensity difference in $\mathrm{dB}$ and $p_{L}$ and $p_{R}$ are the pressure magnitudes at the left and right ears. (Kuhn, 1987). Interaural intensity differences are a 
result of the fact that the human ear is directional in nature, i.e., the sound pressure level that a sound source induces in the ear canal is partly determined by the direction of the sound source relative to the head and torso. In contrast to interaural time differences, interaural intensity differences are highly frequency-dependent because of the differential effect that the head and body have on low-versus high-frequency sounds. Low-frequency sounds have long wavelengths and bend around the head, while high-frequency sounds are shadowed by the head. Besides head shadow, other mechanisms that affect interaural intensity differences are backscattering of sound to the ears from the shoulders or torso and the directivity produced by the pinna.

Shaw (1974) published a summary of data from 12 different studies involving measurements of pressure transformation, azimuthal dependence, interaural intensity difference and ear canal pressure distribution on 100 subjects. He developed a family of curves that best fit the experimental data. Later Shaw and Vaillancourt (1985) published this same family of curves in numerical form. Applying Equation 3 to these data yields a set of theoretical interaural intensity differences for sounds emanating from horizontal angles of azimuth from $0^{\circ}$ to $\pm 90^{\circ}$. These curves are shown in Figure 2, where the dependence of interaural intensity difference on frequency can be clearly seen. 


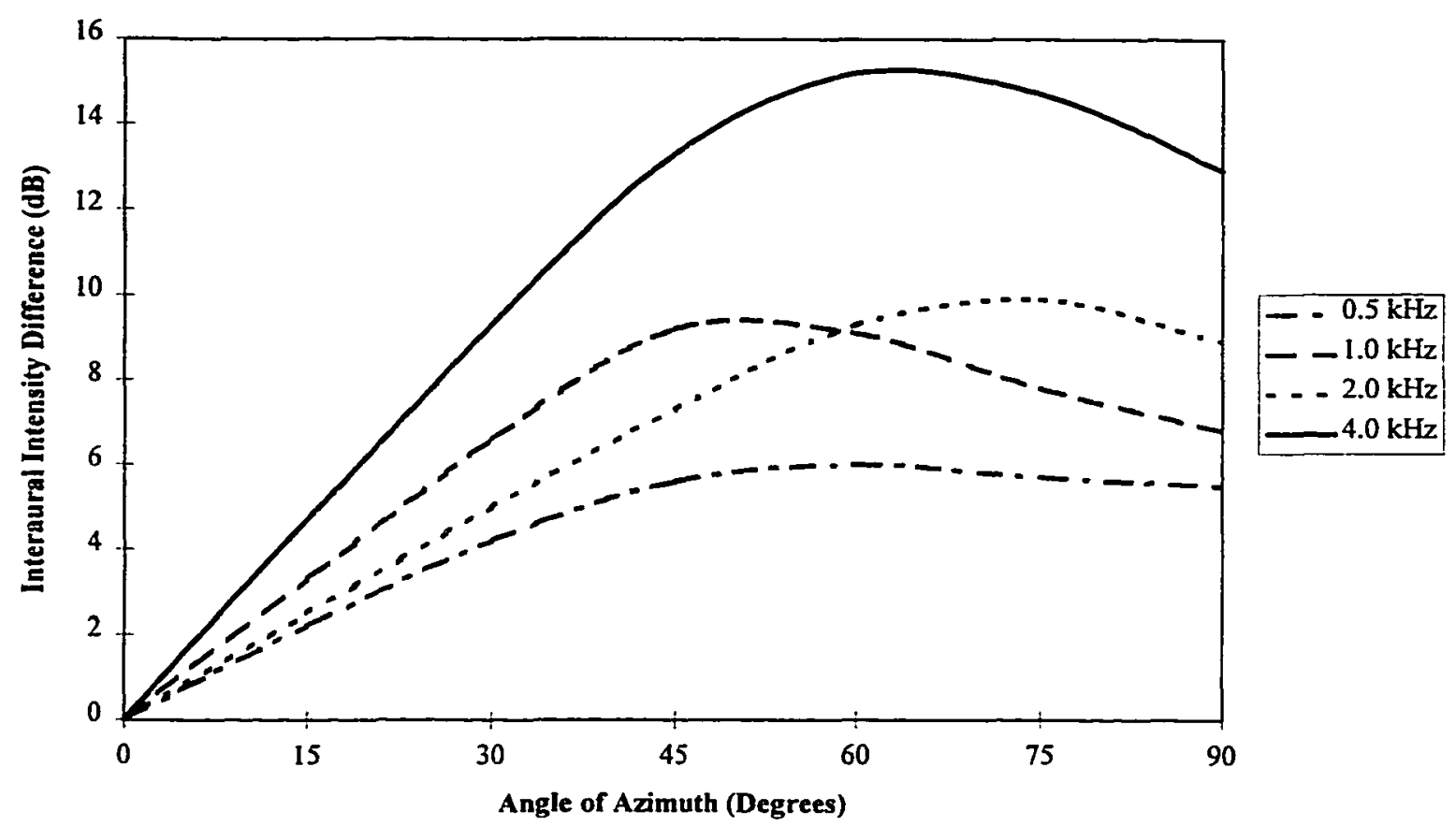

Figure 2: Theoretical interaural intensity differences for azimuths from $0^{\circ}$ to $90^{\circ}$ for frequencies $0.5,1.0,2.0$ and $4.0 \mathrm{kHz}$ (based on Shaw and Vaillancourt, 1985)

It should be noted that Figure 2 represents a smooth idealization of IID versus Frequency. Measurements of interaural intensity differences on real or mannequin heads show a fine structure that varies considerably (Blauert, 1983; Kuhn, 1987; Middlebrooks, et al., 1989). There are also considerable between-subject differences in the pattern of interaural intensity differences over frequency, particularly in the high-frequencies where pinna effects are evident.

\subsection{Relationship Between Interaural Time Differences and Interaural Intensity Differences}

Interaural intensity differences and interaural time differences are not independent phenomena in natural signals. It appears reasonable to assume that the human perceptual system has evolved based upon sound field conditions that occur in everyday life, and that any given individual with hearing in both ears has a set of expectations for interaural 
temporal and intensive relationships that are related to sound source position. A recent study by Gaik (1993) investigated the physical and psychophysical relationships between interaural time differences and interaural intensity differences. Outer ear impulse responses in a free field from 3 subjects for 122 directions in the upper hemisphere were analyzed to measure the interaural intensity difference, interaural phase delay and interaural group delay that occur in each critical band from $20 \mathrm{~Hz}$ to $15.5 \mathrm{kHz}$. Not surprisingly, clear patterns of relationship between interaural intensity differences and interaural phase delay differences, and between interaural intensity differences and interaural group delay differences for each critical band were observed. Thus, for a given measured interaural intensity difference within a critical band, an interaural phase delay or interaural group delay could be predicted with high accuracy. Later psychoacoustic testing using signals with "natural" and "unnatural" combinations of interaural parameters demonstrated that listeners respond differently to signals with unnatural combinations of interaural intensity difference and interaural time difference. (see Section 2.10)

\subsection{The Roles of Interaural Intensity Difference and Interaural Time Difference Cues in Horizontal Localization}

\subsubsection{The Duplex Theory}

The duplex theory of Lord Rayleigh (Strutt, 1907) proposed that horizontal localization was mediated at low-frequencies (up to $770 \mathrm{~Hz}$ ) by interaural phase differences and in the high-frequencies (above $3000 \mathrm{~Hz}$ ) by interaural intensity differences. A classic experiment by Stevens and Newman (1936) appeared to confirm the duplex theory. The pure-tone localization errors made by their listeners were quite small in regions up to about $1000 \mathrm{~Hz}$, became greater in a region around $3000 \mathrm{~Hz}$, and 
then began to decrease again at about 4000 or $5000 \mathrm{~Hz}$. The authors noted that broadband clicks and noises were localized far more accurately than pure tones. This was attributed to the fact that the low-frequency time cues and high-frequency intensity cues support each other, resulting in a more accurate judgment on the part of the observer.

The results of Sandel et al. (1955) supported the duplex theory by demonstrating that localization of tones measured using an acoustic pointer was frequency dependent, with a breakdown of localization accuracy above $1500 \mathrm{~Hz}$. Mills (1958) found that interaural phase differences could not be discriminated at frequencies above $1400 \mathrm{~Hz}$, also lending support to the duplex theory.

The work of Klumpp and Eady (1956) indicated, however, that listeners could detect interaural delays in the envelopes of complex high-frequency signals. In their experiment, listeners were unable to detect interaural time differences for tones of 1500 $\mathrm{Hz}$ and higher. Yet the same subjects could detect interaural time differences in narrow bands of noise which had no components below $3000 \mathrm{~Hz}$. Yost, et al. (1971) showed that the difference limen for interaural time differences in high-pass filtered clicks decreased (i.e., discrimination improved) with the number of slow ( 50 per second) repetitions of the clicks. These data also indicated that interaural temporal cues in the signal envelope of high pass signals can be detected by listeners. Hafter and DeMaio (1975) showed that subjects could lateralize clicks that contained only high-frequency information (higher than $3.0 \mathrm{kHz}$ ), but it was suggested that such cues are less important than low-frequency lateralization cues since their subjects performed less well with high-frequency clicks than with low-frequency clicks. However, Henning (1974a, 1974b) used amplitude modulated tones to demonstrate that subjects could lateralize high-frequency amplitude 
modulated signals ( $3900 \mathrm{~Hz}$ carrier, $300 \mathrm{~Hz}$ modulation) based upon envelope delays as well as they could lateralize a $300 \mathrm{~Hz}$ pure tone.

\subsection{Relative Importance of Interaural Time Difference and Interaural Intensity Difference Cues for Localization}

The detection and lateralization studies cited above indicate that subjects are able to detect and respond to both interaural time and interaural intensity differences in complex sounds. An important practical issue is the relative perceptual importance of interaural time difference versus interaural intensity difference cues in the localization of complex signals with differing frequency content. This question has stimulated a number of conflicting-cue experiments in which interaural time difference and interaural intensity difference cues are manipulated in order to assess their relative importance for the localization of sounds.

There has been much recent interest in simulating free-field spatial listening over headphones (Wightman and Kistler 1989a, 1989b; Loomis, et al., 1990; Gilkey and Anderson, 1997). A benefit of such work is that headphone presentation allows precise control over the various binaural cues that are available for localization while still presenting stimuli that are very close to those that occur in a sound field. For example, one can manipulate interaural time difference cues while leaving other localization cues intact in order to assess their relative importance to the localization of complex signals. Wightman and Kistler (1992) manipulated the interaural phase relations of their simulated free-field signals so that interaural time difference cues signaled one direction while interaural intensity difference and pinna cues signaled another. Specifically, subjects localized broad-band stimuli that fell into one of the three following categories: 
- The interaural time difference was zero, but the interaural intensity difference and spectral shape was normal.

- The interaural time difference was equivalent to that at $-45^{\circ}$ azimuth, but the interaural intensity difference and spectral shape was normal.

- The interaural time difference was equivalent to that at $90^{\circ}$ azimuth, but the interaural intensity difference and spectral shape was normal.

In each of these conditions, interaural time difference cues dominated the localization judgments of the listeners as long as sufficient low-frequency energy was present in the signal. When the low-frequency content $(<\sim 1500 \mathrm{~Hz})$ of the signal was removed by high pass filtering, localization was accurate and not affected by the adjustments of interaural time difference. As lower and lower frequency information was added to the stimuli, subjects' responses were increasingly more affected by the interaural time difference cues. This finding is consistent with the duplex theory since for these signals and conditions, interaural intensity cues dominated the localization of the signals that contained no low-frequency components, and interaural time difference cues dominated in signals that contained substantial low-frequency energy.

Another conflicting-cue experiment performed by Wightman and his colleagues is described in a recent book chapter (Wightman and Kistler, 1997). In this experiment the interaural intensity differences in wide-band virtual sound sources were adjusted to point to $0^{\circ}$ azimuth, and the interaural time differences left intact. No bias in the horizontal localization judgments toward the median plane was observed, as might be expected if the interaural intensity difference cues were contributing to the localization judgments of the listeners. The principal effect of interaural intensity difference manipulation was to 
increase the number of front/back confusions and to disrupt judgments of elevation. Once again interaural time difference cues appeared to dominate the localization decisions of the listeners.

Wightman (1997) explains these findings based upon a hypothesis that cue reliability (or consistency) determines the weight applied by the perceptual system to a given localization cue. According to this hypothesis, interaural time difference cues do not depend upon source characteristics (what the source "sounds like") and are not highly frequency-specific or idiosyncratic, therefore they rate high in the reliability scale. On the other hand, interaural time difference cues also reflect a certain degree of ambiguity because a given interaural time difference indicates a range of possible source positions, i.e., "cones of confusion" (Woodworth, 1938). Interaural intensity differences, however are also reliable cues in that they do not depend upon source characteristics, but unlike interaural time difference cues, they are highly frequency-specific and idiosyncratic. Thus interaural intensity difference cues may rate lower on the scale of reliability than interaural time difference cues. The data from the Wightman (1997) experiment indicate that interaural time difference cues dominated judgments of horizontal localization, but that interaural intensity difference cues were important for resolving front/back ambiguities and for vertical localization judgments. Interaural time difference cues are reliable predictors of horizontal azimuth, but are ambiguous with regard to elevation and front/back discrimination. In contrast, interaural intensity differences are not ambiguous - with regard to elevation and front/back discrimination. Thus, the perceptual system must have relied more heavily on interaural intensity difference cues for these judgments. 


\subsection{Auditory Localization In Rooms}

The localization of sound sources in rooms is an area that until recently has not received a great deal of research attention, presumably because of the difficulty of controlling room acoustics. It has seemed apparent for years that the precedence effect (Wallach, et al., 1949; Haas, 1951; Blauert, 1971; Zurek, 1980) plays a significant role in localization in a reverberant environment. That is, the first-arriving wave front will largely determine the directionality of a sound, while later-arriving reflections from walls, ceiling, floor and objects in the room will carry significantly less perceptual weight.

A series of experiments conducted by Hartmann, Rakerd and their colleagues focused upon the effect that room reflections have on auditory localization. The first experiment of this series (Hartmann, 1983) used a variable-acoustics concert hall (the Espace de Projection, or ESPRO, in Paris) to change the acoustical properties of the listening environment as subjects identified the source locations of tones and noises with varying onset characteristics. One important finding was that the localization of impulsive sounds in a room may depend upon room geometry. Specifically, an improvement in localization accuracy was related to the lowering of the ceiling and a concomitant increase in ceiling reflections. Ceiling reflections, on average, tend to agree with the azimuth of the sound source and may have been reinforcing the listeners' sense of location. A later experiment confirmed that the precedence effect does not eliminate all influences of room reflections (Rakerd and Hartmann, 1985). In this study, a single reflecting surface was used to simulate a floor, ceiling, left and right wall in an otherwise anechoic chamber. The presence of a reflecting surface on the side tended to degrade the accuracy of localization of $50 \mathrm{~ms}$ tone bursts of $500 \mathrm{~Hz}$, turned on and off at zero 
crossings, while the presence of a reflecting surface on the "floor" or "ceiling" neither degraded nor enhanced localization accuracy. Interestingly, no systematic bias in the direction of the interfering reflection resulted, but only a reduction of accuracy. When the experiment was repeated with a very slow onset ( 7 seconds) continuous $500 \mathrm{~Hz}$ tone in order to eliminate the effect of the rapid onset of the tone, localization performance degraded a great deal and systematic biases in the data were observed. When a floor or ceiling was present, the bias was toward the center and when a right or left wall was present the biases were more complex. Interestingly, the right and left wall conditions were mirror images of each other indicating that subjects used similar strategies in these two conditions. In trying to account for these results, the investigators measured the interaural time differences and interaural intensity differences of a mannequin head placed near the positions that the subjects' heads had occupied during the experiment. They found that strong influences on both interaural time difference and interaural intensity difference occurred as a result of the interference of room reflections. Interaural intensity differences dominated the subjects' localization judgments for the steady-state $500 \mathrm{~Hz}$ tone in the ceiling, left- and right-wall conditions. In these conditions, interaural time difference values were highly elevated as a result of room reflections. By comparing the localization judgments of the subjects with the measured interaural time differences and interaural intensity differences for the various speaker locations, it seemed that subjects were ignoring interaural time differences and basing their judgments on interaural intensity differences. This result indicates not only that interaural intensity differences are affected by the characteristics of a room, but also that they can have a 
substantial effect on localization, even at low-frequencies when the signal contains no transient information.

\subsection{Plausibility/Reliability of Acoustic Cues for Localization}

In interpreting the data from these investigations, Rakerd and Hartmann (1985) formed what they called the plausibility hypothesis. According to this hypothesis, interaural time difference cues are weighted by subjects according to their plausibility. Subjects in these experiments were able to see the loudspeaker locations and could assess the plausibility of the interaural time differences that they were hearing. Because the interaural time differences were out of the range of those that would be expected in a free field, it was hypothesized that they were ignored by the listeners, and judgments of location were based more heavily upon interaural intensity differences. However, the plausibility hypothesis as it is formulated by Hartmann and his colleagues regards only interaural time differences as subject to plausibility assessment, and not interaural intensity differences. In the Rakerd and Hartmann (1985) study, an interaural intensity difference of $10 \mathrm{~dB}$ was measured for a $500 \mathrm{~Hz}$ tone generated at one of the loudspeaker locations. Interaural intensity differences for low-frequency tones are unlikely to be greater than $\sim 6 \mathrm{~dB}$ at the most extreme azimuths (Shaw and Vaillancourt, 1985) in a freefield situation. The argument was that this rather implausible interaural intensity difference appeared to form the basis for the subjects' localization judgment. In a later test of the plausibility hypothesis, a conflicting-cue experiment (Hartmann and Fontana, 1991; Hartmann, 1997) was conducted. Signals (500 Hz tone pulses and $500 \mathrm{~Hz}$ tones without onset transients) that had been recorded in an anechoic room from 12 speakers separated by $3^{\circ}$ of azimuth were presented via headphones to listeners sitting in the same 
location in which the recording was made. The recorded signals were edited to produce variable interaural intensity difference cues from $0 \mathrm{~dB}$ to $25 \mathrm{~dB}$, but interaural time difference cues remained unaltered. The results showed that interaural time difference cues dominated the localization judgments of all subjects for artificial interaural intensity differences less than $5 \mathrm{~dB}$, and some subjects were unaffected in their judgments by interaural intensity differences of 10 or $15 \mathrm{~dB}$. However, grossly implausible interaural intensity differences (15 to $25 \mathrm{~dB}$ ) strongly influenced localization judgments for all of the subjects.

In a study by Gaik (1993) that investigated the relationships between interaural time differences and interaural intensity differences in a free field (see Section 2.6), the reactions of listeners to interaural differences that do not conform to those expected under natural listening situations were investigated. The signals used in the experiment were two narrow bands of noise $(400 \mathrm{~Hz}-510 \mathrm{~Hz}$ and $3700 \mathrm{~Hz}-4350 \mathrm{~Hz})$ presented dichotically with combinations of five interaural time differences $(0, \pm 300$ and $\pm 600 \mu \mathrm{s})$ and five interaural intensity differences $(0, \pm 6$ and $\pm 12 \mathrm{~dB})$. Ten normally hearing subjects were asked to describe the perceived lateral position of the signals and to rate the naturalness of the sound on a scale from 0 (natural) to 10 (unnatural). The more signals deviated from natural combinations of interaural parameters, the more likely it was that listeners would rate them unnatural. Furthermore, the variance of the subjects' lateralization judgments increased significantly for combinations of interaural intensity and time difference that did not occur naturally based upon the results of the measurements made in the first part of the study. In addition there were far fewer split images for the natural combinations of interaural time difference and interaural intensity 
difference than for unnatural combinations. These results are consistent with the hypothesis that the auditory perceptual system takes into account the plausibility of interaural cues when making spatial judgments. Under the lateralization conditions of the Gaik (1993) experiment, however, subjects did not seem to assess the plausibility of interaural time difference cues differently from interaural intensity difference cues, as suggested by Hartmann (Rakerd and Hartmann, 1985; Hartmann, 1997) in his plausibility hypothesis.

Wenzel et al. (1993) conducted a study in which listeners made localization judgments for virtual wide band noise bursts that were generated using the head-related transfer function of another individual, i.e., they "listened through the ears of a different person." Localization performance in the horizontal plane was not different from that when listening to the same sounds broadcast through loudspeakers in the free field, but there was a greater number of front/back confusions as well as confusions between high and low elevations. Wightman (1997) suggests that these results are evidence that listeners weight localization cues based upon their plausibility. While the interaural time difference and interaural intensity difference cues that listeners heard through the ears of another individual were plausible and reliable, the spectral localization cues that they heard were unfamiliar and therefore given less weight.

\subsection{Research Questions and Hypotheses}

According to Wightman and Kistler (1997), the reliability of an auditory - localization cue is determined by the extent to which it

- depends upon source characteristics (requires that the listener remember what the acoustic event "sounds like"), 
- provides the same information across bands of frequency,

- is the same across listeners and

- is unambiguous.

Interaural time difference rates as the most reliable cue according to this scheme. Although it is ambiguous (the same interaural time difference can be found at many different source locations in the lateral plane), interaural time difference does not depend upon source characteristics and is similar across frequency bands and across listeners. Like interaural time difference cues, interaural intensity difference cues do not depend upon source characteristics, but they do differ across frequency bands and are idiosyncratic, particularly in the high-frequencies. In addition, interaural intensity difference cues are subject to ambiguity, even within $\pm 90^{\circ}$ of azimuth. Thus interaural intensity difference cues may be considered less reliable than interaural time difference cues.

The theory upon which Wightman and Kistler (1997) base their discussion appears to be that a reliable localization cue will dominate the determination of apparent source position over a less reliable cue. For situations in which cues conflict, the more reliable cue will determine the outcome of the localization judgment. This argument formed the basis of the authors' explanation of the results of two conflicting cue experiments (Wightman and Kistler, 1992; Wightman and Kistler, 1997). In these experiments, described above, either interaural time difference or interaural intensity difference was manipulated in the localization of broad band stimuli. In either case, as long as sufficient low-frequency information was available in the signal, interaural time difference cues dominated in the front horizontal plane when they conflicted with other 
localization cues. Each of these experiments used wide-band signals in which an abundance of low-frequency energy was available ( $250 \mathrm{~ms}$ burst of Gaussian noise with a nominally flat spectrum from 0.2 to $14 \mathrm{kHz}$ ) in at least some conditions.

A localization experiment by Hartmann and Fontana (1991) and reported by Hartmann (1997), also described above, used $0.5 \mathrm{kHz}$ tone pulses as well as steady-state $0.5 \mathrm{kHz}$ tones with conflicting interaural time difference and interaural intensity difference cues. While Wightman and Kistler (1997) eliminated interaural intensity difference cues, Hartmann and Fontana (1991) amplified them by 5 to $25 \mathrm{~dB}$ (in $5 \mathrm{~dB}$ steps). When the amplification of interaural intensity differences exceeded 10 to $15 \mathrm{~dB}$, significant horizontal localization bias was introduced. Plausible and natural interaural time difference cues were not sufficient to override the effect of large interaural intensity difference manipulations, even for these low-frequency signals.

It would seem from these conflicting results that an explanation based upon the theory that the most reliable cue (i.e., interaural time difference in the horizontal plane) dominates auditory localization performance is unsatisfactory. The $500 \mathrm{~Hz}$ tones of Hartmann and Fontana (1991) carried sufficiently salient interaural time difference cues. If such cues are the most reliable and therefore dominate localization judgment, one would have expected the localization of these signals to be unaffected in the front medial plane by manipulations of interaural intensity difference cues.

\subsubsection{Statement of Theory and Experimental Hypotheses}

An alternative theory is proposed in the present study. While cue reliability and plausibility are factors that no doubt affect auditory localization judgments, the dominance of the most reliable cue present is not absolute. Despite the relatively better reliability of interaural time difference cues, listeners nevertheless take advantage of 
interaural intensity difference cues when performing auditory localization tasks in the front medial plane. It is expected that adjustments of interaural intensity cues such as those that occur with binaural amplitude compression hearing aids will affect horizontal auditory localization judgments. The effect of interaural intensity difference adjustments will be particularly strong when the attenuation is substantial in magnitude (greater than about $10 \mathrm{~dB}$, based upon the results of Hartmann and Fontana, 1991) or where interaural time difference cues are less salient (as in the case of signals with little or no lowfrequency energy). That is, the effect of adjusting the interaural intensity differences will be frequency-dependent as well as dependent upon both the magnitude of the naturally occurring interaural intensity difference and the extent of the adjustment. Thus, the first three experimental hypotheses are proposed for this experiment:

- Experimental Hypothesis \#1 - Subjects who attempt to identify the horizontal location of the source of sounds in which interaural intensity differences have been attenuated by $50 \%$ or $100 \%$ of their original $\mathrm{dB}$ level will make errors that reflect a bias toward the center.

- Experimental Hypothesis \#2 - For third-octave band stimuli in which interaural intensity differences have been attenuated by $50 \%$ or $100 \%$ of their original $\mathrm{dB}$ level, the magnitude of localization errors will increase with increasing center frequency.

- Experimental Hypothesis \#3 - For band-pass stimuli in which interaural intensity differences have been attenuated by $50 \%$ or $100 \%$ of their original $\mathrm{dB}$ level, the magnitude of localization errors will increase as the lowfrequency energy content decreases. 
The results of Gaik (1993) indicate that the variability of subjects' localization judgments is greater for signals with unnatural combinations of interaural time and intensity differences than for natural signals. Unnatural combinations of interaural time differences and interaural intensity differences will be expected for many situations in which interaural intensity differences are attenuated. The attenuation of interaural intensity differences by $50 \%$ and $100 \%$ will therefore be likely to result in less precise localization judgments (i.e., greater variability) than that expected for signals with natural combinations of interaural time and intensity differences. The fourth experimental hypothesis addresses this expectation.

- Experimental Hypothesis \#4 - The variability of subjects' localization judgments will increase when interaural intensity difference cues are attenuated by $50 \%$ or $100 \%$ of their original $\mathrm{dB}$ value.

Rakerd and Hartmann (1985), found that significantly large interaural time and intensity differences may occur as a result of interference between reflections and direct sound in a room with reverberant walls. Thus, listener's judgments of sound source location, which are largely mediated by interaural time and intensity cues, may be affected by the acoustics of the room. Unnatural combinations of interaural time and intensity cues may result in greater response variability (Gaik, 1993) under conditions of reverberation, assuming that the signals to be localized have no significant impulsive components that may activate the precedence effect (Wallach, et al., 1949). The fifth experimental hypothesis addresses this possibility. 
- Experimental Hypothesis \#5 - The localization of signals recorded in a reverberant environment will be more variable than for those recorded in an anechoic chamber.

Signals recorded in a reverberant environment may already have substantial variability in interaural intensity difference relative to a non-reverberant environment because of interference between the direct sound and its reflection. As indicated above, this observation was made by Rakerd and Hartmann (1985) for slow-onset $0.5 \mathrm{kHz}$ tones. Under these conditions, auditory localization may be somewhat degraded in a reverberant room, and further manipulation of interaural intensity differences may have less of an impact than would manipulation of these cues in a free field. If this is true, then localization of signals that were recorded in a reverberant room may be less affected by attenuated interaural intensity differences than signals recorded in an anechoic chamber. The sixth experimental hypothesis addresses this possibility.

- Experimental Hypothesis \#6 - The localization of signals recorded in a reverberant environment will be less affected by the attenuation of interaural intensity differences than those recorded in an anechoic chamber. 


\section{CHAPTER 3: METHOD}

\subsection{Stimuli}

Stimuli consisted of four third-octave bands of noise and three band-pass noises with varying amounts of low-frequency content.

The nominal frequency limits ( $6 \mathrm{~dB}$ points) of each signal are presented in Table 1 , and the spectra of the signals are shown in the Appendix in Figure 42 through Figure 48.

\section{Table 1: The frequency limits of the experimental signals.}

\begin{tabular}{|l|r|r|}
\hline \multicolumn{1}{|c|}{ Noise Band } & Lower Limit (Hz) & Upper Limit (Hz) \\
\hline $0.5 \mathrm{kHz}$ Third-octave Band & 440 & 550 \\
\hline $1.0 \mathrm{kHz}$ Third-octave Band & 890 & 1120 \\
\hline $2.0 \mathrm{kHz}$ Third-octave Band & 1788 & 2225 \\
\hline $4.0 \mathrm{kHz}$ Third-octave Band & 3525 & 4400 \\
\hline $0.5 \mathrm{kHz}$ to $4.0 \mathrm{kHz}$ Band-pass & 450 & 4275 \\
\hline $1.0 \mathrm{kHz}$ to $4.0 \mathrm{kHz}$ Band-pass & 900 & 4275 \\
\hline $2.0 \mathrm{kHz}$ to $4.0 \mathrm{kHz}$ Band-pass & 1800 & 4275 \\
\hline
\end{tabular}

\subsubsection{Construction of the Experimental Signal Sources}

Figure 3 shows how the experimental signal sources were constructed. The signals were generated by the white noise generator of a Grason-Stadler GSI-16 audiometer. Signals were filtered by a General Radio 1925 One Third-octave Band Multifilter and recorded on digital audio tape (DAT) using a Panasonic Portable Digital Audio Tape Recorder (SU-255). The signals were played back and re-filtered by cascading the signals through both channels of an Ithaco 4302 Dual $24 \mathrm{~dB} /$ Octave Filter that was set to band-pass the signals at their nominal frequency limits. This was done in order to reject as much of the energy lying outside the nominal limits of the desired noise bands as possible. Filtered signals were amplified (NAD Stereo Pre-amplifier 1020A) and digitized on a Data Translation signal processing board (DT2828) at a sampling rate of 24 
$\mathrm{kHz}$ with 12-bit resolution. An IBM compatible personal computer with an $80286 \mathrm{CPU}$ served as the host for the DT2828 signal processing board.

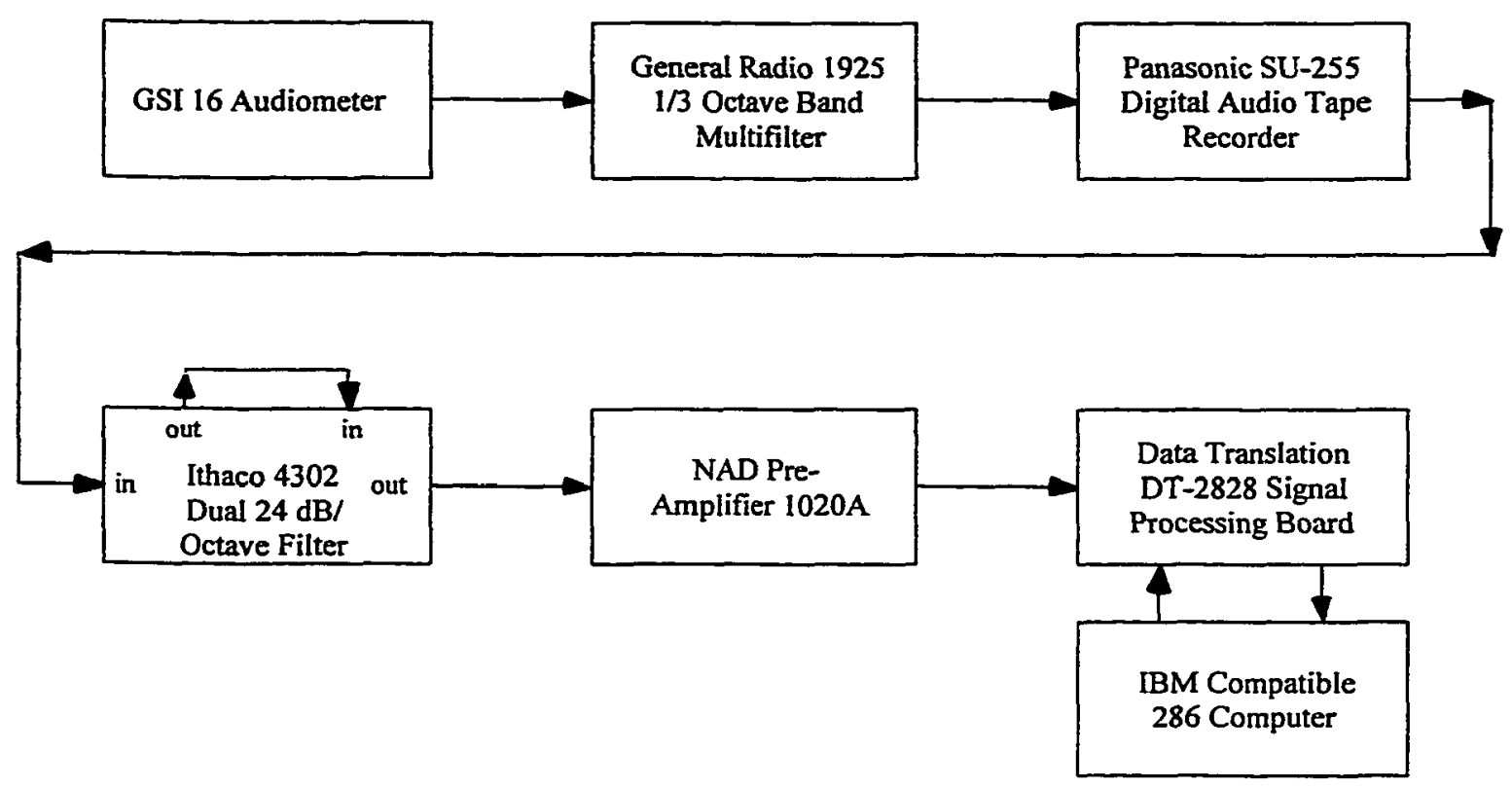

Figure 3: Instrumentation for generating the noise band stimuli.

\subsubsection{Gating of the Experimental Signal Sources}

The duty cycle for the presentation of the experimental stimuli is illustrated in

Figure 4. The source signals were digitally gated into three $340 \mathrm{~ms}$ pulses with $20 \mathrm{~ms}$ raised cosine rise and fall times using custom software. This was done in order to provide multiple repetitions of the signal and sufficient signal on-time for the subjects to establish a localization judgment. The raised cosine rise/fall ramps were intended to reduce spectral splatter that would accompany sudden signal onsets, yet provide some gentle signal onset information during the presentation interval. There were three bursts of noise per stimulus item; each burst was separated from the other by $50 \mathrm{~ms}$ of silence. Each signal presentation had a total duration of $1120 \mathrm{~ms}[340 \mathrm{~ms}+50 \mathrm{~ms}+340 \mathrm{~ms}+50 \mathrm{~ms}+$ 
$340 \mathrm{~ms}$. The gated signals were transferred to digital audio tape (Panasonic Portable Digital Audio Tape Recorder SU-255).

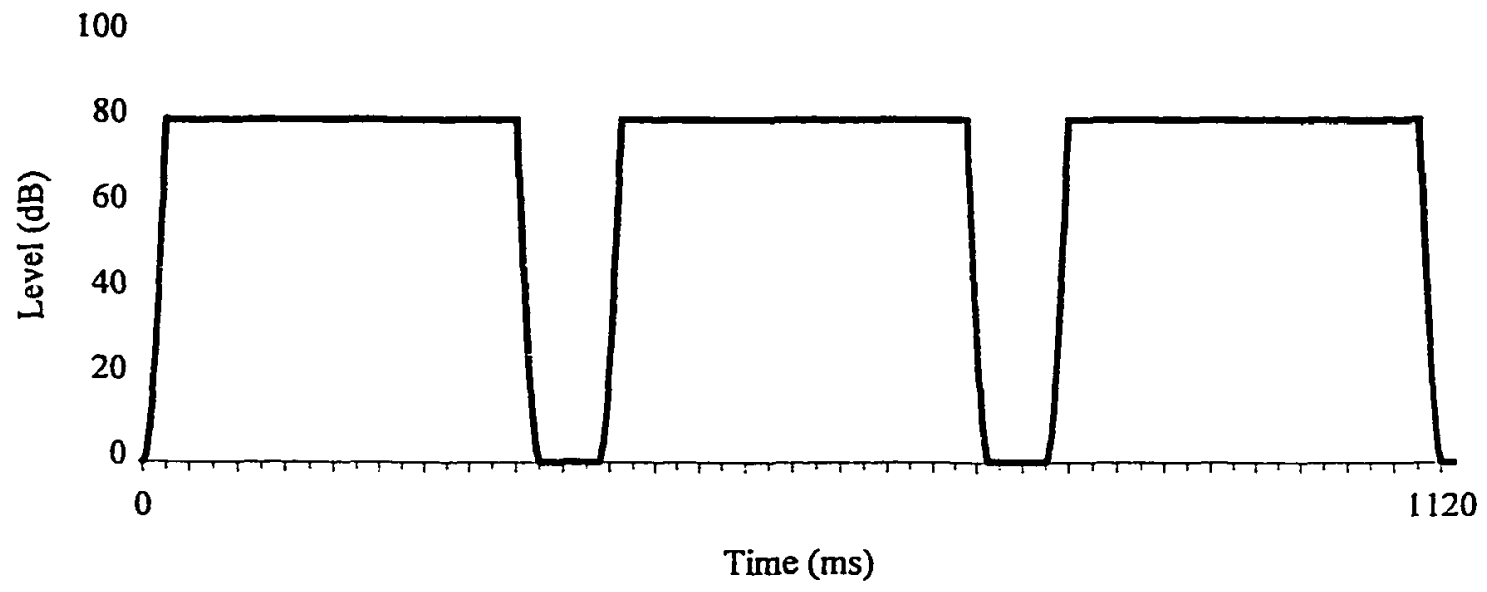

Figure 4: Representation of the test signal duty cycle.

\subsubsection{Recording the Binaural Signals}

A Knowles Electronics Manikin for Acoustic Research (KEMAR) (Burkhard and Sachs, 1975) was placed in each of two rooms, as shown in Figure 5 and Figure 6.

Seventeen loudspeaker locations in a $180^{\circ}$ arc around KEMAR were identified and marked for each room. Each speaker location was separated from the next by $11.25^{\circ}$ and was placed at a distance of 1 meter from KEMAR at ear level. During recording sessions, the signals were played on a DAT player and broadcast by a loudspeaker that was placed at each one of the 17 locations around KEMAR. Signals were presented at a level of 85 $\mathrm{dB}$ SPL, although there was some small variation in level between signals of different frequency $( \pm 2 \mathrm{~dB}$ ). Binaural recordings of each of the signals at each of the loudspeaker locations using the same loudspeaker were made at the "ear drums" of KEMAR. Sixteen 
different tokens of each stimulus type were recorded at each loudspeaker location, however only the first five tokens of each signal type were used for this experiment.

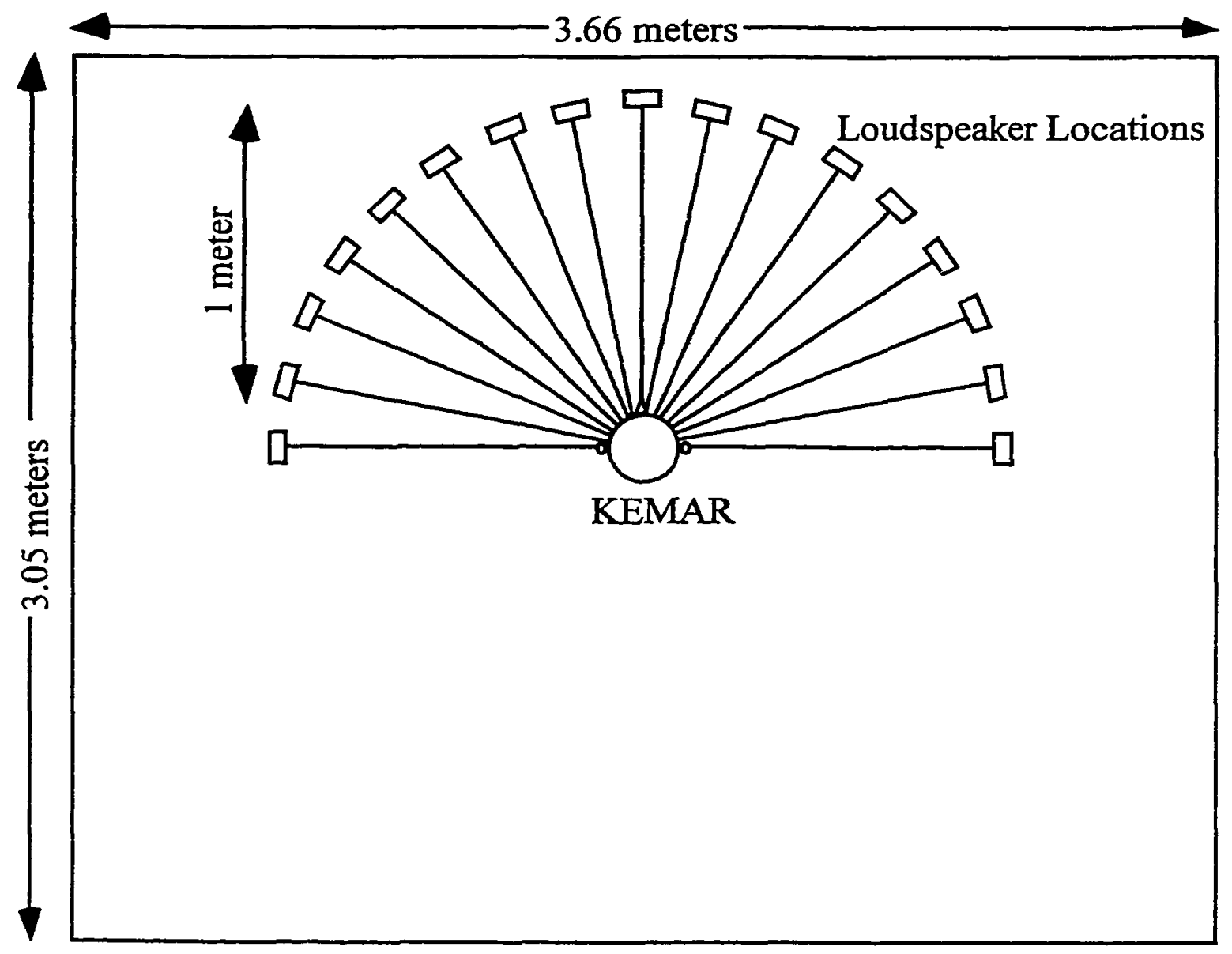

Figure 5: Layout for binaural recordings in the anechoic room. 


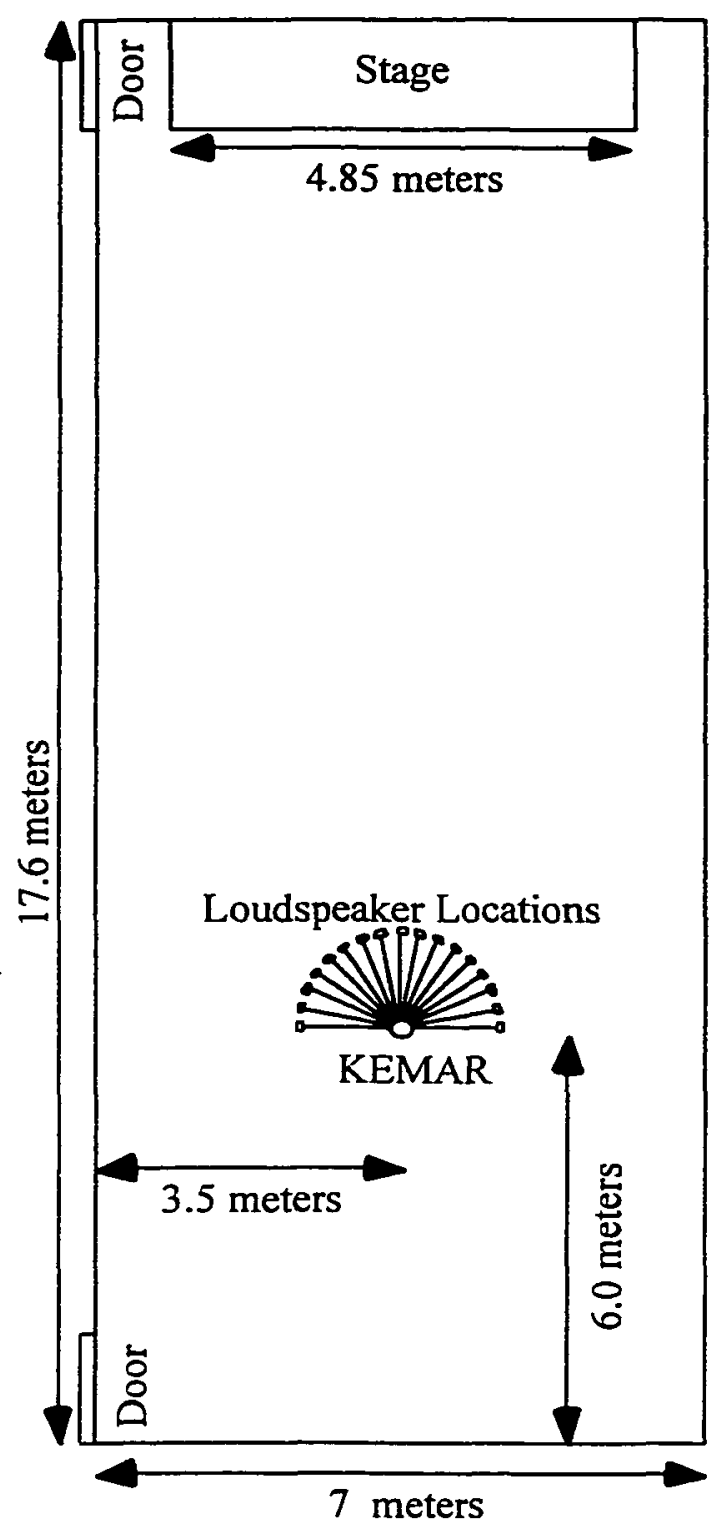

Figure 6: Layout for binaural recordings in the minitheater.

The instrumentation for making the binaural recordings is shown in Figure 7. The source signals were generated by a digital audio tape recorder (Panasonic Portable Digital Audio Tape Recorder SU-255), amplified (Radio Shack SA-150 Integrated Stereo Amplifier Model \#31-1955) and played through a 3-inch loudspeaker (Realistic ${ }^{\circledR}$ Minimus ${ }^{\circledR}-0.3$ Cube Speaker System). KEMAR was fitted with pinnae numbers $150704 \mathrm{R}$ and $150704 \mathrm{~L}$ for the right and left ears, respectively. Zwislocki couplers (DB100 in the 
right ear, DB 4005 in the left ear) were mounted in KEMAR, fitted with B\&K 1/2-inch pressure microphones (4134) and attached to B\&K Adapters (UA0122). The output of each of the two adapters was fed into a B\&K power supply (2801) through a B\&K Preamplifier Cable (AO 0027). The output of each of the power supplies was connected either to the right or left line input receptacle of a second digital audio tape recorder (Panasonic Portable Digital Audio Tape Recorder SU-255).

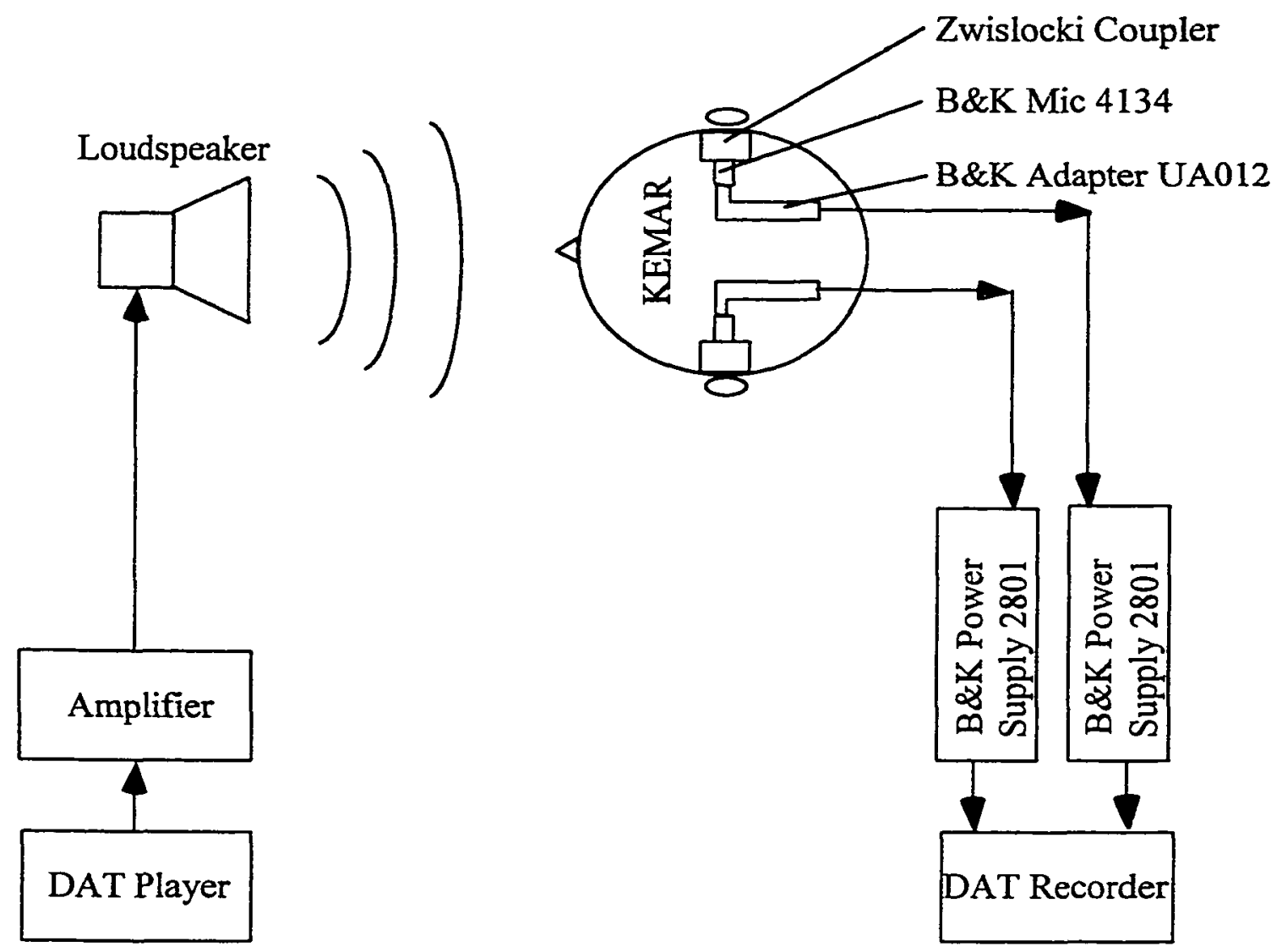

Figure 7: Instrumentation for recording experimental binaural stimuli.

In addition to recording the experimental stimuli, measurements of the directional responses of KEMAR's ears were made using SYSid ( Copyright 1992 Ariel Corporation, Highland Park, NJ) with a chirp stimulus at each speaker location in each room. This was done to provide a database of interaural intensity differences and 
interaural time differences for signals at the different azimuths used in this experiment. It was thought that such a database might prove useful for interpretation of the experimental results. The instrumentation used for making these measurements is shown in the Appendix in Figure 49. For test signal generation, the DAT recorder was replaced by the output circuit of a signal processing board (Ariel DSP-16) under the control of an IBMcompatible 286-based personal computer. For test signal reception, the digital audio tape recorder used for recording the experimental stimuli was replaced by a preamplifier (Rane SM26), an amplifier (Radio Shack SA-150 Integrated Stereo Amplifier Model \#31-1955) and the left and right input channels of the signal processing board (Ariel DSP-16).

Recordings were made in two different rooms: an anechoic chamber and a school assembly room called the minitheater. Both rooms are located in The Lexington School for the Deaf / Center for the Deaf in Jackson Heights, New York. The anechoic room measures 3.66 meters by 3.05 meters with a 2.29 meter high ceiling (total volume was $\left.25.56 \mathrm{~m}^{3}\right)$, and has a double-wall for sound isolation. The reverberation time of the anechoic room was not measured but was presumed to be close to zero. The minitheater room measures 7.0 meters by 17.6 meters, has no windows, is carpeted and has a 2.74 meter high hung ceiling of acoustically absorbent tile (total volume was $337.57 \mathrm{~m}^{3}$ ). There is a 0.41 meter high stage measuring 4.85 meters wide at the far end of the room relative to where the recordings were made. The room has two doors that were closed during recording. The reverberation time (RT-60) of the room was measured using a chirp stimulus (BIGSYSID.EXE, version 4.0, Ariel Corp., 1991). The same instrumentation that was used to measure the directional responses of KEMAR's ears was 
used for the measurement, except that KEMAR was replaced by a microphone stand holding a B\&K $41351 / 2$ " microphone attached to a B\&K preamplifier cable (AO 0027). The same loudspeaker that was used to record the experimental signals was placed at a distance of one meter from the microphone and used to broadcast the measurement stimuli. The reverberation time of the minitheater room was determined to be $400 \mathrm{~ms}$.

In addition, the sound pressure level of the minitheater was measured using a B\&K model 2305 sound level meter and a B\&K model 4135 1/2" microphone. The Aweighted ambient noise level during the recording session was $37 \mathrm{~dB} \mathrm{~L}_{\text {eq }}$.

\subsubsection{Digitizing the Binaural Signals}

The next step in signal preparation involved digitizing the binaural signals. Figure 8 shows the instrumentation used to transfer the signal recordings to computer files. The signals were played on a digital audio tape recorder (Sharp Digital Audio Tape Deck SXD100), and the left and right channels were pre-amplified (Rane SM26), amplified (Yamaha Power Amplifier P2150) and attenuated to ensure that all experimental stimuli were equivalent in level at $0^{\circ}$ azimuth. The signals were then high-pass filtered using an Ithaco 4302 Dual $24 \mathrm{~dB} /$ Octave Filter with a cut-off frequency of $250 \mathrm{~Hz}$. This was done in order to remove a $60 \mathrm{~Hz}$ hum that had been picked up from the instrumentation during the recording. The filtered signals were sent to the left and right channels, respectively, of a digital signal processing board (Data Translation DT-2828) under the control of an IBM compatible 286-based personal computer. The signals were digitized at a 12-bit resolution using a sampling rate of $12 \mathrm{kHz}$ in each channel, with a simultaneous sample and hold strategy to ensure that temporal features were not disrupted. The first five tokens of each signal type were extracted from the resulting computer files and became 
the signal set for use in the experiment. The total number of signal files in this set was 1,190: 5 tokens, 7 signal types, 17 azimuths and 2 rooms.

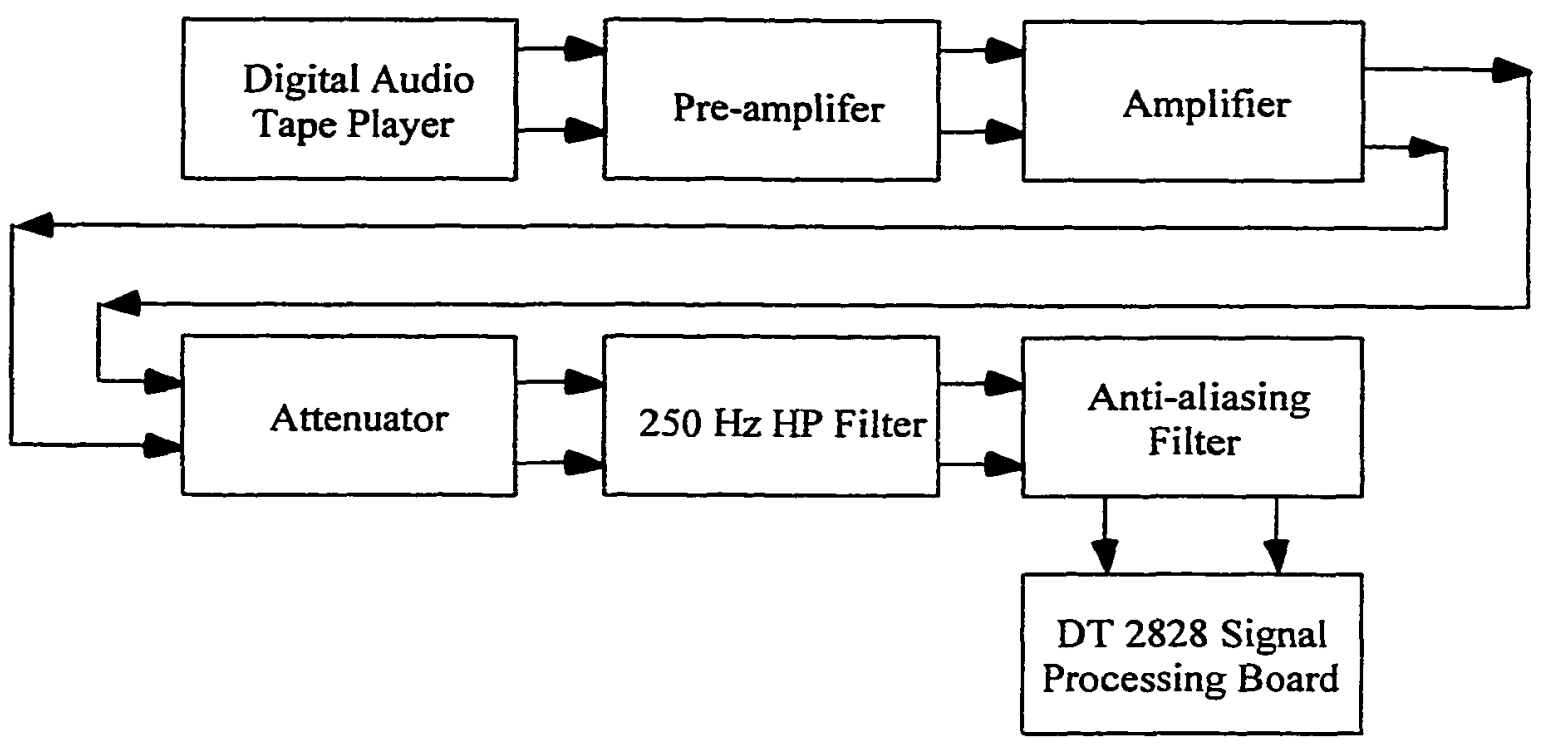

Figure 8: Instrumentation for digitizing the binaural signals.

\subsubsection{Processing the Binaural Signals}

The digital experimental signals were processed in one of two ways: a) the interaural intensity difference was attenuated by $50 \%$ (to one half of its original value in $\mathrm{dB}$ ), or b) the interaural intensity difference was attenuated by $100 \%$ (to $0 \mathrm{~dB}$ ). The interaural time difference was not modified. Interaural intensity difference was defined as the difference in root mean square level between the signal recorded in the right ear of KEMAR and the signal recorded in the left ear of KEMAR. The root mean square level of each channel was measured and the level adjustments necessary to achieve the targeted interaural intensity difference were calculated. When modifying the interaural intensity differences, care was taken to preserve the summed loudness of the binaural signal. The channel with the lower level was amplified and the channel with the greater level was attenuated so that the level of the modified binaural signal had the same summed energy 
in $\mathrm{dB}$ as did the original signal. For signals in which the interaural intensity difference was attenuated by $100 \%$, Equation 4 was used to determine the level in the right and left channels for the processed signals. For signals in which the interaural intensity difference was attenuated by $50 \%$, Equation 5 and Equation 6 were used to determine the levels in the right and left channels, respectively, for the processed signals.

$$
\begin{gathered}
\operatorname{Radj}^{=} \operatorname{Ladj}=10 \times \log \left[\frac{10^{\frac{R}{10}}+10^{\frac{L}{10}}}{2}\right] \\
\operatorname{Radj}=10 \times \log \left[\frac{10^{\frac{R}{10}}+10^{\frac{L}{10}}}{10^{\frac{L-R}{20}}+1}\right] \\
\operatorname{Ladj}=10 \times \log \left[\frac{10^{\frac{R}{10}}+10^{\frac{L}{10}}}{10^{\frac{R-L}{20}}+1}\right]
\end{gathered}
$$

where:

$R=$ original $\mathrm{rms}$ level $(\mathrm{dB})$ in the right channel

$L=$ original $\mathrm{mms}$ level $(\mathrm{dB})$ in the left channel

$R_{a d j}=$ adjusted $\mathrm{rms}$ level $(\mathrm{dB})$ in the right channel

$L_{a d j}=$ adjusted $\mathrm{rms}$ level $(\mathrm{dB})$ in the left channel

Finally, the experimental signals that had originally been recorded on a 12-bit sound card were converted to standard 16-bit “.WAV" files via custom software so that they could be played using a commercial sound card (Pro-audio Spectrum).

\subsection{Experimental Subjects}

Subjects consisted of five persons with normal hearing and no significant history of ear disease. All subjects were screened using a Beltone portable audiometer (Model 112) and found to have air conduction thresholds better than $15 \mathrm{~dB} \mathrm{HL}$ at $250,500,1000$, 
2000 and $4000 \mathrm{~Hz}$. The subjects ranged in age from 48 to 22 years with an average age of 33 years. Four of the subjects were female and one was male. All subjects were right handed.

\subsection{Procedure}

A simulated loudspeaker array was constructed and placed in a quiet, but not sound-treated, windowless test room in the basement of the Lexington School for the Deaf / Center for the Deaf. The array consisted of seventeen 7 -inch by $5 \frac{1}{4}$-inch cardboard boxes disguised as loudspeakers and mounted on iron pipes erected on a $180^{\circ}$ arc with a radius of 1 meter at intervals of $11.25^{\circ}$. Loudspeakers were labeled using the following protocol: 00 for the center loudspeaker, $\mathrm{L} 1$ to $\mathrm{L} 8$ for loudspeakers in the left quadrant, R1 to R8 for loudspeakers in the right quadrant. The subjects sat facing the center loudspeaker (labeled 00) in a dental chair that was placed at the center of the arc 1 meter from each of the simulated loudspeakers. The height of the chair was adjusted before each test session to ensure that the loudspeaker array and the ears of each subject lay on the same horizontal plane. A head-rest was provided so that subjects could place their heads in a reference position at the center of the arc of loudspeakers before each experimental trial. Subjects were observed during testing to ensure that they were facing the center loudspeaker as the test stimuli were presented. No other attempts were made to fix the head or prevent head movements during stimulus presentation.

The instrumentation for signal presentation is shown in Figure 9. Both channels of the binaural experimental signals were mixed in a Rane SM26 audio mixer with a dichotic white noise signal in which the right and left channels were uncorrelated. The digitally recorded experimental signals were reproduced by a Pro-Audio Spectrum Sound 
Card on a Pentium computer, and the white noise masker by a Panasonic SV-255 digital audio tape recorder. The mixed signals were presented to each subject via Etymotic ER-3A insert earphones attached to custom acrylic skeleton earmolds with standard \#13 tubing. The earmolds were fabricated with a large vent so that the ear canals were effectively open to the air. It was observed during informal listening that plugged ears reinforced the perception of an internalized signal. Vented earmolds were chosen in order to increase the subjects' perception that the signals were emanating from the dummy loudspeaker array. No systematic study of the externalization of the signals was undertaken, however. While vented earmolds affect the frequency response of signals in hearing aids, the effect is limited to the low frequencies and was not expected to affect the band-limited signals in this experiment (Mueller \& Hall, 1998). The presentation level of the stimuli was $80 \mathrm{~dB}$ SPL and the white noise masker was presented at $60 \mathrm{~dB}$ SPL. The purpose of the white noise masker was two-fold: 1) to mask any low-level environmental noises that may have appeared from time to time in the test room and 2) to mask the artifacts of adjusting the levels in the $50 \%$ attenuated condition and the $100 \%$ attenuated condition. The artifacts resulted from the fact that there was a low level of background noise in the recordings that was amplified or attenuated during the processing of the signals. These artifacts could have provided the subjects with additional cues that may have affected their localization judgments. It was found from informally listening to the processed stimuli in the test room that a $60 \mathrm{~dB}$ white noise was sufficient to mask processing artifacts. Yet this level of noise was low enough (20 $\mathrm{dB}$ signal to noise ratio) not to interfere with subjects' localization judgments in the horizontal plane (Good and Gilkey, 1996). 


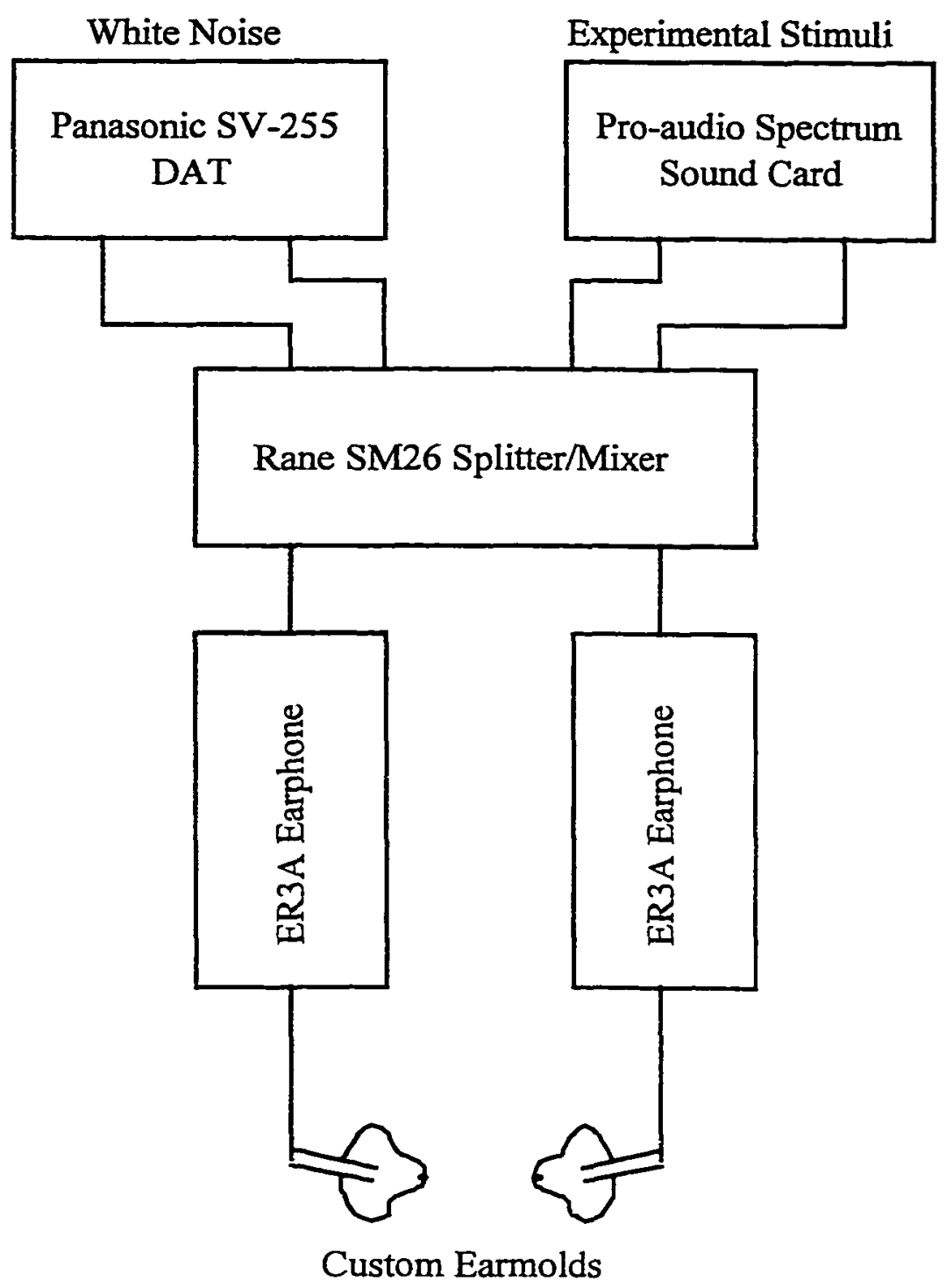

Figure 9: Instrumentation for subject testing.

Experimental testing consisted of a sound source identification task. Subjects sat facing loudspeaker 00 , listened to each stimulus and called out the number of the loudspeaker position from which the sound apparently originated. For trials in which subjects were unsure of their response, they were permitted to request as many repetitions of the stimulus as they needed before making a judgment. Subjects made five independent localization judgments for each loudspeaker location, except for the $0^{\circ}$ 
azimuth loudspeaker location, for which they made ten localization judgments. The number of replications at $0^{\circ}$ azimuth was doubled to allow for the possibility of collapsing the data from the left and right sides. (As it turned out, this was not possible because of the asymmetries that occurred in the results.) No feedback was provided during the test sessions, although feedback was provided during training rounds using only unmodified test signals. The rationale for withholding feedback was limit the possibility of subjects' adapting to the interaural intensity difference modifications over the test sessions, which would result in the addition of another variable to the experiment, i.e., learning. A single test round consisted of 90 sound source identifications: 5 replications at each of the 16 non-zero azimuths and 10 replications at $0^{\circ}$ azimuth. Test rounds were blocked on signal type (7), room type (2), and interaural intensity difference condition (3) in that order. Thus, all testing for a given signal type in both rooms and all three interaural intensity difference conditions was completed before moving on to next signal type. The sequence of testing was randomly ordered across all variables within and between test blocks.

Each subject was trained on the localization task prior to data collection. A minimum of one training round was provided before testing began with each new signal type. A training round consisted of a single replication, with feedback, at each of the 16 non-zero azimuths and two replications at $0^{\circ}$ azimuth. The training was performed using the unattenuated signals only. Feedback consisted of a recorded voice indicating the actual sound source location after each localization trial. Training rounds continued until the subject indicated that he or she was comfortable with the task. Informal piloting prior 
to the conducting the experiment indicated that performance was stable and did not improve after several repetitions of the training task. 


\section{CHAPTER 4: RESULTS}

\subsection{Source-response Scatterplots}

The data resulting from this experiment consist of a series of auditory localization judgments. A convenient way to view these data is by the use of a scatterplot with source loudspeaker location plotted on the abscissa and response loudspeaker location on the ordinate. Shown in Figure 10 and Figure 11 are scatterplots that show the effect of interaural intensity difference condition on localization judgments at $0.5 \mathrm{kHz}$ (the smallest effect) and $2.0 \mathrm{kHz}$ (the greatest effect), respectively. In addition, scatterplots of the entire data set for each signal type and frequency under each of the interaural intensity difference conditions under evaluation in this experiment are shown in the Appendix in Figure 50 through Figure 70. The data are plotted in the form of "sunflowers" that have a center and one or more "petals." One case within each cell on the graph is represented by a sunflower center, and each additional case within that cell is represented by one petal (SPSS Inc., 1997). ${ }^{1}$ In these figures, loudspeakers are identified by number with zero representing the center, negative numbers indicating the left side and positive numbers the right side. These plots provide an overview of the data that allows one to observe general trends for the accuracy of localization under different conditions as well as the occurrence of left/right reversals and outliers. If the localization judgments were without error, all data points would lie on the diagonal of the scatter plot. The vertical distance of a given data point from the diagonal is a measure of the localization error. Data points lying above the diagonal for negative azimuths (left side) or below the diagonal for positive azimuths (right side) represent left/right reversals.

\footnotetext{
'Cells on a sunflower scatterplot are arranged so that the range of the data is broken up into 15 cells. Thus, there are fewer columns and rows than one would expect for a $17 \times 17$ matrix.
} 
Left/right reversals occur when a subject responds to a signal whose source is on the left with a response to the right, or visa versa. Note that twice as many observations were made at loudspeaker 0 than at any other location. This was done so that responses on the left side could be conveniently compared to those on the right when analyzing the results of the experiment.

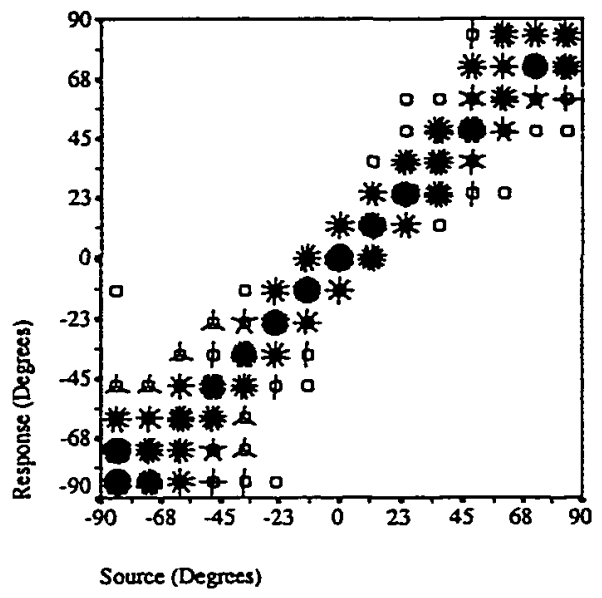

a) Natural IID

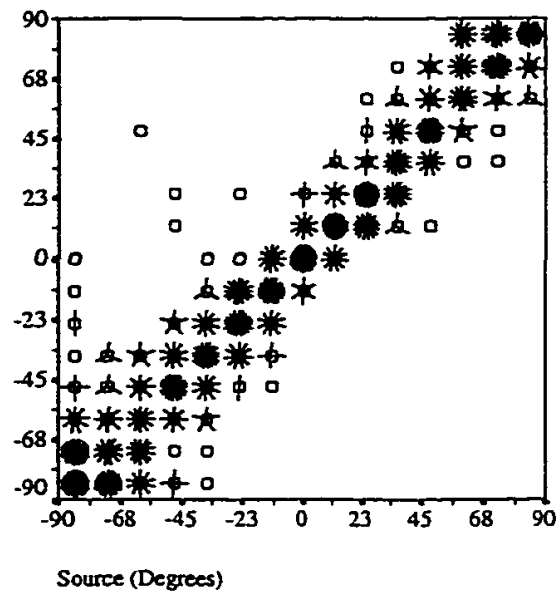

b) $50 \%$ attenuated $\mathrm{IDD}$

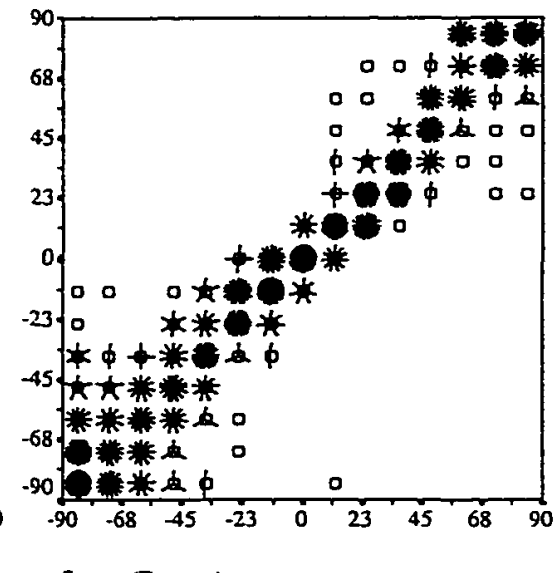

Source (Degrees)

c) $100 \%$ attenuated $\mathrm{ID}$

Figure 10: Scatterplots showing the gross effect of interaural intensity difference condition on auditory localization performance for $0.5 \mathrm{kHz}$ third-octave bands of noise. 


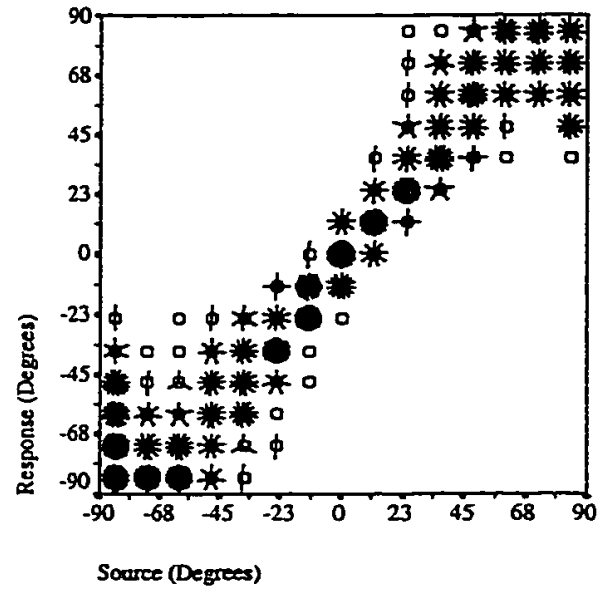

a) Natural IID

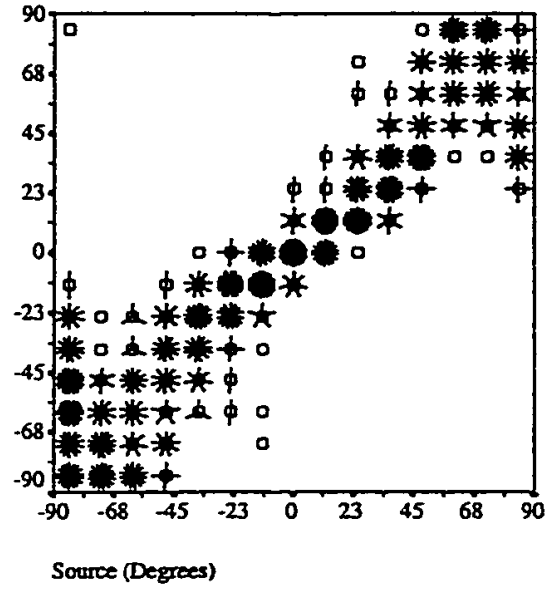

b) $50 \%$ attenuated $\mathrm{ID}$

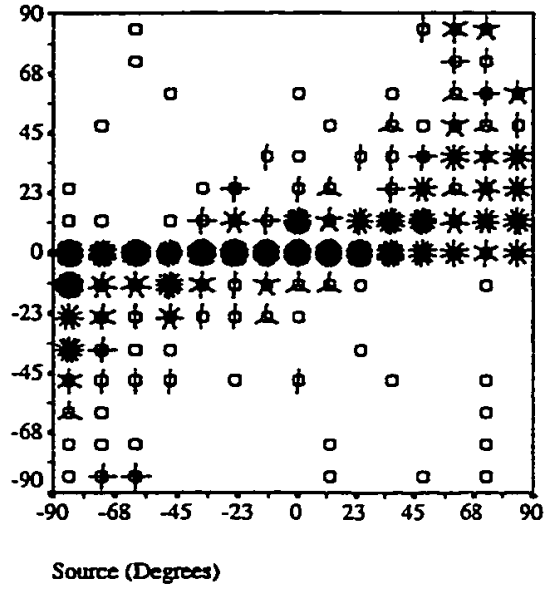

c) $100 \%$ attenuated IID

Figure 11: Scatterplots showing the gross effect of interaural intensity difference condition on auditory localization performance for $2.0 \mathrm{kHz}$ third-octave bands of noise.

\subsubsection{Left/Right Reversals}

It can be seen in the scatterplots above and in the Appendix that the number of left/right reversals was much greater for the $100 \%$ attenuated condition than for the other two interaural intensity difference conditions. A tally was made of the number of times that left/right reversals occurred for each frequency and interaural intensity difference condition. A reversal was defined as a response on the opposite side of the target speaker location where the difference between the target and the response was greater than 2 . The results of the tally are shown in Figure 12. While there was a small number of leftright reversals for the unattenuated and $50 \%$ attenuated conditions, the vast majority of left/right reversals occurred in the $100 \%$ attenuated condition. There were many fewer left/right reversals for the $0.5 \mathrm{kHz}$ third-octave band and the 0.5 to $4.0 \mathrm{kHz}$ band-pass noise than there were for signals with no low-frequency content. In addition, the number 
of left/right reversals was much greater for the high-frequency third-octave bands than for the high-frequency signals with wider bandwidth.

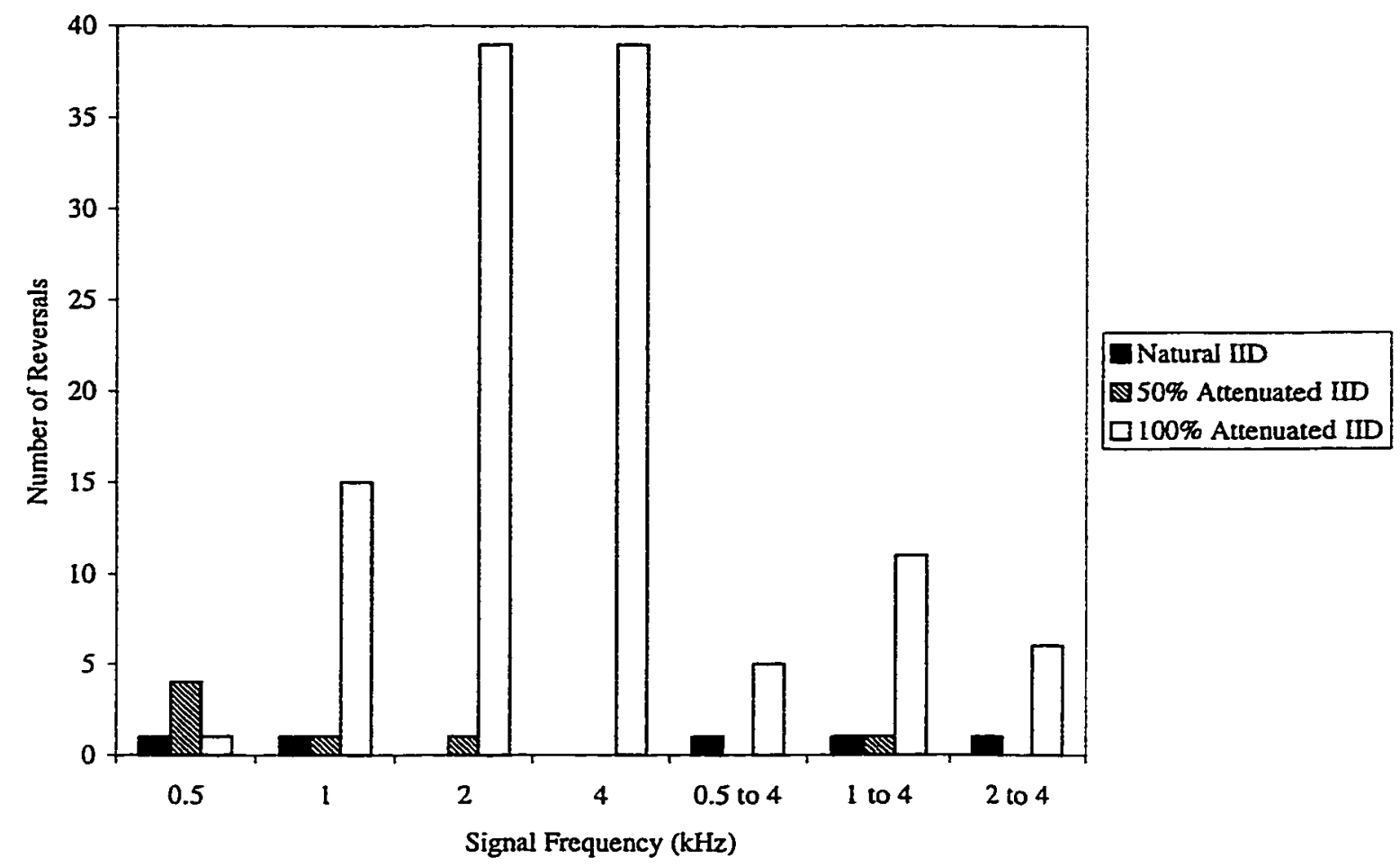

Figure 12: Number of left/right reversals for each signal type and interaural intensity difference condition.

\subsection{Derivation of Measures for Quantifying Localization Error}

Each localization judgment in this experiment can be thought of as consisting of two factors: the true loudspeaker location and an error. This relationship is represented as

$$
x_{i}=x_{t}+e_{i}
$$

Equation 7

where $x_{t}$ is the true loudspeaker location and $e_{i}$ is the error term. The error term consists of two components

$$
e_{i}=E_{b}+E_{i}
$$


where $E_{b}$ represents the bias and $E_{l}$ represents the test-retest random error. The bias $\left(E_{b}\right)$ is a fixed quantity for each cell in the experiment. It is assumed that the test-retest random error $\left(\boldsymbol{E}_{\boldsymbol{l}}\right)$ is normally distributed with a mean equal to zero and a standard deviation $\sigma_{E}$, the test-retest standard deviation. Substituting Equation 8 for $e_{i}$ in Equation 7 yields

$$
x_{i}=x_{t}+E_{b}+E_{i}
$$

Equation 9

Thus, each localization judgment within a cell is composed of the true loudspeaker location $\left(\boldsymbol{x}_{t}\right)$, the bias $\left(\boldsymbol{E}_{b}\right)$ and a test-retest random error from which a measure of precision can be derived.

The mean of $\mathrm{n}$ localization judgments within a cell can be expressed as

$$
\bar{x}=\frac{1}{n}\left[x_{1}+x_{2}+\ldots+x_{i}+\ldots+x_{n}\right]
$$

Substituting Equation 9 for $\boldsymbol{x}_{\boldsymbol{i}}$ yields

$$
\begin{aligned}
& \bar{x}=\frac{1}{n}\left[\left(x_{t}+E_{b}+E_{1}\right)+\left(x_{t}+E_{b}+E_{2}\right)+\ldots+\left(x_{t}+E_{b}+E_{i}\right)+\ldots+\left(x_{t}+E_{b}+E_{n}\right)\right] \\
& \bar{x}=\frac{1}{n}\left[n x_{t}+n E_{b}+\left(E_{1}+E_{2}+\ldots+E_{i}+\ldots+E_{n}\right)\right] \\
& \bar{x}=x_{t}+E_{b}+\frac{1}{n}\left(E_{1}+E_{2}+\ldots+E_{i}+\ldots+E_{n}\right)
\end{aligned}
$$$$
\bar{x}=x_{t}+E_{b}+\bar{E}
$$

Equation 11

where $\bar{E}$ is the mean error. $\bar{E}$ is a random variable, smaller than $E_{i}$ on average. For a stable statistical distribution,

$$
\sigma_{\bar{E}}=\frac{1}{\sqrt{n}} \sigma_{E}
$$

Equation 12 
where $\sigma_{\bar{E}}$ is the standard error of the mean and $\sigma_{E}$ is the test-retest standard deviation.

\subsubsection{Precision of Localization}

An estimate of the precision of localization for a single judgment $\left(\sigma_{E}\right)$ can be calculated as follows:

$$
\begin{aligned}
& \hat{\sigma}_{E}^{2}=\frac{1}{n-1} \sum_{i=1}^{n}\left(x_{i}-\bar{x}\right)^{2} \\
& \hat{\sigma}_{E}^{2}=\frac{1}{n-1} \sum_{i=1}^{n}\left[\left(x_{t}+E_{b}+E_{i}\right)-\left(x_{t}+E_{b}+\bar{E}\right)\right]^{2} \\
& \hat{\sigma}_{E}^{2}=\frac{1}{n-1} \sum_{i=1}^{n}\left[\left(x_{t}-x_{t}\right)+\left(E_{b}-E_{b}\right)+\left(E_{i}-\bar{E}\right)\right]^{2} \\
& \hat{\sigma}_{E}^{2}=\frac{1}{n-1} \sum_{i=1}^{n}\left(E_{i}-\bar{E}\right)^{2}
\end{aligned}
$$

yielding

$$
\hat{\sigma}_{E}=\sqrt{\frac{1}{n-1} \sum_{i=1}^{n}\left(E_{i}-\bar{E}\right)^{2}}
$$

Note that the bias $E_{b}$ has been canceled out. This estimate of the test-retest standard deviation, $\hat{\sigma}_{E}$, serves as an unbiased estimate of the precision of the localization judgments within a cell.

\subsubsection{Bias}

As $\mathrm{n}$ increases in an experiment, the estimate of the test-retest standard deviation, $\hat{\sigma}_{E}$, becomes more accurate. In addition, $\sigma_{\bar{E}} \Rightarrow \mathbf{0}$ (see Equation 12) and $\bar{E} \Rightarrow 0$ (because $\bar{E}=\frac{1}{n} \sum E_{i}$ ). From Equation 11, for a large n, $\bar{x} \approx x_{t}+E_{b}$ and 


$$
E_{b} \approx \bar{x}-x_{t}
$$

Equation 14

The accuracy with which the bias, $\boldsymbol{E}_{\mathbf{b}}$, can be estimated is limited by the random error term $E_{1}$. Equation 14 provides a means for estimating $E_{b}$ in which the effect of the random error terms is minimized.

\subsubsection{Accuracy of Localization}

Hartmann (1983) regards root mean square error, $\sigma_{e}$, as "the most meaningful single number to describe localization performance," where

$$
\sigma_{e}=\sqrt{\frac{1}{n} \sum_{i=1}^{n}\left[x_{i}-x_{t}\right]^{2}}
$$

Equation 15

The root mean square error $\left(\sigma_{e}\right)$ expresses the overall accuracy of localization, including the effects of both bias $\left(\boldsymbol{E}_{b}\right)$ and precision $\left(\sigma_{E}\right)$. Equation 16 shows the relationship among overall accuracy $\left(\sigma_{e}\right)$, bias $\left(\boldsymbol{E}_{b}\right)$ and precision of localization $\left(\sigma_{E}\right)$.

$$
\sigma_{e}=\sqrt{E_{b}^{2}+\frac{n-1}{n} \sigma_{E}^{2}}
$$

Equation 16

\subsubsection{Summary of Measures for Quantifying Localization Error}

In summary, there are three measures that will be used in the analysis of the results of this experiment: root mean square error $\left(\sigma_{e}\right)$, a measure of localization accuracy, bias $\left(\boldsymbol{E}_{\boldsymbol{b}}\right)$ and test-retest standard deviation $\left(\sigma_{E}\right)$, a measure of the precision of localization judgments. These measures are similar to those suggested by Hartman (1983) to evaluate listeners' localization performance. Each of the three measures was calculated over the 5 independent localization judgments for each of the 5 subjects and each of the independent variables, i.e., loudspeaker location, signal type, interaural intensity difference condition and room. For convenience, all measures were calculated in 
units of loudspeaker location, where 1 loudspeaker location equals $11.25^{\circ}$. All measures of the data reported in this dissertation have been converted back into degrees.

\subsection{Root Mean Square Error $\left(\sigma_{e}\right)$ : Accuracy of Localization}

\section{A General Linear Model Repeated Measures Analysis of Variance (ANOVA)}

(SPSS Advanced Statistics ${ }^{\text {TM }} 7.5,1997$ ) was performed separately for each signal category (third-octave band noise and band-pass noise) over the dependent variable root mean square error $\left(\sigma_{e}\right)$. The factors for the analysis were interaural intensity difference condition (ID, 3 levels), signal type (FREQ, 4 levels for third-octave bands, 3 levels for band-pass noises), loudspeaker location (AZIM, 18 levels $^{2}$ ) and room (ROOM, 2 levels), with repeated measures over 5 subjects. The ANOVA tables for these analyses appear in Table 2 and Table 3.

\footnotetext{
${ }^{2}$ There were 17 speaker locations in all. All measures $\left(\sigma_{e}, \boldsymbol{E}_{b}\right.$ and $\left.\sigma_{E}\right)$ were calculated twice for the center loudspeaker location since ten trials were made there, twice as many as at any other location. (AZIM could have been subdivided into two factors, angle and side [left or right]. It was for this reason that twice as many observations were obtained at $\mathrm{AZIM}=0$.) The first 5 trials were used to calculate the first measure and the second 5 used to calculate the second measure.
} 
Table 2: ANOVA table of root mean square error for third-octave bands of noise. IID represents interaural intensity difference condition, FREQ represents signal type, AZIM represents loudspeaker location and ROOM represents room type.

\begin{tabular}{|c|c|c|c|c|c|}
\hline \multicolumn{6}{|c|}{ Tests of Within-Subjects Effects } \\
\hline \multicolumn{6}{|c|}{ Root Mean Square Error - Third-octave Bands } \\
\hline & Sphericity & sumed & & & \\
\hline Source & $\begin{array}{l}\text { Type III } \\
\text { Sum of } \\
\text { Squares }\end{array}$ & $\mathrm{df}$ & $\begin{array}{l}\text { Mean } \\
\text { Square }\end{array}$ & $\mathrm{F}$ & Sig. \\
\hline III & 417.455 & 2 & 208.728 & 58.593 & 0.000 \\
\hline Error(IID) & 28.499 & 8 & 3.562 & & \\
\hline FREQ & 430.292 & 3 & 143.431 & 31.374 & 0.000 \\
\hline Error(FREQ) & 54.860 & 12 & 4.572 & & \\
\hline AZIM & 614.970 & 17 & 36.175 & 17.207 & 0.000 \\
\hline Error(AZIM) & 142.960 & 68 & 2.102 & & \\
\hline ROOM & 0.143 & 1 & 0.143 & 0.048 & 0.838 \\
\hline Error(ROOM) & 12.052 & 4 & 3.013 & & \\
\hline IID * FREQ & 285.757 & 6 & 47.626 & 56.525 & 0.000 \\
\hline Error(III * FREQ) & 20.222 & 24 & 0.843 & & \\
\hline III * AZIM & 144.885 & 34 & 4.261 & 5.837 & 0.000 \\
\hline Error(IID*AZIM) & 99.281 & 136 & 0.730 & & \\
\hline IID * ROOM & 0.571 & 2 & 0.286 & 0.139 & 0.872 \\
\hline Error(IID * ROOM) & 16.390 & 8 & 2.049 & & \\
\hline FREQ * AZIM & 395.948 & 51 & 7.764 & 9.158 & 0.000 \\
\hline Error(FREQ*AZIM) & 172.933 & 204 & 0.848 & & \\
\hline FREQ * ROOM & 3.984 & 3 & 1.328 & 0.994 & 0.429 \\
\hline Error(FREQ * ROOM) & 16.036 & 12 & 1.336 & & \\
\hline AZIM * ROOM & 13.610 & 17 & 0.801 & 1.270 & 0.239 \\
\hline Error(AZIM * ROOM) & 42.876 & 68 & 0.631 & & \\
\hline IID * FREQ * AZIM & 159.600 & 102 & 1.565 & 3.187 & 0.000 \\
\hline Error(IID * FREQ * AZIM) & 200.294 & 408 & 0.491 & & \\
\hline IID * FREQ * ROOM & 5.197 & 6 & 0.866 & 1.294 & 0.298 \\
\hline Error(II * FREQ * ROOM) & 16.067 & 24 & 0.669 & & \\
\hline IID * AZIM * ROOM & 17.694 & 34 & 0.520 & 1.233 & 0.200 \\
\hline $\operatorname{Error}(\mathrm{ID} *$ AZIM * ROOM $)$ & 57.378 & 136 & 0.422 & & \\
\hline FREQ * AZIM * ROOM & 34.872 & 51 & 0.684 & 1.352 & 0.075 \\
\hline Error(FREQ * AZIM * ROOM) & 103.189 & 204 & 0.506 & & \\
\hline IID * FREQ * AZIM * ROOM & 62.654 & 102 & 0.614 & 1.640 & 0.000 \\
\hline $\begin{array}{l}\text { Error(IID * FREQ * AZTM * } \\
\text { ROOM) }\end{array}$ & 152.853 & 408 & 0.375 & & \\
\hline
\end{tabular}


Table 3: ANOVA table of root mean square error for band-pass noises. IID represents interaural intensity difference condition, FREQ represents signal type, AZIM represents loudspeaker location and ROOM represents room type.

\begin{tabular}{|c|c|c|c|c|c|}
\hline \multicolumn{6}{|c|}{ Tests of Within-Subjects Effects } \\
\hline \multicolumn{6}{|c|}{ Root Mean Square Error - Band-pass Noises } \\
\hline \multicolumn{6}{|c|}{ Sphericity Assumed } \\
\hline Source & $\begin{array}{l}\text { Type III } \\
\text { Sum of } \\
\text { Squares }\end{array}$ & df & $\begin{array}{l}\text { Mean } \\
\text { Square }\end{array}$ & $\overline{\mathrm{F}}$ & Sig. \\
\hline IID & 125.664 & 2 & 62.832 & 26.017 & 0.000 \\
\hline Error(IID) & 19.321 & 8 & 2.415 & & \\
\hline FREQ & 69.692 & 2 & 34.846 & 7.661 & 0.014 \\
\hline Error(FREQ) & 36.387 & 8 & 4.548 & & \\
\hline$\widehat{\text { AZIM }}$ & 417.769 & 17 & 24.575 & 12.945 & 0.000 \\
\hline Error(AZIM) & 129.088 & 68 & 1.898 & & \\
\hline ROOM & 1.055 & $\overline{1}$ & 1.055 & 0.673 & 0.458 \\
\hline Error(ROOM) & 6.267 & $\overline{4}$ & 1.567 & & \\
\hline III * FREQ & 1.467 & $\overline{4}$ & 0.367 & 0.158 & 0.956 \\
\hline Error(IID * FREQ) & 37.093 & 16 & 2.318 & & \\
\hline IID * AZIM & 261.159 & 34 & 7.681 & 10.224 & 0.000 \\
\hline Error(III*AZIM) & 102.178 & 136 & 0.751 & & \\
\hline III * ROOM & 0.880 & 2 & 0.440 & 0.618 & 0.563 \\
\hline Error(IID * ROOM) & 5.691 & 8 & 0.711 & & \\
\hline FREQ * AZIM & 42.111 & 34 & 1.239 & 2.052 & 0.002 \\
\hline Error(FREQ*AZIM) & 82.073 & 136 & 0.603 & & \\
\hline FREQ * ROOM & 3.969 & 2 & 1.985 & 4.025 & 0.062 \\
\hline Error(FREQ * ROOM) & 3.945 & 8 & 0.493 & & \\
\hline AZIM * ROOM & 8.475 & 17 & 0.499 & 1.357 & 0.186 \\
\hline Error(AZIM * ROOM) & 24.983 & 68 & 0.367 & & \\
\hline III * FREQ * AZIM & 56.073 & 68 & 0.825 & 1.795 & 0.001 \\
\hline Error(IID * FREQ * AZIM) & 124.979 & 272 & 0.459 & & \\
\hline III * FREQ * ROOM & 4.521 & 4 & 1.130 & 2.197 & 0.116 \\
\hline Error(m ${ }^{*}$ FREQ $*$ ROOM $)$ & 8.230 & 16 & 0.514 & & \\
\hline III * AZIM * ROOM & 17.897 & 34 & 0.526 & 1.887 & 0.006 \\
\hline Error(IID * AZIM * ROOM) & 37.938 & 136 & 0.279 & & \\
\hline FREQ * AZIM * ROOM & 16.308 & 34 & 0.480 & 1.605 & 0.030 \\
\hline Error(FREQ * AZIM * ROOM) & 40.632 & 136 & 0.299 & & \\
\hline III * FREQ * AZIM * ROOM & 28.007 & 68 & 0.412 & 1.529 & 0.010 \\
\hline $\begin{array}{l}\text { Error(IID * FREQ * AZIM * } \\
\text { ROOM) }\end{array}$ & 73.268 & 272 & 0.269 & & \\
\hline
\end{tabular}




\subsubsection{Interaural Intensity Difference Condition}

There were three conditions for interaural intensity difference. In the first condition, the interaural intensity difference was not modified from its original value when recorded on KEMAR. In the second condition, the interaural intensity difference was attenuated by $50 \%$ in $\mathrm{dB}$ so that it became one half of its original root mean square value in decibels. In the third condition, the left and right channels were attenuated to be equal in root mean square level in $\mathrm{dB}$, i.e., the interaural intensity differences were attenuated by $100 \%$.

The main effect of interaural intensity difference condition on root mean square error is shown in Figure 13. Results of the Tukey HSD (honestly significant difference) test are displayed on this graph (and all of the figures that follow) by error bars that represent the magnitude of the HSD at a $95 \%$ level of confidence. Where the distance between points on the graph exceed the length of the error bars, a statistically significant $(p<0.05)$ difference exists. 


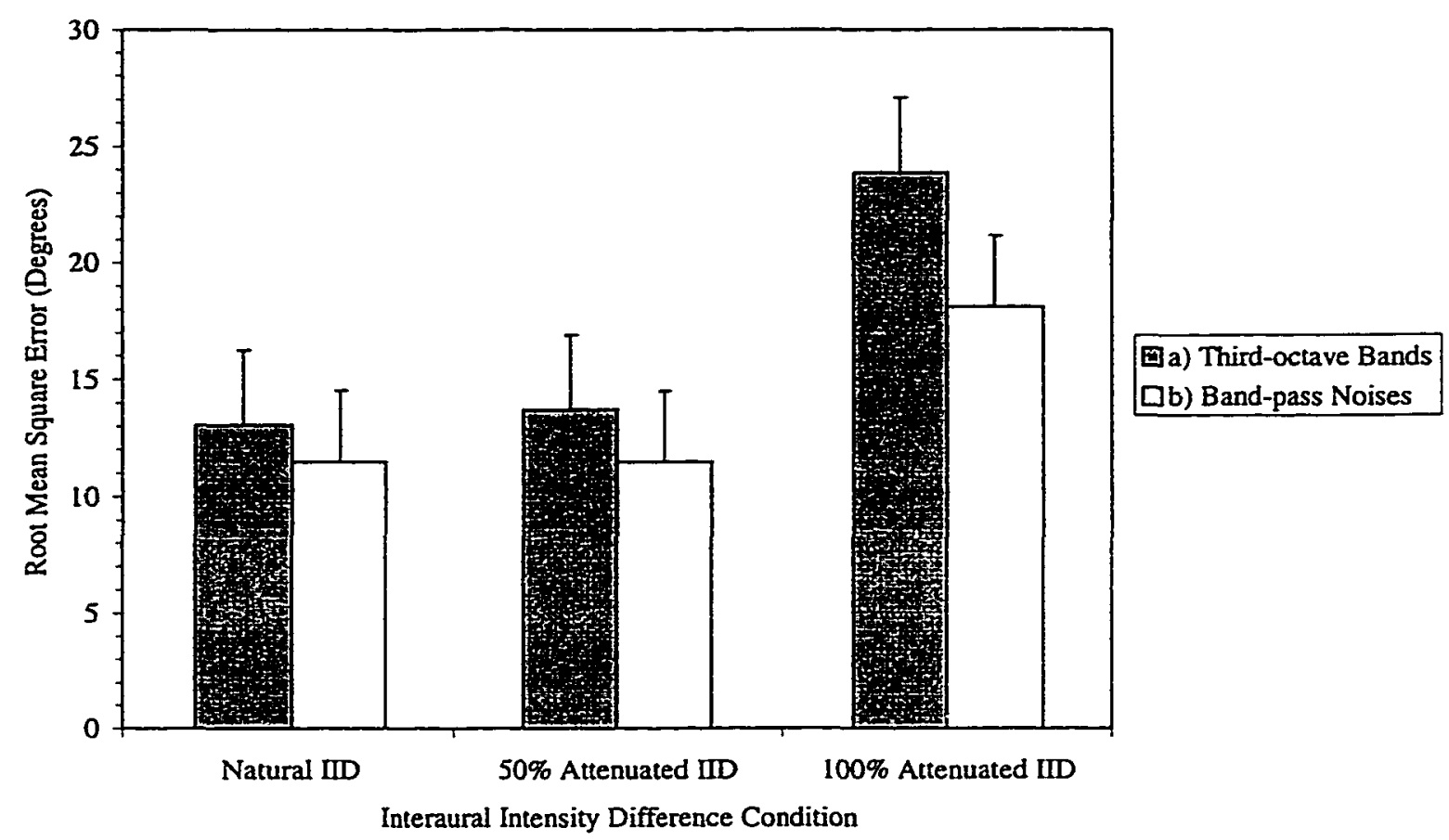

Figure 13: Effect of interaural intensity difference condition on root mean square error. Error bars represent a difference at the 0.05 level of significance using Tukey's HSD.

The figure shows that attenuation of the interaural intensity differences by $100 \%$ resulted in greater root mean square error magnitude for both third-octave and band-pass noises. There was no significant difference between the unattenuated and $50 \%$ attenuated conditions, however.

\subsubsection{Signal Frequency}

Signal frequency was a significant factor in the analyses for both third-octave bands of noise and band-pass noises. The effect of signal frequency is illustrated in Figure 14. 


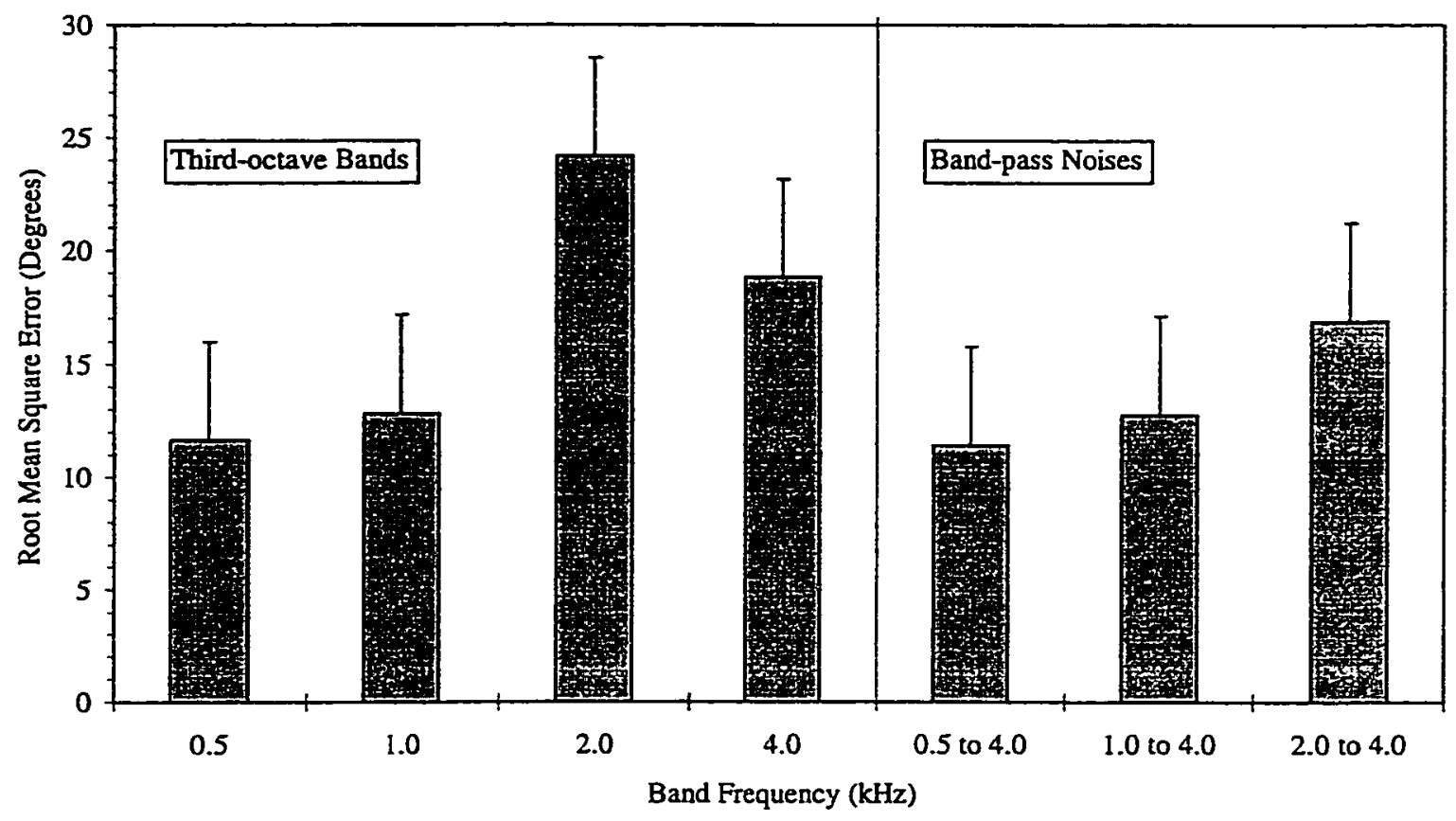

Figure 14: Effect of signal frequency on root mean square error. Error bars represent a difference at the 0.05 level of significance using Tukey's HSD.

For the third-octave bands, subjects demonstrated greater root mean square error when localizing the bands of higher center frequency. There was a significantly increased root mean square error for $2.0 \mathrm{kHz}$ and $4.0 \mathrm{kHz}$ relative to the other noises with lower center frequencies. Interestingly, root mean square errors at $2.0 \mathrm{kHz}$ were significantly greater than those at $4.0 \mathrm{kHz}$. Root mean square errors for $0.5 \mathrm{kHz}$ and $1.0 \mathrm{kHz}$ were not different from one another.

A similar pattern can be seen in the results for the band-pass noises. There was increased error for the $2.0 \mathrm{kHz}$ to $4.0 \mathrm{kHz}$ band-pass noise relative to the 0.5 to $4.0 \mathrm{kHz}$ band-pass noise, but the root mean square errors for the 0.5 to $4.0 \mathrm{kHz}$ and 1.0 to $4.0 \mathrm{kHz}$ bands were not different. 


\subsubsection{Interaural Intensity Difference Condition and Signal Frequency}

The effects of interaural intensity difference condition on third-octave bands with different center frequencies and band-pass noises with different frequency content can be seen in Figure 15.

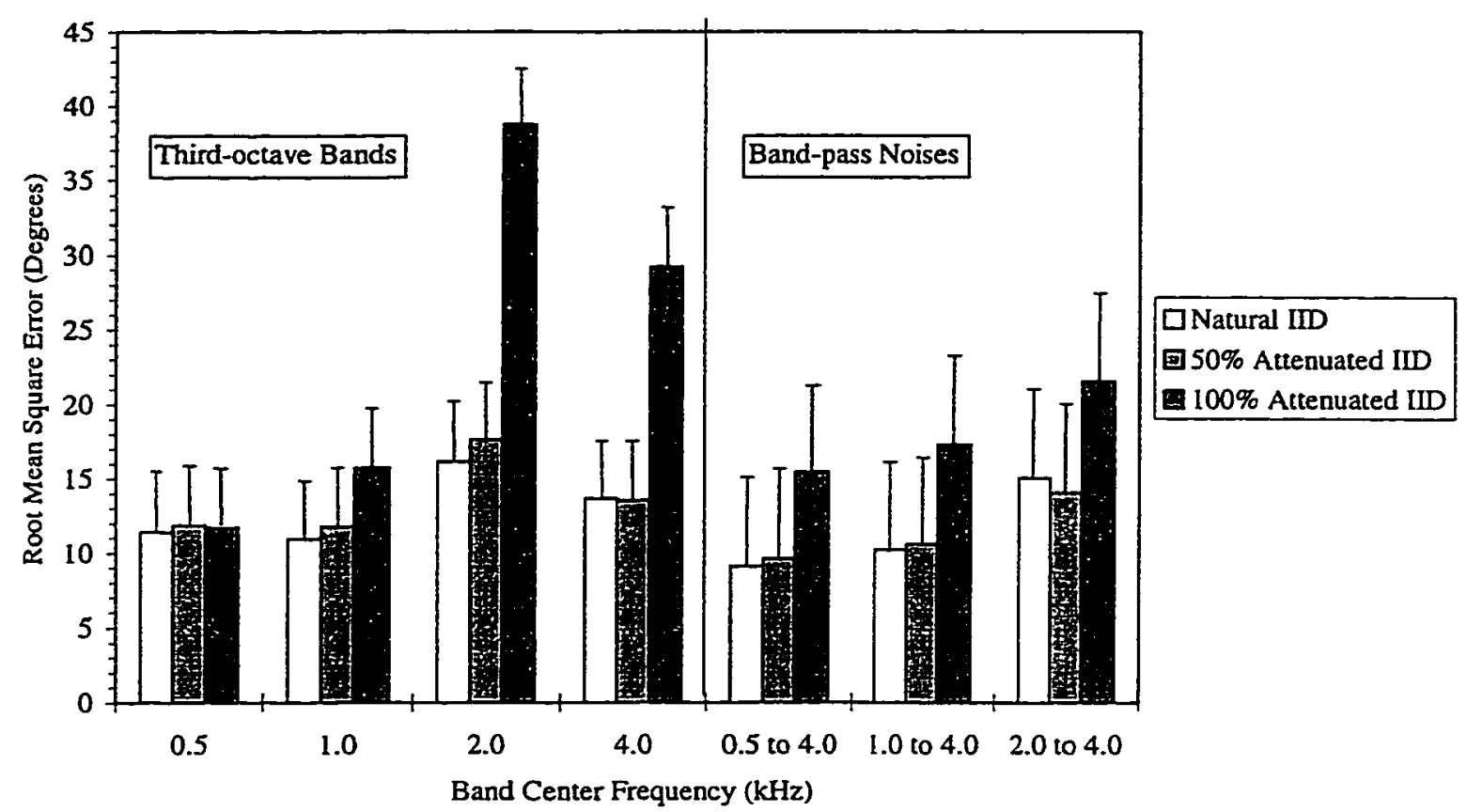

Figure 15: Effect of interaural intensity difference condition and signal frequency on root mean square error. Error bars represent a difference at the 0.05 level of significance using Tukey's HSD.

For the third-octave band noises, there was a significant interaction between signal frequency and interaural intensity difference condition, indicating that the attenuation of interaural intensity differences affected the localization of signals of different frequency differently. Clearly, modifications of the interaural intensity difference had no effect on the localization of $0.5 \mathrm{kHz}$ third-octave noise bands. The localization of $1.0 \mathrm{kHz}$ third-octave noise bands was affected by $100 \%$ attenuation of interaural intensity differences relative to the unattenuated condition, but not by $50 \%$ 
attenuation. The same is true for third-octave band noises with center frequencies of 2.0 $\mathrm{kHz}$ and $4.0 \mathrm{kHz}$. The greatest magnitude of errors in the $100 \%$ attenuation condition occurred with $2.0 \mathrm{kHz}$ third-octave band noises. As shown by the error bars, for the 2 and $4.0 \mathrm{kHz}$ noises, there was a significant difference between root mean square errors for the $100 \%$ attenuated condition versus the $50 \%$ attenuated and unattenuated conditions, and no difference between the $50 \%$ attenuated and unattenuated conditions. For the $1.0 \mathrm{kHz}$ noise, there was a significant increase in errors for $100 \%$ attenuated relative to unattenuated, but not relative to $50 \%$ attenuated.

\subsubsection{Azimuth}

Figure 16 shows the relationships between angle of azimuth of the sound source and root mean square error for third-octave bands and band limited noises. Azimuth was a significant main effect in both analyses.

The magnitude of root mean square error tended to increase with increasing distance from the center $\left(0.00^{\circ}\right)$. Loudspeaker locations on equal and opposite sides of $0.00^{\circ}$ showed no significant differences for the third-octave bands, but differences between loudspeaker locations at $90^{\circ}, 78^{\circ}$ and $67.5^{\circ}$ to the right and left of $0.00^{\circ}$ were significant for the band-pass noises. These differences provide statistical evidence for asymmetry in the main effect for Azimuth, i.e., errors for the band-pass noises were greater on the left side than on the right for azimuths greater than $67.50^{\circ}$.

Azimuth $0.00^{\circ}$ is indicated twice on the figure because there were twice as many replications at that angle of azimuth as for the other loudspeaker locations. The root mean square error for $0.00^{\circ}$ was calculated twice, the first (on the left in the figure) with the first five replications for each test and the second with the second five replications. 


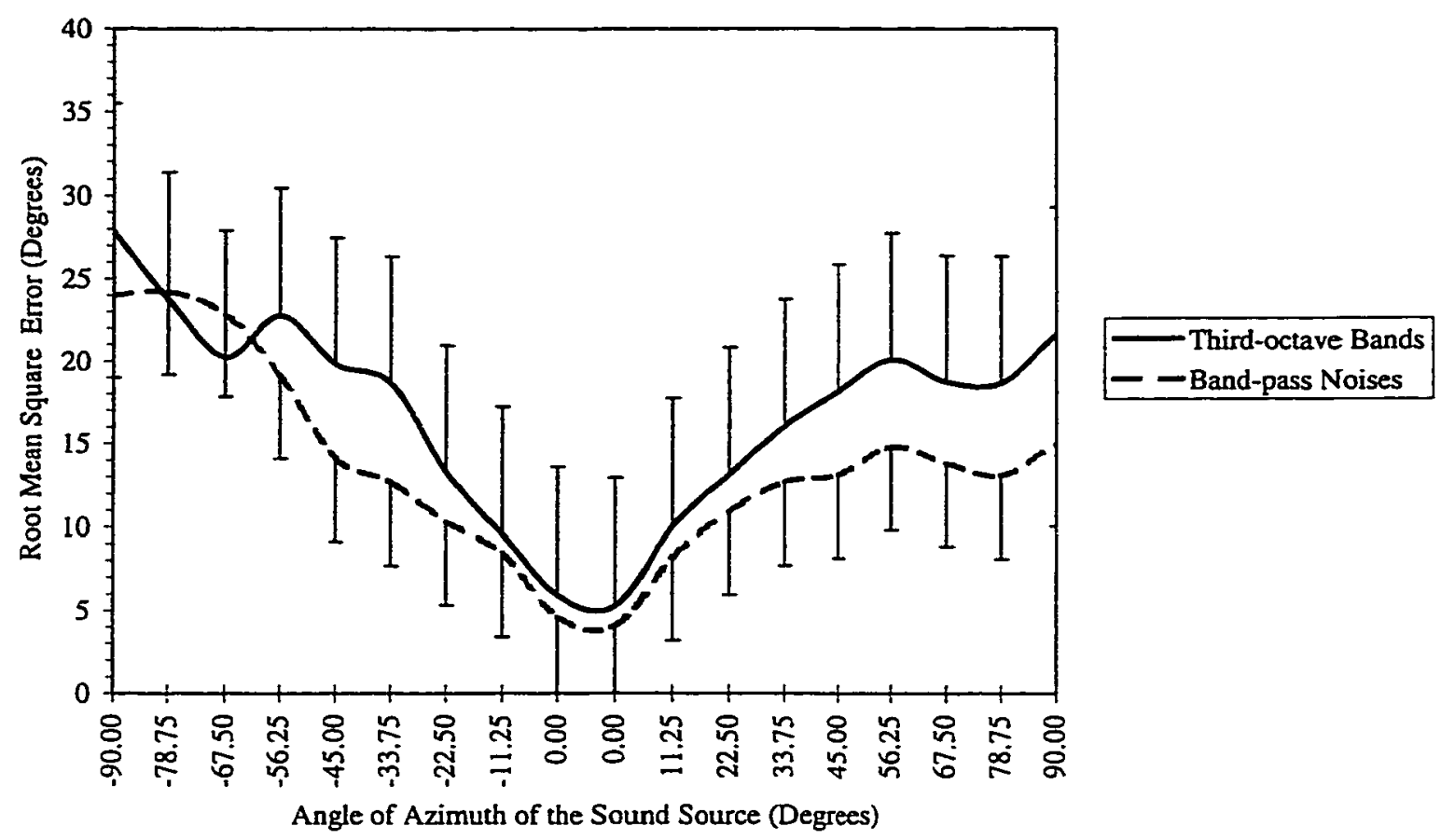

Figure 16: Effect of sound source azimuth on root mean square error. Error bars represent a difference at the $\mathbf{0 . 0 5}$ level of significance using Tukey's HSD. For clarity of presentation, error bars are shown only in a positive direction for thirdoctave bands and in a negative direction for band-pass noises.

\subsubsection{Azimuth and Signal Frequency}

There were significant interactions between the factors of azimuth and signal frequency for both third-octave band noises and band-pass noises. These interactions are shown as line plots in Figure 17.

The plots of root mean square error over azimuth differ in shape among signal frequencies. There is little difference between the plots for $0.5 \mathrm{kHz}$ and $1.0 \mathrm{kHz}$ thirdoctave band noises and the 0.5 to $4.0 \mathrm{kHz}$ and 1.0 to $4.0 \mathrm{kHz}$ band-pass noises. These are plotted as thin solid lines in Figure 17 . The plot for the $2.0 \mathrm{kHz}$ third-octave band however, where the error level rises sharply with azimuth out to $90^{\circ}$, stands out clearly as 
different from the others, and is plotted as a thick solid line. The plot for $4.0 \mathrm{kHz}$, shown as a thick dashed line, closely resembles that for $2.0 \mathrm{kHz}$ until an azimuth of $67.5^{\circ}$, beyond which the errors for $2.0 \mathrm{kHz}$ rise sharply at the most extreme azimuths. For the 2.0 to $4.0 \mathrm{kHz}$ band-pass noise, shown as a thick dotted line, the root mean square errors are elevated relative to the other band-pass noises at two points: $90^{\circ}$ to the left and $78.75^{\circ}$ to the right.

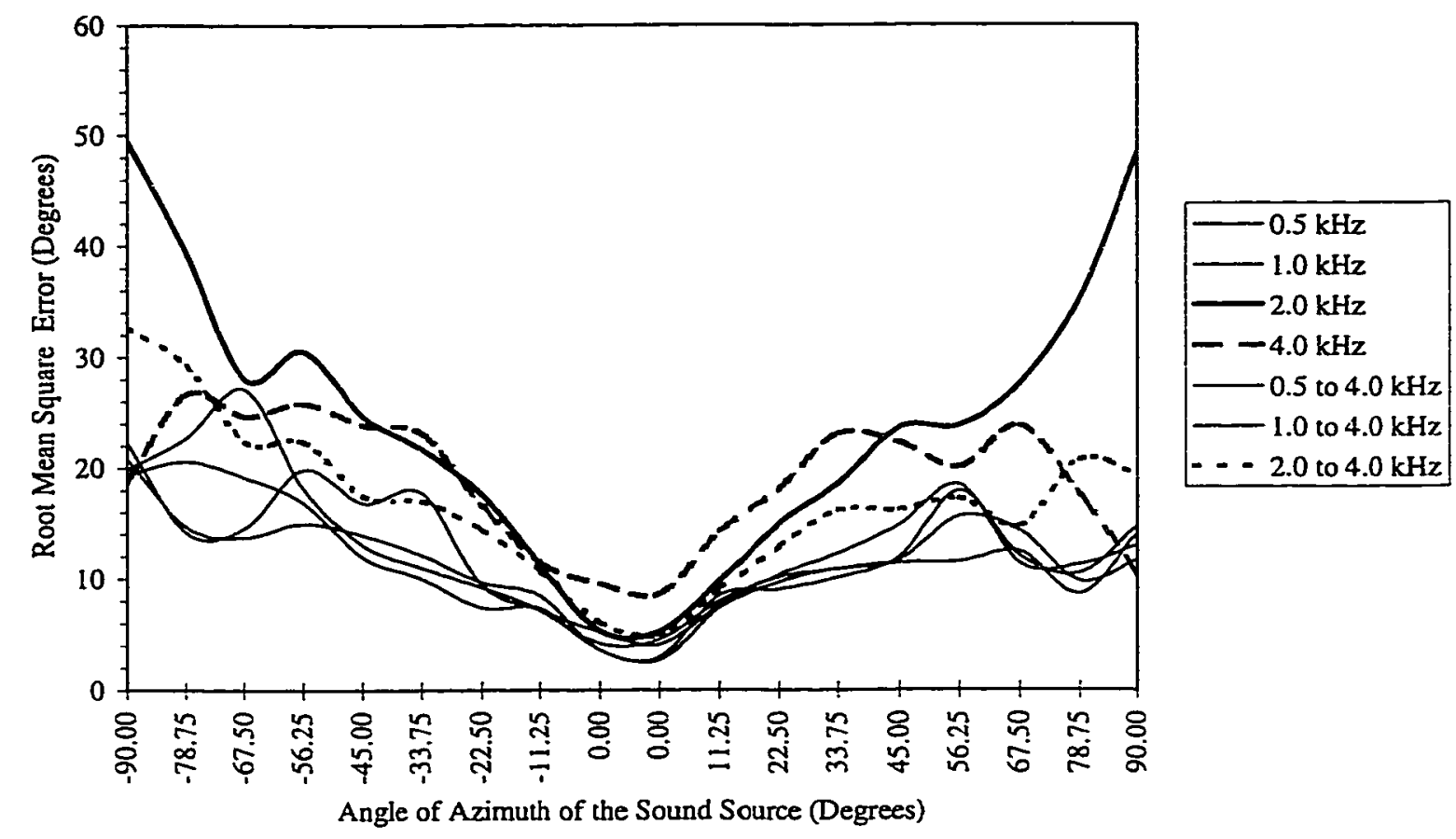

Figure 17: Effect of signal frequency and azimuth on root mean square error. For clarity of presentation, error bars are omitted.

\subsubsection{Azimuth and Interaural Intensity Difference Condition}

The interaction between the factors of azimuth and interaural intensity difference condition were also found to be significant for both the third-octave bands of noise and the band-pass noises. These results are presented in Figure 18 and Figure 19. 


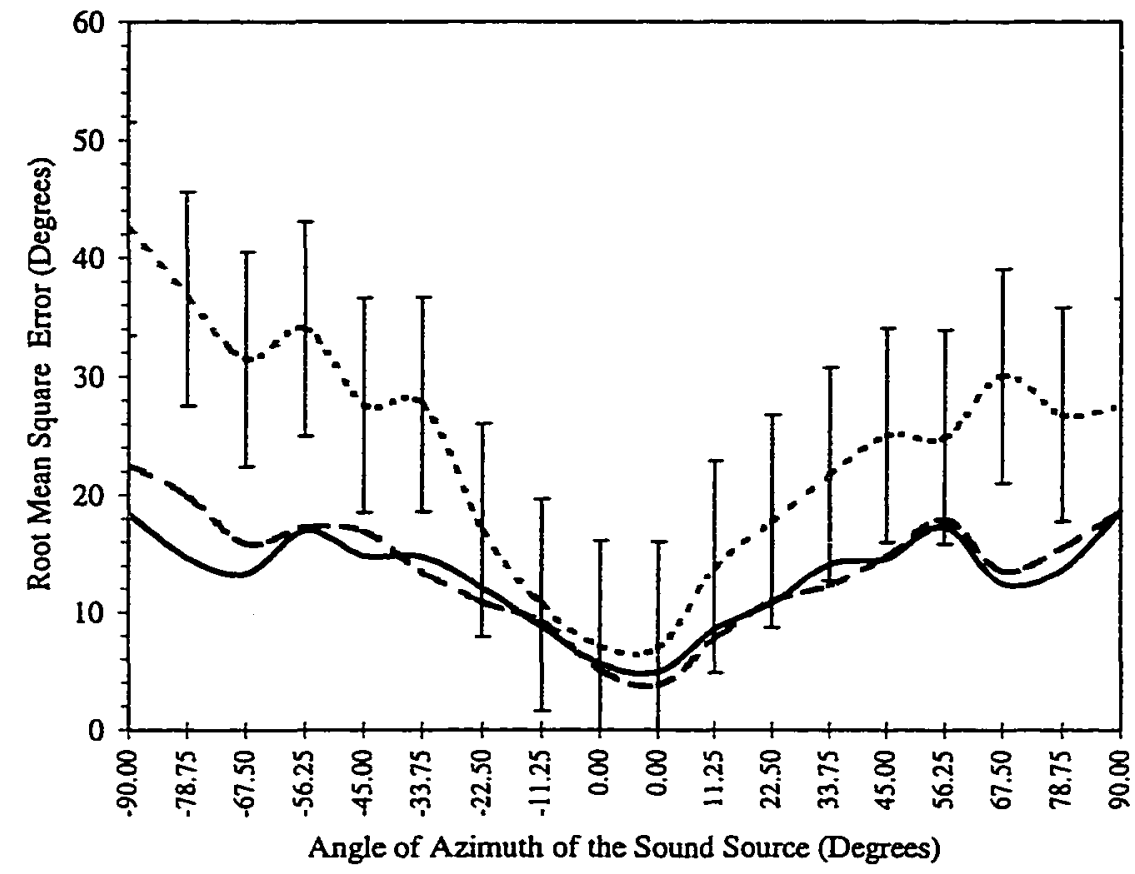

Figure 18: Effects of interaural intensity difference condition and azimuth on root mean square error for third-octave bands of noise. Error bars represent a difference at the 0.05 level of significance using Tukey's HSD. For clarity of presentation, error bars are shown only for the $100 \%$ IID attenuation condition.

Figure 18 displays the effect of interaural intensity difference condition upon root mean square error for third-octave bands across all azimuths that were tested. The plots for the unattenuated interaural intensity difference and 50\% attenuated interaural intensity difference are not different, but for the $100 \%$ attenuated condition, the magnitude of the errors rises rapidly with azimuth on both the left and right sides. This effect, however, is asymmetrical with a tendency toward greater error magnitudes on the left. Root mean square errors for the $100 \%$ attenuated condition become significantly greater from $33.75^{\circ}$ to $-90^{\circ}$ on the left, at $45^{\circ}$, and from $67.5^{\circ}$ to $90^{\circ}$ on the right. In contrast, the plots for the narrow bands of noise shown in Figure 19 are quite similar on the right side. On 
the left side, however, there is a dramatic increase in the magnitude of root mean square errors for the $100 \%$ attenuated condition at loudspeaker locations beyond $-45^{\circ}$.

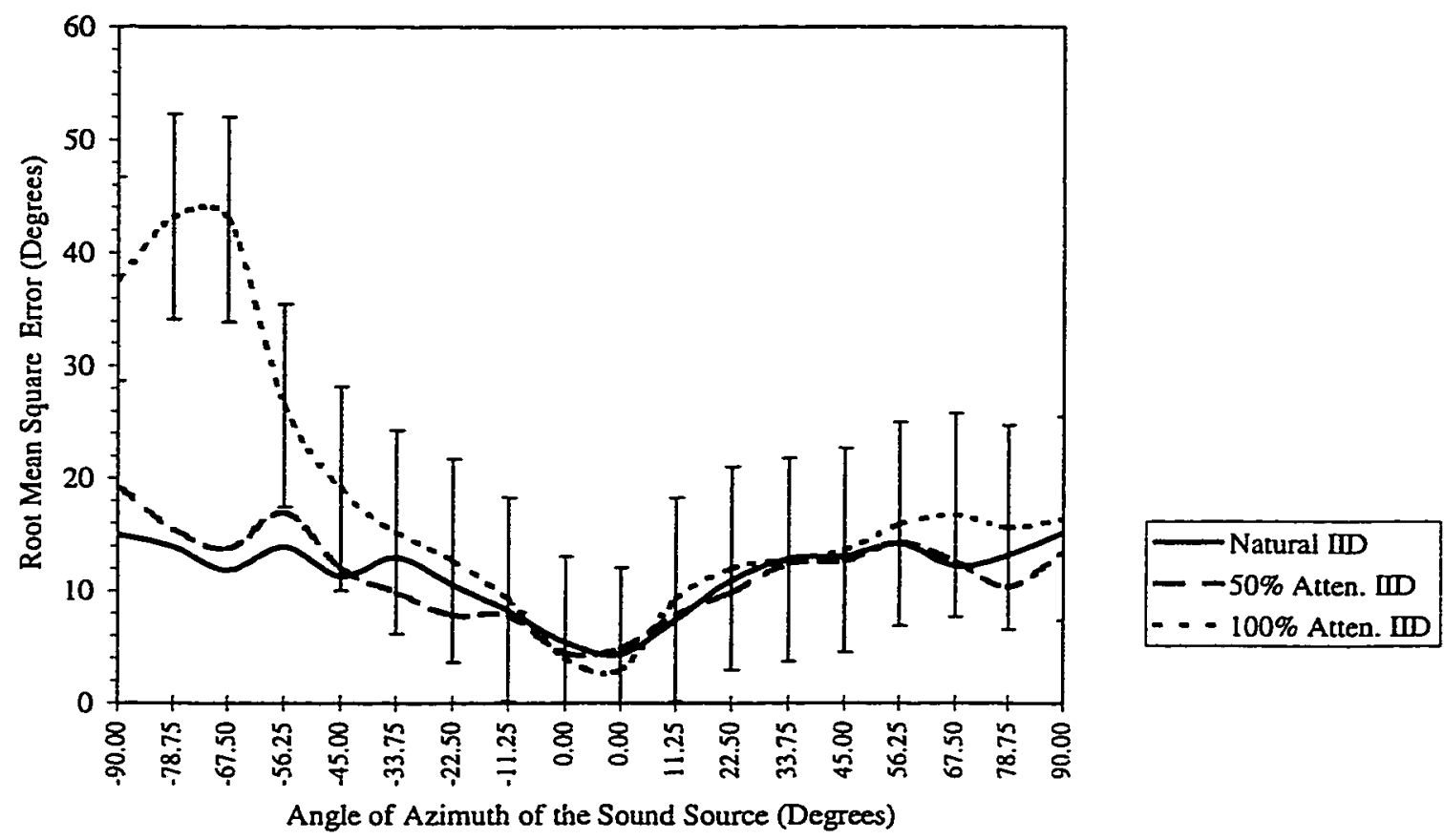

Figure 19: Effects of interaural intensity difference condition and azimuth on root mean square error for band-pass noises. Error bars represent a difference at the 0.05 level of significance using Tukey's HSD. For clarity of presentation, error bars are shown only for the $100 \%$ IID attenuation condition.

\subsubsection{Azimuth, Signal Frequency and Interaural Intensity Difference Condition}

A significant interaction was also observed among the factors azimuth, signal frequency and interaural intensity difference condition for both the third-octave bands and the band-pass noises $(p<0.01)$. The interaction is presented graphically in the Appendix in Figure 71 through Figure 77.

Figure 71 shows that there is no effect of interaural intensity difference condition for the $0.5 \mathrm{kHz}$ third-octave band. However, for the $1.0 \mathrm{kHz}$ third-octave band (Figure 72) there is a significant increase in root mean square error for the $100 \%$ attenuated 
condition at $-33.75^{\circ}$ and $-90^{\circ}$ to the left of center. For the $2.0 \mathrm{kHz}$ and $4.0 \mathrm{kHz}$ thirdoctave bands, displayed in Figure 73 and Figure 74, there is a great increase in root mean square error for the $100 \%$ attenuated condition. The magnitude of the error increases as the angle of azimuth increases to the left or right of the center. For the $4.0 \mathrm{kHz}$ thirdoctave band signal, the magnitude of the error for the $100 \%$ attenuated condition dropped at $90^{\circ}$ on the right. A similar but not significant effect at $-90^{\circ}$ is seen on the left.

For the band-pass noises, shown in Figure 75 through Figure 77, the negative effect of the $100 \%$ attenuated condition on localization accuracy occurred on the extreme left side (azimuths from $-56.50^{\circ}$ to $-78.50^{\circ}$ for 0.5 to $4.0 \mathrm{kHz} ;-67.50^{\circ}$ to $-90.00^{\circ}$ for 1.0 to $4.0 \mathrm{kHz}$ and 2.0 to $4.0 \mathrm{kHz}$ ).

\subsubsection{Room}

Recordings for this experiment were made in two rooms with very different characteristics: a sound-treated, anechoic test room and a large, windowless meeting room, called the minitheater.

There was no significant difference in root mean square error for signals recorded in the anechoic room versus those recorded in the minitheater, although there were some significant three- and four-way interactions involving the factor room.

\section{4 $\operatorname{Bias}\left(E_{b}\right)$}

\section{A General Linear Model Repeated Measures ANOVA (SPSS Advanced} Statistics $^{\text {TM }}$, 1996) was performed separately for each signal category (third-octave band and band-pass noises) over the dependent variable bias $\left(\boldsymbol{E}_{\boldsymbol{b}}\right)$. The factors for the analysis were interaural intensity difference condition (IID, 3 levels), signal type (FREQ, 4 levels for third-octave bands, 3 levels for band-pass noises), loudspeaker location (AZIM, 18 
levels ${ }^{3}$ ) and room (ROOM, 2 levels), with repeated measures over 5 subjects. The ANOVA tables for these analyses appear in Table 4 and Table 5.

${ }^{3}$ See footnote 2 on page 50. 
Table 4: ANOVA table of bias for third-octave bands of noise. IID represents interaural intensity difference condition, FREQ represents signal type, AZIM represents loudspeaker location and ROOM represents room type.

\begin{tabular}{|c|c|c|c|c|c|}
\hline \multicolumn{6}{|c|}{ Tests of Within-Subjects Effects } \\
\hline \multicolumn{6}{|c|}{ Bias - Third-octave Bands } \\
\hline & Sphericity & sumed & & & \\
\hline Source & $\begin{array}{l}\text { Type III } \\
\text { Sum of } \\
\text { Squares }\end{array}$ & $\mathrm{df}$ & $\begin{array}{l}\text { Mean } \\
\text { Square }\end{array}$ & $\bar{F}$ & Sig. \\
\hline $\mathrm{m}$ & 31.462 & 2 & 15.731 & 6.498 & 0.021 \\
\hline Error(iiD) & 19.368 & 8 & 2.421 & & \\
\hline FREQ & 1.833 & 3 & 0.611 & 0.048 & 0.985 \\
\hline Error(FREQ) & 151.703 & 12 & 12.642 & & \\
\hline$\overline{A Z I M}$ & 1460.796 & 17 & 85.929 & 19.273 & 0.000 \\
\hline ErTor(AZTM) & 303.187 & 68 & 4.459 & & \\
\hline ROOM & 6.144 & 1 & 6.144 & 2.312 & 0.203 \\
\hline Error(ROOM) & 10.629 & 4 & 2.657 & & \\
\hline III * FREQ & 8.054 & 6 & 1.342 & 0.984 & 0.458 \\
\hline Error(IID * FREQ) & 32.738 & 24 & 1.364 & & \\
\hline IID * AZIM & 1115.289 & 34 & 32.803 & 45.886 & 0.000 \\
\hline Error(IID*AZTM) & 97.224 & 136 & 0.715 & & \\
\hline III * ROOM & 9.350 & 2 & 4.675 & 3.861 & 0.067 \\
\hline Error(III * ROOM) & 9.687 & 8 & 1.211 & & \\
\hline FREQ * AZIM & 1043.035 & 51 & 20.452 & 14.445 & 0.000 \\
\hline Error(FREQ*AZMM) & 288.837 & 204 & 1.416 & & \\
\hline FREQ * ROOM & 6.461 & 3 & 2.154 & 0.511 & 0.683 \\
\hline Error(FREQ * ROOM) & 50.625 & 12 & 4.219 & & \\
\hline AZIM * ROOM & 43.687 & 17 & 2.570 & 3.623 & 0.000 \\
\hline Error(AZIM * ROOM) & 48.233 & 68 & 0.709 & & \\
\hline III * FREQ * AZIM & 865.394 & 102 & 8.484 & 16.159 & 0.000 \\
\hline Error(III * FREQ * AZIM) & 214.216 & 408 & 0.525 & & \\
\hline III * FREQ * ROOM & 19.922 & 6 & 3.320 & 4.458 & 0.004 \\
\hline Error(III * FREQ * ROOM) & 17.877 & 24 & 0.745 & & \\
\hline IID * AZIM * ROOM & 16.735 & 34 & 0.492 & 1.378 & 0.102 \\
\hline Error(IID * AZIM * ROOM) & 48.565 & 136 & 0.357 & & \\
\hline FREQ * AZIM * ROOM & 63.353 & 51 & 1.242 & 1.954 & 0.001 \\
\hline Error(FREQ * AZIM * ROOM) & 129.695 & 204 & 0.636 & & \\
\hline III * FREQ * AZIM * ROOM & 61.452 & 102 & 0.602 & 1.646 & 0.000 \\
\hline $\begin{array}{l}\text { Error(IID * FREQ * AZIM * } \\
\text { ROOM) }\end{array}$ & 149.306 & 408 & 0.366 & & \\
\hline
\end{tabular}


Table 5: ANOVA table of bias for band-pass noises. IID represents interaural intensity difference condition, FREQ represents signal type, AZIM represents loudspeaker location and ROOM represents room type.

\begin{tabular}{|c|c|c|c|c|c|}
\hline \multicolumn{6}{|c|}{ Tests of Within-Subjects Effects } \\
\hline \multicolumn{6}{|c|}{ Bias - Band-pass Noises } \\
\hline & Sphericity & ssumed & & & \\
\hline Source & $\begin{array}{l}\text { Type III } \\
\text { Sum of } \\
\text { Squares }\end{array}$ & $\mathrm{df}$ & $\begin{array}{l}\text { Mean } \\
\text { Square }\end{array}$ & $\mathrm{F}$ & Sig. \\
\hline Ii⿱一⿻上丨丨 & 121.711 & 2 & 60.855 & 90.407 & 0.000 \\
\hline Error(IID) & 5.385 & 8 & 0.673 & & \\
\hline FREQ & 5.225 & 2 & 2.613 & 0.536 & 0.605 \\
\hline Error(FREQ) & 39.021 & 8 & 4.878 & & \\
\hline AZIM & 724.425 & 17 & 42.613 & 12.010 & 0.000 \\
\hline Error(AZIM) & 241.276 & 68 & 3.548 & & \\
\hline ROOM & 0.046 & 1 & 0.046 & 0.052 & 0.830 \\
\hline Error(ROOM) & 3.485 & 4 & 0.871 & & \\
\hline III * FREQ & 2.555 & 4 & 0.639 & 0.255 & 0.902 \\
\hline Error(IID * FREQ) & 40.112 & 16 & 2.507 & & \\
\hline III * AZIM & 517.647 & 34 & 15.225 & 15.831 & 0.000 \\
\hline Error(III*AZIM) & 130.795 & 136 & 0.962 & & \\
\hline II * ROOM & 9.266 & 2 & 4.633 & 2.509 & 0.143 \\
\hline Error(IID * ROOM) & 14.774 & 8 & 1.847 & & \\
\hline FREQ * AZIM & 103.906 & 34 & 3.056 & 2.826 & 0.000 \\
\hline Error(FREQ*AZIM) & 147.079 & 136 & 1.081 & & \\
\hline FREQ * ROOM & 0.191 & 2 & 0.096 & 0.224 & 0.804 \\
\hline Error(FREQ * ROOM) & 3.408 & 8 & 0.426 & & \\
\hline AZIM * ROOM & 30.421 & 17 & 1.789 & 2.819 & 0.001 \\
\hline Error(AZIM * ROOM) & 43.158 & 68 & 0.635 & & \\
\hline IID * FREQ * AZIM & 82.178 & 68 & 1.209 & 2.122 & 0.000 \\
\hline Error(III * FREQ * AZIM) & 154.924 & 272 & 0.570 & & \\
\hline III * FREQ * ROOM & 5.158 & 4 & 1.290 & 1.744 & 0.190 \\
\hline Error(III * FREQ * ROOM) & 11.832 & 16 & 0.739 & & \\
\hline II * AZIM * ROOM & 16.125 & 34 & 0.474 & 1.319 & 0.136 \\
\hline Error(III * AZIM * ROOM) & 48.893 & 136 & 0.360 & & \\
\hline FREQ * AZIM * ROOM & 17.267 & 34 & 0.508 & 1.684 & 0.019 \\
\hline Error(FREQ * AZIM * ROOM) & 41.006 & 136 & 0.302 & & \\
\hline III * FREQ * AZIM * ROOM & 30.315 & 68 & 0.446 & 1.838 & 0.000 \\
\hline $\begin{array}{l}\text { Error(IID * FREQ * AZTM * } \\
\text { ROOM) }\end{array}$ & 65.958 & 272 & 0.242 & & \\
\hline
\end{tabular}


There was no significant main effect for the factor room or signal frequency in either analysis, nor was their a significant interaction between signal frequency and interaural intensity difference condition, indicating that the attenuation of interaural intensity differences did not affect different frequencies differently on this measure.

\subsection{Interaural Intensity Difference Condition}

The main effect of interaural intensity difference condition was significant for both the third-octave band noises and the band-pass noises. These results are presented graphically in Figure 20.

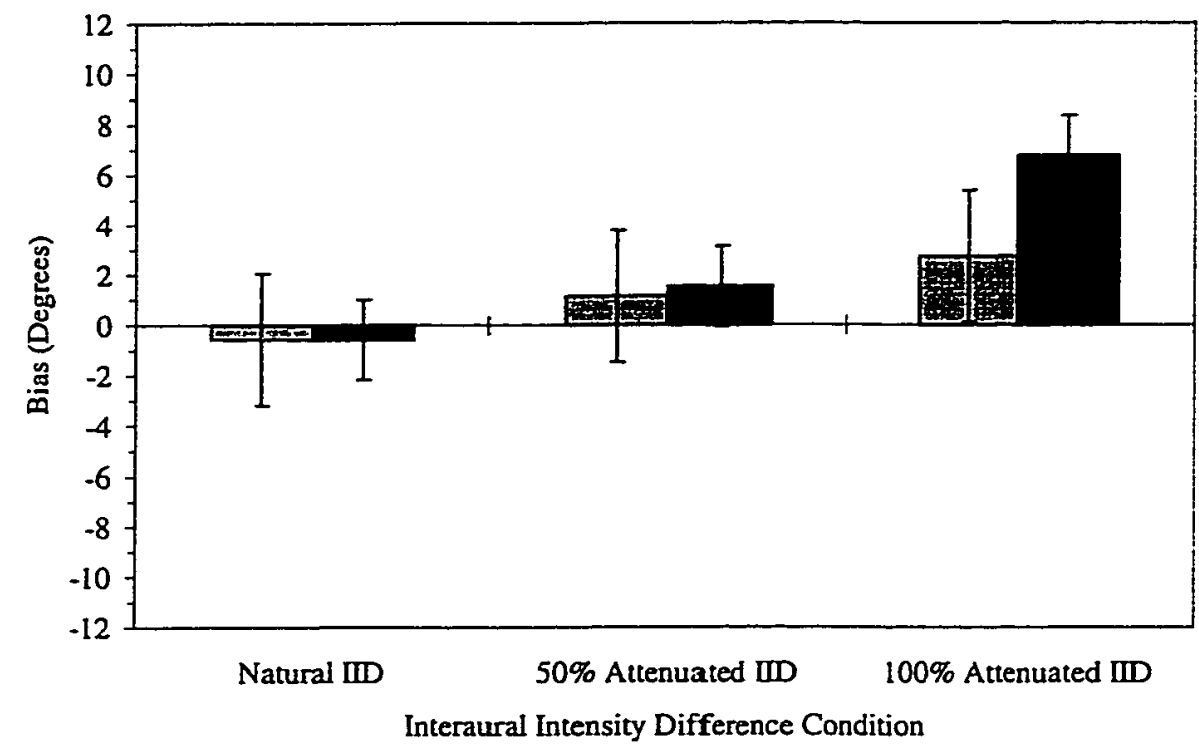

QThird-octave bands

Band-pass noises

Figure 20: Effect of interaural intensity difference condition on bias. Error bars represent a difference at the $\mathbf{0 . 0 5}$ level of significance using Tukey's HSD.

The lighter colored bars in Figure 20 show the effect of interaural intensity difference condition upon bias for third-octave bands of noise. The biases are small, but there is a tendency for the responses to be more positive (biased toward the right) as 
interaural intensity differences are reduced or eliminated. As indicated by the error bars, post hoc testing (Tukey HSD) revealed that the bias for the $100 \%$ attenuated condition was significantly greater than that for the unattenuated condition, but not different from that of the $50 \%$ attenuated condition. Nor was there a difference between the bias for the $50 \%$ attenuated condition relative to the unattenuated condition.

Differences in bias were greater for the band-pass noises than they were for the third-octave band noises. This is shown by the darker colored bars in Figure 20. The $100 \%$ attenuated condition had a significantly greater positive (toward the right) bias ( $\mathrm{p}<$ 0.01 ) relative to unattenuated and $50 \%$ attenuated. The bias for the $50 \%$ attenuated condition was significantly different from the unattenuated condition $(p<0.05)$, but the difference was small ( -0.05 vs. 0.14$)$. Nevertheless, for these subjects, reducing or eliminating the interaural intensity difference appears to have had the effect of biasing localization errors toward the right.

\subsubsection{Azimuth}

The effect of sound source azimuth was found to be significant for both the thirdoctave band noises and the band-pass noises $(p<0.01)$. Errors were biased toward the right for azimuths to the left and toward the left for azimuths to the right for the most extreme azimuths $\left( \pm 90^{\circ}\right)$. This effect can be observed in Figure 21 for both signal types and rooms.

\subsubsection{Azimuth and Room}

There was a significant interaction between room and azimuth for both noise types $(\mathrm{p}<0.01)$. This interaction is shown in Figure 21. 


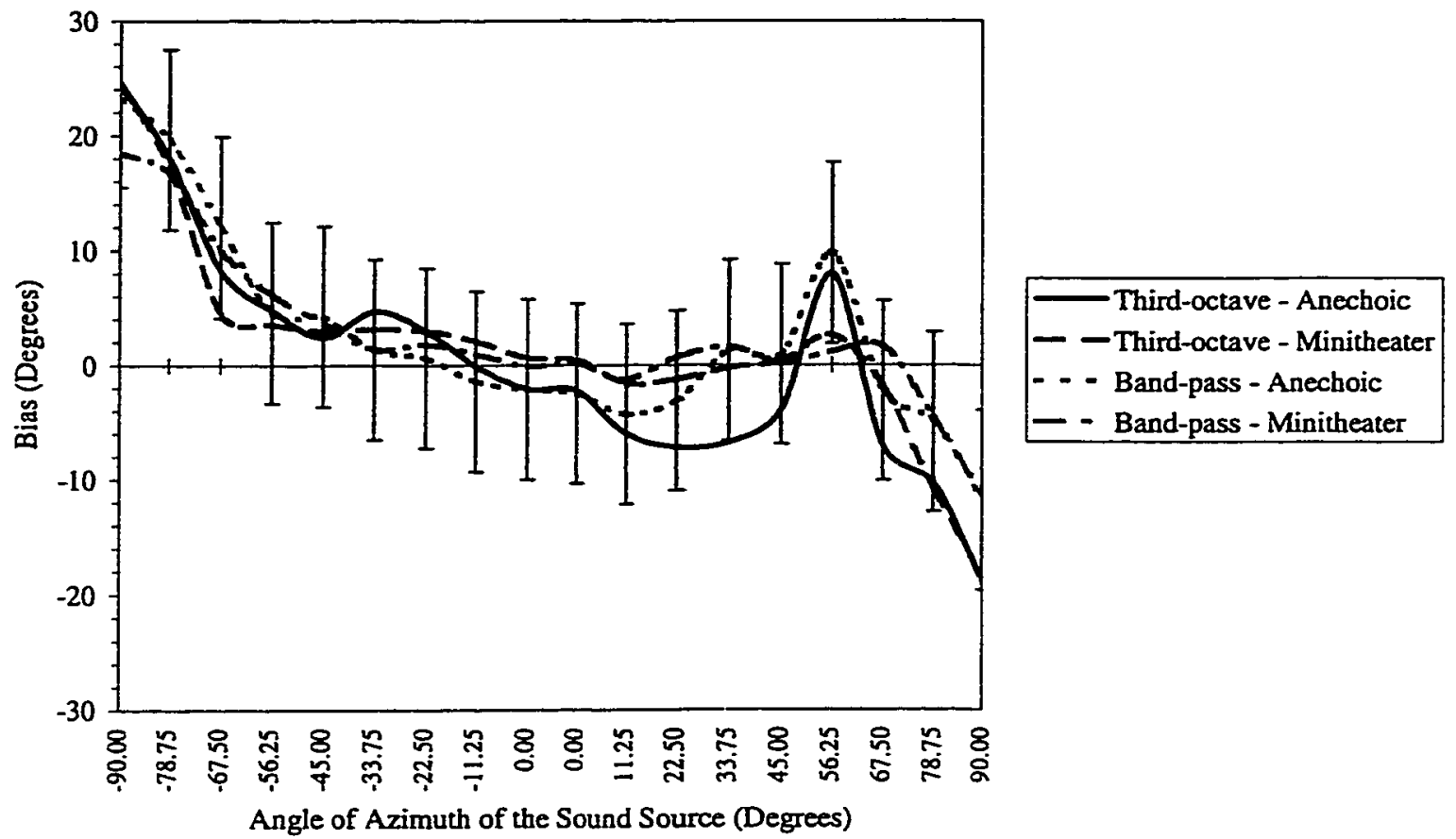

Figure 21: Effect of room and sound source azimuth on bias for band-pass noises. Error bars represent a difference at the 0.05 level of significance using Tukey's HSD. For clarity of presentation, error bars are displayed only for the band-pass noises in the anechoic room.

The interaction appears to be based upon the fact that there is a bump in the error function at $56.25^{\circ}$ to the right for the anechoic room only. The effect is significant only for the band-pass noises.

\subsubsection{Azimuth and Signal Frequency}

A significant interaction was observed between azimuth and signal frequency for both noise types $(\mathrm{p}<0.01)$. This interaction is displayed in Figure 22 and Figure 23.

For the third-octave bands, shown in Figure 22, the interaction consists of a bias to the right on the left side and bias to the left on the right side for the higher frequency signals ( 2.0 and $4.0 \mathrm{kHz}$ ) relative to the lower frequency signals $(0.5$ and $1.0 \mathrm{kHz})$. This 
effect is significant for $2.0 \mathrm{kHz}$ at azimuths greater than $-67.50^{\circ}$ to the left and $+78.75^{\circ}$ to the right.

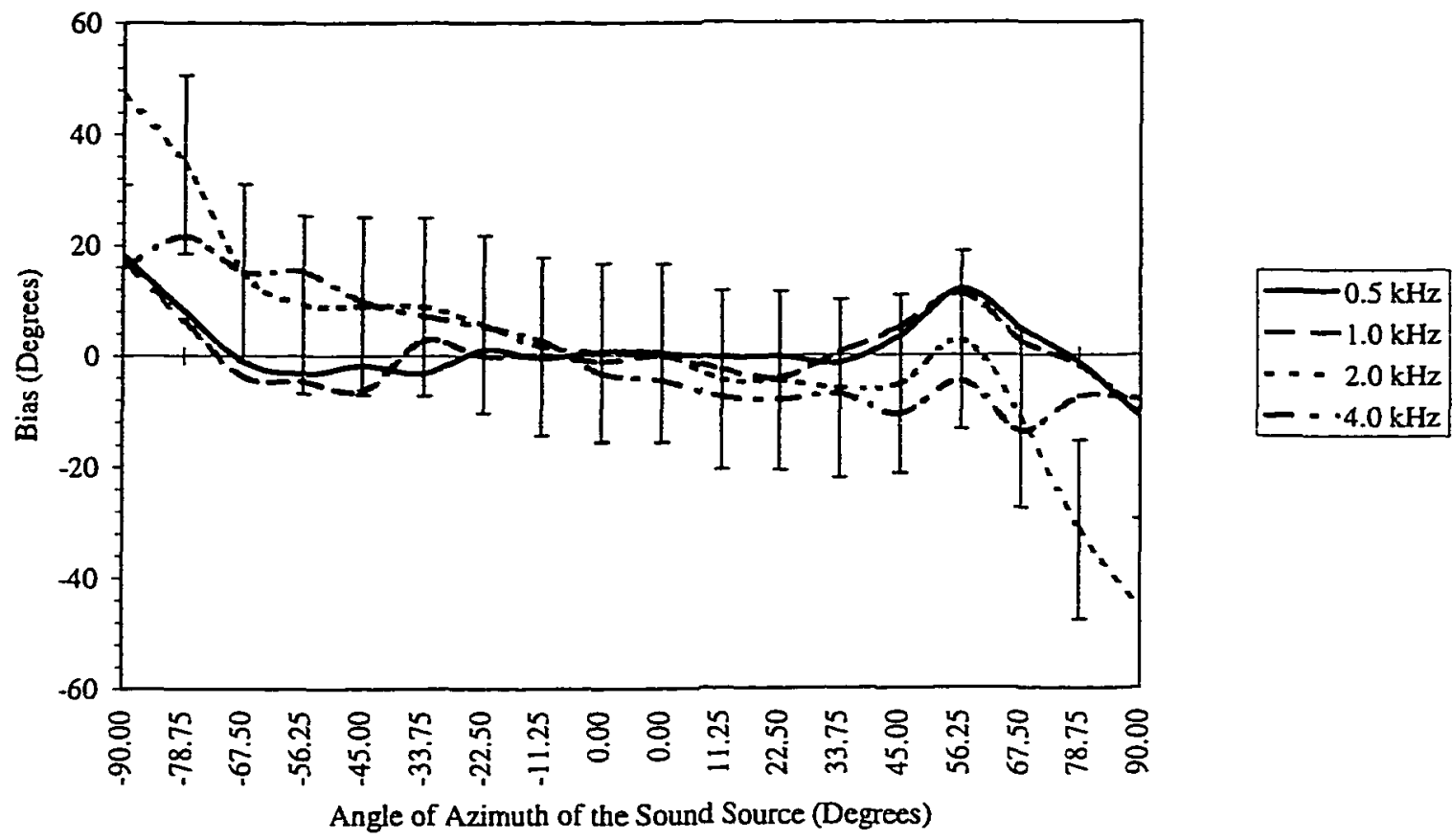

Figure 22: Effect of azimuth and signal frequency on bias for third-octave bands of noise. Error bars represent a difference at the 0.05 level of significance using Tukey's HSD. For clarity of presentation, error bars are displayed only for the 4.0 kHz signal.

For the band-pass noises, shown in Figure 23, there is a significant bias toward the right at $-90^{\circ}$ for the 2.0 to $4.0 \mathrm{kHz}$ band-pass noise errors. 


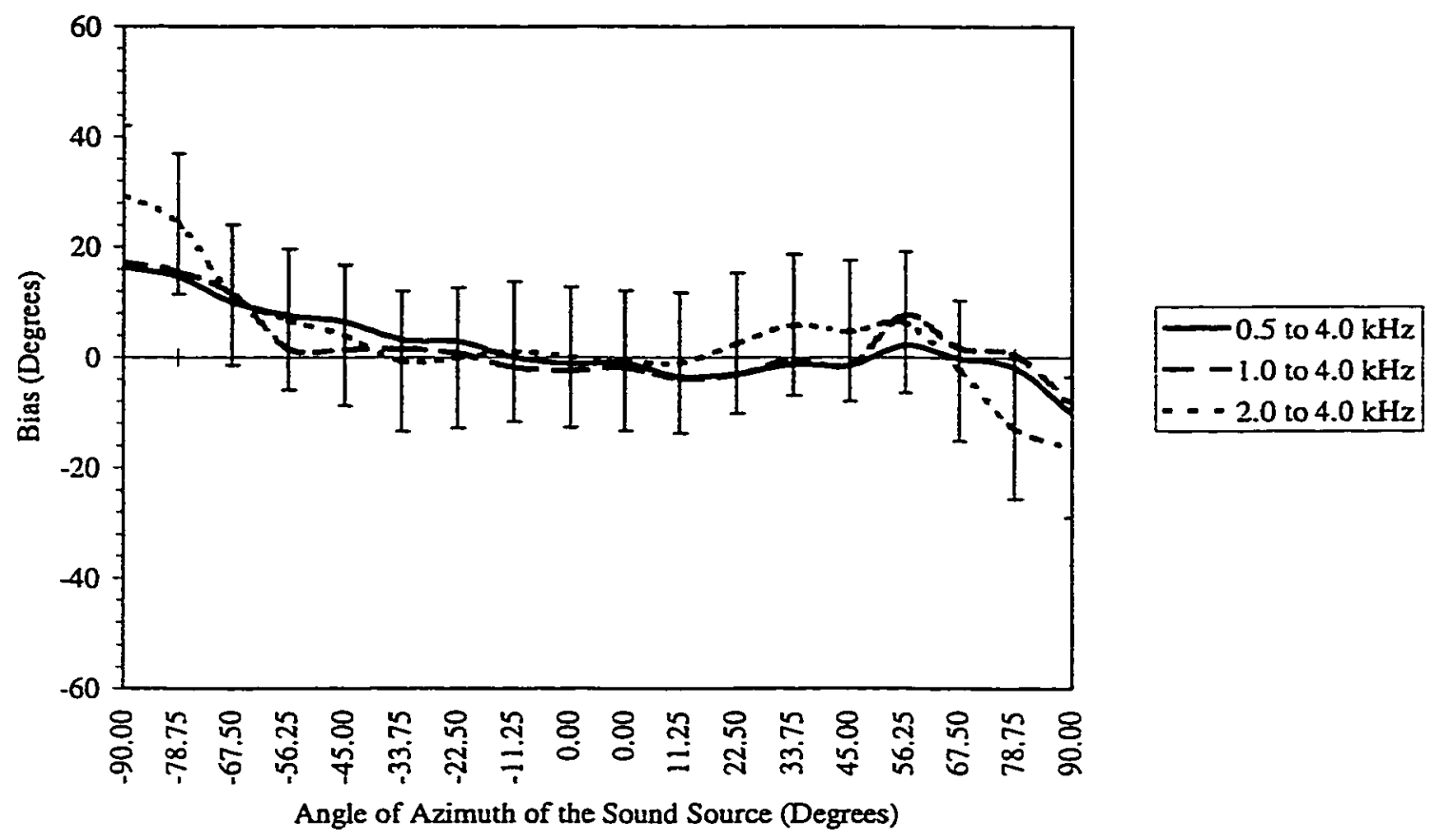

Figure 23: Effect of azimuth and signal frequency on bias for band-pass noises. Error bars represent a difference at the 0.05 level of significance using Tukey's HSD. For clarity of presentation, error bars are displayed only for the 2.0 to $4.0 \mathrm{kHz}$ signal.

\subsubsection{Azimuth and Interaural Intensity Difference Condition}

The interaction between azimuth and interaural intensity difference condition was significant for both noise types $(\mathrm{p}<0.01)$. Figure 24 and Figure 25 show this relationship for the third-octave bands and band-pass noises, respectively.

The most striking observation about the plots for the third-octave band noises in Figure 24 is the fact that bias for the $100 \%$ attenuated condition increases more rapidly in a positive direction as the angle of azimuth moves farther to the left of center and in a negative direction as the angle of azimuth moves to the right of center, relative to the unattenuated or $50 \%$ attenuated conditions. A similar observation may be made for the 
band-pass noises in Figure 25 except that the effect is asymmetrical and seen only on the left side from $-45.00^{\circ}$ to $-90.00^{\circ}$.

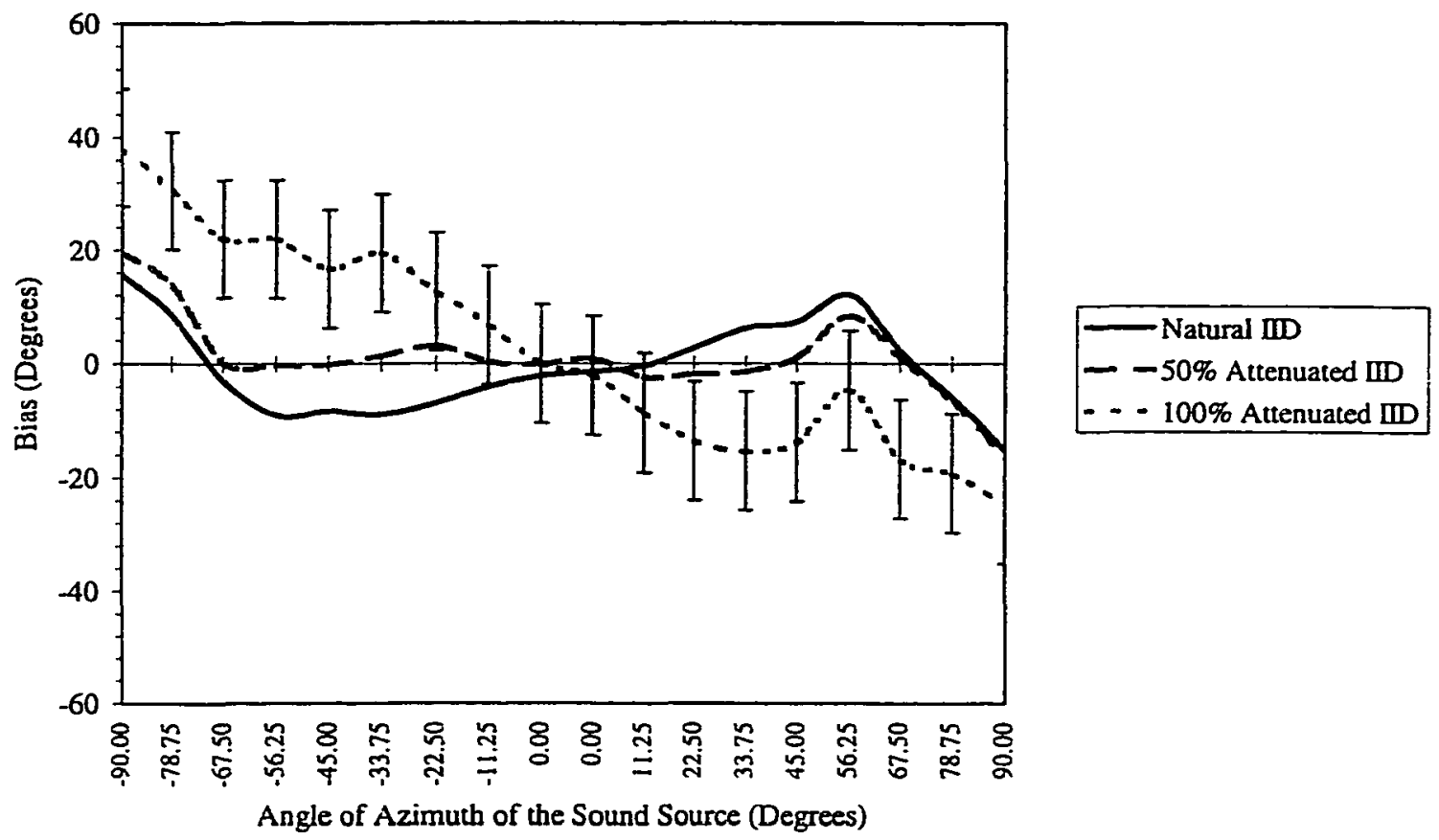

Figure 24: Effect of azimuth and interaural intensity difference condition on bias for third-octave bands of noise. Error bars represent a difference at the 0.05 level of significance using Tukey's HSD. For clarity of presentation, error bars are displayed only for the $\mathbf{1 0 0 \%}$ attenuated interaural intensity difference condition. 


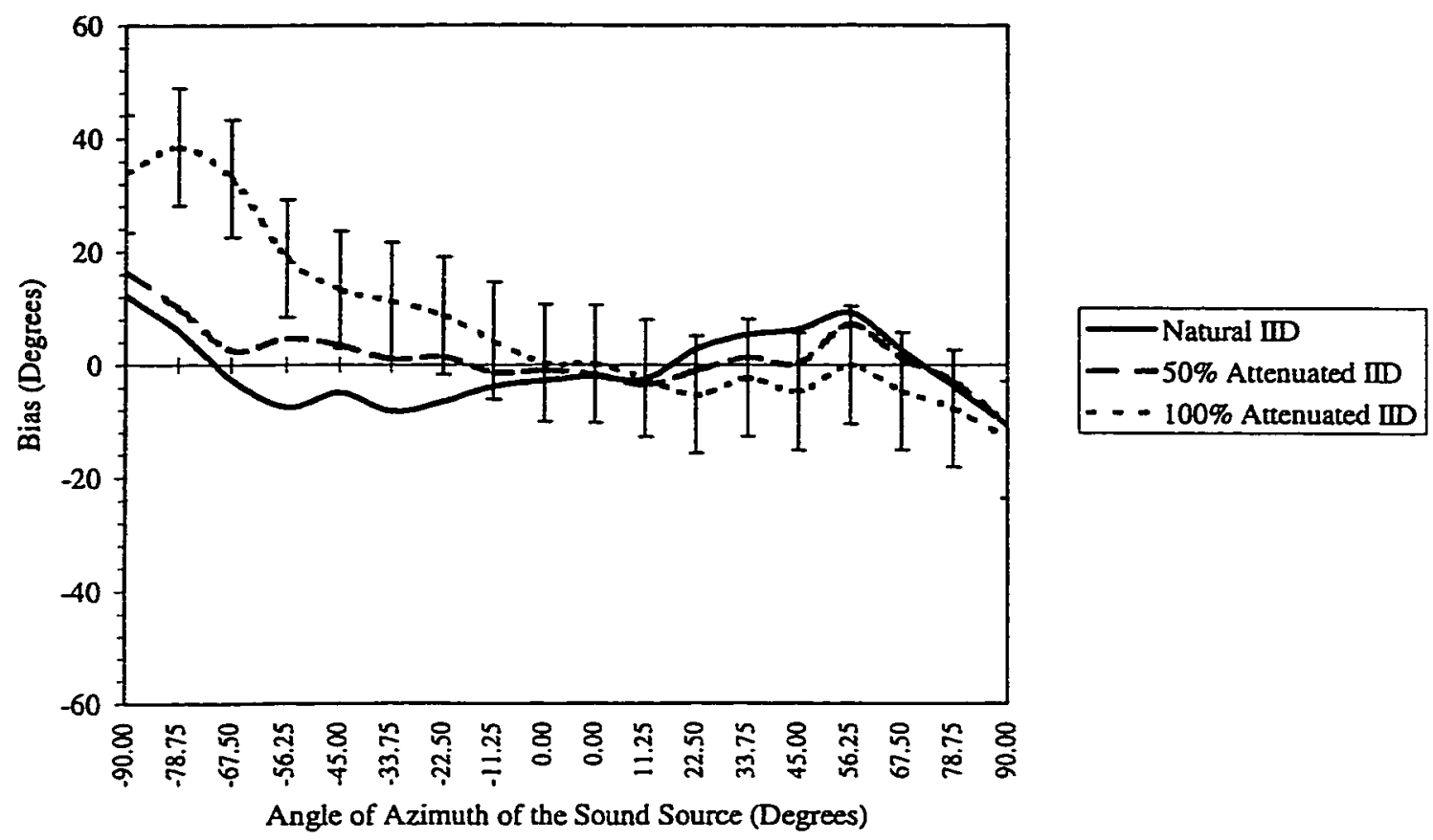

Figure 25: Effect of azimuth and interaural intensity difference condition on bias for band-pass noises. Error bars represent a difference at the 0.05 level of significance using Tukey's HSD. For clarity of presentation, error bars are displayed only for the $100 \%$ attenuated interaural intensity difference condition.

\subsubsection{Azimuth, Interaural Intensity Difference Condition and Frequency}

Analysis of the effect of interaural intensity difference condition and azimuth over the additional factor of frequency reveals that the observations made above for thirdoctave band noises are largely due to the effects of interaural intensity difference condition and azimuth on the higher frequency signals $(2.0 \mathrm{kHz}$ and $4.0 \mathrm{kHz}$ third-octave band noises). This relationship is illustrated in a series of figures presented in the Appendix (Figure 78 through Figure 81).

On the other hand, the interaction observed for the band-pass noises appears more consistent across frequency, but with increases in the magnitude of the effect as lowfrequency content decreases. This may be seen in Figure 82 through Figure 84, in the 
Appendix. Note the asymmetry of the effect. The bias toward the right for the $100 \%$ attenuated condition occurs on the left side with no significant bias on the right side.

Other interactions that were found to be significant in the analysis of the bias for the third-octave bands involved room by signal frequency by interaural intensity difference condition, room by signal frequency by azimuth, signal frequency by interaural intensity difference condition by azimuth and the four-way interaction room by signal frequency by interaural intensity difference condition by azimuth. For the bandpass noises, significant interactions were the same as those for the third-octave bands except for room by signal frequency by interaural intensity difference condition which was not significant. Examination of these interactions did not shed any further light on the effects of interaural intensity difference attenuation upon the localization of sound sources. The interactions are based largely upon the fact that for different azimuths, the effects of signal frequency and interaural intensity difference condition upon the mean localization score were different, but the differences were small and the patterns of difference were complex.

\subsection{Test-retest standard deviation $\left(\sigma_{E}\right)$ : Precision of Localization}

A General Linear Model Repeated Measures ANOVA (SPSS Advanced Statistics ${ }^{\mathrm{TM}}$, 1996) was performed separately for each signal category over the dependent variable test-retest standard deviation $\left(\sigma_{E}\right)$. The factors for the analysis were interaural intensity difference condition (IID, 3 levels), signal type (FREQ, 4 levels for third-octave bands, 3 levels for band-pass noises), loudspeaker location (AZIM, 18 levels ${ }^{4}$ ) and room

\footnotetext{
${ }^{4}$ See footnote 2 on page 50.
} 
(ROOM, 2 levels), with repeated measures over 5 subjects. The ANOVA tables for these analyses appear in Table 6 and Table 7. 
Table 6: ANOVA table of the test-retest standard deviation for third-octave bands of noise. IDD represents interaural intensity difference condition, FREQ represents signal type, AZIM represents loudspeaker location and ROOM represents room type.

\begin{tabular}{|c|c|c|c|c|c|}
\hline \multicolumn{6}{|c|}{ Tests of Within-Subjects Effects } \\
\hline \multicolumn{6}{|c|}{ Test-retest standard deviation - Third-octave Bands } \\
\hline \multicolumn{6}{|c|}{ Sphericity Assumed } \\
\hline Source & $\begin{array}{l}\text { Type III } \\
\text { Sum of } \\
\text { Squares }\end{array}$ & df & $\begin{array}{l}\text { Mean } \\
\text { Square }\end{array}$ & $\mathbf{F}$ & Sig. \\
\hline IID & 9.407 & 2 & 4.704 & 2.394 & 0.153 \\
\hline Error(ID) & 15.720 & 8 & 1.965 & & \\
\hline FREQ & 13.516 & 3 & 4.505 & 2.905 & 0.078 \\
\hline Error(FREQ) & 18.610 & 12 & 1.551 & & \\
\hline AZIM & 109.892 & 17 & 6.464 & 9.054 & 0.000 \\
\hline Error(AZIM) & 48.550 & 68 & 0.714 & & \\
\hline ROOM & 10.467 & 1 & 10.467 & 6.146 & 0.068 \\
\hline Error(ROOM) & 6.813 & 4 & 1.703 & & \\
\hline IID * FREQ & 6.898 & 6 & 1.150 & 1.182 & 0.349 \\
\hline Error(III * FREQ) & 23.341 & 24 & 0.973 & & \\
\hline III * AZIM & 24.434 & 34 & 0.719 & 1.812 & 0.009 \\
\hline Error(mD*AZIM) & 53.931 & 136 & 0.397 & & \\
\hline IID * ROOM & 2.695 & 2 & 1.348 & 2.750 & 0.123 \\
\hline Error(WD * ROOM) & 3.920 & 8 & 0.490 & & \\
\hline FREQ * AZIM & 34.920 & 51 & 0.685 & 1.420 & 0.047 \\
\hline Error(FREQ*AZTM) & 98.365 & 204 & 0.482 & & \\
\hline FREQ * ROOM & 1.510 & 3 & 0.503 & 0.352 & 0.788 \\
\hline Error(FREQ * ROOM) & 17.148 & 12 & 1.429 & & \\
\hline $\mathrm{AZMM} * \mathrm{ROOM}$ & 7.249 & 17 & 0.426 & 1.084 & 0.387 \\
\hline Error(AZIM * ROOM) & 26.757 & 68 & 0.393 & & \\
\hline II * FREQ * AZIM & 64.536 & 102 & 0.633 & 1.944 & 0.000 \\
\hline Error(ID * FREQ * AZIM) & 132.795 & 408 & 0.325 & & \\
\hline IID * FREQ * ROOM & 4.370 & 6 & 0.728 & 0.863 & 0.536 \\
\hline Error(IID * FREQ * ROOM) & 20.255 & 24 & 0.844 & & \\
\hline IID * AZIM * ROOM & 10.072 & 34 & 0.296 & 0.909 & 0.615 \\
\hline Error(IID * AZIM * ROOM) & 44.309 & 136 & 0.326 & & \\
\hline FREQ $*$ AZIM * ROOM & 15.364 & 51 & 0.301 & 0.749 & 0.889 \\
\hline Error(FREQ * AZIM * ROOM) & 82.028 & 204 & 0.402 & & \\
\hline IID * FREQ * AZIM * ROOM & 28.496 & 102 & 0.279 & 0.786 & 0.929 \\
\hline $\begin{array}{l}\text { Error(ID * FREQ * AZIM * } \\
\text { ROOM) }\end{array}$ & 145.039 & 408 & 0.355 & & \\
\hline
\end{tabular}


Table 7: ANOVA table of the test-retest standard deviation for band-pass noises. IID represents interaural intensity difference condition, FREQ represents signal type, AZIM represents loudspeaker location and ROOM represents room type.

\begin{tabular}{|c|c|c|c|c|c|}
\hline \multicolumn{6}{|c|}{ Tests of Within-Subjects Effects } \\
\hline \multicolumn{6}{|c|}{ Test-retest Standard Deviation - Band-pass Noises } \\
\hline \multicolumn{6}{|c|}{ Sphericity Assumed } \\
\hline Source & $\begin{array}{l}\text { Type WI } \\
\text { Sum of } \\
\text { Squares }\end{array}$ & df & $\begin{array}{l}\text { Mean } \\
\text { Square }\end{array}$ & $\bar{F}$ & Sig. \\
\hline III & 7.794 & 2 & 3.897 & 7.784 & 0.013 \\
\hline Error(IID) & 4.005 & 8 & 0.501 & & \\
\hline FREQ & 10.163 & 2 & 5.082 & 5.011 & 0.039 \\
\hline Error(FREQ) & 8.112 & 8 & 1.014 & & \\
\hline AZIM & 83.331 & 17 & 4.902 & 6.875 & 0.000 \\
\hline Error(AZIM) & 48.483 & 68 & 0.713 & & \\
\hline ROOM & 0.398 & 1 & 0.398 & 1.165 & 0.341 \\
\hline Error(ROOM) & 1.366 & 4 & 0.341 & & \\
\hline II * FREQ & 1.861 & 4 & 0.465 & 1.323 & 0.304 \\
\hline Error(IID * FREQ) & 5.628 & 16 & 0.352 & & \\
\hline $\mathrm{DD} * \mathrm{AZMM}$ & 24.012 & 34 & 0.706 & 2.487 & 0.000 \\
\hline Error(IID*AZIM) & 38.617 & 136 & 0.284 & & \\
\hline IID * ROOM & 0.130 & 2 & 0.065 & 0.245 & 0.789 \\
\hline Error(ID * ROOM) & 2.122 & 8 & 0.265 & & \\
\hline FREQ * AZMM & 15.195 & 34 & 0.447 & 1.777 & 0.011 \\
\hline Error(FREQ*AZIM) & 34.203 & 136 & 0.251 & & \\
\hline FREQ * ROOM & 0.798 & 2 & 0.399 & 0.663 & 0.542 \\
\hline Error(FREQ * ROOM) & 4.815 & 8 & 0.602 & & \\
\hline $\mathrm{AZIM} * \mathrm{ROOM}$ & 6.427 & 17 & 0.378 & 1.633 & 0.080 \\
\hline Error(AZIM * ROOM) & 15.743 & 68 & 0.232 & & \\
\hline $\mathrm{IID}$ * FREQ * AZMM & 17.740 & 68 & 0.261 & 1.261 & 0.102 \\
\hline Error(ID * FREQ * AZIM) & 56.288 & 272 & 0.207 & & \\
\hline IID * FREQ * ROOM & 1.340 & 4 & 0.335 & 1.167 & 0.362 \\
\hline Error(II * FREQ * ROOM) & 4.594 & 16 & 0.287 & & \\
\hline II * AZIM * ROOM & 5.802 & 34 & 0.171 & 0.773 & 0.807 \\
\hline Error(IID * AZIM * ROOM) & 30.018 & 136 & 0.221 & & \\
\hline FREQ * AZIM * ROOM & 8.373 & 34 & 0.246 & 1.206 & 0.225 \\
\hline Error(FREQ * AZIM * ROOM) & 27.777 & 136 & 0.204 & & \\
\hline IID * FREQ * AZIM * ROOM & 17.943 & 68 & 0.264 & 1.233 & 0.125 \\
\hline $\begin{array}{l}\text { Error(ID * FREQ * AZIM * } \\
\text { ROOM) }\end{array}$ & 58.198 & 272 & 0.214 & & \\
\hline
\end{tabular}




\subsubsection{Room}

The factor room was non-significant for both signal categories.

\subsubsection{Signal Frequency}

The factor signal frequency was non-significant for the third-octave bands, but significant for the band-pass signals $(\mathrm{p}<0.05)$. In general, $\sigma_{E}$ increased with decreasing low-frequency content for the band-pass signals. A post-hoc analysis (Tukey HSD) indicated that $\sigma_{E}$ for the 2.0 to $4.0 \mathrm{kHz}$ band-pass signal was significantly greater than that for the 0.5 to $4.0 \mathrm{kHz}$ signal, but not different from that for the 1.0 to $4.0 \mathrm{kHz}$ signal. These results are plotted Figure 26.

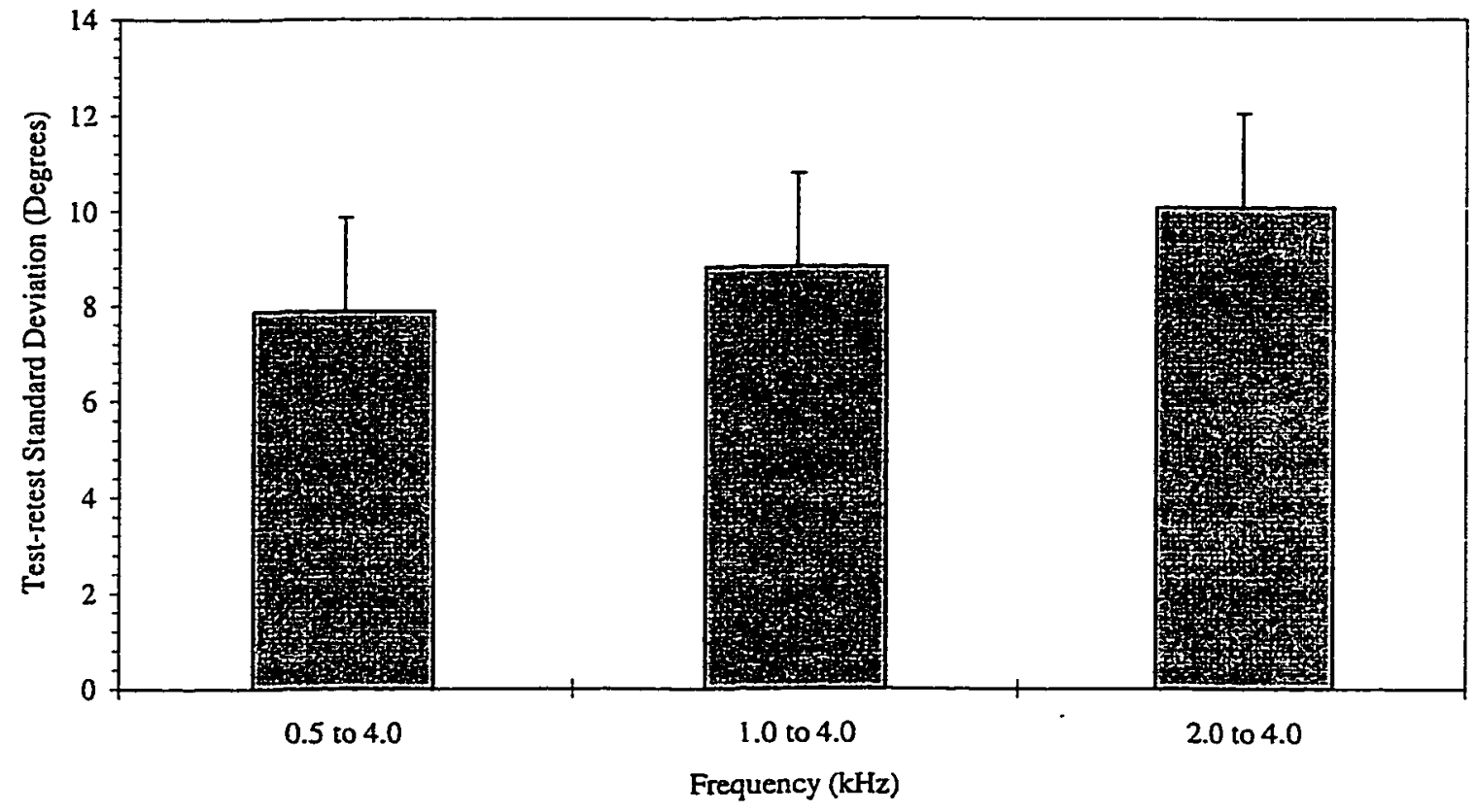

Figure 26: Effect of signal frequency on test-retest standard deviation for band-pass noises. Error bars represent a difference at the 0.05 level of significance using Tukey's HSD. 


\subsubsection{Interaural Intensity Difference Condition}

Like signal frequency, the effect of the factor interaural intensity difference condition was not significant for the third-octave bands, but was significant $(p<0.05)$ for the band-pass noises. The $100 \%$ attenuated condition had a significantly greater value for $\sigma_{E}$ than the unattenuated or $50 \%$ attenuated conditions. This effect was similar for all of the band-pass signals: there was no significant interaction between interaural intensity difference condition and signal frequency. The effect of interaural intensity difference condition for the band-pass noises is shown graphically in Figure 27.

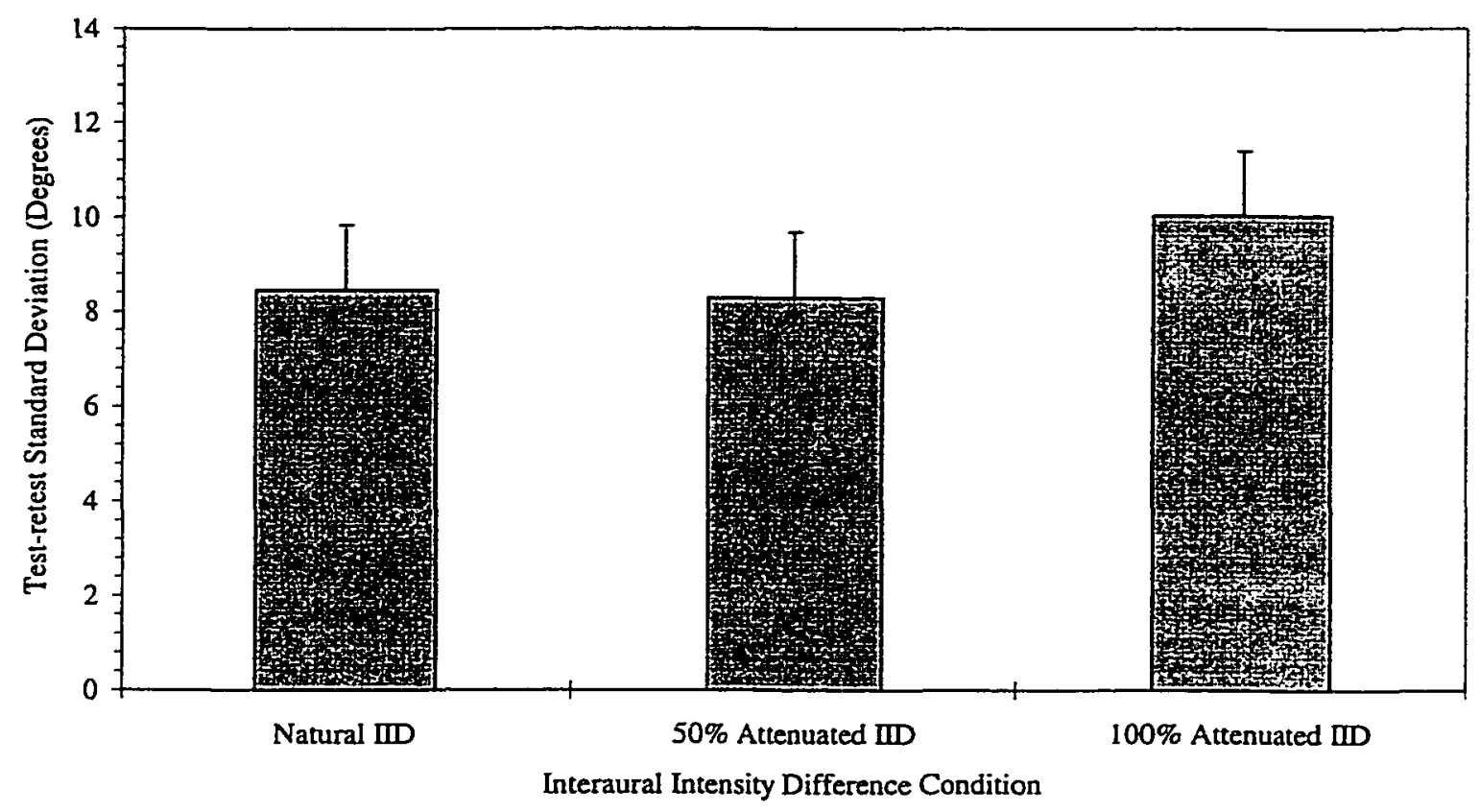

Figure 27: Effect of interaural intensity difference condition on test-retest standard deviation for band-pass noises. Error bars represent a difference at the 0.05 level of significance using Tukey's HSD. 


\subsubsection{Azimuth}

The main factor azimuth was significant for both of the signal types $(p<0.01)$.

These results are shown in Figure 28. The plot makes it evident that $\sigma_{E}$ was greater for azimuths to the sides $\left(>33.75^{\circ}\right.$ or $\left.<-33.75^{\circ}\right)$ than for those in front for both signal types.
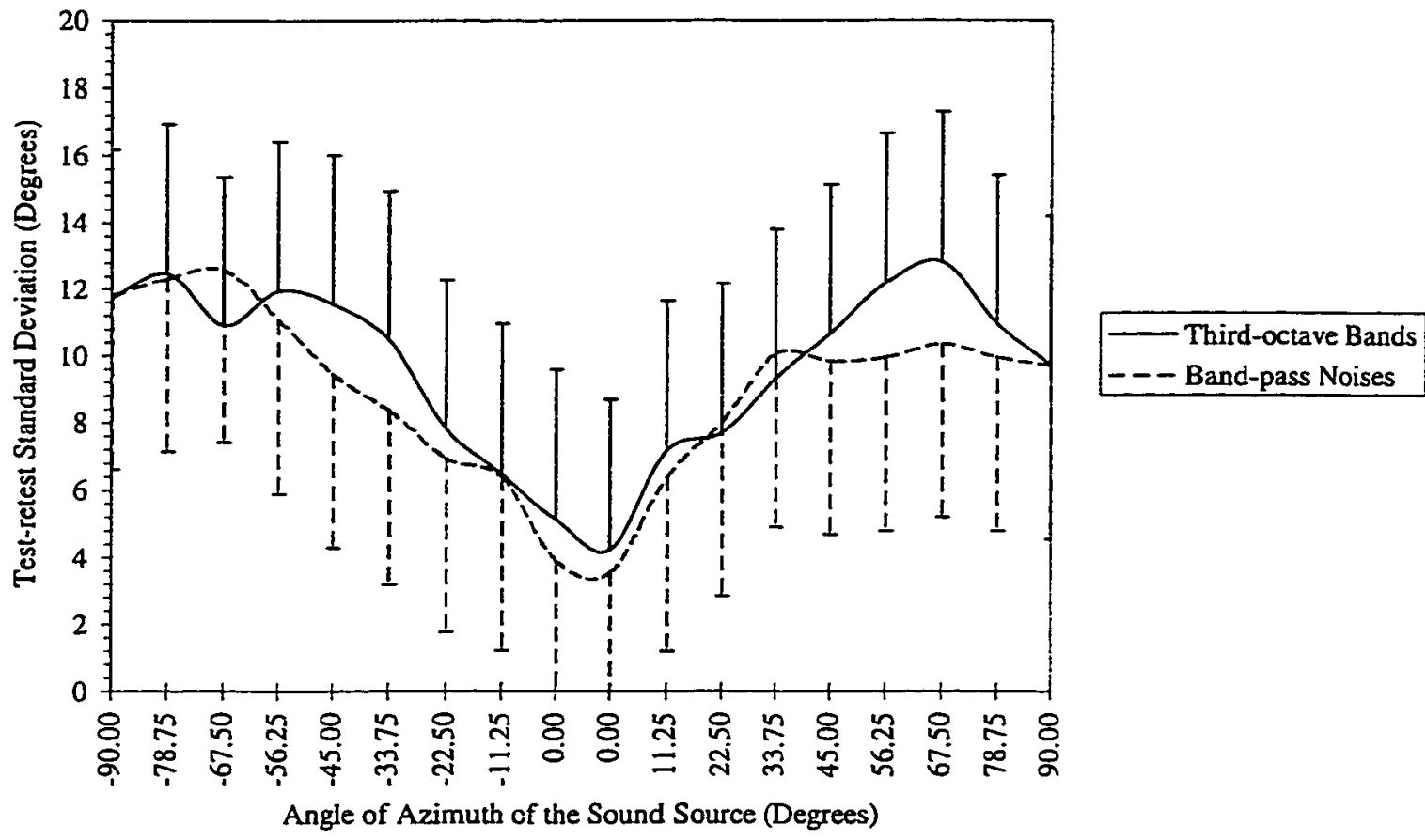

Figure 28: Effect of azimuth on test-retest standard deviation. Error bars represent a difference at the 0.05 level of significance using Tukey's HSD. For clarity of presentation, error bars are shown in the positive direction for third-octave bands and in the negative direction for band-pass noises.

\subsubsection{Azimuth and Signal Frequency}

The interaction of azimuth and signal frequency was significant for both signal types $(p<0.05)$. These interactions are plotted in Figure 29. The interaction for the thirdoctave band noises appears to be based upon the greater values of $\sigma_{E}$ for the left side relative to the right side for $0.5 \mathrm{kHz}$ and $1.0 \mathrm{kHz}$. The interaction for the wide band noises appears to be based upon differences on the right side for the 2.0 to $4.0 \mathrm{kHz}$ signal, 
particularly at azimuths $33.75^{\circ}$ and $78.75^{\circ}$. However, there is a great deal of variability in these plots which makes them difficult to interpret.

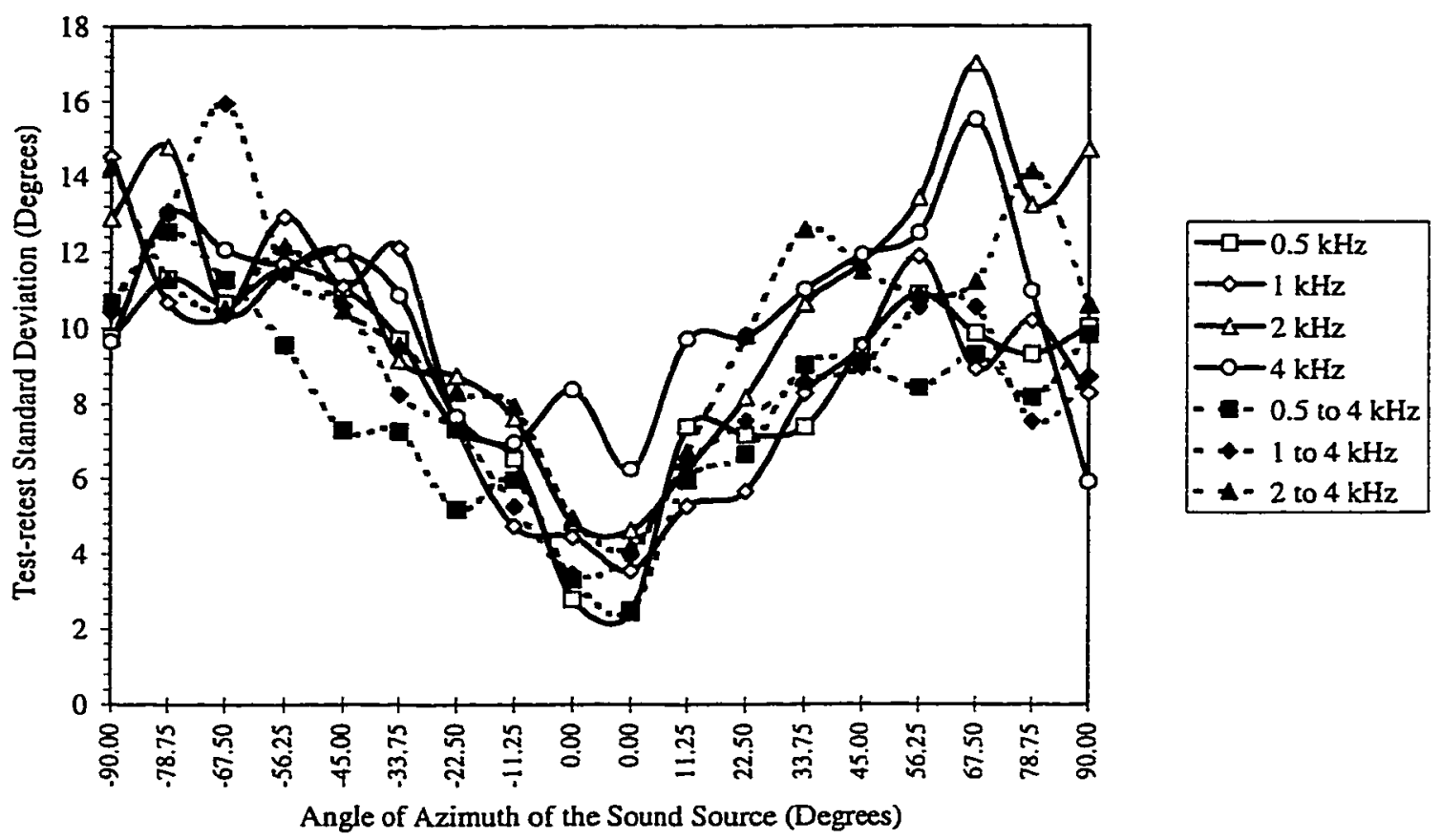

Figure 29: Effect of azimuth and signal frequency on test-retest standard deviation. For clarity of presentation, error bars are omitted.

\subsubsection{Azimuth and Interaural Intensity Difference Condition}

The interaction between azimuth and interaural intensity difference condition is significant for both signal types $(\mathrm{p}<0.01)$. Refer to Figure 30 and Figure 31 for plots of these interactions. For the third-octave bands, it seems clear that the significant interaction is based upon a peak in the value of $\sigma_{E}$ at $67.50^{\circ}$ to the right. Analysis of the interaction over frequency, shown in the Appendix as Figure 85 through Figure 91 (the interaction azimuth by interaural intensity difference condition by signal frequency was also significant for the third-octave bands, $\mathrm{p}<0.01)$ ) indicates that this anomalous peak occurs only for the $2.0 \mathrm{kHz}$ and to a lesser extent for the $4.0 \mathrm{kHz}$ third-octave band. For 
the band-pass noises, the interaction appears attributable mainly to elevated values for $\sigma_{E}$ at the leftmost azimuths $-67.50^{\circ}$ to $-90.00^{\circ}$.

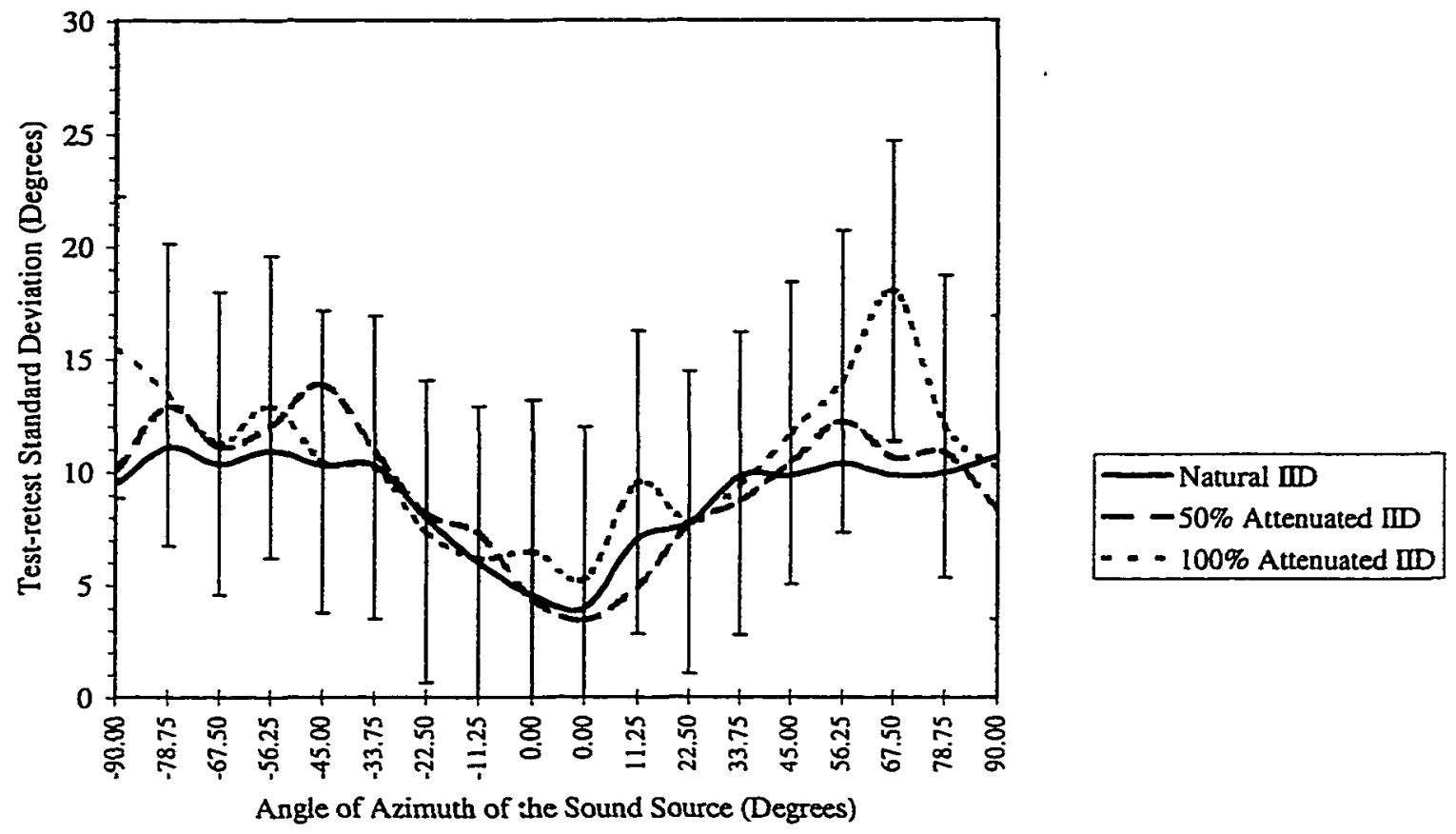

Figure 30: Effect of azimuth and interaural intensity difference condition on testretest standard deviation for the third-octave bands of noise. Error bars represent a difference at the 0.05 level of significance using Tukey's HSD. For clarity of presentation, error bars are displayed only for the $100 \%$ attenuated interaural intensity difference condition. 


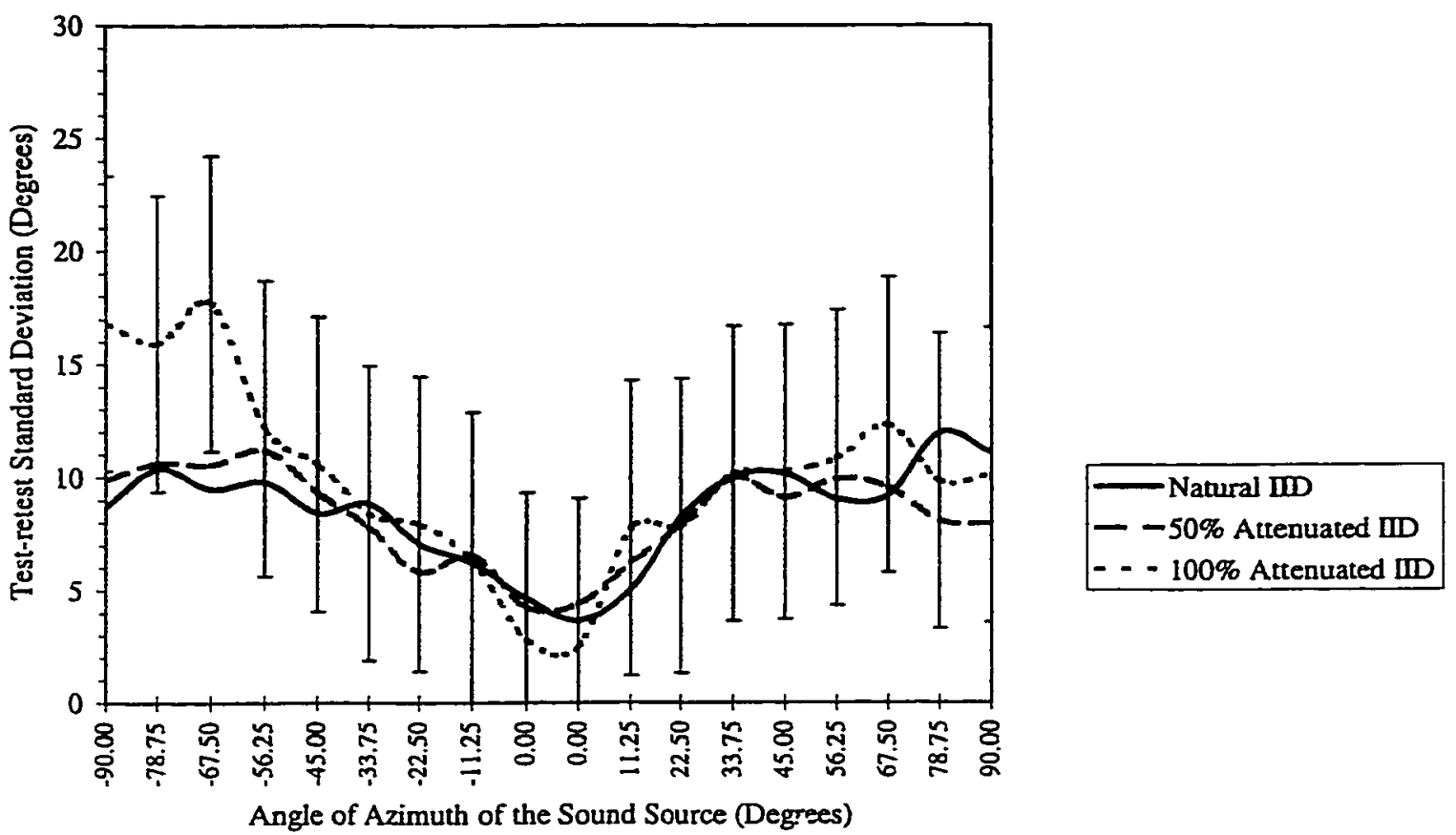

Figure 31: Effect of azimuth and interaural intensity difference condition on testretest standard deviation for the band-pass noises. Error bars represent a difference at the 0.05 level of significance using Tukey's HSD. For clarity of presentation, error bars are displayed only for the $\mathbf{1 0 0} \%$ attenuated interaural intensity difference condition.

\subsection{Summary}

\subsubsection{Room}

The results were the same for sounds recorded in the minitheater as they were for those recorded in the anechoic chamber.

\subsubsection{Interaural Intensity Difference Condition for Signals with Different Frequency Content}

The results of this experiment can be summarized by reference first to the scatterplots of sound source and subject response shown in Figure 10 and Figure 11, as well as in the Appendix in Figure 50 through Figure 70. Localization accuracy was affected negatively by the elimination of interaural intensity differences, but not by reducing them by one half of their original levels in $\mathrm{dB}$. This effect was different for 
signals with different frequency content and bandwidth. Third-octave band signals with low-frequency content ( $1.0 \mathrm{kHz}$ and below) were affected less than signals containing information higher than $1.0 \mathrm{kHz}$. The greatest effect was seen for the localization of 2.0 $\mathrm{kHz}$ third-octave bands of noise. For signals of greater bandwidth, the effect was seen regardless of the presence of low-frequency information.

Analyses of variance for the root mean square error $\left(\sigma_{e}\right)$ confirmed these observations. There was no significant effect of elimination of interaural intensity differences for a $0.5 \mathrm{kHz}$ third-octave band, but for the higher frequency bands the effect was significant. Root mean square errors had the greatest magnitude for the $2.0 \mathrm{kHz}$ signal. The effect of attenuating the interaural intensity difference by $100 \%$ was significant, however, for all of the band-pass noises, including the 0.5 to $4.0 \mathrm{kHz}$ band which contained substantial low-frequency information. There was no significant effect of reducing interaural intensity differences by half of their level in $\mathrm{dB}$ for any of the signals. Analysis of the test-retest standard deviation $\left(\sigma_{E}\right)$ led to similar conclusions. While there was no significant effect of the $100 \%$ attenuated condition on the variability of localization judgments for third-octave bands, the variability of localization judgments for the band-pass noises under the $100 \%$ attenuated condition was significantly greater than that for the unattenuated condition.

\subsubsection{Azimuth and Interaural Intensity Difference Condition}

As expected, the accuracy of all localization judgments decreased with increasing angle of azimuth of the sound source left or right of the center. Furthermore, the decrease in localization accuracy associated with attenuating interaural intensity differences by $100 \%$ was observed to increase with increasing angle of azimuth. An interesting 
observation was that the effect of $100 \%$ interaural intensity difference attenuation was substantially greater on the left side for all of the band-pass noises. All of the subjects exhibited this bias toward greater localization errors on the left side. This effect was also observed in the analysis of bias $\left(\boldsymbol{E}_{b}\right)$, in which the $100 \%$ attenuated condition introduced biases that were positive in sign, indicating a bias to the right. This is of course consistent with a tendency toward greater localization errors for sound sources on the left side. Such errors tended to be in the positive direction; that is, to the right or toward the center. 


\section{CHAPTER 5: DISCUSSION}

\subsection{Comparison of Localization Results with Published Research.}

\subsubsection{Overall Accuracy}

The overall accuracy of horizontal localization for unaltered signals under the conditions of this experiment, i.e., while listening to pre-recorded stimuli from the ears of KEMAR, was similar to that found in published research in which subjects localized sounds presented in a sound field. In this experiment, the average root mean square localization error $\left(\sigma_{e}\right)$ for the unaltered third-octave bands of noise was about $13^{\circ}$. For the widest of the band-pass noises, $\sigma_{e}$ was $9.4^{\circ}$. These results compare favorably with those of Good and Gilkey (1996), Gilkey and Anderson (1995), Butler, et al. (1990) and Wightman and Kistler (1989) for the localization of wide band signals. The error values (root mean square or unsigned error) obtained in these studies are shown in Table 8.

The response methodologies varied among the above-mentioned studies. Butler, et al. (1990) and Wightman and Kistler (1989) used a method in which subjects identified sound sources using an absolute judgment, calling out numerical estimates of horizontal and vertical coordinates. Good and Gilkey (1996) and Gilkey and Anderson (1995) used an approach called God's eye localization pointing (GELP) in which the subject pointed to the apparent sound source location on a $20-\mathrm{cm}$ spherical model of auditory space. In the Butler, et al. study (1990) and the present study, subjects named the number of the loudspeaker from which the sound seemed to originate. 
Table 8: Horizontal localization errors resulting from recently published studies.

\begin{tabular}{|c|c|c|c|c|c|c|c|c|}
\hline Authors & Date & $\begin{array}{c}\text { Speaker } \\
\text { Separation } \\
\text { (Degrees) }\end{array}$ & $\begin{array}{l}\text { Range of } \\
\text { Speakers } \\
\text { (Degrees) }\end{array}$ & $\begin{array}{c}\text { Planes: } \\
\text { Horiz (H) } \\
\text { Vertical } \\
\text { (V) } \\
\end{array}$ & Method & Stimulus & \begin{tabular}{|c|} 
Root Mean \\
Square \\
Error \\
(Degrees) \\
\end{tabular} & $\begin{array}{l}\text { Average } \\
\text { Unsigned } \\
\text { Error } \\
\text { (Degrees) }\end{array}$ \\
\hline Bakke & 1998 & 11.25 & 180 & $\mathrm{H}$ & $\begin{array}{l}\text { Speaker } \\
\text { number }\end{array}$ & \begin{tabular}{|l|} 
Third-octave \\
Bands (340 \\
ms)
\end{tabular} & 13 & 11 \\
\hline Bakke & 1998 & 11.25 & 180 & $\mathrm{H}$ & $\begin{array}{l}\text { Speaker } \\
\text { number }\end{array}$ & $\begin{array}{l}0.5 \text { to } 4.0 \\
\text { kHz WBN } \\
(340 \mathrm{~ms}) \\
\end{array}$ & 9.4 & 7.4 \\
\hline Butler, et al. & 1990 & 15 & 180 & $\mathrm{HV}$ & \begin{tabular}{|l|}
$\begin{array}{l}\text { Speaker } \\
\text { number }\end{array}$ \\
\end{tabular} & $\begin{array}{l}\text { White noise } \\
\text { bursts }\end{array}$ & & 10 \\
\hline $\begin{array}{l}\text { Good \& } \\
\text { Gilkey }\end{array}$ & 1996 & 15 & 360 & $\mathrm{HV}$ & GELP & $\begin{array}{l}\text { Broad band } \\
\text { click train }\end{array}$ & 13 & $\begin{array}{r}\text { Not } \\
\text { Available }\end{array}$ \\
\hline $\begin{array}{l}\text { Gilkey \& } \\
\text { Anderson }\end{array}$ & 1995 & 15 & 360 & $\mathrm{HV}$ & GELP & $\begin{array}{l}\text { Broad band } \\
\text { click train }\end{array}$ & 15 & $\begin{array}{r}\text { Not } \\
\text { Available }\end{array}$ \\
\hline $\begin{array}{l}\text { Gilkey \& } \\
\text { Anderson }\end{array}$ & 1995 & 15 & 360 & $\mathrm{HV}$ & GELP & Speech & 16 & $\begin{array}{r}\text { Not } \\
\text { Available }\end{array}$ \\
\hline $\begin{array}{l}\text { Wightman \& } \\
\text { Kistler }\end{array}$ & 1989 & 15 & 225 & $\mathrm{HV}$ & $\begin{array}{l}\text { Coordinate } \\
\text { system }\end{array}$ & $\begin{array}{l}0.2 \text { to } 14 \mathrm{kHz} \\
\text { WBN }(250 \\
\mathrm{ms})\end{array}$ & $\begin{array}{r}\text { Not } \\
\text { Available }\end{array}$ & 17 \\
\hline
\end{tabular}

Two other studies reported significantly smaller localization errors. Makous and Middlebrooks (1990) and Oldfield and Parker (1984) used experimental methods that included pointing responses. Subjects oriented their heads toward the perceived sound source location in the Makous and Middlebrooks (1990) experiment, and aimed a gun at the sound source in the Oldfield and Parker (1984) study. The resulting unsigned localization errors were $\sim 5^{\circ}$ and $\sim 6^{\circ}$, respectively, using broad-band auditory stimuli. These results are shown in Table $9^{5}$.

\footnotetext{
${ }^{5}$ All of the experiments referenced in Table 8 and Table 9 (except for the present experiment) were performed in a sound field using an array of loudspeakers in both the horizontal and vertical planes using broad band stimuli. The data reported in these tables are calculated from published responses to stimuli presented in the front of the subject (within $\pm 90^{\circ}$ azimuth) at close to ear level.
} 
Table 9: Horizontal localization errors obtained using a pointing response method.

\begin{tabular}{|l|r|r|r|r|r|r|r|}
\hline \multicolumn{1}{|c|}{ Authors } & Date & $\begin{array}{c}\text { Speaker } \\
\text { Separation } \\
\text { (Degrees) }\end{array}$ & $\begin{array}{c}\text { Range of } \\
\text { Speakers } \\
\text { (Degrees) }\end{array}$ & $\begin{array}{c}\text { Planes: } \\
\text { Horiz (H) } \\
\text { Vertical } \\
\text { (V) }\end{array}$ & Method & Stimulus & $\begin{array}{c}\text { Average } \\
\text { Unsigned } \\
\text { Error } \\
\text { (Degrees) }\end{array}$ \\
\hline $\begin{array}{l}\text { Makous \& } \\
\text { Middlebrooks }\end{array}$ & 1990 & 10 & 360 & $\mathrm{HV}$ & Head Orient & $\begin{array}{l}1.8 \text { to } 16 \\
\mathrm{kHz} \text { WBN } \\
\text { (150 ms) }\end{array}$ & \\
\hline Oidfield \& Parker & 1984 & 10 & 360 & $\mathrm{HV}$ & Pointing & White noise & \\
\hline
\end{tabular}

Table 8 and Table 9 suggest that the use of head orientation or pointing response methods resulted in greater localization accuracy than the use of more abstract paradigms such as pointing to a model of auditory space, calling out coordinates, or identifying the number of the sound source loudspeaker. A possible explanation for the lower values of localization error with a pointing paradigm is the fact that the subjects' responses are not restricted. For example, if the responses are constrained to identifying one of a set of loudspeakers, then a quantization error is introduced. The variance of the quantization error is equal to $\frac{1}{12}$ (speaker separation) ${ }^{2}$. Under these conditions of constraint, the error variance $\left(\sigma_{E}^{2}\right)$ is equal to the sum of the test-retest variance and the quantization error variance. If, on the other hand, the subject is not constrained to a finite set of loudspeakers, no quantization error is introduced and the measured localization error will be smaller.

It should be noted that the subjects in the two experiments that used pointing techniques were not able to turn their heads toward the sound sources during the test procedures, and were therefore restrained from using head movement cues to assist in localizing the target sounds. In the Oldfield and Parker (1984) study, the subjects' heads were held fixed by a chin brace while they pointed to the sound source with a gun. In the 
Makous and Middlebrooks (1990) study, the sound bursts were so brief as to preclude the use of dynamic head-movement cues.

Another consideration is the fact that higher level cognitive re-mapping of the perceived sound location into an abstract verbal (or motor, in the case of GELP) response is not necessary for a hand- or head-pointing response.

The wider bandwidth of the signals used in Makous and Middlebrooks (1990) and Oldfield and Parker (1984) relative to the limited bandwidth of the signals used in the present study may also be a factor to consider. The former experiments used bandwidths that contained a great deal of high-frequency information and therefore contained a richer set of interaural time difference, interaural intensity difference and spectral cues than the signals used in the present study, which were limited to 0.5 to $4.0 \mathrm{kHz}$.

These differences in methodology (use of wider bandwidth signals and the use of a pointing technique that eliminates quantization error) could account for the smaller error values obtained by Makous and Middlebrooks (1990) and Oldfield and Parker (1984) as compared to the present study and other recent research.

\subsubsection{Effect of Azimuth on Localization Accuracy}

The accuracy of localization (as specified by the root mean square error, $\left(\sigma_{e}\right)$ for the unaltered wide band $(0.5 \mathrm{kHz}$ to $4.0 \mathrm{kHz})$ signals in the present experiment varied substantially with azimuth. This is shown in Figure 32. Localization errors increased with greater distance from the center. At $90^{\circ}, \sigma_{e}$ increased as a result of an artifactual end effect. (This is discussed in further detail below.) These results are consistent with the minimum audible angle (MAA) data of Mills (1958), who found that the resolving power 
of localization was greatest for $0^{\circ}$ azimuth and became increasingly poorer as angle of azimuth increased.

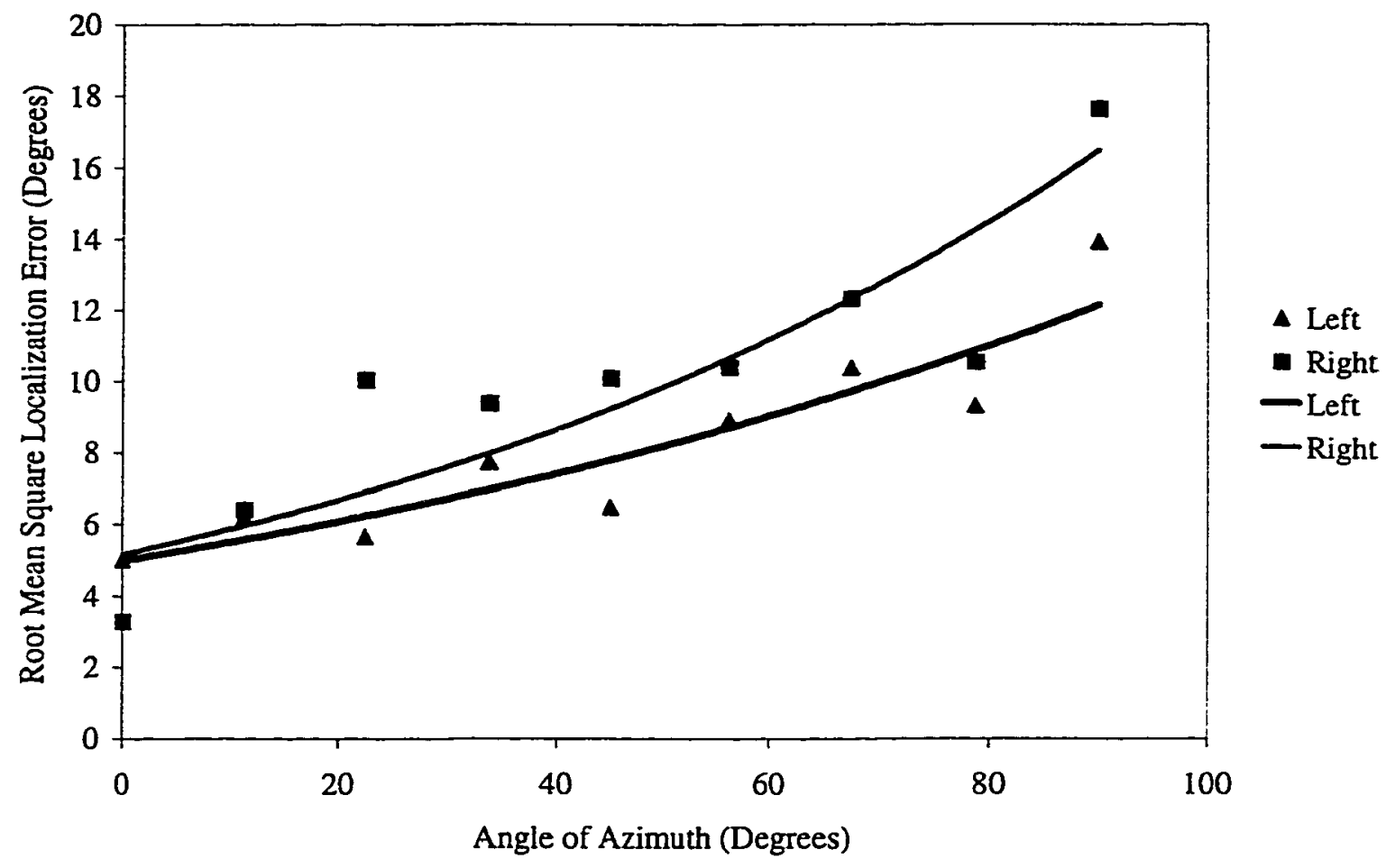

Figure 32: Relationship between azimuth and root mean square localization error $\left(\sigma_{e}\right)$ for the present experiment. Results for the left and right side are shown separately. The signal is a band-pass noise, $0.5 \mathrm{kHz}$ to $4.0 \mathrm{kHz}$ in width. Trend lines are exponentially fit to the data on the left and right sides.

Butler et al. (1990) reported a similar relationship between localization accuracy and azimuth. Horizontal localization error for the binaural listening condition increased with increasing distance from the center. Interestingly, there were greater errors for the $0^{\circ}$ position relative to adjacent positions. This is probably a result of the fact that testing was performed only on the left side. The $0^{\circ}$ position in the loudspeaker array was at the extreme end of the array, resulting in an end effect at $0^{\circ}$ azimuth. Data from Makous and Middlebrooks (1990) showed a similar pattern of increasing error with distance from the center, although their absolute horizontal localization errors were generally smaller. In 
contrast, the results of Oldfield and Parker (1984) showed no such pattern up to azimuths of $80^{\circ}$. Data from these authors are displayed in Figure 33. The results of Wightman and Kistler (1989) indicated no greater localization accuracy within a central region $\left( \pm 45^{\circ}\right)$ relative to the sides $\left(>45^{\circ}\right)$, and are thus not in agreement with the results of the present study. The Wightman and Kistler (1989) data are not shown on Figure 33 because they were reported only as average values over broad ranges of azimuth and elevation.

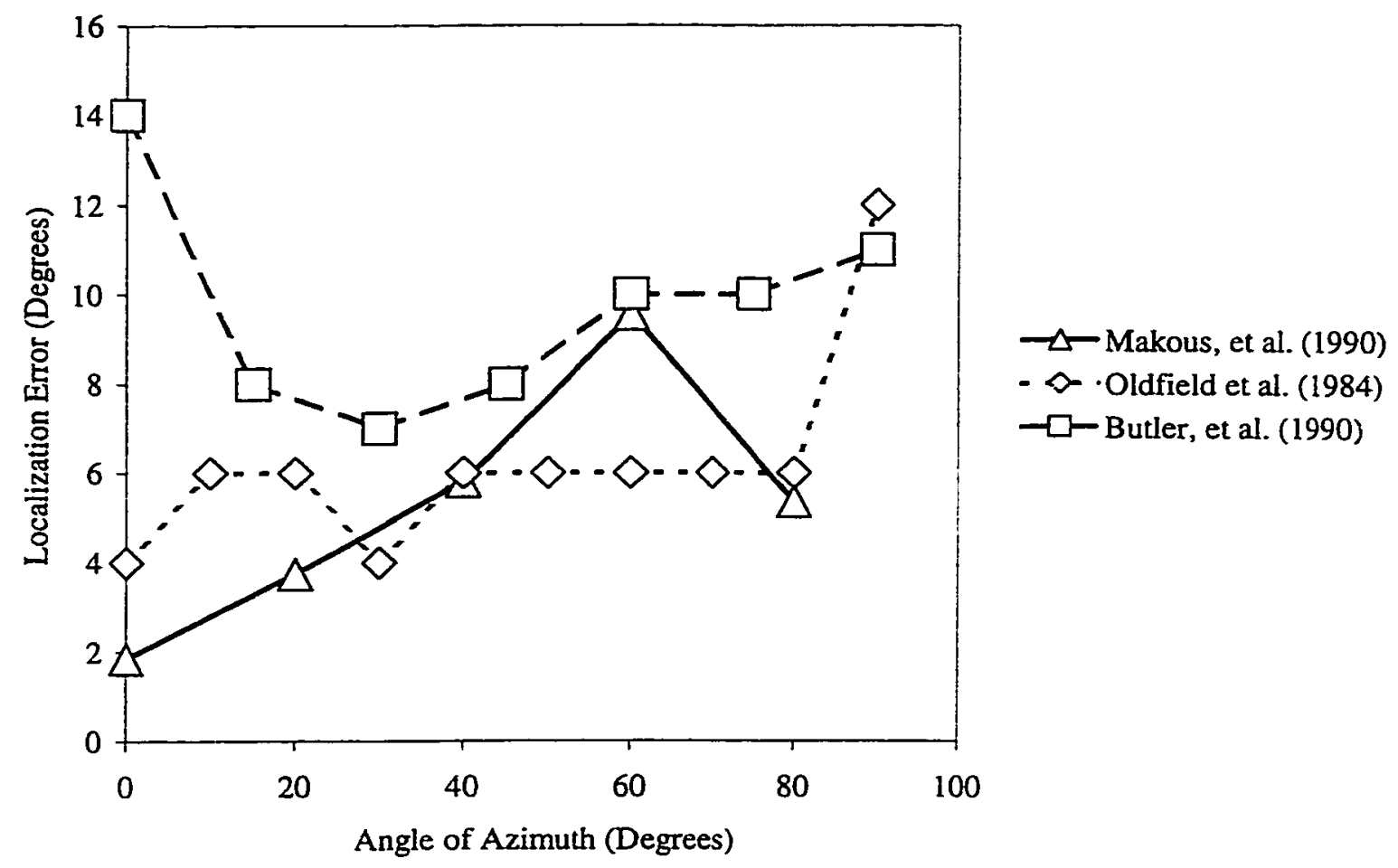

Figure 33: Relationship between azimuth and localization errors for three published studies that used broad band stimuli.

Note the increase in the magnitude of the localization error $\left(\sigma_{e}\right)$ for the $90^{\circ}$ azimuth loudspeaker location on both the right and left sides in the present data, plotted in Figure 32. It is important to keep in mind the fact that the $90^{\circ}$ loudspeaker positions represented the extreme ends of the array of possible sound source locations and thus introduced an end effect. This effect produced an bias in that apparent locations greater 
than $90^{\circ}$ yielded a response of $90^{\circ}$, thereby increasing the number of $90^{\circ}$ responses. The magnitude of the effect is shown in Figure 34 and Figure 35. These figures are stacked area graphs that illustrate how observer precision $\left(\sigma_{E}\right)$, bias $\left(E_{b}\right)$ and accuracy $\left(\sigma_{e}\right)$ change with speaker location. The absolute values of the bias on the left and right side were averaged to provide the data for these figures.

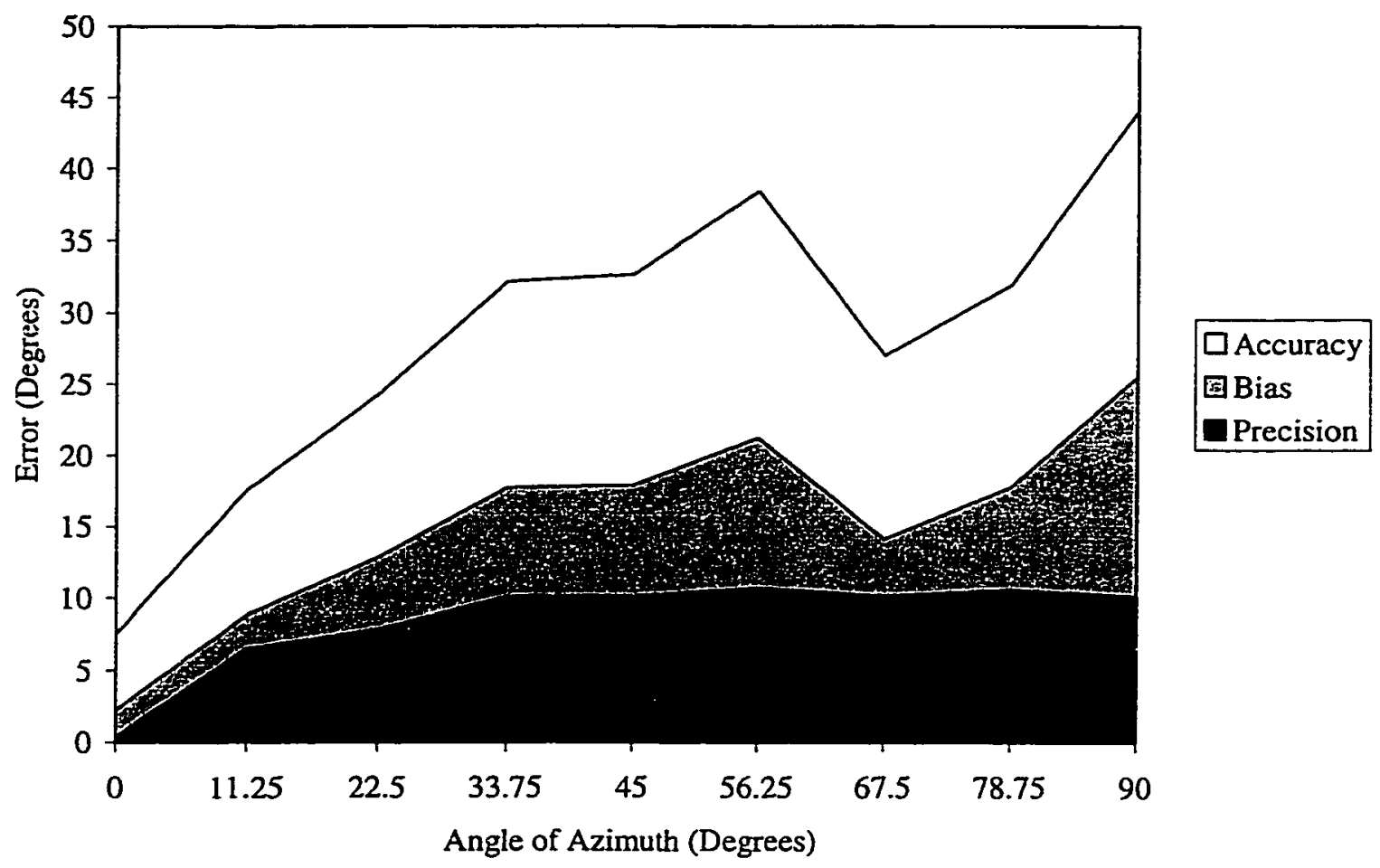

Figure 34: The contribution of precision and bias to localization accuracy for thirdoctave bands of noise. These values are averaged over the four third-octave bands. The condition is natural interaural level differences. 


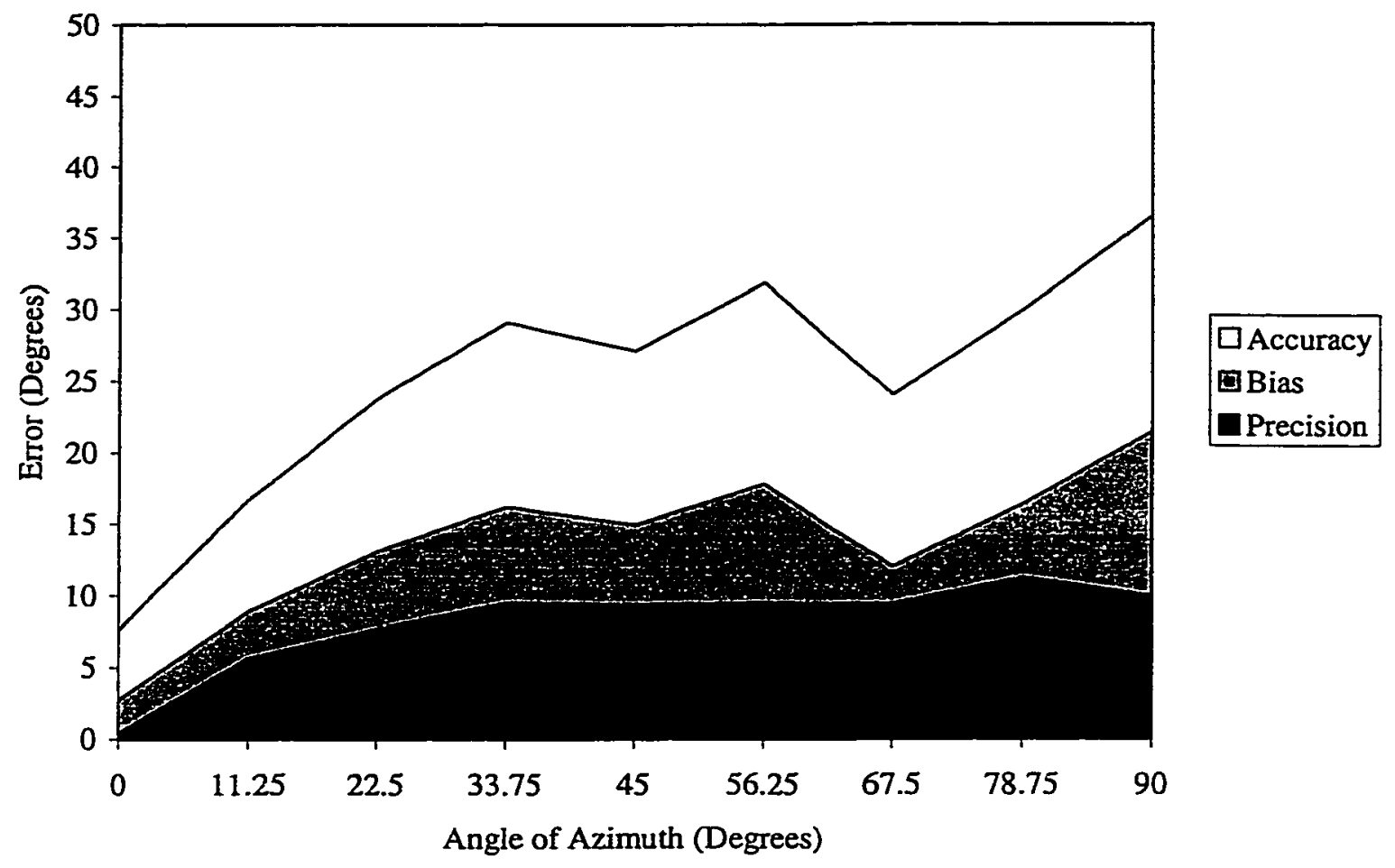

Figure 35: The contribution of precision and bias to localization accuracy for bandpass noises. These values are averaged over the three band-pass noises. The condition is natural interaural level differences.

Consideration of the nature of this end effect highlights the importance of an analysis that looks at the precision and the bias of localization judgments in addition to the overall accuracy. Note that the precision of localization is very small at the center, increases gradually out to about $33^{\circ}$ and then remains relatively constant for angles of azimuth greater than $\sim 33^{\circ}$. On the other hand, there is a distinct rise in bias for the $90^{\circ}$ position. This is true for both the third-octave band noises and the band-pass noises.

\subsubsection{Effect of Signal Frequency on Localization Accuracy}

Localization accuracy in the present experiment was also affected by the center frequency of the third-octave bands. Bands of noise at $0.5 \mathrm{kHz}, 1.0 \mathrm{kHz}$ and $4.0 \mathrm{kHz}$ were localized with significantly greater accuracy than those at $2.0 \mathrm{kHz}$. Mills (1958) found that there was a dramatic increase in minimum audible angle for frequencies 
between $1.0 \mathrm{kHz}$ and $3.0 \mathrm{kHz}$ with a maximum peak just below $2.0 \mathrm{kHz}$ and recovery above $3.0 \mathrm{kHz}$. The pattern of localization errors seen in the present experiment for signals with natural interaural intensity differences is consistent with the findings of Mills (1958) in that spatial resolution is poorer in the $2.0 \mathrm{kHz}$ frequency region.

Giguère and Abel (1993) studied the horizontal localization of third-octave bands of noise with the same center frequencies as those of the present experiment in both an absorbent and a reverberant room. They reported their data as percent correct responses using two loudspeaker arrays (frontal and lateral) in which speakers were separated by $30^{\circ}$. Only the data from their frontal array are discussed here. There were two replications of 20 trials at each speaker location in the two different rooms, for a total of 40 trials at each speaker location. In order to compare the present results with those of Giguère and Abel (1993), it was necessary to calculate percent correct responses within a $33.75^{\circ}$ arc for azimuths centered around the speaker locations closest to $15^{\circ}, 45^{\circ}$ and $75^{\circ}$ on the left and right.

Figure 36 shows the data of Giguère and Abel (1993) and the data from the present experiment. For purposes of this comparison, the responses from this experiment were considered "correct" if they were within two speaker locations $\left( \pm 22.50^{\circ}\right)$ of the target. This manipulation was necessary because of the large speaker separation used in the Giguère and Abel (1993) study $\left(30^{\circ}\right)$. 


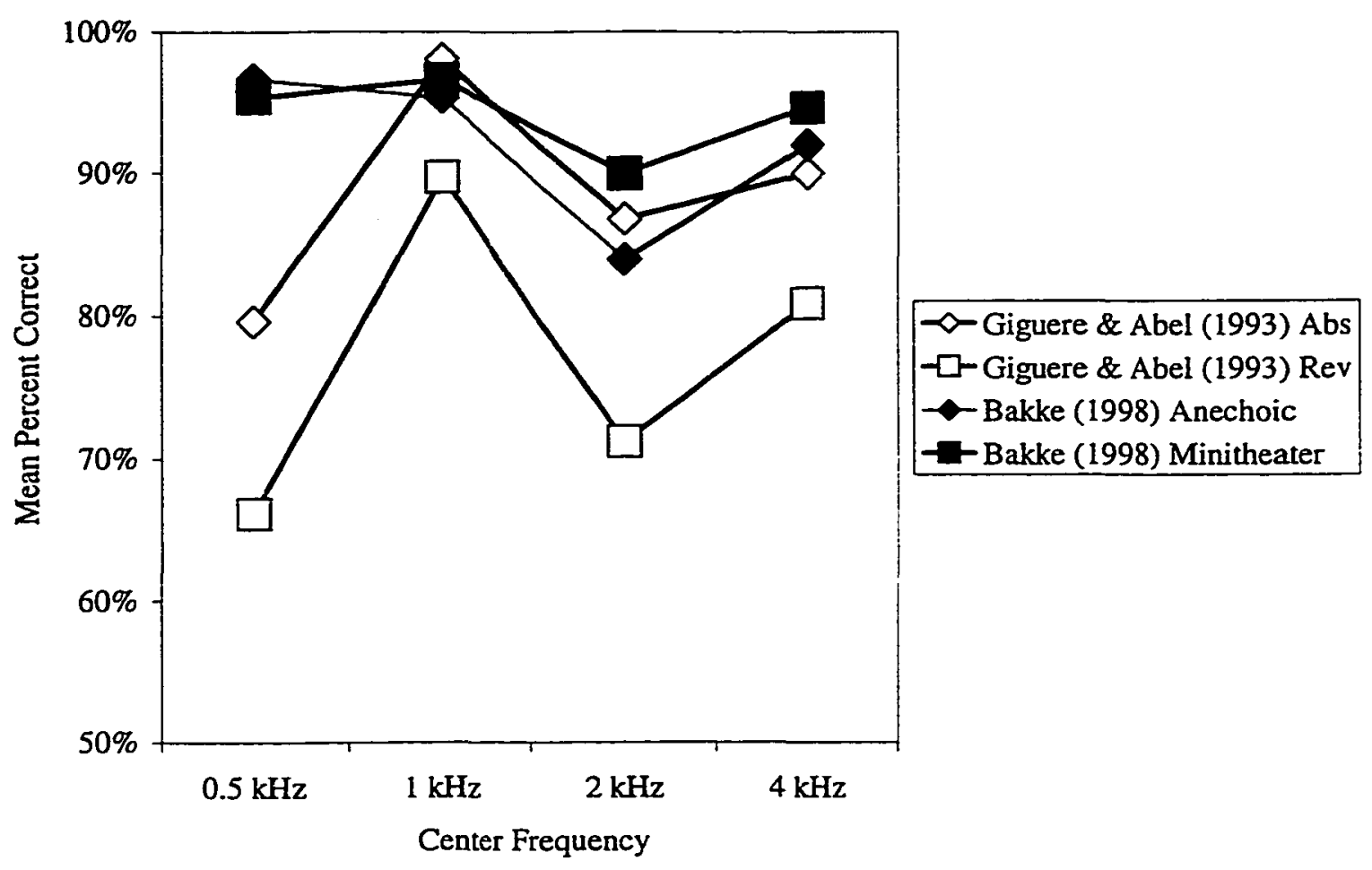

Figure 36: The overall percent correct localization scores of Giguère and Abel (1993) for an absorbent and a reverberant room with the results of the present experiment for the anechoic room and minitheater.

While Giguère and Abel (1993) observed a difference between their absorbent and reverberant rooms, there was no significant effect of Room in the present study. The different results may be explained by the differences in reverberation time and room size between the reverberant room used in the former study and the minitheater used in the present experiment. The reverberant room used by Giguère and Abel (1993) had a reverberation time ranging from $600 \mathrm{msec}$ (at 2.0 and $4.0 \mathrm{kHz}$ ) to $1000 \mathrm{msec}$ (at $0.5 \mathrm{kHz}$ ) and a volume of $21.65 \mathrm{~m}^{2}$. Recall that the reverberation time of the minitheater in the present study was 400 milliseconds (RT-60) and the volume of the room was $337.57 \mathrm{~m}^{2}$. The distance of the loudspeaker from the recording microphones was 1 meter for both experiments. The critical distance for the reverberant room in the current experiment was approximately $5.8 \mathrm{~m}$, so that the 1 meter distance was well within the critical distance (at 
which the reverberant field and direct sound field are equal) and the direct sound dominated the signal. The critical distance for the room in the Giguère and Abel (1993) experiment was approximately 1 meter, so the reverberant sound field is likely to have dominated the signals heard by the subjects. If the loudspeaker in the reverberant room of the present study had been farther than about 6 meters from KEMAR, the reverberant field would have had a greater impact upon the subjects' localization performance.

Another difference between the results of the two studies is the decrement in performance for the $500 \mathrm{~Hz}$ third-octave band noise that was observed for both rooms in the former study but not observed in the present experiment. It appears that most of the decrement in the Giguère and Abel (1993) experiment occurred at the $\pm 75^{\circ}$ speaker positions, which were the end positions of the frontal array in that study. Although the reason for the difference in results between Giguère and Abel (1993) and the present experiment is not clear, it is possible that there was an end effect for the $500 \mathrm{~Hz}$ bandpass noise in the former experiment.

\subsection{Experimental Hypotheses}

\subsubsection{Relative Dominance of Interaural Time Difference and Interaural Intensity Difference Cues}

In a discussion of the results of two of their conflicting cue experiments, Wightman and Kistler $(1993,1997)$ proposed the theory that a reliable localization cue will dominate the determination of apparent source position over a less reliable cue. According to this theory, interaural time difference cues are more reliable than interaural intensity difference cues. For situations in which both of these cues are salient, the interaural time difference cues will dominate auditory localization judgments. This theory was discussed in more detail above, in the Review of the Literature, Section 2.11. 
While this theory explained the results of Wightman and Kistler $(1993,1997)$, Hartmann and Fontana (1991) found that when interaural intensity differences in $0.5 \mathrm{kHz}$ tones were amplified by 10 to $15 \mathrm{~dB}$ over their naturally occurring values, a significant localization bias was introduced.

Table 10: Comparisons Among the Results of Several Interaural Intensity Difference Manipulation Studies

\begin{tabular}{|l|l|l|l|}
\hline \multicolumn{1}{|c|}{ Experiment } & \multicolumn{1}{|c|}{$\begin{array}{c}\text { Interaural Intensity } \\
\text { Difference } \\
\text { Manipulation }\end{array}$} & \multicolumn{1}{|c|}{ Eignal } & \multicolumn{1}{|c|}{} \\
\hline $\begin{array}{l}\text { Wightman \& Kistier, } \\
1997\end{array}$ & $100 \%$ Attenuation & $\begin{array}{l}\text { Wide-band noise 0.2 to } \\
14 \mathrm{kHz}\end{array}$ & None \\
\hline Bakke, 1998 & $50 \%$ Attenuation & $\begin{array}{l}0.5 \mathrm{kHz} \text { Third-octave } \\
\text { Band Noise }\end{array}$ & None \\
\hline Bakke, 1998 & $100 \%$ Attenuation & $\begin{array}{l}0.5 \mathrm{kHz} \text { Third-octave } \\
\text { Band Noise }\end{array}$ & None \\
\hline Bakke, 1998 & $50 \%$ Attenuation & $\begin{array}{l}1.0,2.0,4.0 \mathrm{kHz} \text { Third- } \\
\text { octave Band Noise }\end{array}$ & None \\
\hline Bakke, 1998 & $100 \%$ Attenuation & $\begin{array}{l}1.0,2.0,4.0 \mathrm{kHz} \text { Third- } \\
\text { octave Band Noise }\end{array}$ & Bias toward center \\
\hline Bakke, 1998 & $50 \%$ Attenuation & $\begin{array}{l}0.5 \text { to } 4.0 \mathrm{kHz} \text { Band- } \\
\text { pass Noise }\end{array}$ & None \\
\hline Bakke, 1998 & $100 \%$ Attenuation & $\begin{array}{l}0.5 \text { to } 4.0 \mathrm{kHz} \text { Band- } \\
\text { pass Noise }\end{array}$ & Bias toward center \\
\hline $\begin{array}{l}\text { Hartmann and Fontana } \\
\text { (1991) }\end{array}$ & Increased by 5 to 25 dB & $0.5 \mathrm{kHz}$ tones & $\begin{array}{l}\text { Bias toward ear with } \\
\text { greater intensity when } \\
\text { increase exceeded } 10 \text { to } \\
15 \text { dB }\end{array}$ \\
\hline
\end{tabular}

Table 10 summarizes some of the results of the present experiment and compares them with those of Wightman and Kistler (1997) and Hartmann and Fontana (1991). The results of the present experiment for the $0.5,1.0,2.0$ and $4.0 \mathrm{kHz}$ third-octave bands of noise support the theory of Wightman and Kistler (1997). Neither $50 \%$ attenuation or $100 \%$ attenuation of the interaural intensity differences in the $0.5 \mathrm{kHz}$ third-octave band affected auditory localization, i.e., the interaural time difference cues dominated those localization judgments. Nor did attenuation of the interaural intensity differences by $50 \%$ for the $1.0,2.0$ and $4.0 \mathrm{kHz}$ third-octave bands of noise result in localization bias. However, attenuation of interaural intensity differences by $100 \%$ for the latter signals did 
result in localization bias toward the center. Despite the presence of localization bias, this result is consistent with the theory of Wightman and Kistler (1997) because the lowfrequency energy that carries the most salient temporal information is absent from those signals. Giving further support to this theory is the fact that attenuating interaural intensity differences by $50 \%$ did not degrade localization performance for those signals that were limited to the higher frequencies. This result is an indication of the power of interaural time difference cues to influence localization judgments. On the other hand, both the 0.5 to $4.0 \mathrm{kHz}$ band-pass noise used in the present experiment and the $0.5 \mathrm{kHz}$ tones used by Hartmann and Fontana (1991) contained sufficient low-frequency energy to carry salient interaural time difference cues. If interaural time difference cues were dominant in these cases, when such cues were both present and salient, one would have expected these signals to be unaffected by manipulations of interaural intensity difference cues. Thus, it appears that an explanation based on the theory that the most reliable cue will dominate localization judgments is incomplete.

The statement of theory proposed for the current experiment (see Review of the Literature, Section 2.11.1) suggests that the dominance of the most reliable localization cue is not absolute. For horizontal localization, the perception of sound source location will be affected by interaural intensity difference cues even when they conflict with the more reliable interaural time difference cues.

Consideration of the magnitude of the interaural intensity difference for the binaural signal in question may help in understanding how interaural time and intensity difference cues interact in the perception of sound source location. While interaural time difference cues are powerful and will often dominate the perception of sound source 
location when they conflict with interaural intensity difference cues, if the interaural intensity difference exceeds a certain magnitude it may outweigh the interaural time difference, thus dominating the localization judgment.

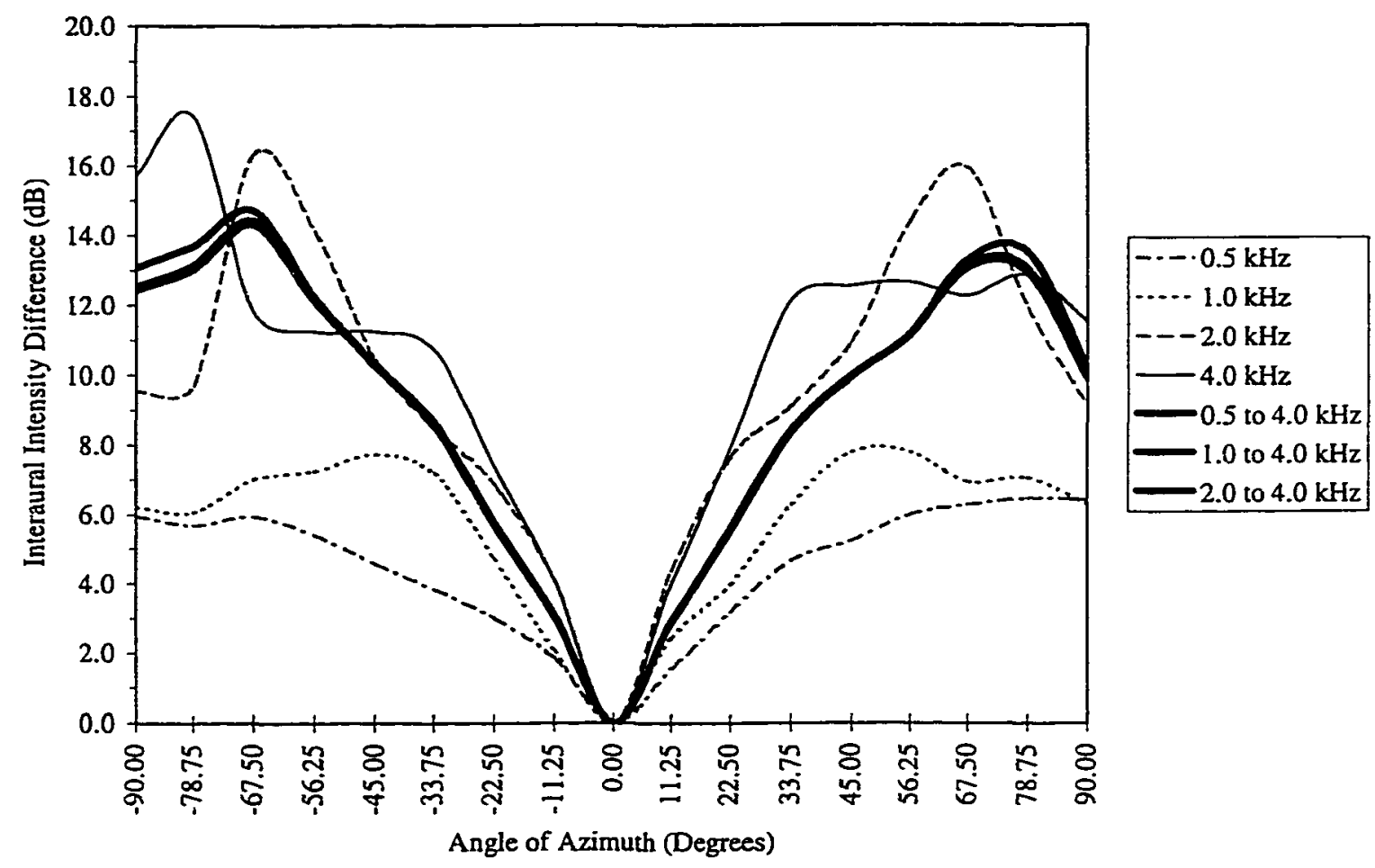

Figure 37: Interaural intensity differences for KEMAR in an anechoic room for the signals used in the present experiment. Data for band-pass signals are shown using the bold lines. 


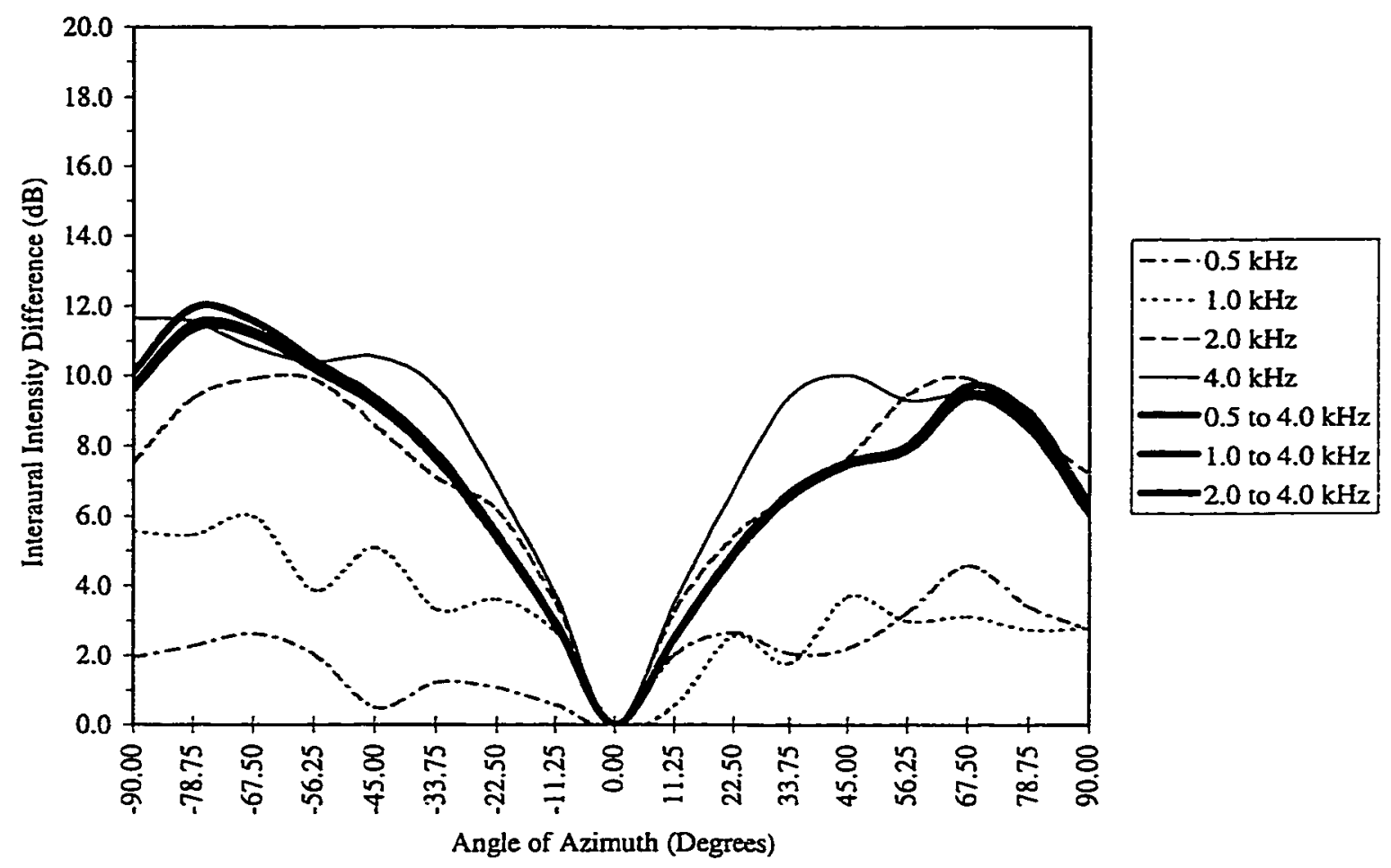

Figure 38: Interaural intensity differences for KEMAR in the minitheater for the signals used in the present experiment. Data for band-pass signals are shown using the bold lines.

The interaural intensity differences for KEMAR under the conditions of the present experiment are shown in Figure 37 for the anechoic room and Figure 38 for the minitheater. These values were derived from the Fast Fourier Transformation data generated by SYSid (@ Copyright 1992 Ariel Corporation, Highland Park, NJ) for each angle of azimuth from $-90^{\circ}$ to $90^{\circ}$ in $11.25^{\circ}$ steps at the time that the experimental signals were recorded. (see Methods, Section 3.1.3) The maximum magnitudes of the interaural intensity differences measured under the conditions of this experiment (at the angle of azimuth at which the interaural intensity difference was greatest) are shown in Table 11 . 
Table 11: Maximum magnitudes (in dB) of interaural intensity differences present in the signals used in this experiment. These values were calculated using the directional response measurements of KEMAR's ears that were made at the time the signals were recorded.

\begin{tabular}{|l|l|l|l|l|l|r|r|}
\hline Room & $\begin{array}{l}0.5 \\
\text { Third- } \\
\text { octave } \\
\text { Band }\end{array}$ & $\begin{array}{l}1.0 \mathrm{kHz} \\
\text { Third- } \\
\text { octave } \\
\text { Band }\end{array}$ & $\begin{array}{l}2.0 \mathrm{kHz} \\
\text { Third- } \\
\text { octave } \\
\text { Band }\end{array}$ & $\begin{array}{l}4.0 \mathrm{kHz} \\
\text { Third- } \\
\text { octave } \\
\text { Band }\end{array}$ & $\begin{array}{l}0.5 \text { to 4.0 } \\
\mathrm{kHz} \text { Band- } \\
\text { Pass Noise }\end{array}$ & $\begin{array}{l}1.0 \text { to 4.0 } \\
\mathrm{kHz} \text { Band- } \\
\text { Pass Noise }\end{array}$ & $\begin{array}{l}\text { l.0 to 4.0 } \\
\mathrm{kHz} \text { Band- } \\
\text { Pass Noise }\end{array}$ \\
\hline Anechoic & 6.5 & 7.8 & 16.3 & 17.4 & 14.3 & 14.4 & 14.7 \\
\hline Minitheater & 4.6 & 6.0 & 9.7 & 11.7 & 11.4 & 11.5 & 12.0 \\
\hline
\end{tabular}

The unattenuated $0.5 \mathrm{kHz}$ third-octave band had interaural intensity differences that did not exceed $6.5 \mathrm{~dB}$ for the anechoic room and $4.6 \mathrm{~dB}$ for the minitheater. The other unattenuated signals had interaural intensity differences of from 7.8 to $17.4 \mathrm{~dB}$ for the anechoic room and 6.0 to $11.7 \mathrm{~dB}$ for the minitheater. Some of these interaural intensity difference values are within the range of those that induced a bias in the data of Hartmann and Fontana (1991). In attenuating the interaural intensity differences to onehalf of their original value the maximum adjustment was $8.7 \mathrm{~dB}$ (one-half of $17.4 \mathrm{~dB}$ at $4.0 \mathrm{kHz}$ ). This modification did not produce a degradation of localization performance. Reducing interaural intensity differences by $100 \%$ required adjustments of from $4.6(0.5$ $\mathrm{kHz}$ third-octave band in the minitheater) to $17.4 \mathrm{~dB}(4.0 \mathrm{kHz}$ third-octave band in the anechoic room). With the exception of the $0.5 \mathrm{kHz}$ signal, these adjustments resulted in significant effects upon localization performance.

Figure 39 shows the average interaural intensity differences present in each unattenuated signal used in the present experiment. Shown are the average interaural intensity difference values of those azimuths for which there was a significant effect of attenuating the interaural intensity differences by $100 \%$, as well as those for which there was no significant effect. This figure indicates that there is a relationship between the likelihood that an effect of interaural intensity difference attenuation (by 100\%) will be 
observed at a given angle of azimuth, and the magnitude of the interaural intensity difference.

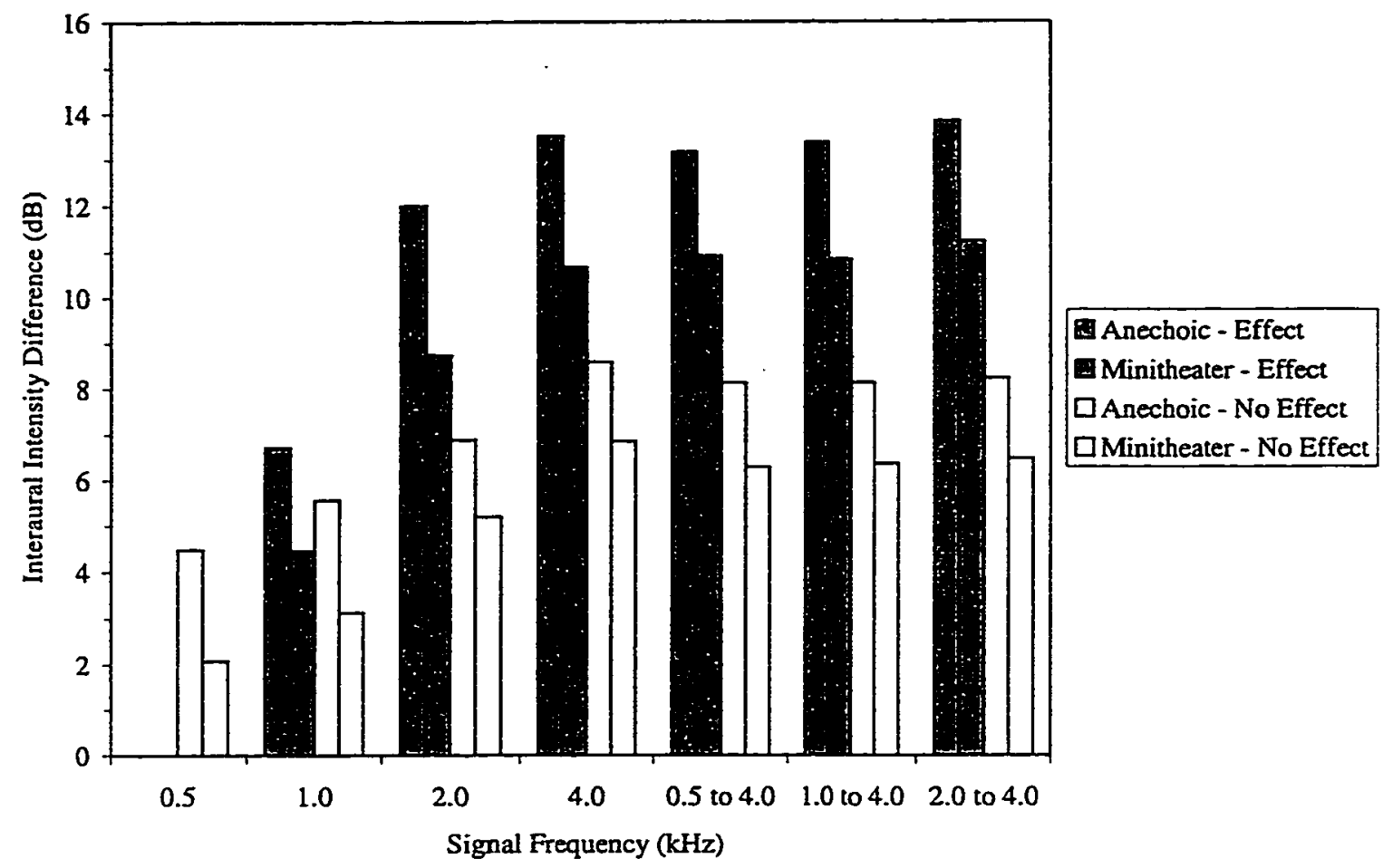

Figure 39: The average interaural intensity differences present in each unattenuated signal used in the present experiment. The darkened columns represent data for azimuths at which there was a significant effect of attenuating the interaural intensity difference by $\mathbf{1 0 0 \%}$, while the light columns represent data for azimuths at which there was no effect of attenuating the interaural intensity differences.

This hypothesis does not explain the results of Wightman and Kistler (1997), however, since the interaural intensity differences in that situation were likely to be greater than $10 \mathrm{~dB}$. It is possible that the added monaural and binaural spectral cues, particularly in the higher frequencies, that exist in the broad band signals used in that experiment provided extra cues that were sufficient for the listeners to override the interaural intensity difference cues that were pointing toward the center. 


\subsubsection{Experimental Hypothesis \#1}

Experimental Hypothesis \#1 states, "Subjects who attempt to identify the horizontal location of the source of sounds in which interaural intensity differences have been attenuated by $50 \%$ or $100 \%$ of their original $\mathrm{dB}$ level will make errors that reflect a bias toward the center."

The major finding of the present experiment is that listeners' horizontal auditory localization accuracy was degraded when interaural intensity difference cues were completely attenuated, but that the attenuation of interaural intensity differences to $50 \%$ of their original value in decibels did not degrade auditory localization accuracy. The magnitude of the root mean square localization error for signals with zero interaural intensity difference was almost double that of signals with natural or attenuated interaural intensity differences. This was true for third-octave band noises as well as for band-pass noises.

An interesting question raised by the results of this experiment is to what extent the degradation in overall localization accuracy reflected an increase in bias versus an overall reduction in the precision of localization. Interaural intensity difference condition was a significant main effect in the analysis of variance performed on the measure of bias but was not a significant effect in the analysis of the measure of precision. This suggests that bias accounted for more of the reduction in accuracy observed in this experiment under the $100 \%$ attenuated condition than did precision of localization. However, our measure of bias (mean localization error) is a signed value, and the fact that the bias has a positive value indicates that the bias was toward the right overall. Thus, consideration of the pooled data for all azimuths leads to the conclusion that bias toward the right side predominated. 
In order to test the hypothesis that attenuating interaural intensity differences results in a bias toward the center, it is necessary to consider the interaction of interaural intensity difference condition with sound source azimuth. Central bias on the left side (i.e., toward the right) could be factored out by equal central bias on the right side (i.e., toward the left), thus leading to an underestimate of the bias. Figure 40 shows the relative contributions of bias and precision to overall accuracy for the right side and the left side. The sign of the bias has been inverted for the left side in order to display positive values.

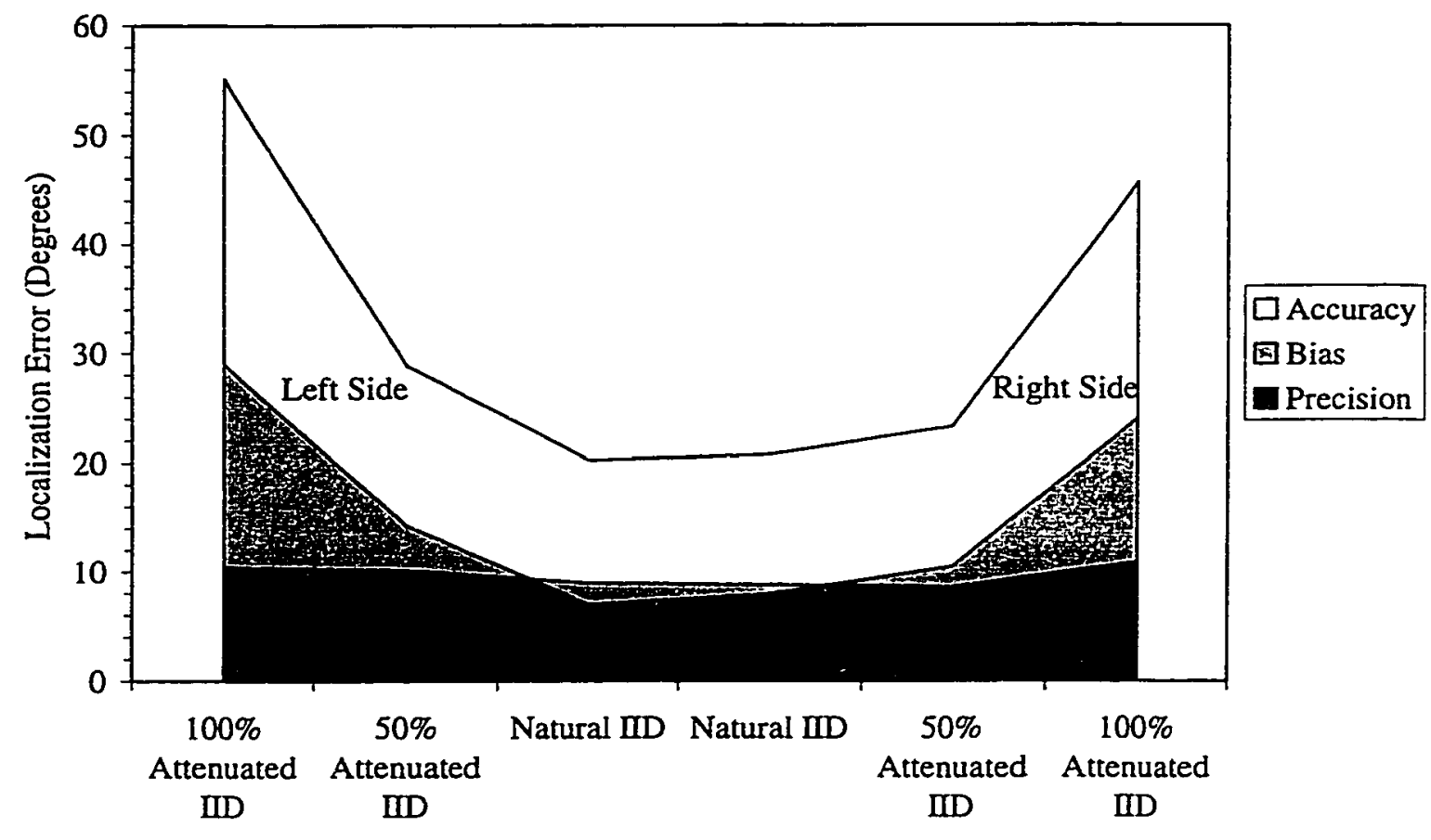

IID Condition

Figure 40: Relationship among accuracy, bias and precision of localization judgments under the three experimental interaural intensity difference conditions for the third-octave band signals. The sign of the bias on the right side has been reversed for illustrative purposes.

This figure suggests that the decrease in horizontal localization accuracy observed in this experiment for signals from which interaural intensity differences have been attenuated by $100 \%$ can be largely accounted for by bias toward the center. However, 
there was no reduction of localization accuracy (or bias) for signals in which interaural intensity differences have been attenuated to $50 \%$ of their original value in $\mathrm{dB}$. Thus, Hypothesis \#1 was supported by the results of this experiment for signals with $100 \%$ attenuated interaural intensity differences, but was not supported for signals with 50\% attenuated interaural intensity differences.

\subsubsection{Experimental Hypothesis \#2}

Hypothesis \#2 states, "For third-octave band stimuli in which interaural intensity differences have been attenuated by $50 \%$ or $100 \%$ of their original $\mathrm{dB}$ level, the magnitude of localization errors will increase with increasing center frequency."

For third-octave bands of noise, there was a significant interaction between the center frequency of the noise band and interaural intensity difference condition. This interaction is illustrated in Figure 15, above (Results, Chapter 4). There was no effect of $100 \%$ interaural intensity difference attenuation for the $0.5 \mathrm{kHz}$ noise band. In general, as center frequency increased beyond $0.5 \mathrm{kHz}$, the magnitude of the localization error also increased up to a frequency of $2.0 \mathrm{kHz}$. To this extent, the observations of this experiment support Hypothesis \#2. However, the magnitude of the localization error for the $4.0 \mathrm{kHz}$ noise band was significantly smaller than for the $2.0 \mathrm{kHz}$ noise band, indicating that there may be a maximum effect around $2.0 \mathrm{kHz}$. As noted earlier when discussing the localization of the unattenuated signals (Section 4.1.3), Mills (1958) found that spatial resolution is poorer in the $2.0 \mathrm{kHz}$ frequency region. Based upon Mills' findings, it stands to reason that disruption of localization cues might have a greater effect around $2.0 \mathrm{kHz}$.

In summary, Hypothesis \#2 is supported to the degree that the localization of third-octave bands of $0.5 \mathrm{kHz}$ was not affected by $100 \%$ interaural intensity difference 
attenuation while localization of third-octave bands of $1.0,2.0$ and $4.0 \mathrm{kHz}$ was. On the other hand, the hypothesis does not account for the fact that the effect of $100 \%$ interaural intensity difference attenuation appears to peak at about $2.0 \mathrm{kHz}$. Furthermore, Hypothesis \#2 is unsupported with regard to third-octave bands with $50 \%$ interaural intensity difference attenuation.

An important question raised by this observation is whether the peak effect at 2.0 $\mathrm{kHz}$ is the result of bias $\left(\boldsymbol{E}_{b}\right)$ or whether the effect is a result of decreased precision (increased response variability, $\sigma_{E}$ ). Figure 41 illustrates the changes in these measures across the four third-octave bands for the condition $100 \%$ attenuated. This figure suggests that the increased error observed for the third-octave band at $2.0 \mathrm{kHz}$ is a result of bias. Further evidence for this conclusion is the fact that the interaction of interaural intensity difference with frequency was not significant for the analysis of test-retest standard deviation $\left(\sigma_{E}\right)$, a measure of precision. 


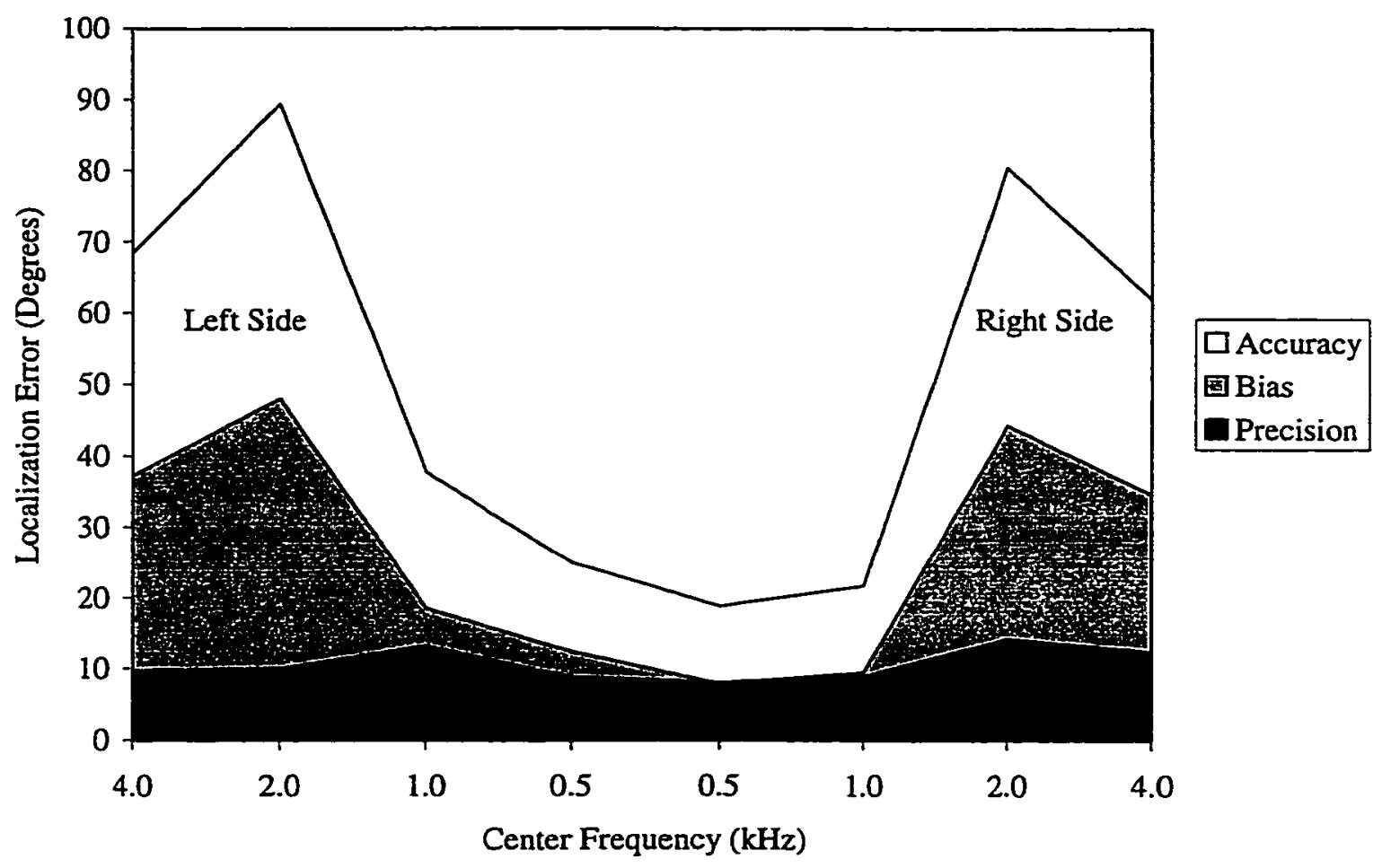

Figure 41: Contributions of precision and bias to overall accuracy in the localization of third-octave bands for the $100 \%$ attenuated interaural intensity difference condition. The sign of the bias on the right side has been reversed for illustrative purposes.

\subsubsection{Experimental Hypothesis \#3}

Hypothesis \#3 states, "For band-pass stimuli in which interaural intensity differences have been attenuated by $50 \%$ or $100 \%$ of their original $\mathrm{dB}$ level, the magnitude of localization errors will increase as the low-frequency energy content decreases."

For the band-pass noises, there was no significant interaction between signal frequency and interaural intensity difference condition for any of the three measures (accuracy, bias or precision) used in this experiment. Overall, the magnitude of the localization error was greater for the 2.0 to $4.0 \mathrm{kHz}$ band-pass noise than it was for the other band-pass noises with greater low-frequency content. However, interaural intensity 
difference attenuation resulted in a similar pattern of localization errors for all three bandpass noises, i.e., interaural intensity difference attenuation by $50 \%$ produced no effect but interaural intensity difference attenuation by $100 \%$ resulted in degraded localization performance relative to the unattenuated signal. Thus, Hypothesis \#3 is not supported by the results of this experiment.

This is in contrast to the findings of Wightman and Kistler (1992) whose subjects showed increasing effects of artificially manipulated interaural time difference cues (and decreasing reliance upon interaurai intensity difference cues) as the lower frequency content of the signals to be localized increased. $(5.0,2.5,1.0$ and $0.5 \mathrm{kHz}$ high pass cut off frequency filters).

\subsubsection{Experimental Hypothesis \#4}

Hypothesis \#4 states, "The variability of subjects' localization judgments will increase when interaural intensity difference cues are attenuated by $50 \%$ or $100 \%$ of their original $\mathrm{dB}$ value."

- For the third-octave band noises, there was no significant main effect of interaural intensity difference condition upon the test-retest standard deviation $\left(\sigma_{E}\right)$ of the localization judgments. Response variability was not affected by the elimination of interaural intensity difference cues. Thus, Hypothesis \#4 should be rejected with respect to third-octave band noises.

However, the main effect of interaural intensity difference was significant for the band-pass noises. There was an increase in test-retest variability for the $100 \%$ attenuated condition relative to the unattenuated and $50 \%$ attenuated conditions. For the band-pass noises used in this experiment, Hypothesis \#4 is supported with respect to $100 \%$ 
interaural intensity difference attenuation, but not with respect to $50 \%$ interaural intensity difference attenuation.

Examination of the interaction between interaural intensity difference condition and azimuth for the band-pass noises indicates that there was an asymmetrical effect, with the variability increasing on the left side only at the most extreme azimuths. This asymmetry was consistent across all 5 of the subjects. In order to assess the significance of the left/right asymmetry that appears in the results of the present study, a supplementary General Linear Model Repeated Measures Analysis of Variance (ANOVA) (SPSS Advanced Statistics ${ }^{\mathrm{TM}} 7.5,1997$ ) was performed separately for each signal category (third-octave band and band-pass noises) over the absolute value of the dependent variable bias $\left(\boldsymbol{E}_{b}\right)$. The absolute value was calculated in order to remove the sign of the bias, which, when averaged over the left and right sides, will tend to cancel out (positive bias on the left side and negative bias on the right side). The factors for the analysis were room (ROOM, 2 levels), signal type (FREQ, 4 levels for third-octave bands, interaural intensity difference condition (IID, 3 levels), 3 levels for band-pass noises), side (SIDE, 2 levels), and loudspeaker location (AZIM, 9 levels) with repeated measures over 5 subjects. The ANOVA tables for these analyses appear in the Appendix in Table 12 and Table 13. The factor side was significant $(p>0.05)$ for both the thirdoctave band noises and the band-pass noises. In addition, several interactions involving the factor side were significant. The three-way interaction interaural intensity difference condition by side by azimuth was significant for both the third-octave bands and the band-pass noises. The interactions interaural intensity difference condition by side and side by azimuth were significant for the band-pass noises only. Finally, a four-way 
interaction among signal type, interaural intensity difference condition, side and azimuth was significant for the third-octave bands only. These results indicate that for these subjects there was a tendency for greater bias on the left side (toward the right) than on the right side.

All subjects in this experiment were right-handed, so it is possible that there is a relationship between performance on a spatial task and the right ear advantage (Ward, 1957; Emmerich et. al, 1988). Other left-right asymmetries have been observed in the literature. For example, Grantham (1996) observed an asymmetrical advantage in the buildup of echo suppression when the lead stimulus comes from the right side versus when it comes from the left side. Grantham (1996) also discusses other hearing asymmetries that have been reported. These include higher prevalence of spontaneous otoacoustic emissions in right ears relative to left ears, higher wave $I I$ and $I I I$ amplitudes of the human auditory brainstem response for right ear relative to left ear presentations, ear preference for speech or melodies in dichotic tasks and ear dominance for pitch.

An asymmetrical effect in auditory localization was also described by Hartmann (1983). In that experiment, there was an exclusively leftward bias for 7 of 12 subjects and an exclusively rightward bias for 1 of the 12 subjects. Although further data were collected in an attempt to determine if the biases had an acoustical or a psychological origin, no satisfactory answer could be determined. The issue of asymmetrical biases in auditory localization experiments would seem to be an interesting area for further research.

\subsubsection{Experimental Hypotheses \#5 and \#6}

Hypothesis \#4 states, "The localization of signals recorded in a reverberant environment will be more variable than for those recorded in an anechoic chamber." 
Hypothesis \#5 states, "The localization of signals recorded in a reverberant environment will be less affected by the attenuation of interaural intensity differences than those recorded in an anechoic chamber."

There was no significant main effect of room in the analysis of variance that was performed on the test-retest standard deviation of the localization responses. Subjects localized signals recorded in the reverberant room with as much precision as they did those recorded in the anechoic room. Nor was there a significant difference in the accuracy with which subjects localized sounds that were recorded in the reverberant versus the anechoic room rooms used in this experiment under any of the interaural intensity difference conditions. Attenuation of interaural intensity difference resulted in identical effects for both rooms.

The reason that the room in which the signals were recorded made no difference in the ability of the subjects to localize the sound sources is probably related to the characteristics of the reverberant room that was used. The room was quite large (337.57 $\mathrm{m}^{3}$ in volume) with a reverberation time of only about $400 \mathrm{msec}$. The loudspeaker was located 1 meter from KEMAR, which was well within the critical distance of approximately 5.8 meters. Thus, the preponderance of the recorded sound energy came from the direct field rather than the reverberant field. Thus, this experiment proved not to be a good test of Hypothesis \#5 and Hypothesis \#6. Neither hypothesis was supported by its results.

\subsection{Implications for spatial hearing with binaural hearing aids}

Binaural hearing aids are often fit on hard-of-hearing individuals by audiologists who are concerned about their clients' sense of auditory space. A number of studies have shown that horizontal auditory localization cues are preserved by the use of binaural 
hearing aids (Byrne \& Dermody, 1975; Markides, 1977; Noble \& Byrne, 1987) and that hard-of-hearing listeners generally perform better with binaural than monaural hearing aid fittings. These results are logical considering that interaural time difference and interaural intensity difference cues are likely to be preserved in a binaural fitting of matched linear ear-level hearing aids as long as the amplitude of the signals is within the dynamic range of the hearing aid and the hearing aid is not saturated.

However, many modern hearing aids use amplitude compression in an attempt to limit the loudness of amplified signals without creating audible distortion (as occurs with peak clipping) or to accommodate for recruitment in the ears of hard-of-hearing listeners. In cases where the amplitude compression scheme is designed to limit high level output, there is little concern that interaural time difference and interaural intensity difference cues will be affected for signals within a wide dynamic range, except in cases of severe hearing loss where the dynamic range is small. In loudness-based hearing aid algorithms in which wide-dynamic range compression is used, it is possible that interaural intensity difference cues will be degraded for a person using binaural hearing aids because the intensity of the hearing aid output is controlled independently in each hearing aid. The difference in the level of the sound arriving at each head-worn hearing aid microphone will be reduced in cases where amplitude compression circuits are used to adjust the level of sounds to fit into a given dynamic range for the listener.

The results of the present study indicate that a wide dynamic range compression hearing aid will not adversely affect horizontal localization for listeners in which the compression ratio is $2: 1$ or less. A 2:1 compression ratio means that the input level has to increase $2 \mathrm{~dB}$ in order for the output level to increase $1 \mathrm{~dB}$. Thus, if the sound arriving at 
the left microphone is $10 \mathrm{~dB}$ greater in level than that arriving at the right microphone (10 dB interaural intensity difference), a 2:1 compression circuit will adjust the levels such that the difference between the ears becomes $5 \mathrm{~dB}$. This, of course, assumes that both the right and left input signals are above the level of the compression threshold. Scenarios can be imagined in which the signal at one ear is above, and the signal in the opposite ear is below the threshold of compression. In these situations, the ID may be adjusted by less than would be predicted by the compression ratio. Since reduction of the interaural intensity difference by $50 \%$ in this experiment did not result in disruptions of horizontal auditory localization, it is not likely that a compression ratio of $2: 1$ would adversely affect localization either. On the other hand, if the compression ratio were greater than $2: 1$, the results of this experiment suggest that it is possible that interaural intensity difference adjustments would be great enough to result in the disruption of localization.

Another factor that might come into play in a binaural compression hearing aid fit is the attack time of the compression circuit. If the attack time were longer than the integration time of the ear, the arriving sound may be localized differently than if a very rapid attack time were in use. It is possible that an effect similar to the precedence effect (Haas, 1951) would occur and that the earliest cues would be the effective ones. On the other hand, it may be that the apparent location of the sound source would shift as the compression circuit was activated. No such effect has been documented in wearers of binaural wide dynamic range compression hearing aids. It is hoped that further research on the horizontal auditory localization of sounds heard through hearing aids that are using compression circuits will help sort out these issues and document the effect of such circuits on binaural hearing. 


\subsection{Conclusions}

Generally, the overall localization accuracy of the subjects in this experiment was similar to that reported in the literature. Localization accuracy was greater in the front than at the sides, consistent with Mills (1958) and Makous et al. (1990) but not consistent with Oldfield and Parker (1984) or Wightman and Kistler (1989). In addition, there was an artifactual end effect in the data that has been observed in other experiments in which the response area is restricted, e.g., Butler, et at. (1990) and Giguère and Abel (1993).

The major finding of this experiment is that for narrow bands of noise, attenuating interaural intensity differences by $100 \%$ degraded localization performance principally by introducing a bias toward the center. Auditory localization performance was not degraded by reducing interaural intensity difference cues to $50 \%$ of their original value. The exception to this general conclusion is the third-octave band of noise with a center frequency of $0.5 \mathrm{kHz}$. The localization of this signal was not degraded by $100 \%$ attenuation of interaural intensity differences. These findings are consistent with the theory that interaural time differences will dominate auditory localization in conflicting cue experiments unless interaural intensity difference manipulations exceed about $10 \mathrm{~dB}$. This hypothesis is also generally supported by the findings of Hartmann and Fontana (1991).

In addition to introducing an bias, reduction of interaural intensity differences to zero resulted in a decrement in the precision of localization (as measured by the testretest standard deviation) for signals presented on the far left of the listeners. This asymmetric phenomenon is unexplained, however other asymmetrical effects have been reported in the auditory localization literature and may present an opportunity for further research. 
In contrast to the findings of Giguère and Abel (1993), there was no degradation in localization performance when the binaural signals were recorded in a relatively reverberant room relative to those recorded in an anechoic chamber. This result may be due to the size of the room, the relatively short $(400 \mathrm{~ms})$ reverberation time and the distance of the loudspeaker from KEMAR.

The results of this research suggest that wide dynamic range compression hearing aids worn binaurally are not likely to have a deleterious effect upon users' auditory localization performance. This is an important issue for hearing aid users who rely heavily upon spatial cues, such as those with impaired vision. Further research into the effects of amplitude compression upon spatial hearing is needed. 


\section{CHAPTER 6: APPENDIX}

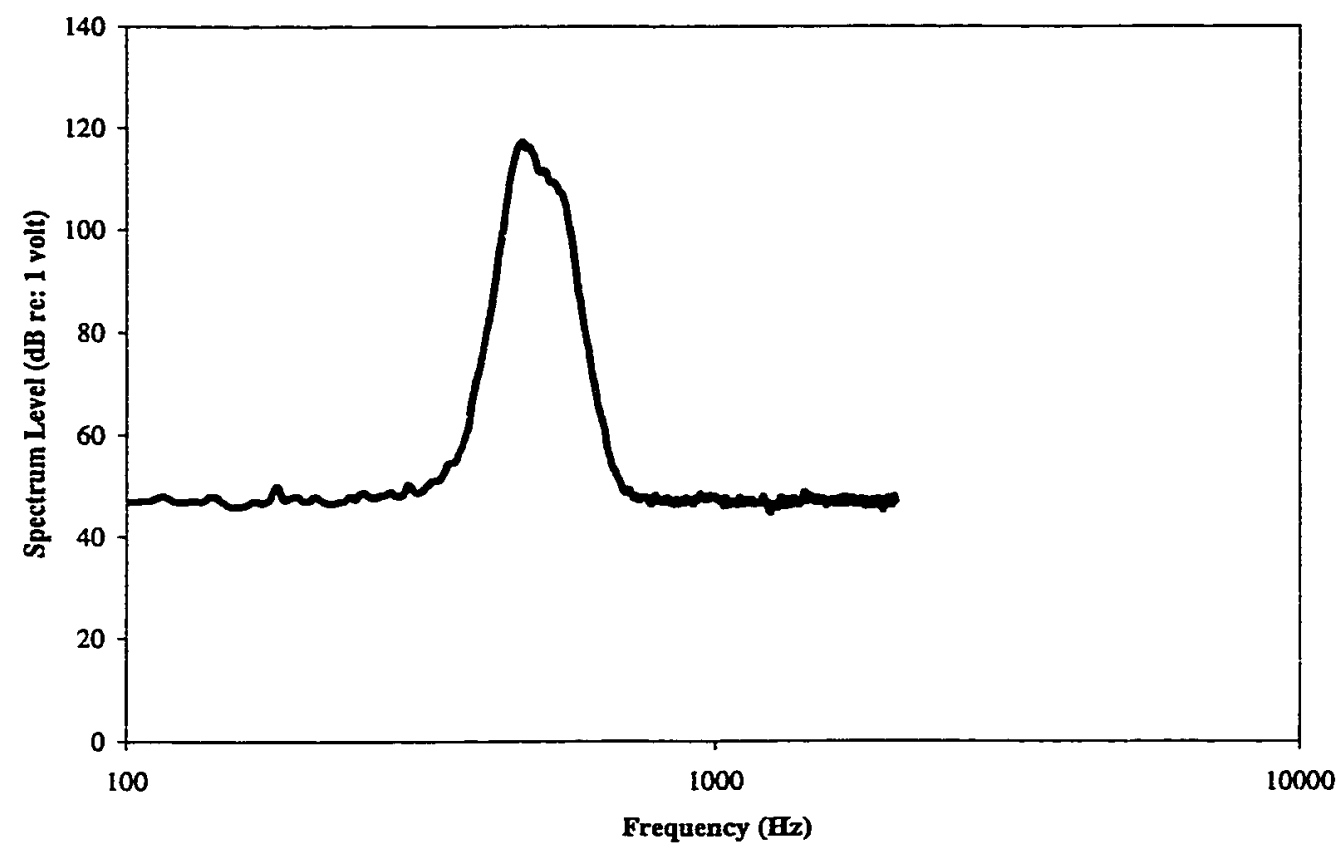

Figure 42: Spectrum of the $0.5 \mathrm{kHz}$ third-octave band noise used in the present experiment.

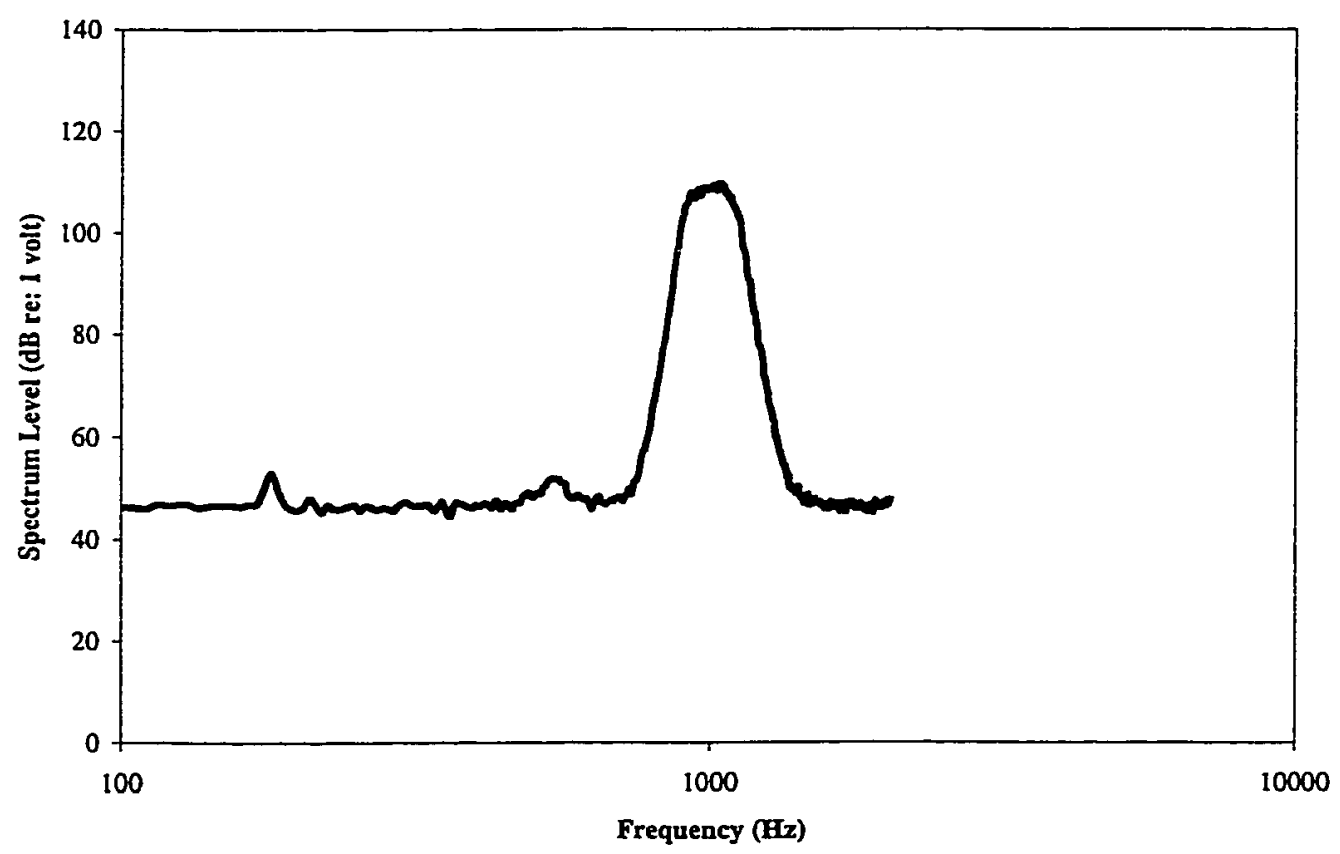

Figure 43: Spectrum of the $1.0 \mathrm{kHz}$ third-octave band noise used in the present experiment. 


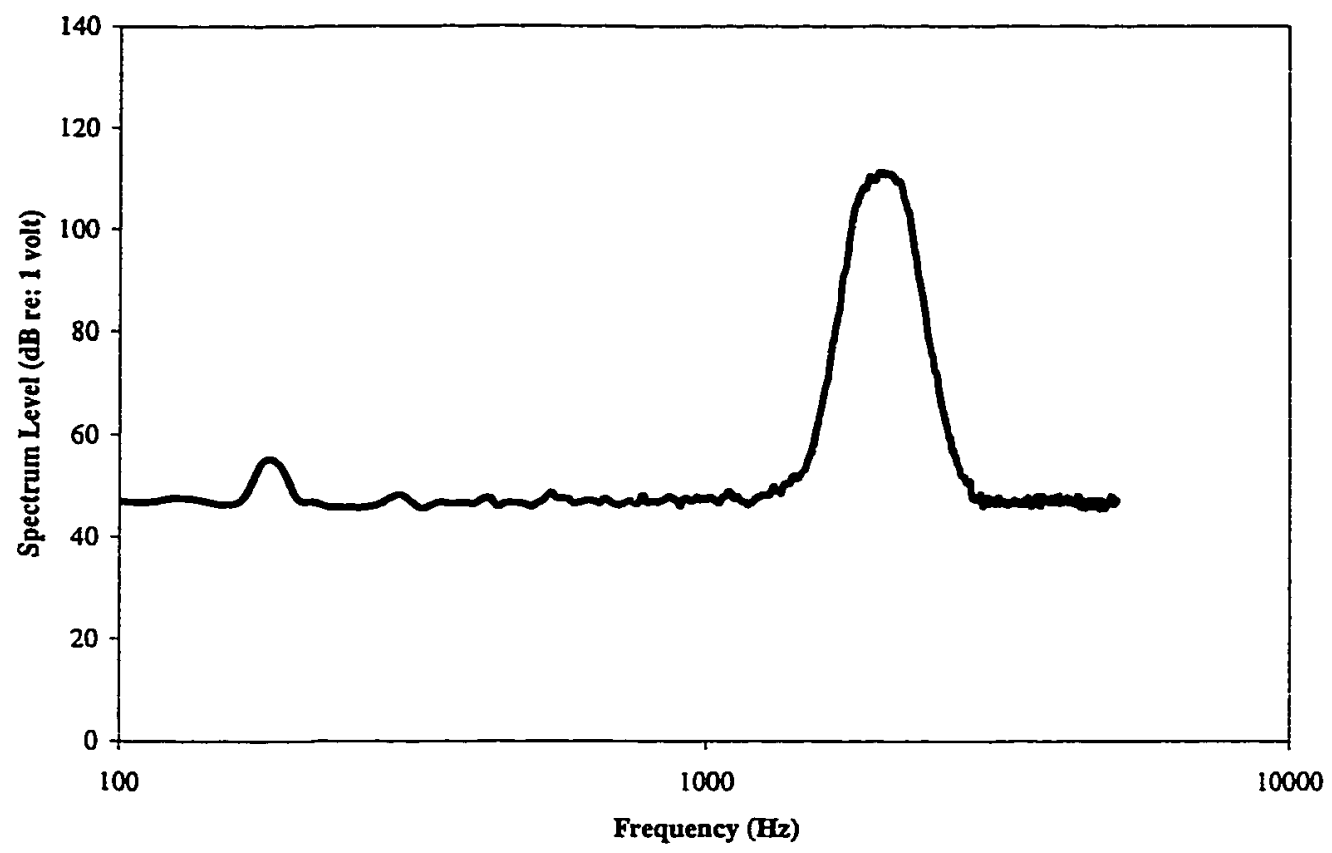

Figure 44: Spectrum of the $2.0 \mathrm{kHz}$ third-octave band noise used in the present experiment.

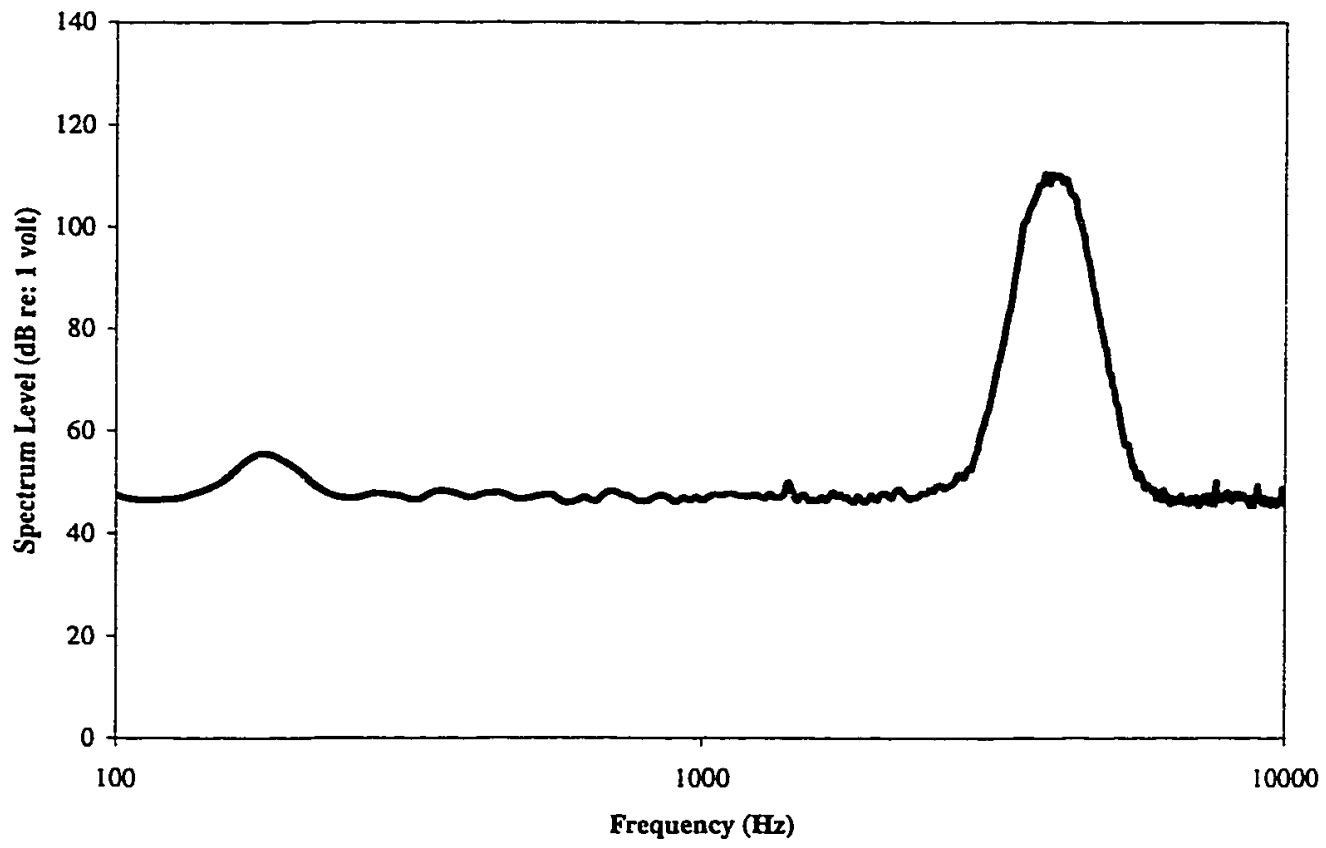

Figure 45: Spectrum of the $4.0 \mathrm{kHz}$ third-octave band noise used in the present experiment. 


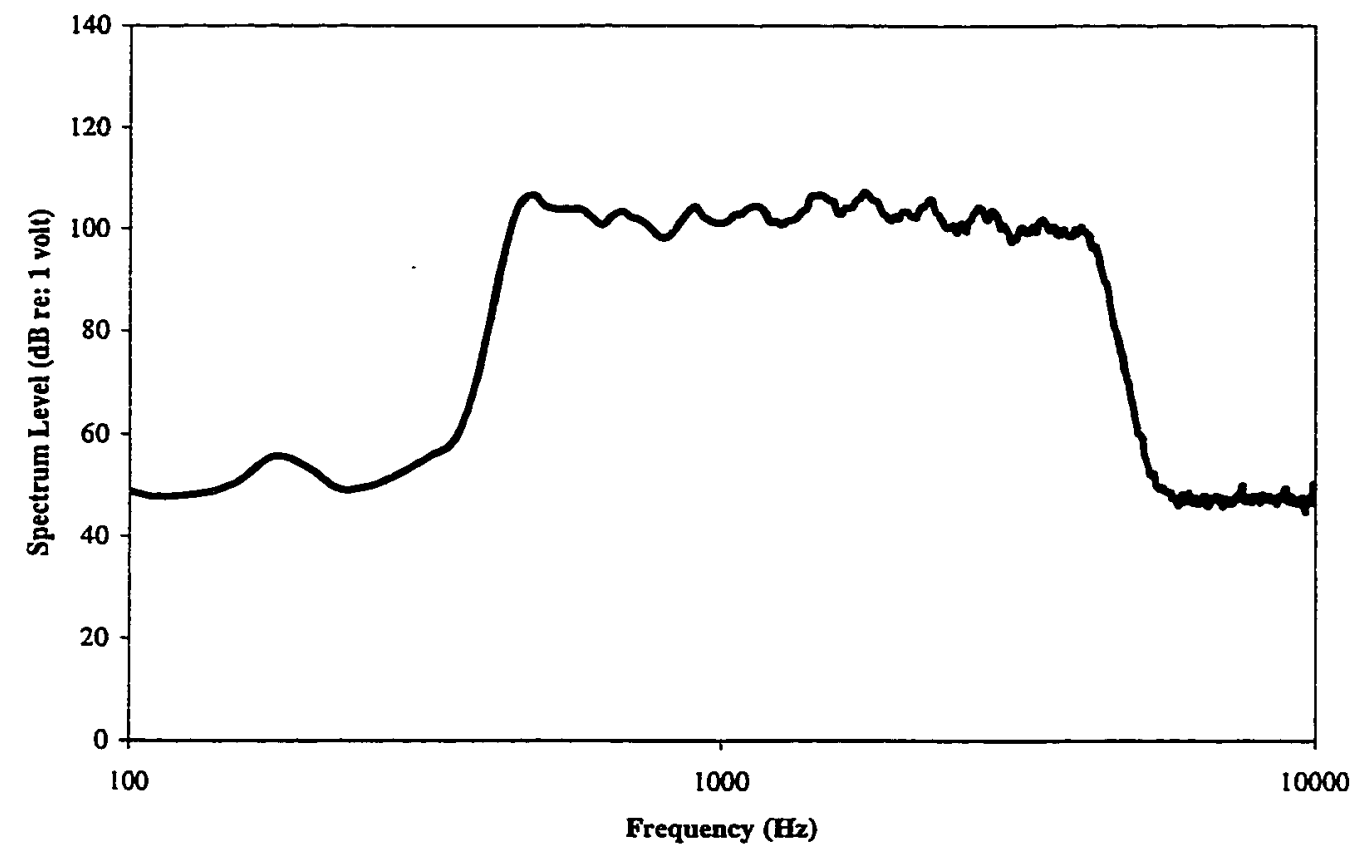

Figure 46: Spectrum of the $0.5 \mathrm{kHz}$ to $4.0 \mathrm{kHz}$ band-pass noise used in the present experiment.

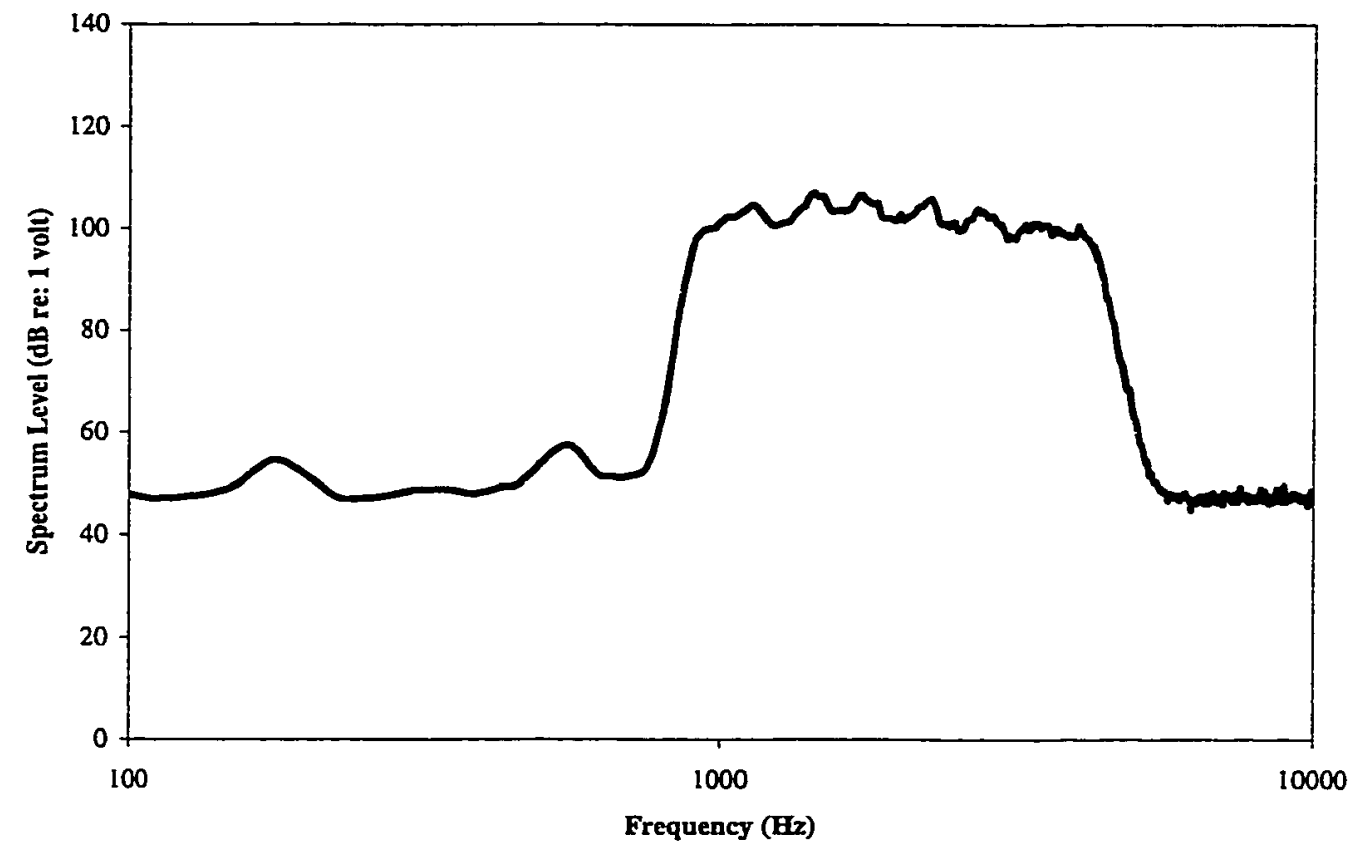

Figure 47: Spectrum of the 1.0 to $4.0 \mathrm{kHz}$ band-pass noise used in the present experiment. 


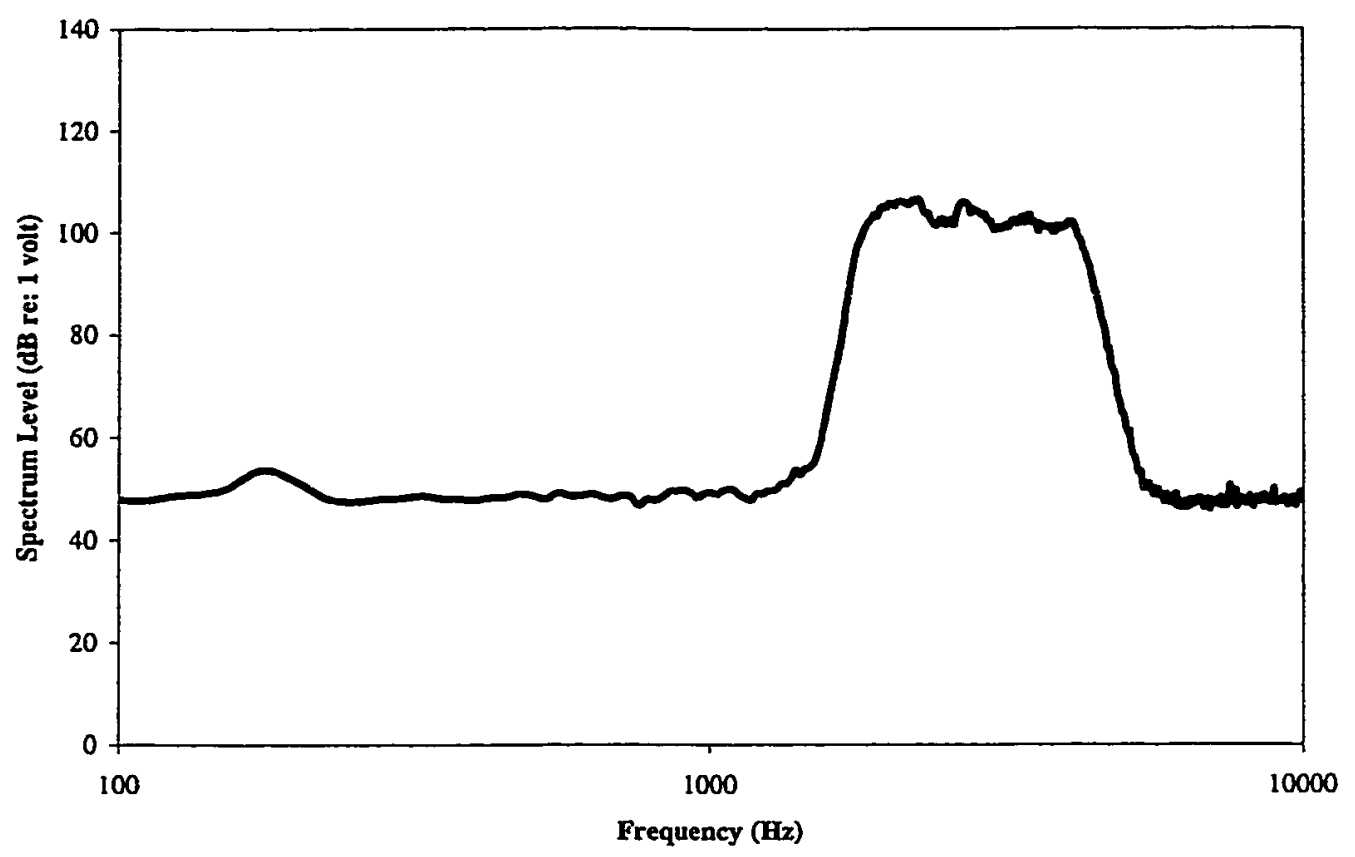

Figure 48: Spectrum of the $2.0 \mathrm{kHz}$ to $4.0 \mathrm{kHz}$ band-pass noise used in the present experiment.

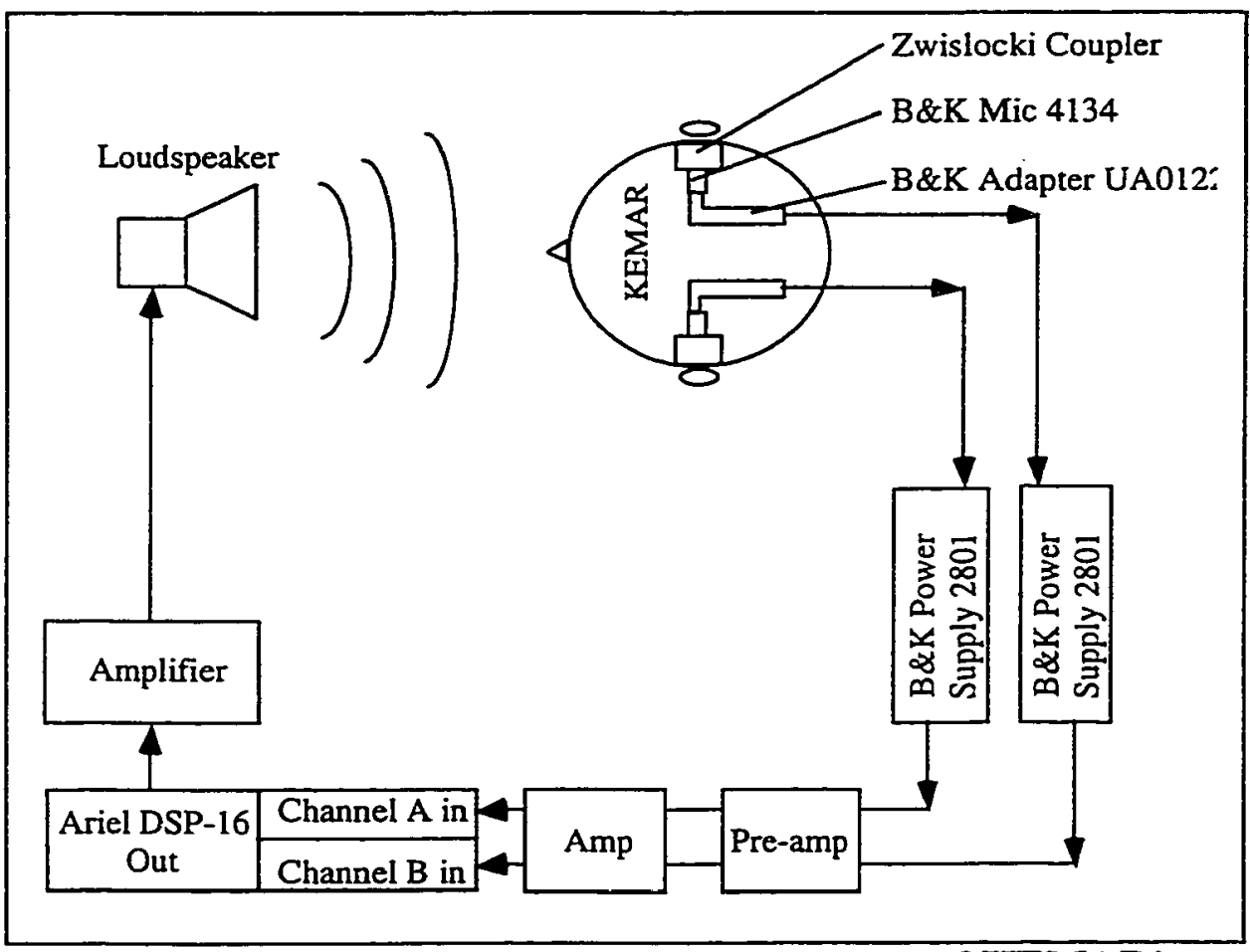

Figure 49: Instrumentation for measuring the response of KEMAR's ears to a broad band stimulus at each loudspeaker location. 


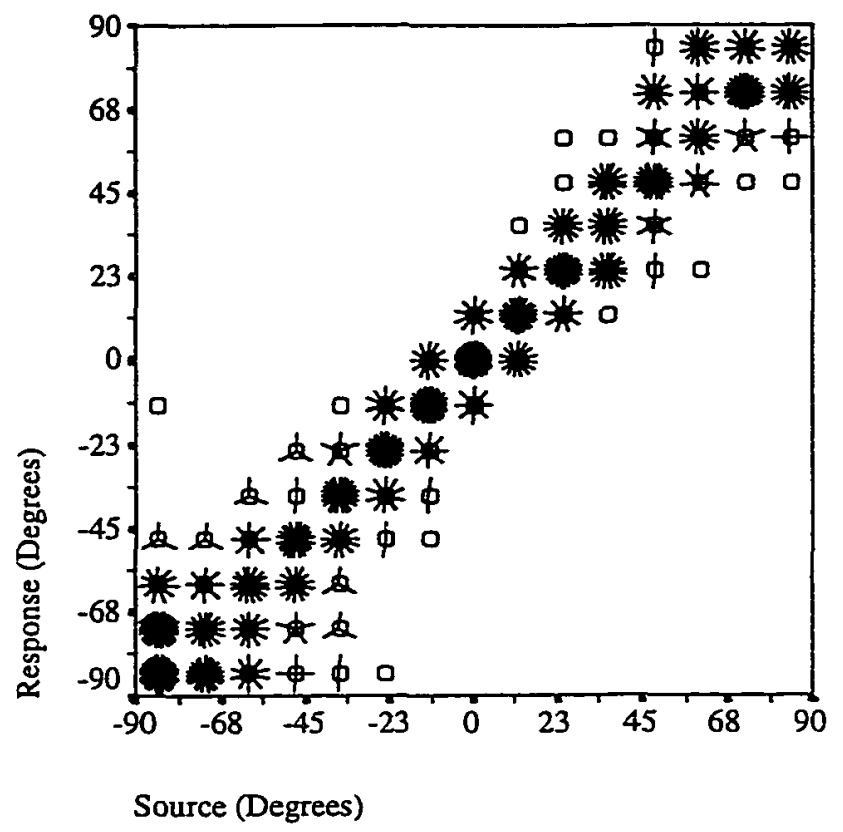

Figure 50: $0.5 \mathrm{kHz}$ third-octave band noise with interaural intensity differences unattenuated.

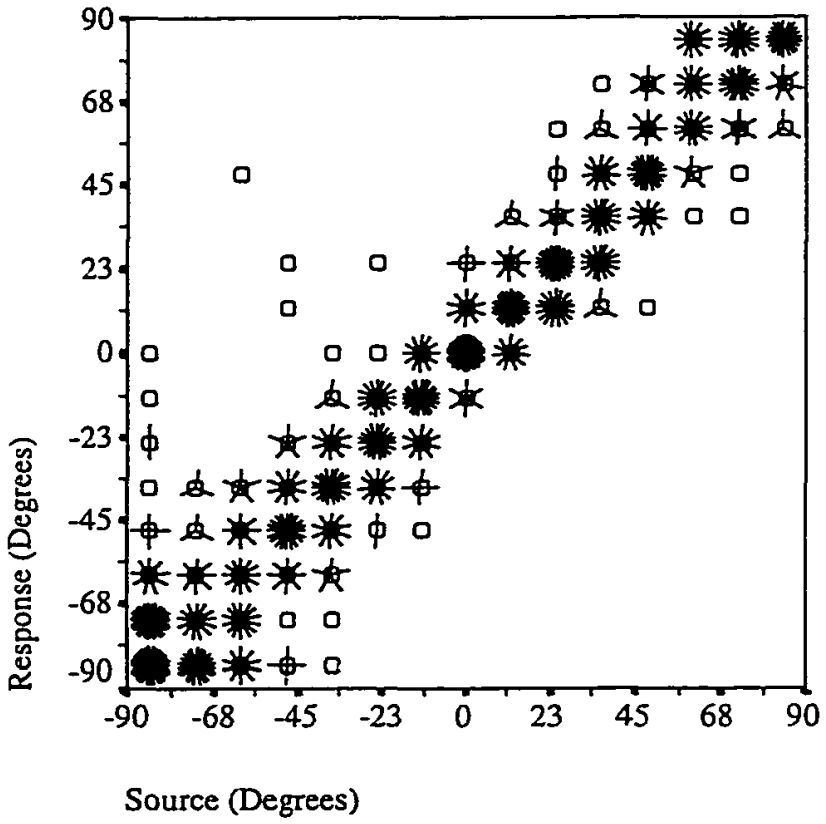

Figure 51: $0.5 \mathrm{kHz}$ third-octave band noise with interaural intensity differences attenuated by $50 \%$. 


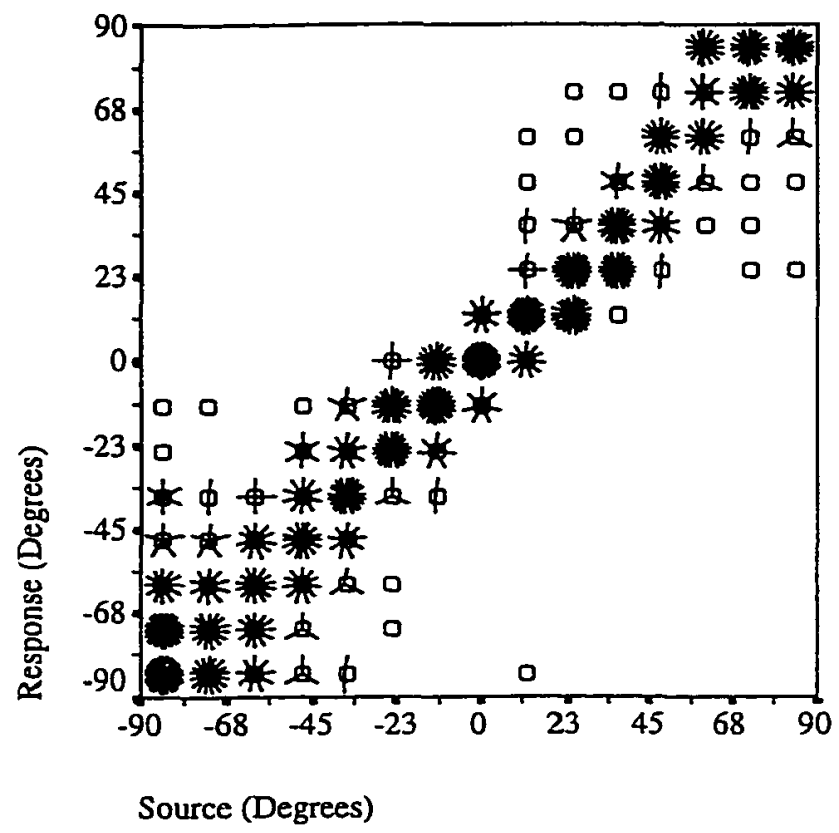

Figure 52: $0.5 \mathrm{kHz}$ third-octave band noise with interaural intensity differences attenuated by $100 \%$.

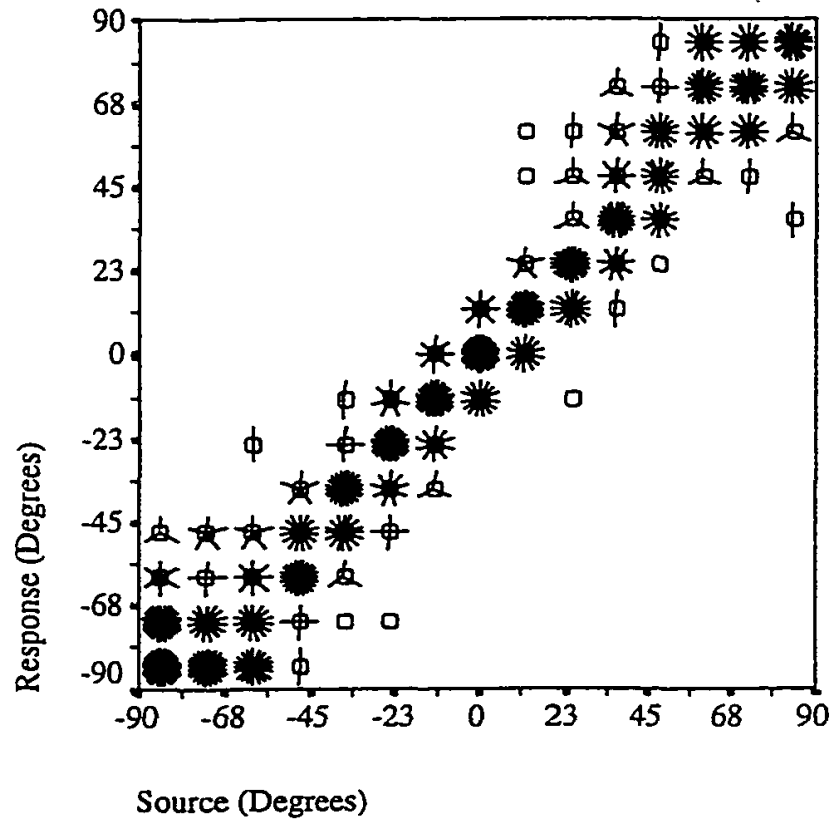

Figure 53: $1.0 \mathrm{kHz}$ third-octave band noise with interaural intensity differences unattenuated. 


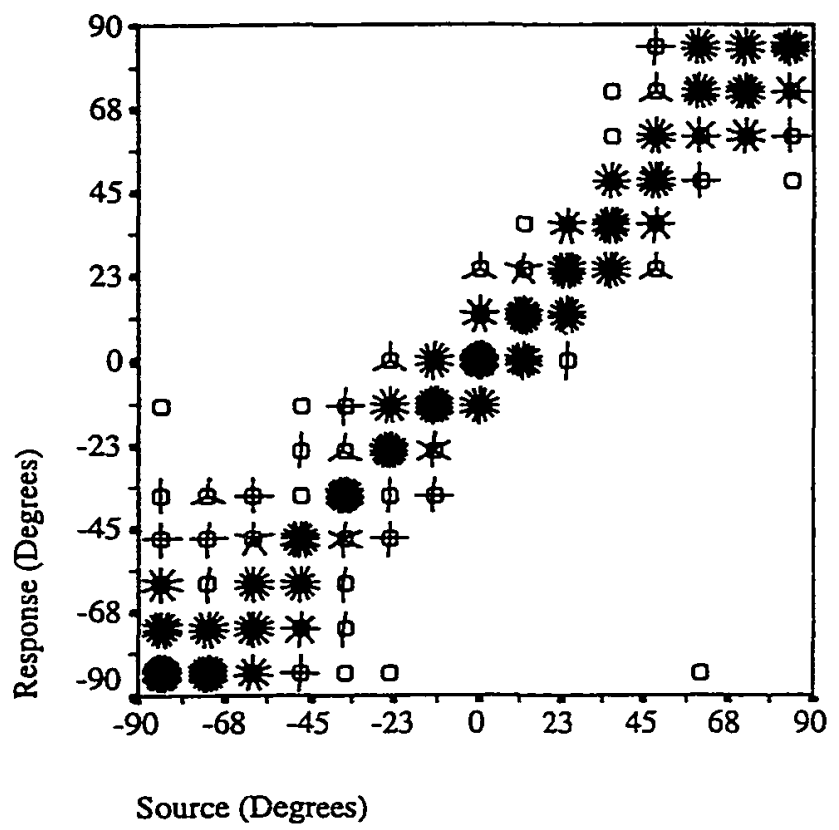

Figure 54: $1.0 \mathrm{kHz}$ third-octave band noise with interaural intensity differences attenuated by $50 \%$.

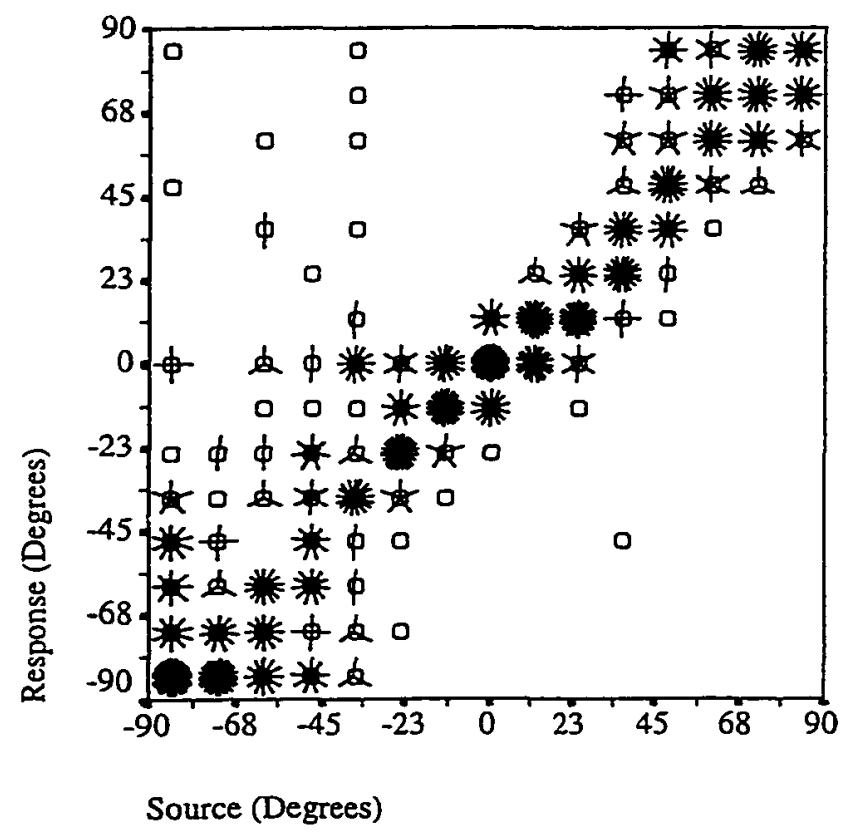

Figure 55: $1.0 \mathrm{kHz}$ third-octave band noise with interaural intensity differences attenuated by $100 \%$. 


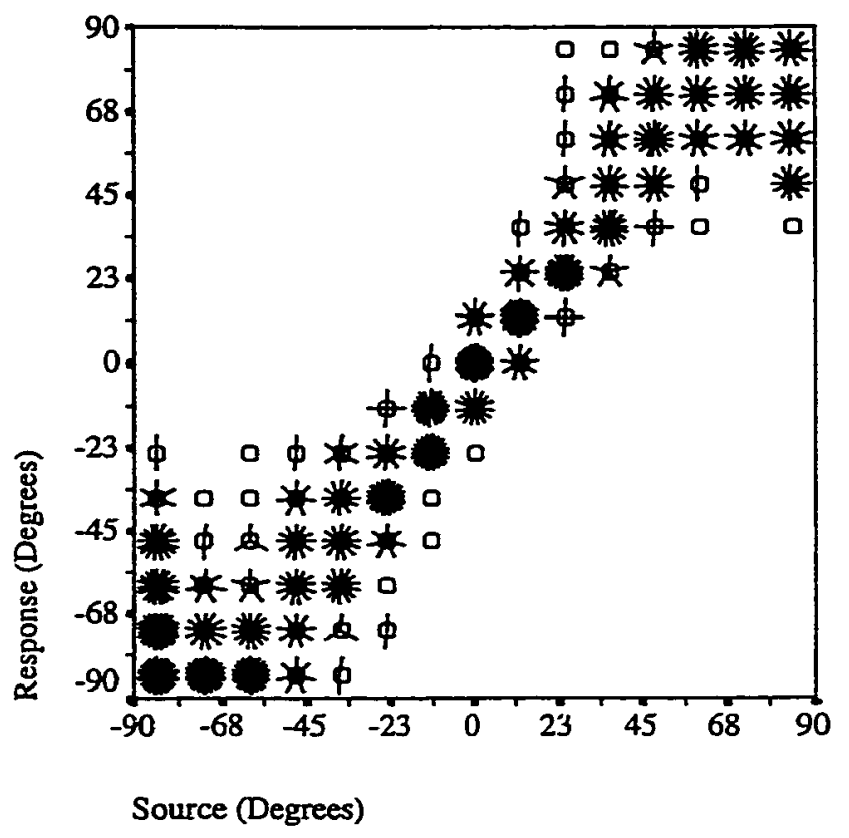

Figure 56: $2.0 \mathrm{kHz}$ third-octave band noise with interaural intensity differences unattenuated.

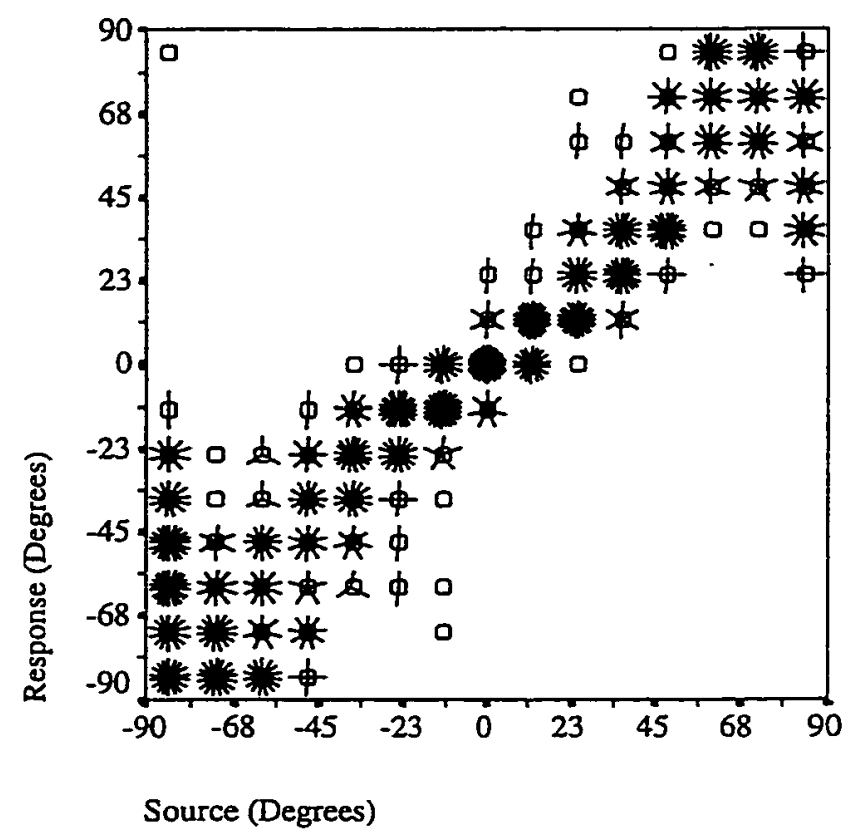

Figure 57: $2.0 \mathrm{kHz}$ third-octave band noise with interaural intensity differences attenuated by $50 \%$. 


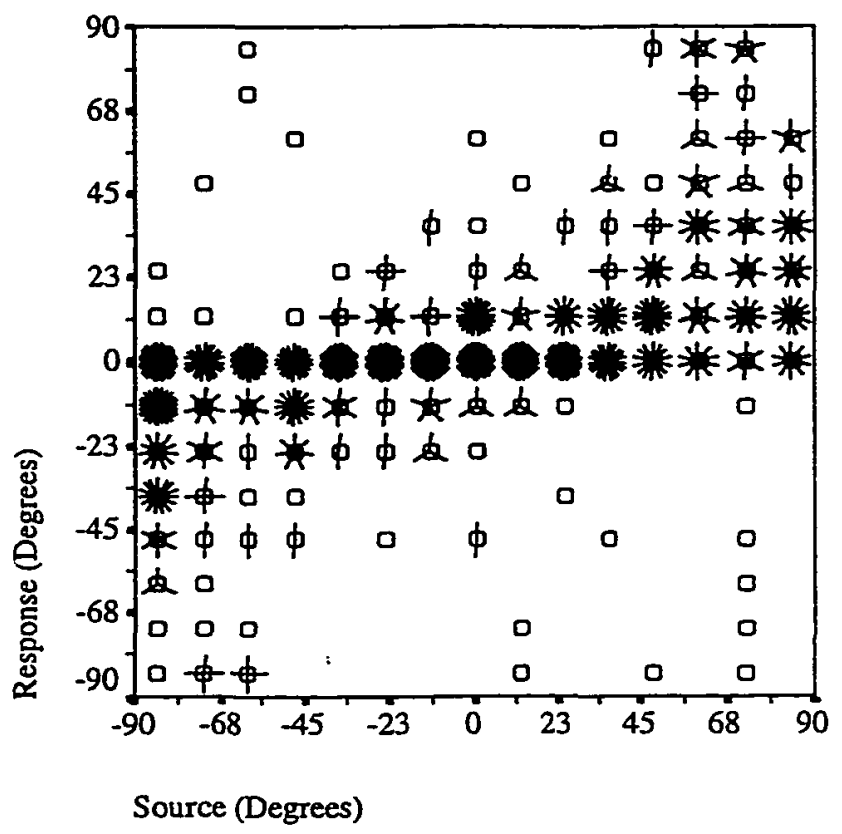

Figure 58: $2.0 \mathrm{kHz}$ third-octave band noise with interaural intensity differences attenuated by $100 \%$.

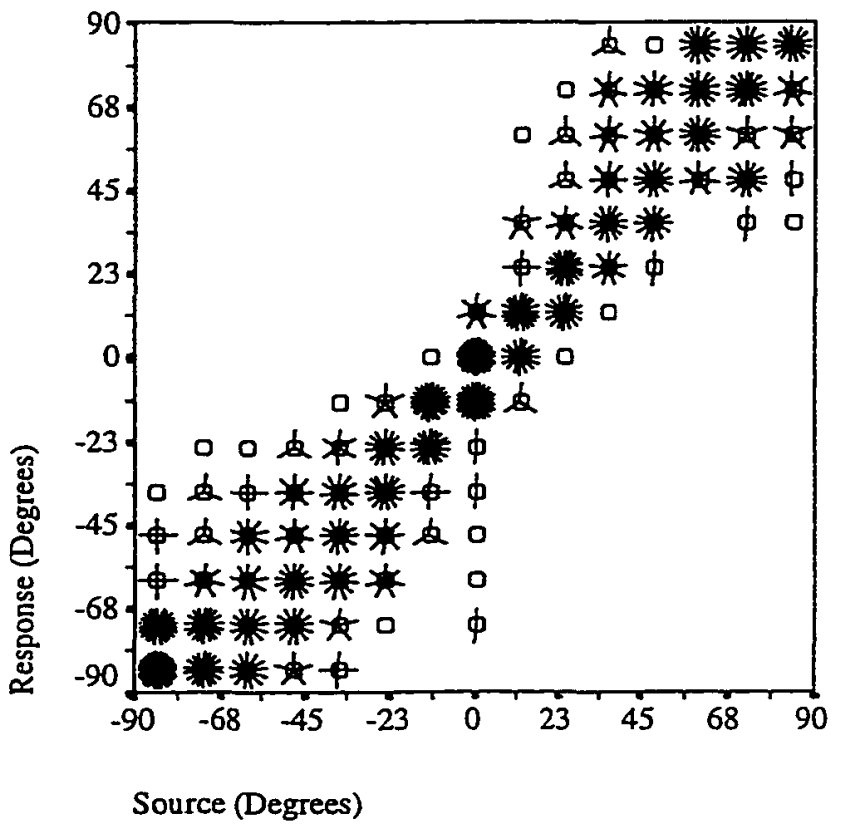

Figure 59: $4.0 \mathrm{kHz}$ third-octave band noise with interaural intensity differences unattenuated. 


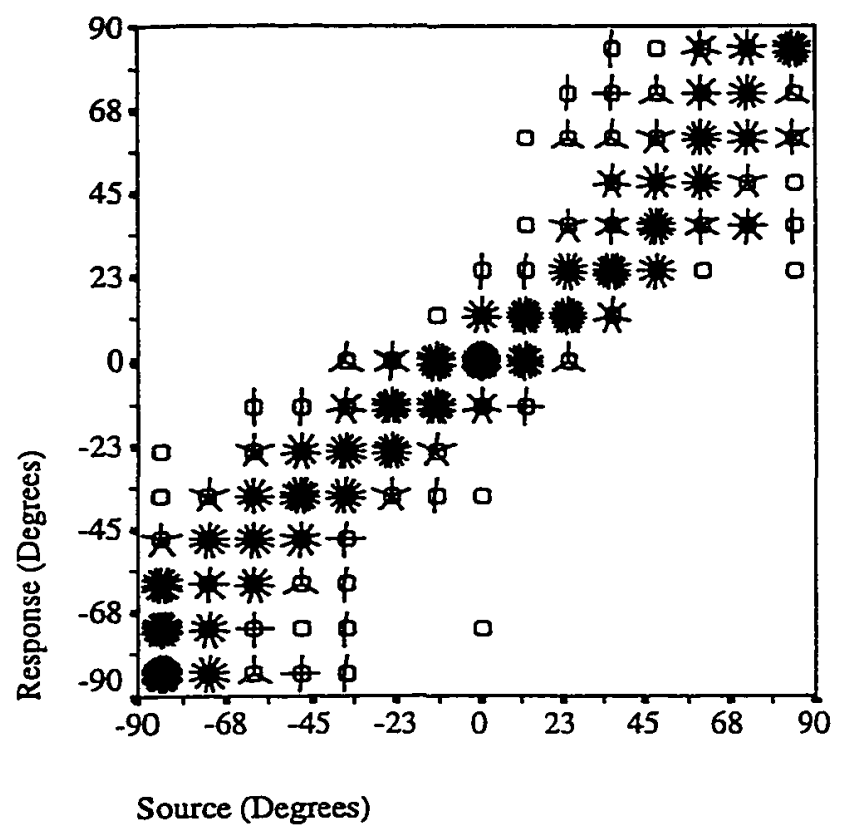

Figure 60: 4.0 kHz third-octave band noise with interaural intensity differences attenuated by $50 \%$.

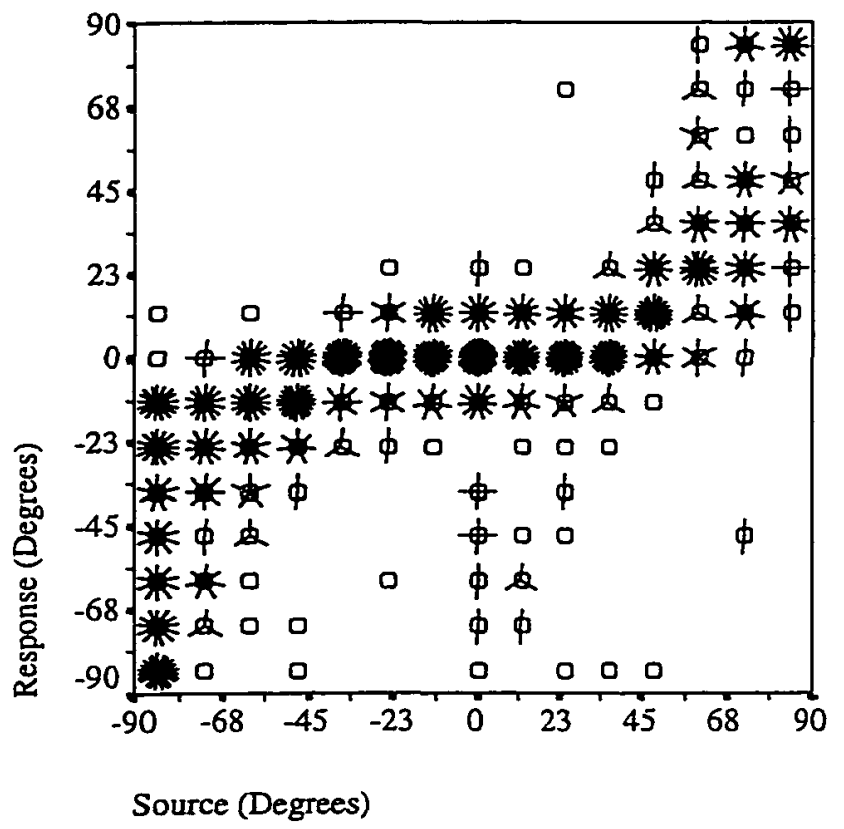

Figure $61: 4.0 \mathrm{kHz}$ third-octave band noise with interaural intensity differences attenuated by $100 \%$. 


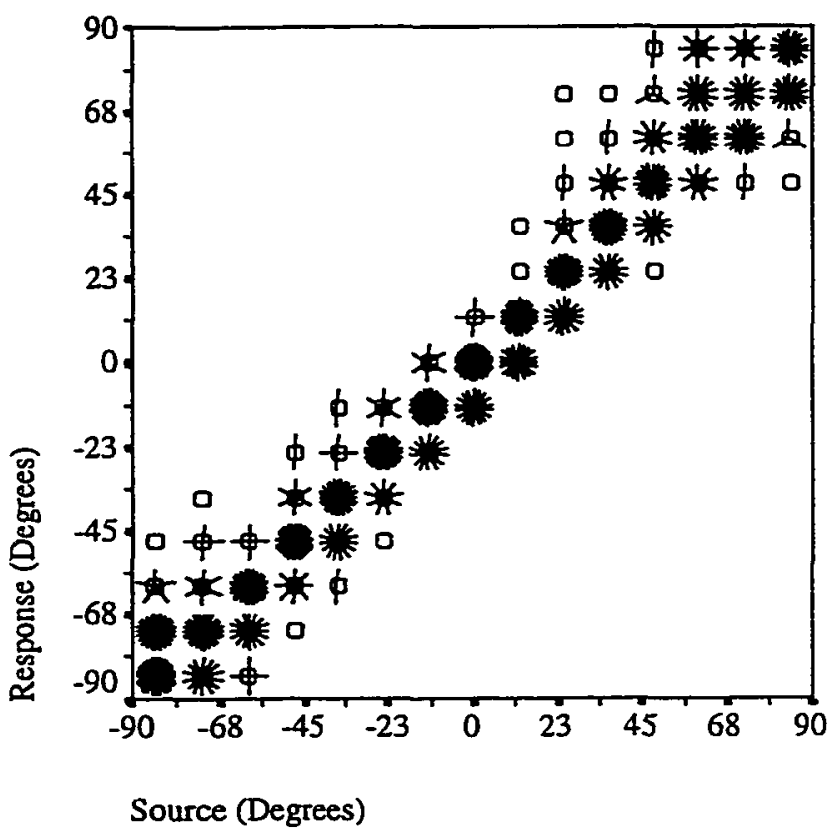

Figure 62: $0.5 \mathrm{kHz}$ to $4 \mathrm{kHz}$ band-pass noise with interaural intensity differences unattenuated.

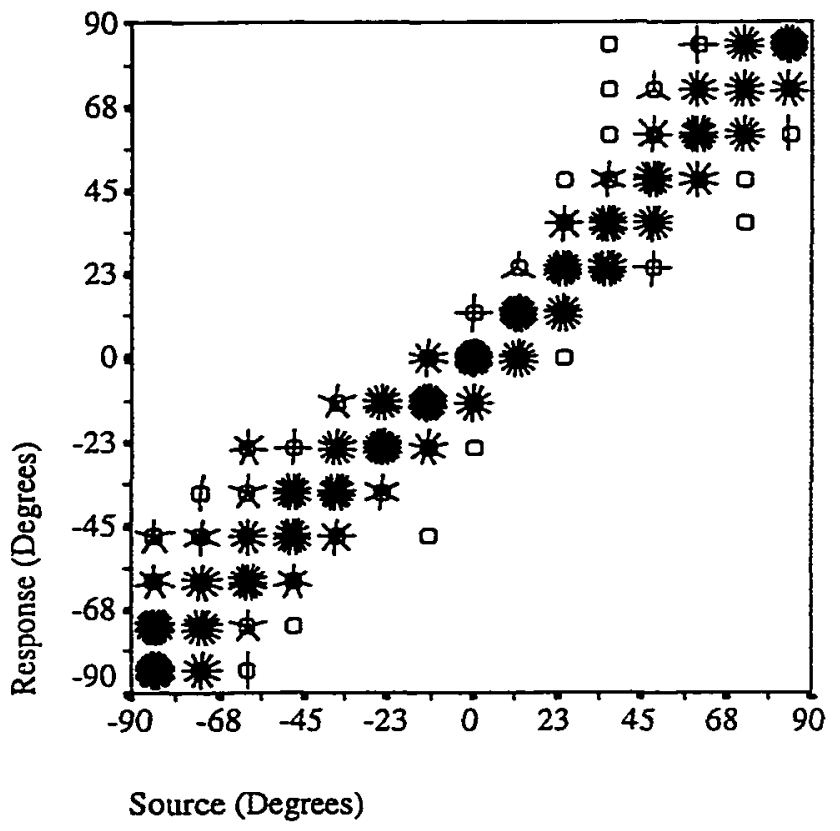

Figure 63: $0.5 \mathrm{kHz}$ to $4 \mathrm{kHz}$ band-pass noise with interaural intensity differences attenuated by $50 \%$. 


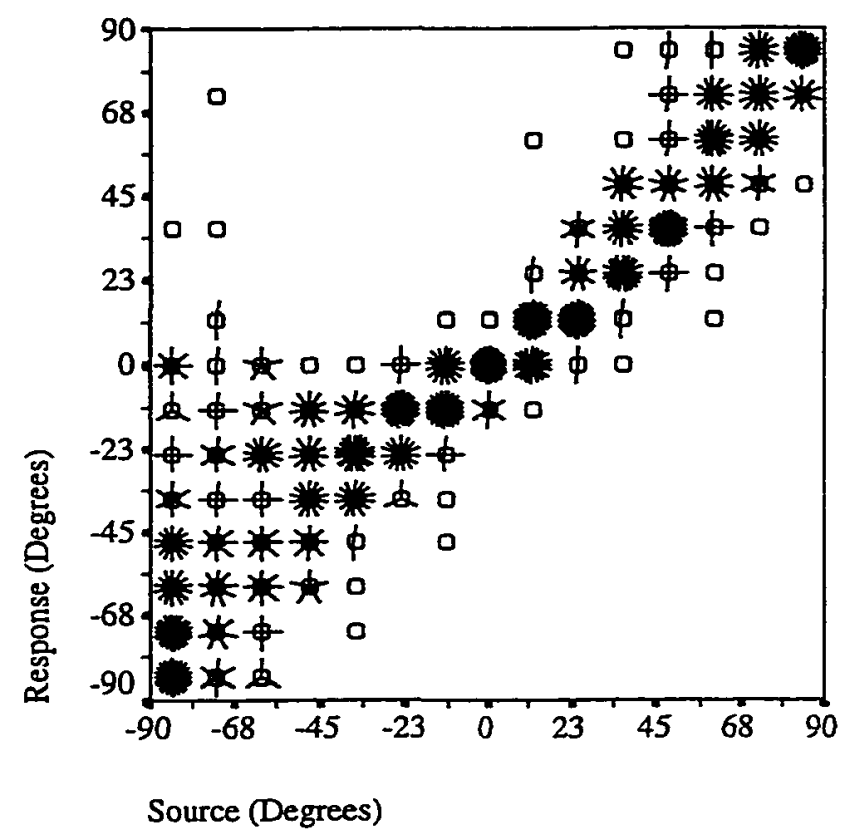

Figure 64: $0.5 \mathrm{kHz}$ to $4 \mathrm{kHz}$ band-pass noise with interaural intensity differences attenuated by $100 \%$.

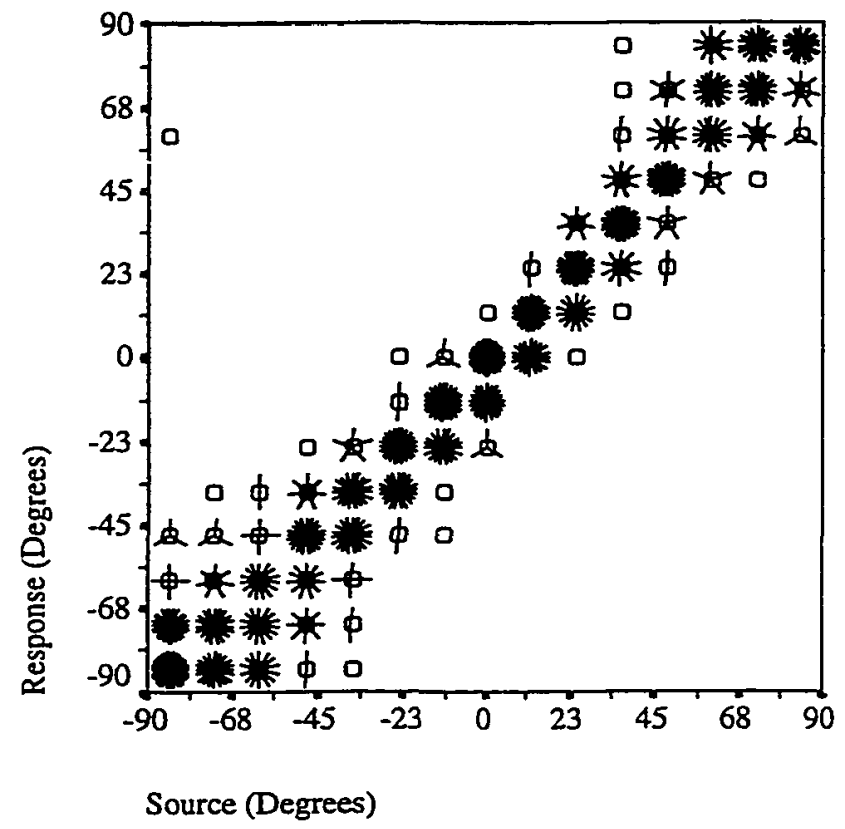

Figure 65: $1.0 \mathrm{kHz}$ to $4.0 \mathrm{kHz}$ band-pass noise with interaural intensity differences unattenuated. 


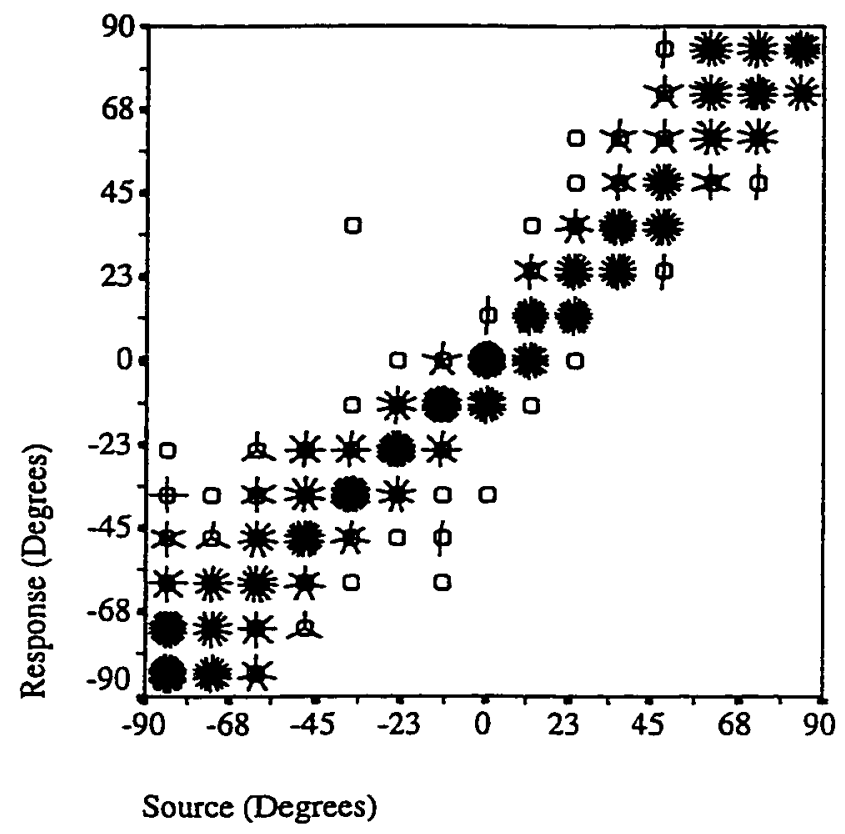

Figure 66: $1.0 \mathrm{kHz}$ to $4.0 \mathrm{kHz}$ band-pass noise with interaural intensity differences attenuated by $50 \%$.

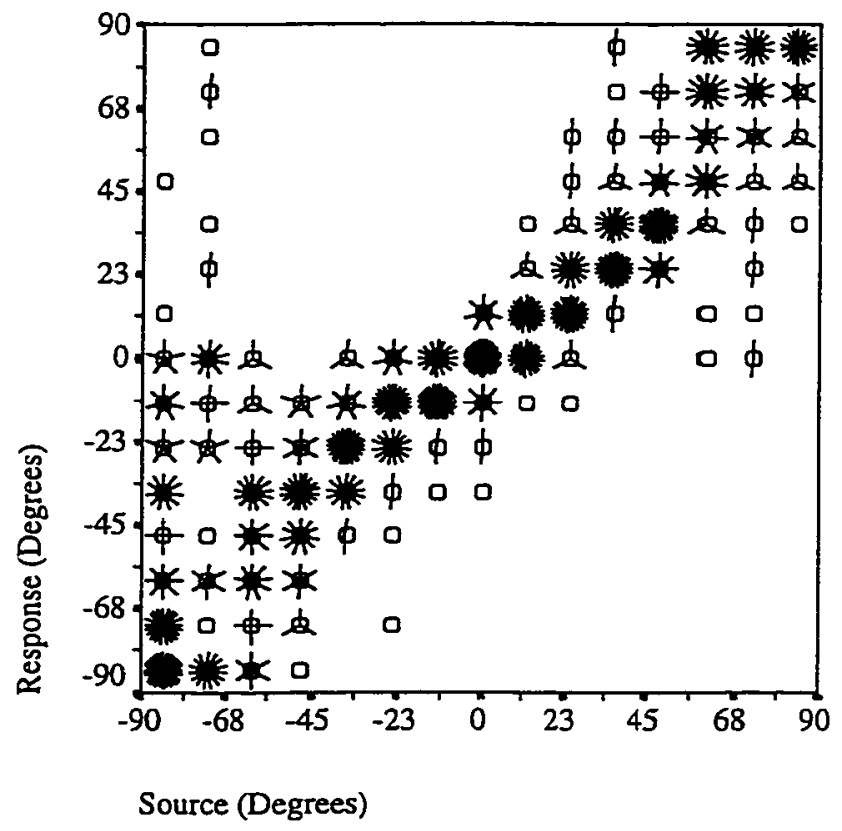

Figure 67: $1.0 \mathrm{kHz}$ to $4.0 \mathrm{kHz}$ band-pass noise with interaural intensity differences attenuated by $100 \%$. 


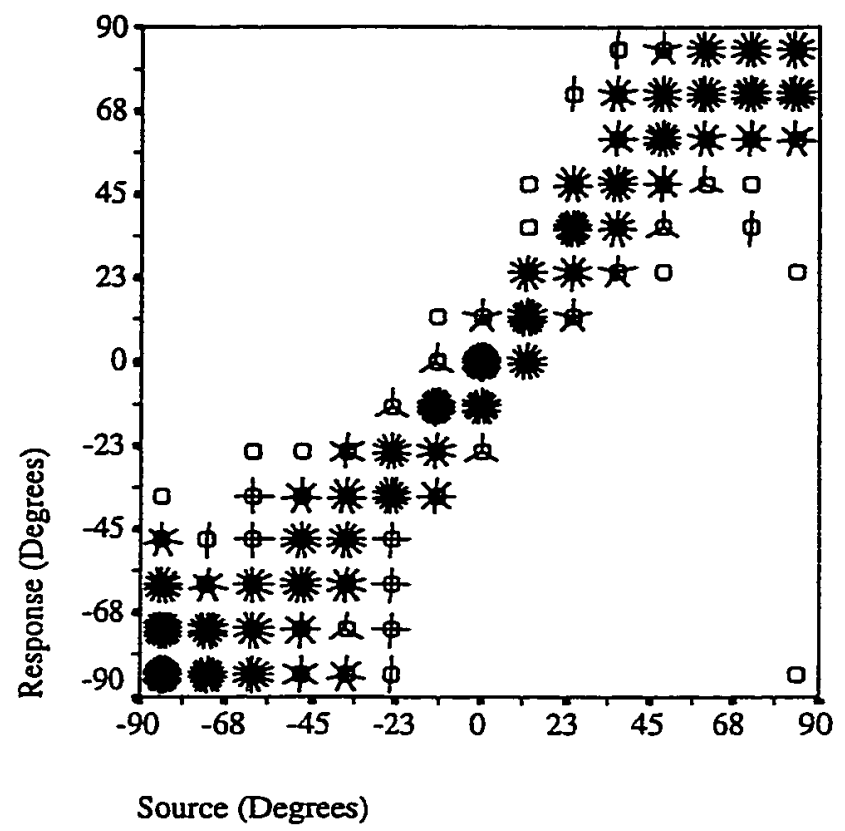

Figure 68: $2.0 \mathrm{kHz}$ to $4.0 \mathrm{kHz}$ band-pass noise with interaural intensity differences unattenuated.

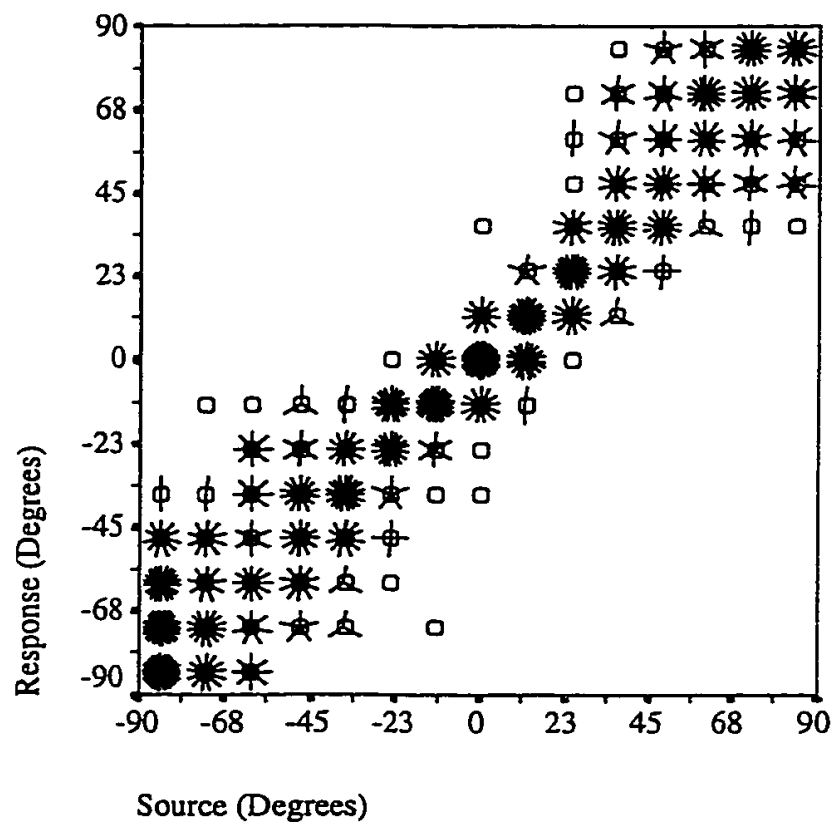

Figure 69: $2.0 \mathrm{kHz}$ to $4.0 \mathrm{kHz}$ band-pass noise with interaural intensity differences attenuated by $50 \%$. 


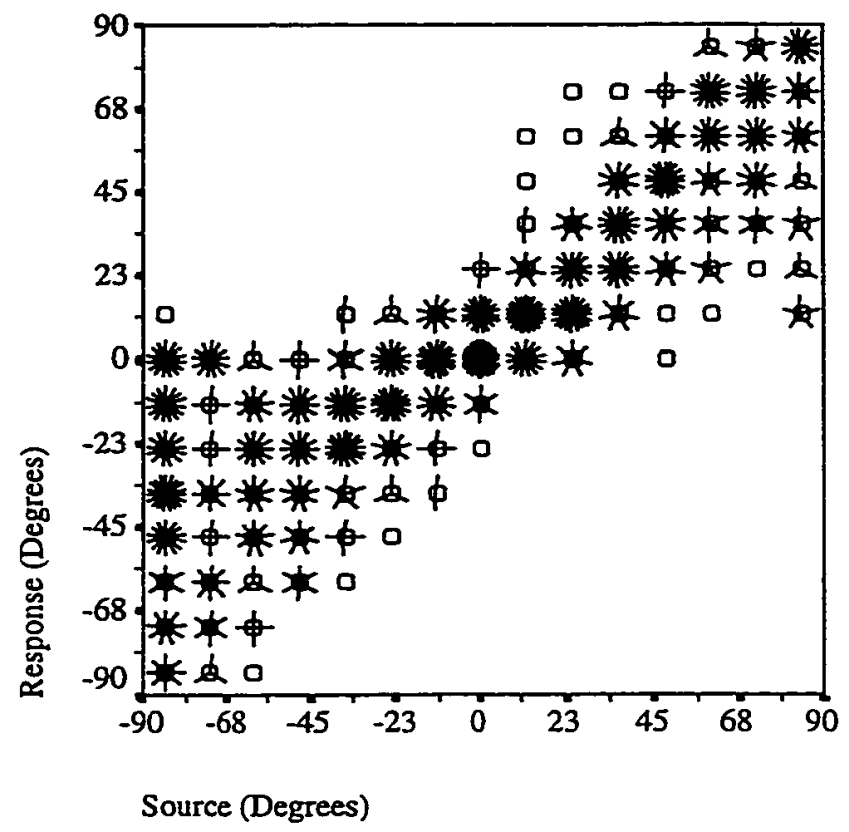

Figure 70: $2.0 \mathrm{kHz}$ to $4.0 \mathrm{kHz}$ band-pass noise with interaural intensity differences attenuated by $100 \%$.

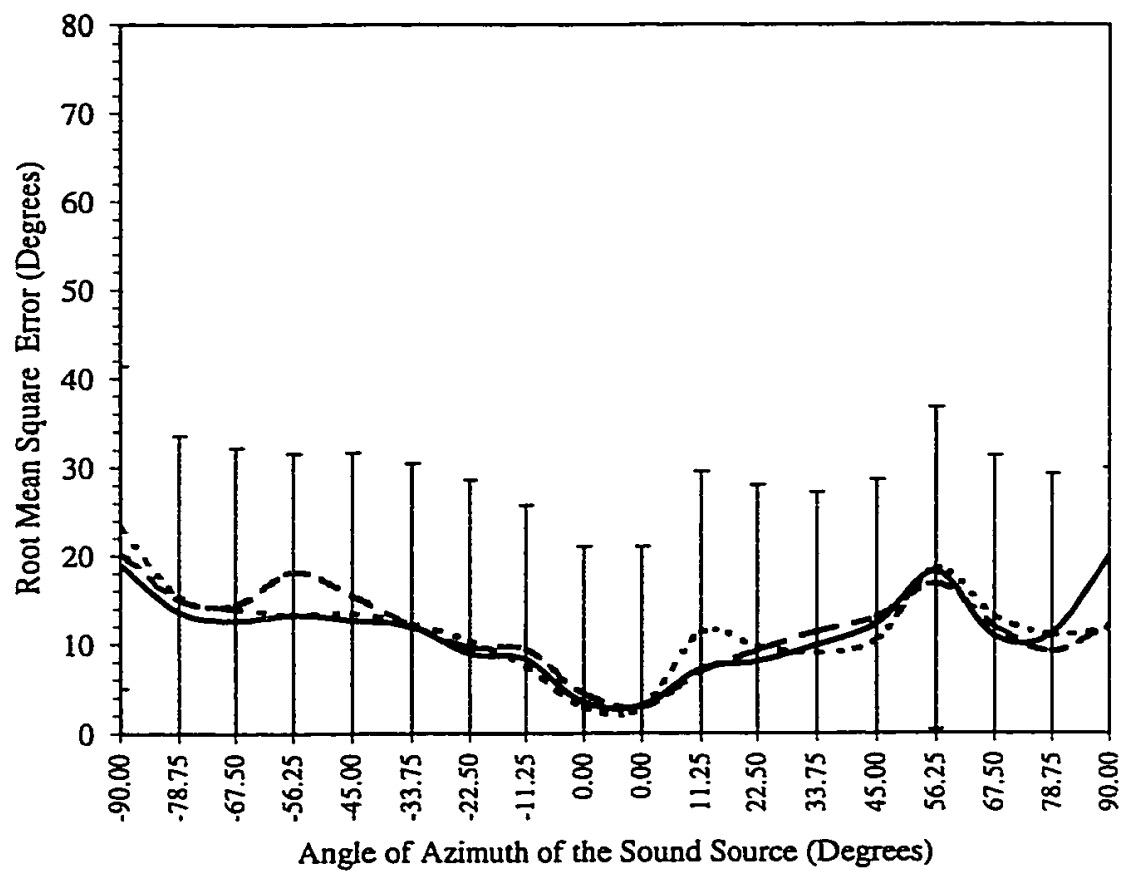

Figure 71: Effect of interaural intensity difference condition and azimuth for the 0.5 kHz third-octave band. 


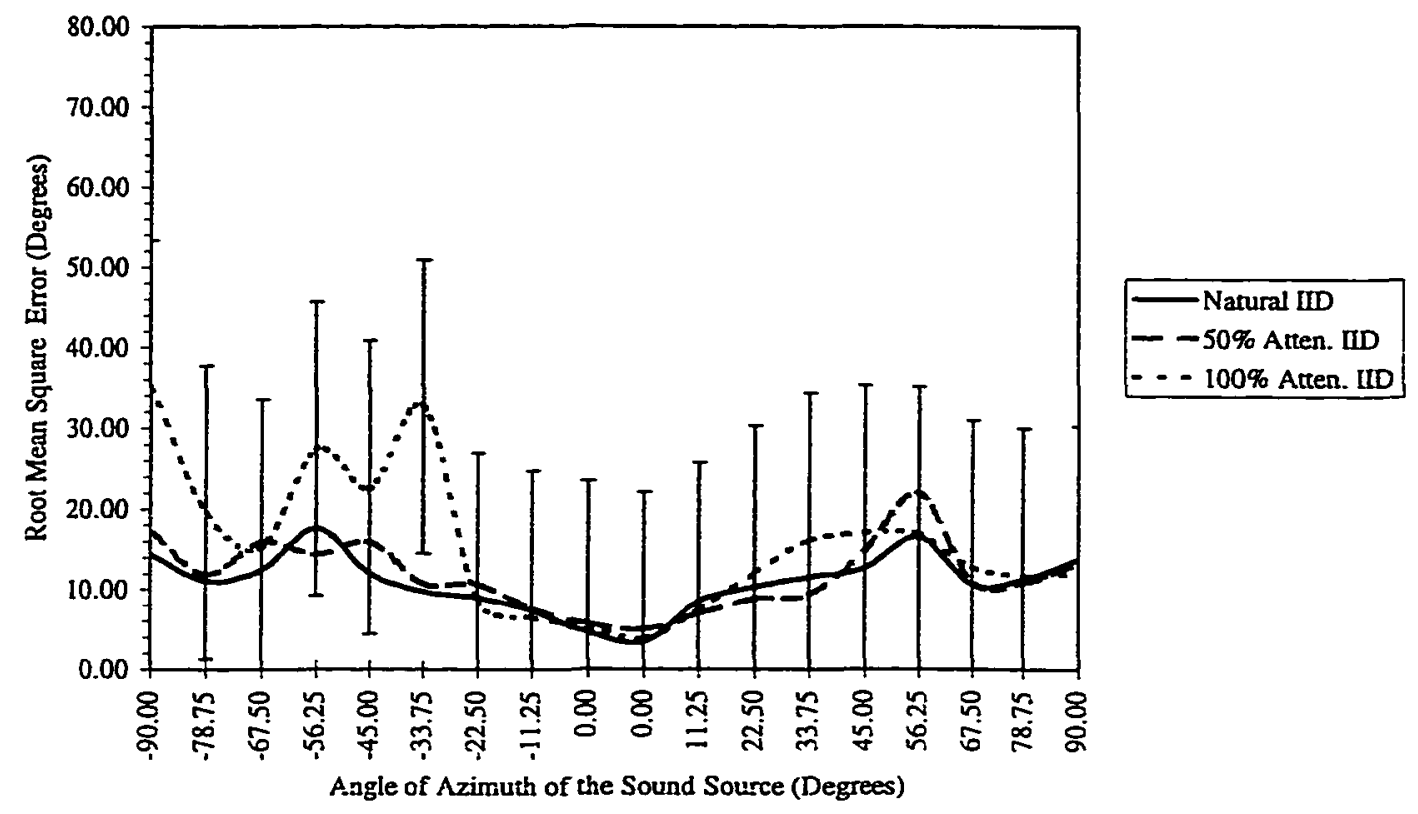

Figure 72: Effect of interaural intensity condition and azimuth for the $1.0 \mathrm{kHz}$ third-octave band.

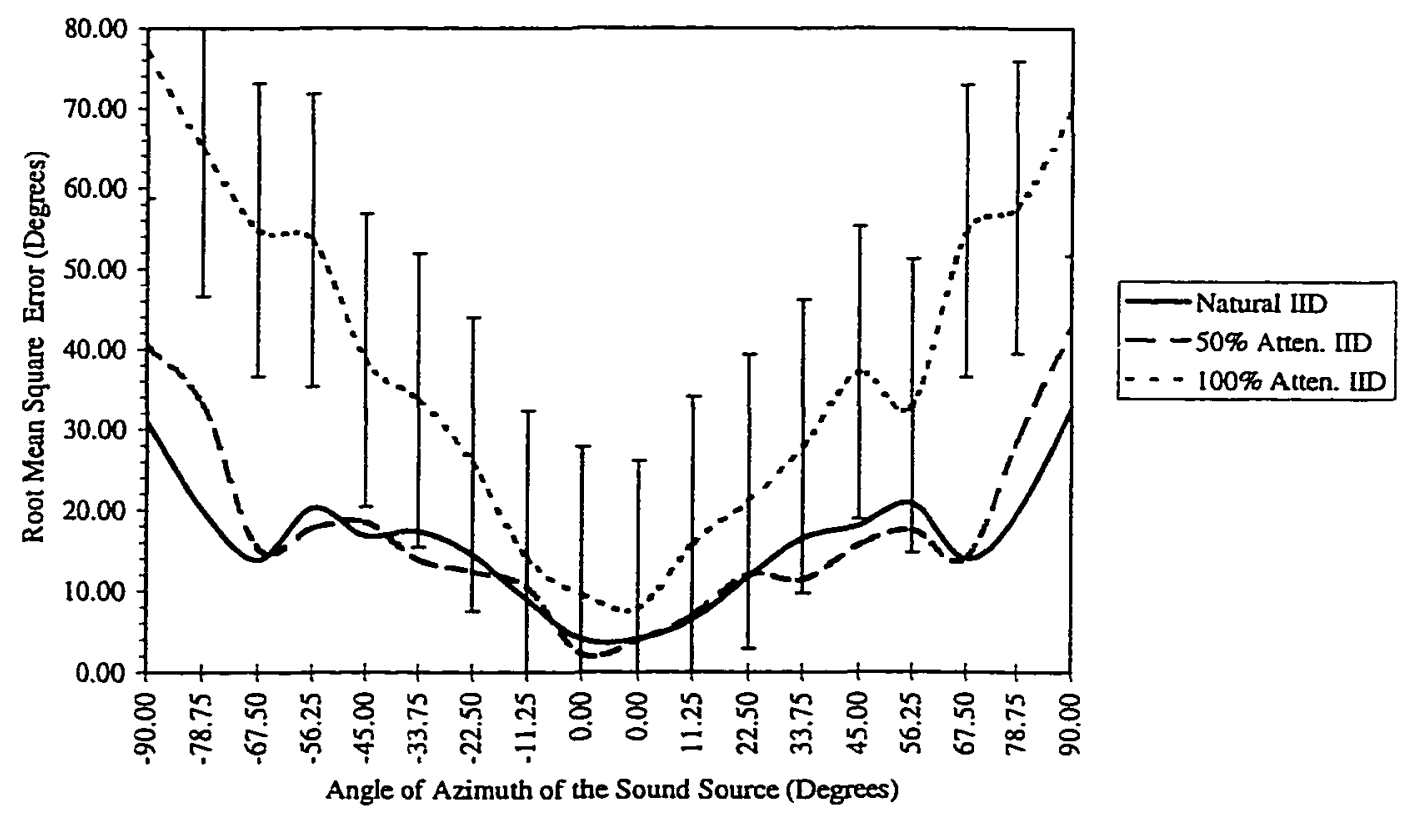

Figure 73: Effect of interaural intensity difference condition and azimuth for the $\mathbf{2 . 0}$ $\mathrm{kHz}$ third-octave band. 


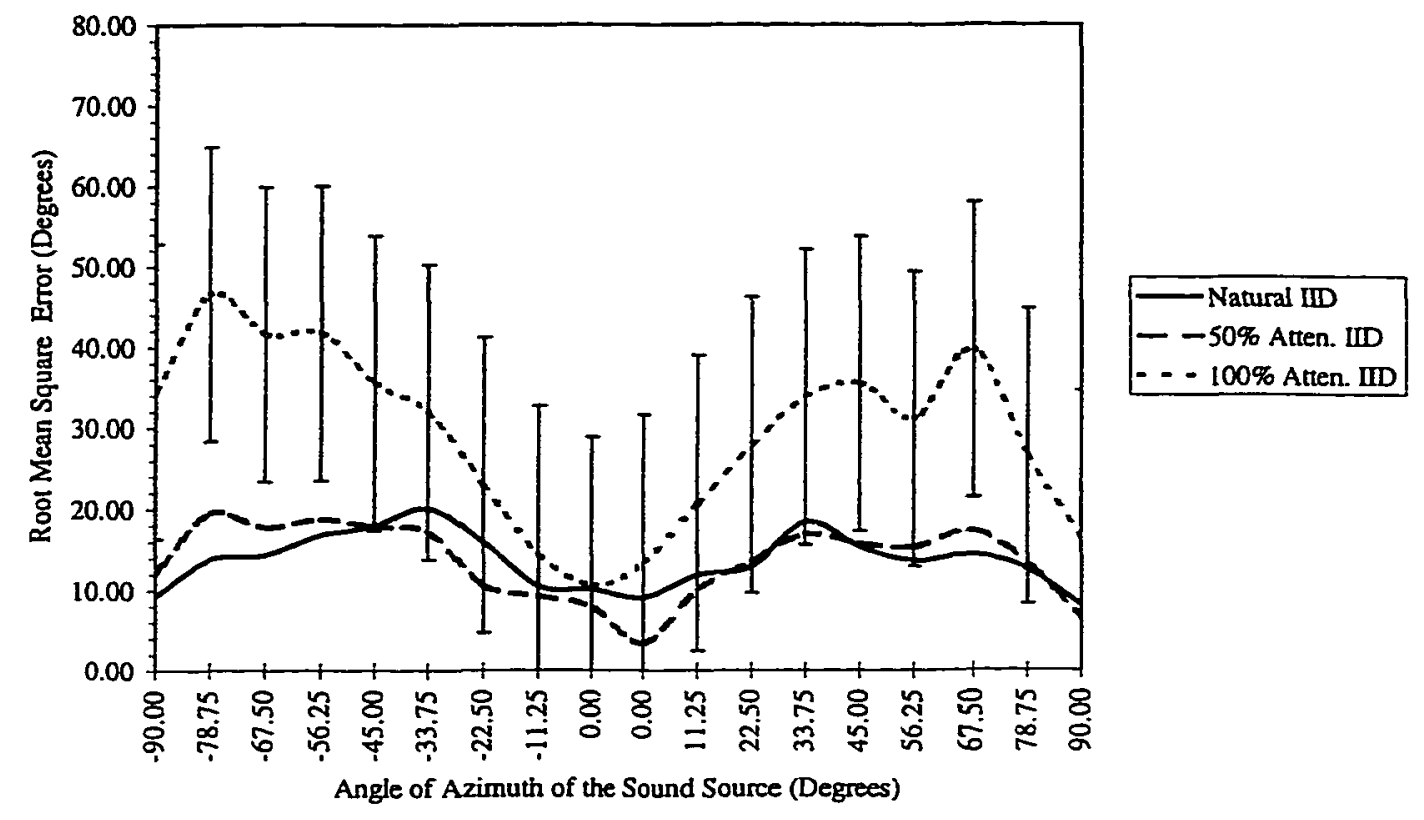

Figure 74: Effect of interaural intensity difference condition and azimuth for the 4.0 kHz third-octave band.

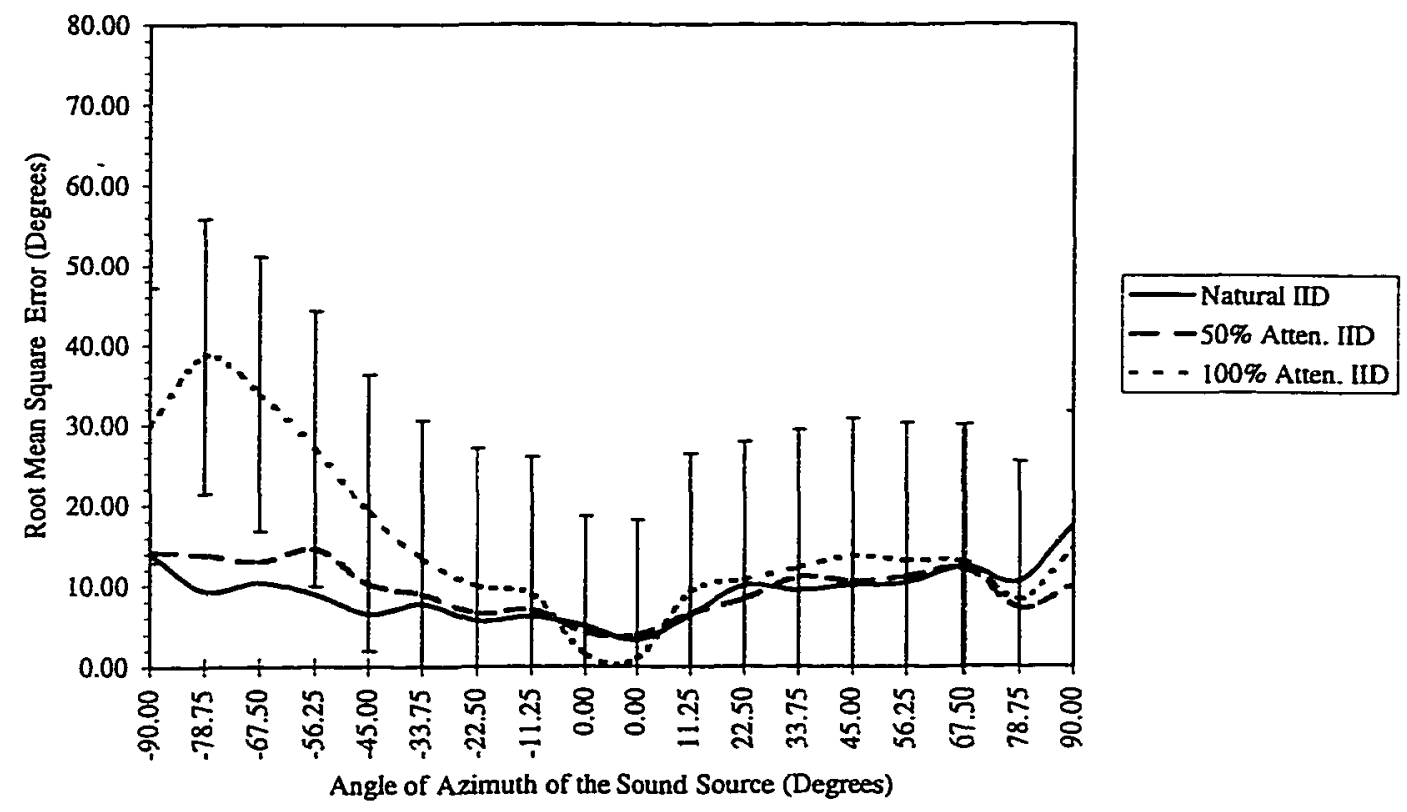

Figure 75: Effect of interaural intensity difference Condition and azimuth for the $0.5 \mathrm{kHz}$ to $4.0 \mathrm{kHz}$ band-pass noise. 


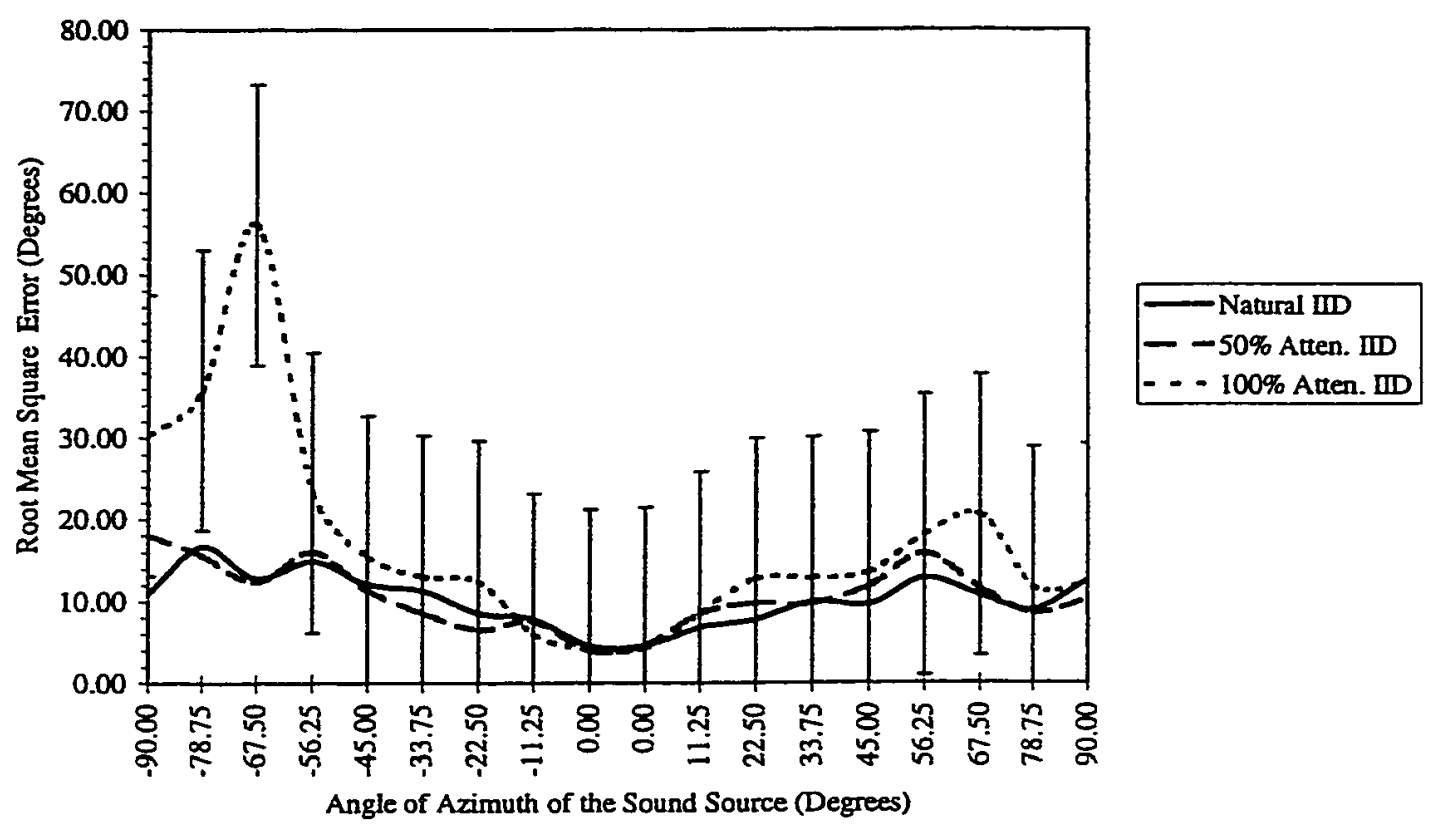

Figure 76: Effect of interaural intensity difference condition and azimuth for the 1.0 $\mathrm{kHz}$ to $4.0 \mathrm{kHz}$ band-pass noise.

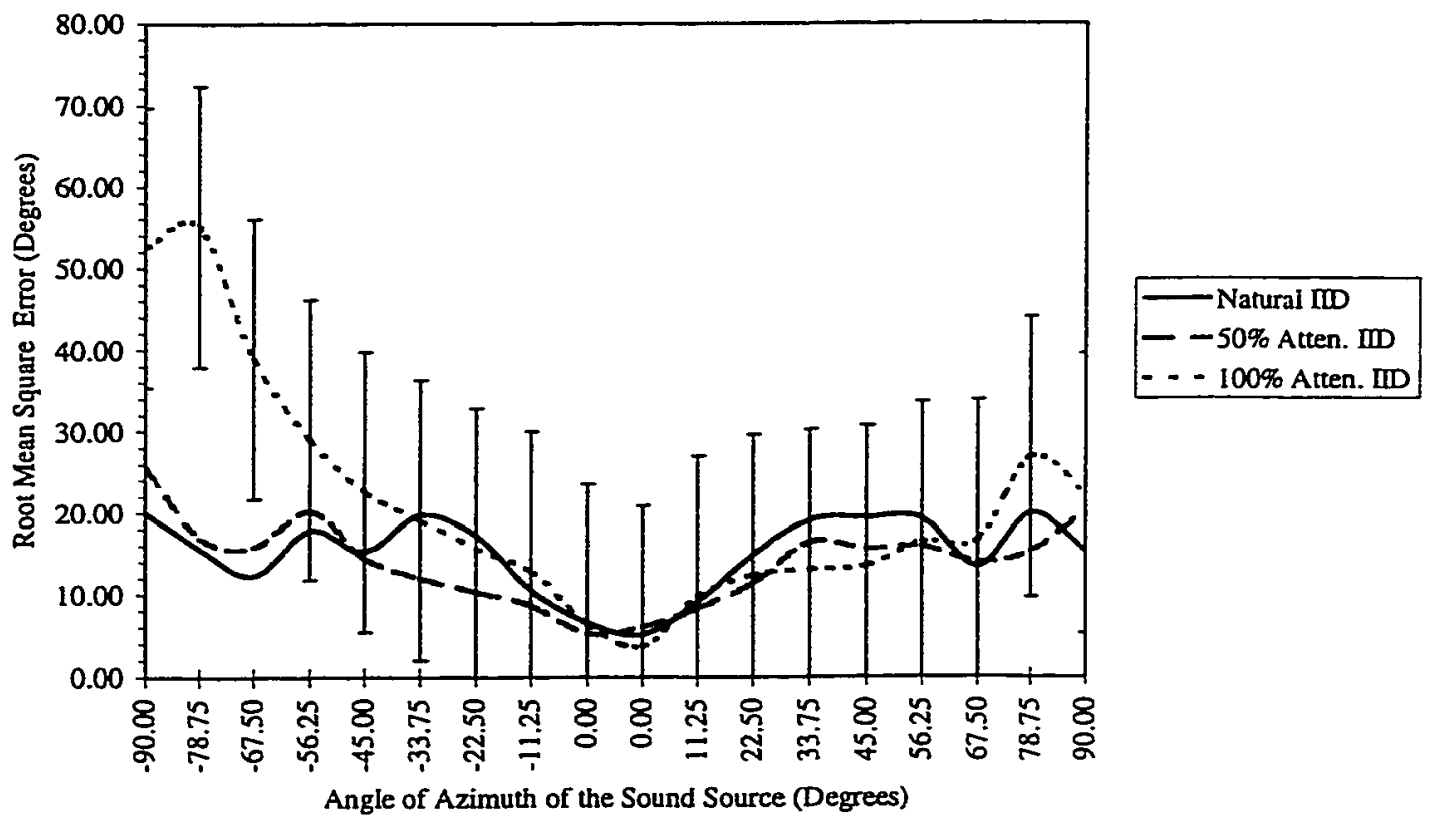

Figure 77: Effect of interaural intensity difference condition and azimuth for the $\mathbf{2 . 0}$ $\mathrm{kHz}$ to $4.0 \mathrm{kHz}$ band-pass noise. 


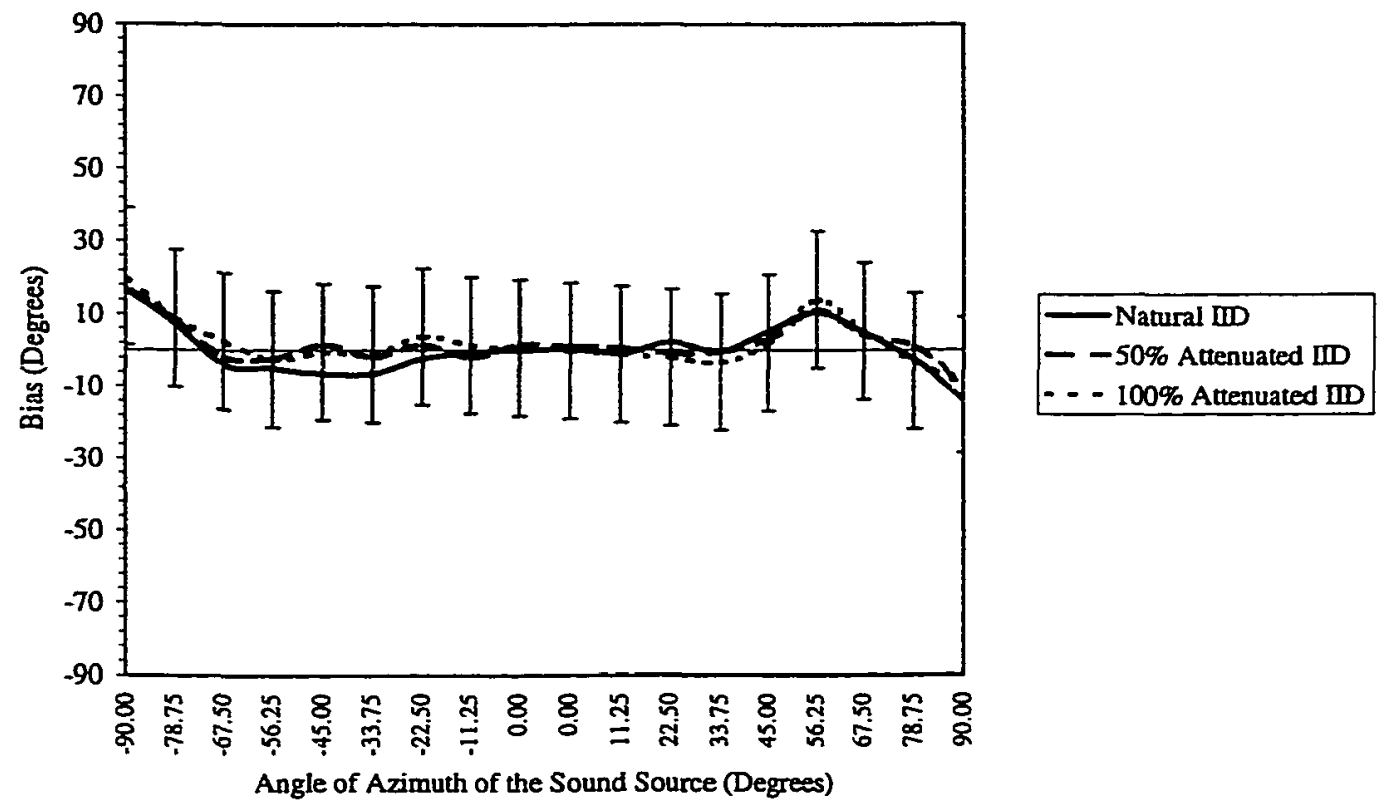

Figure 78: Effect of interaural intensity difference condition, azimuth and signal frequency on bias for the $0.5 \mathrm{kHz}$ third-octave band noise.
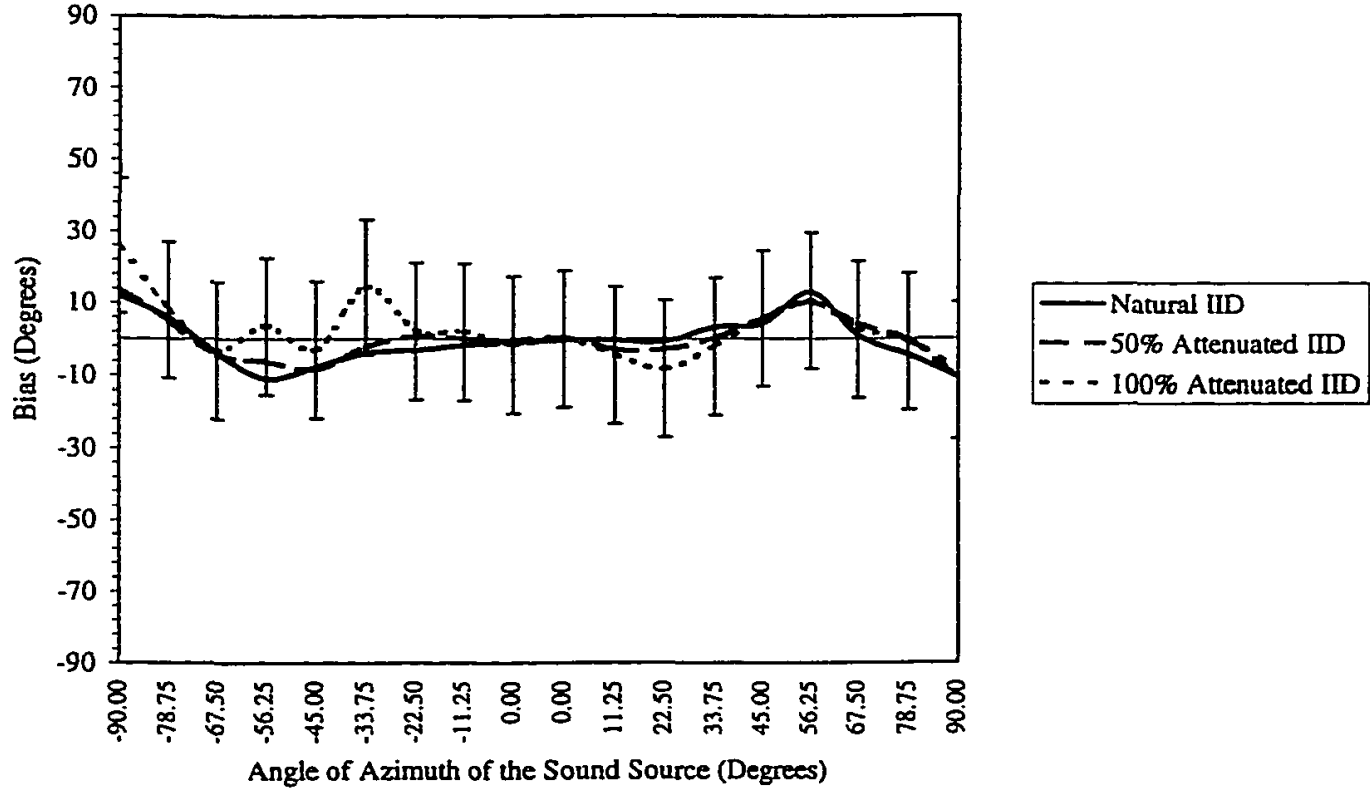

Figure 79: Effect of interaural intensity difference condition, azimuth and signal frequency on bias for the $1.0 \mathrm{kHz}$ third-octave band noise. 

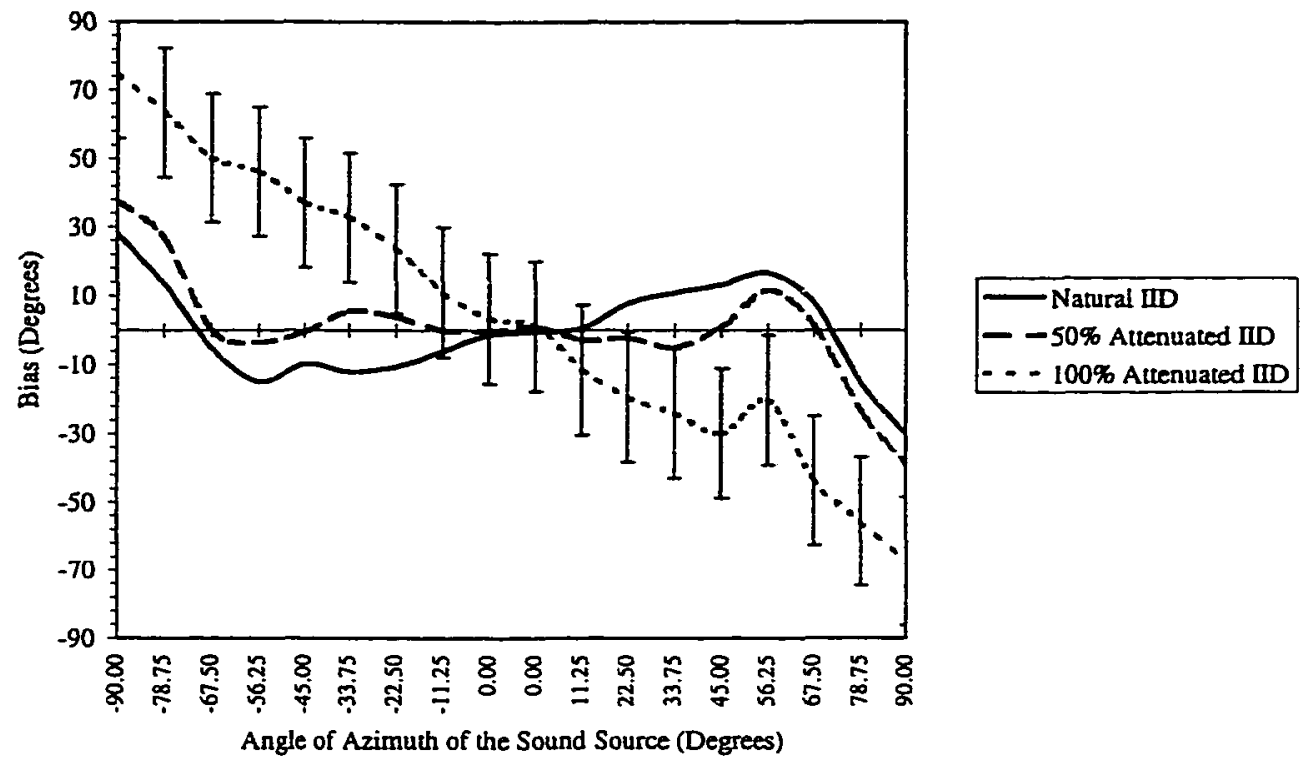

Figure 80: Effect of interaural intensity difference condition, azimuth and signal frequency on bias for the $2.0 \mathrm{kHz}$ third-octave band noise.

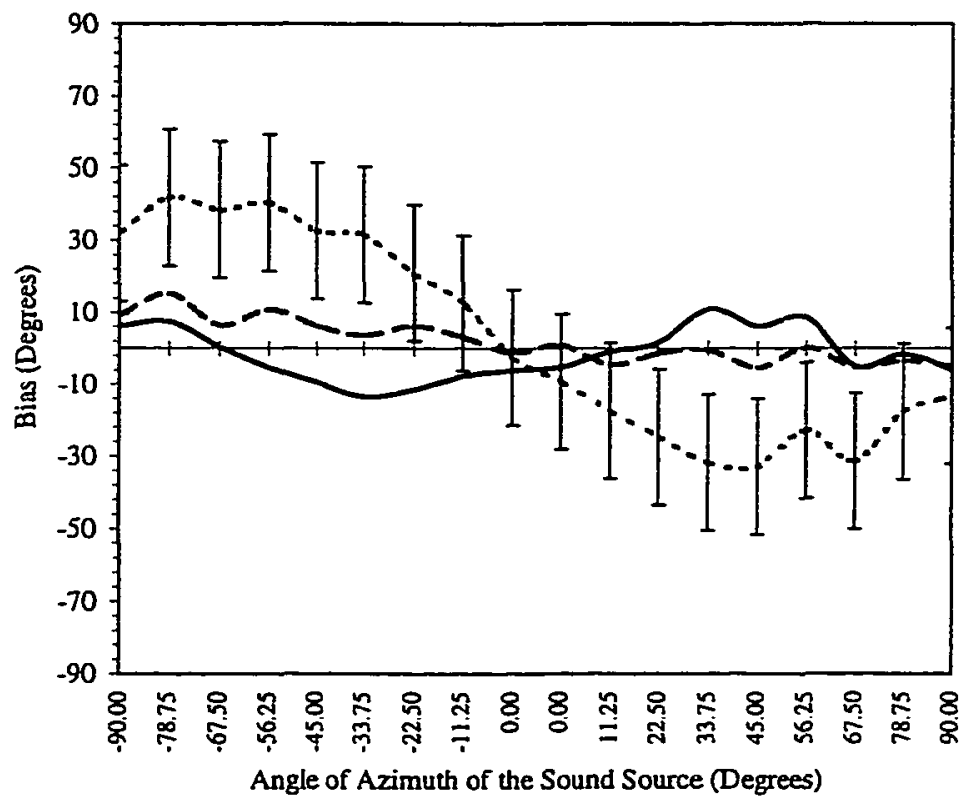

Figure 81: Effect of interaural intensity difference condition, azimuth and signal frequency on bias the $4.0 \mathrm{kHz}$ for third-octave band noise. 


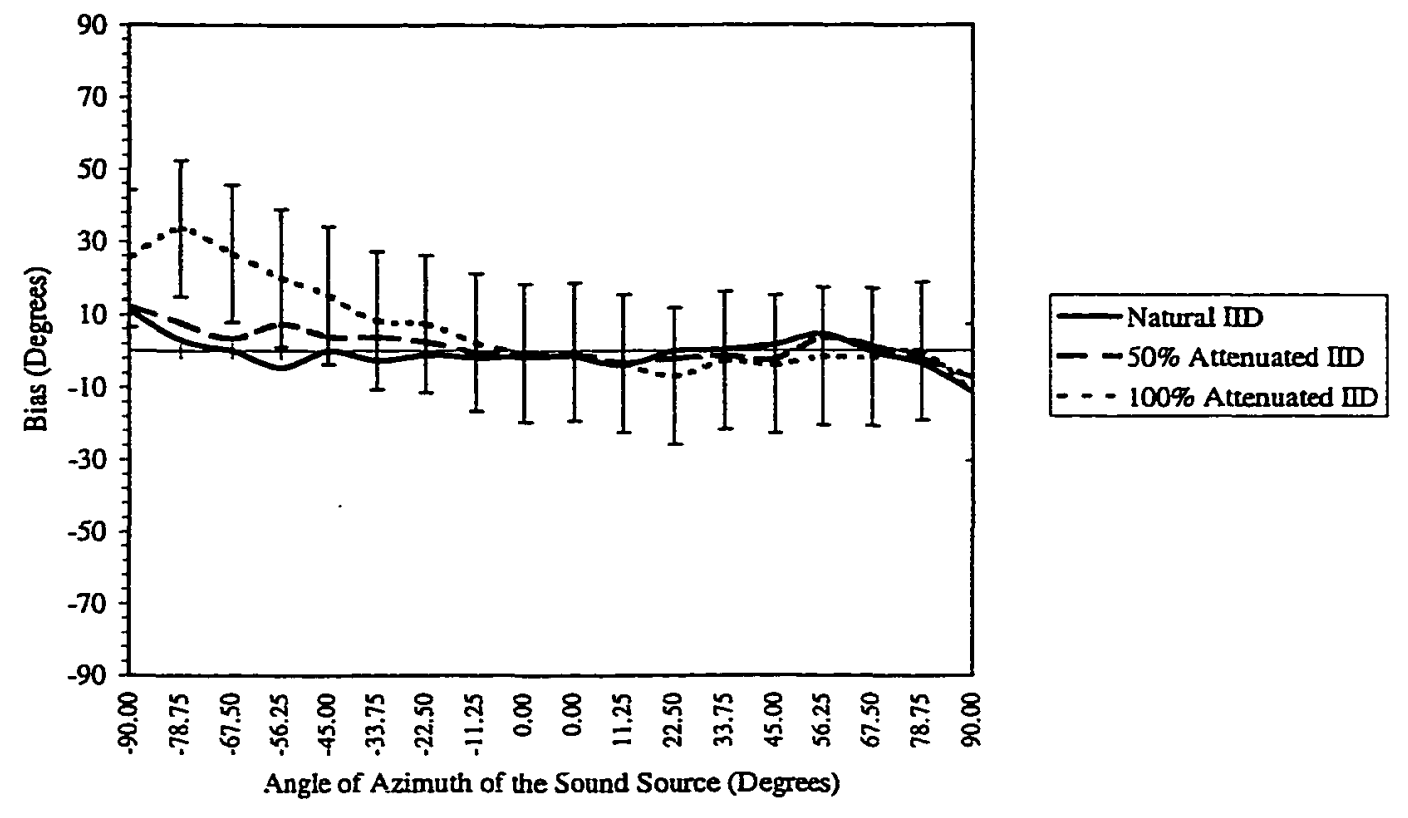

Figure 82: Effect of interaural intensity difference condition, azimuth and signal frequency on bias for the $0.5 \mathrm{kHz}$ to $4.0 \mathrm{kHz}$ band-pass noise.
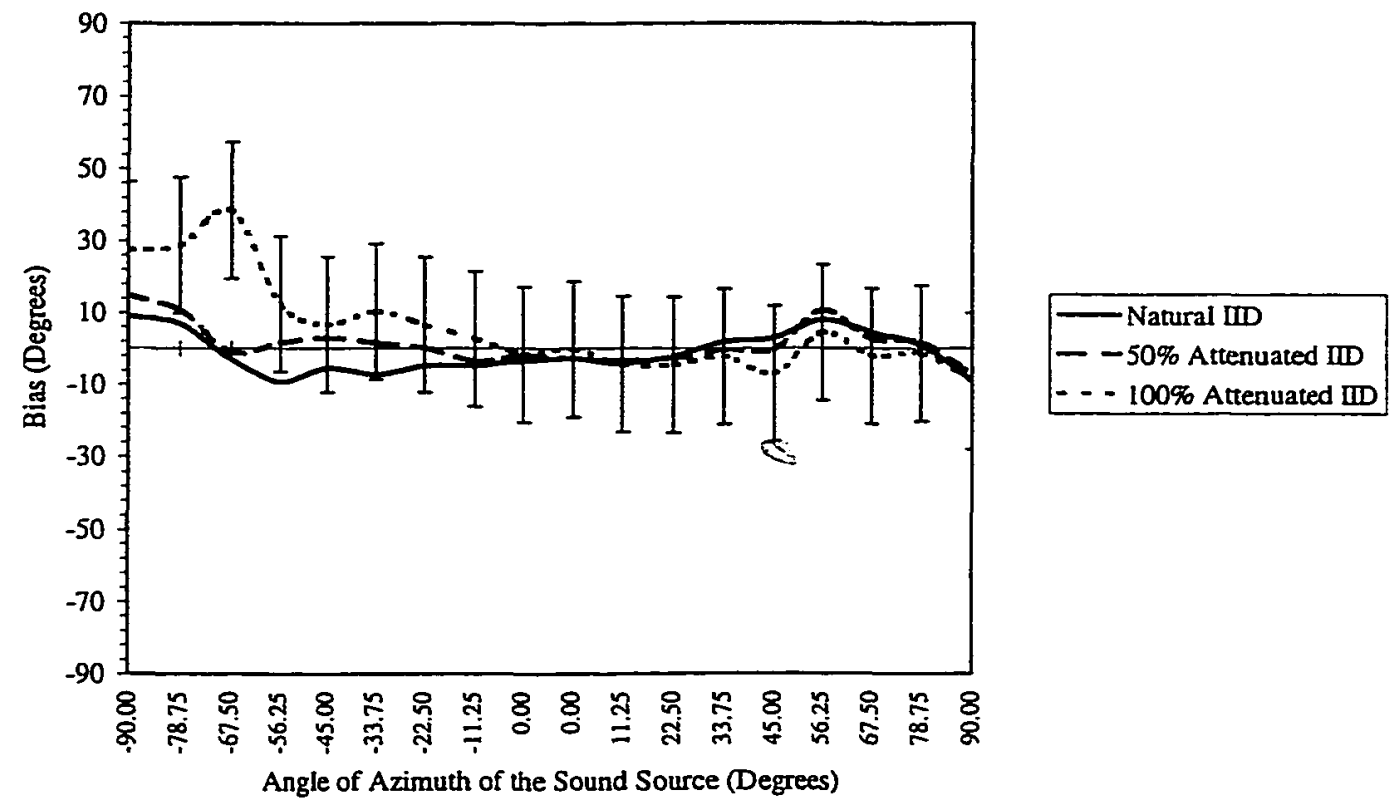

Figure 83: Effect of interaural intensity difference condition, azimuth and signal frequency on bias for the $1.0 \mathrm{kHz}$ to $4.0 \mathrm{kHz}$ band-pass noise. 


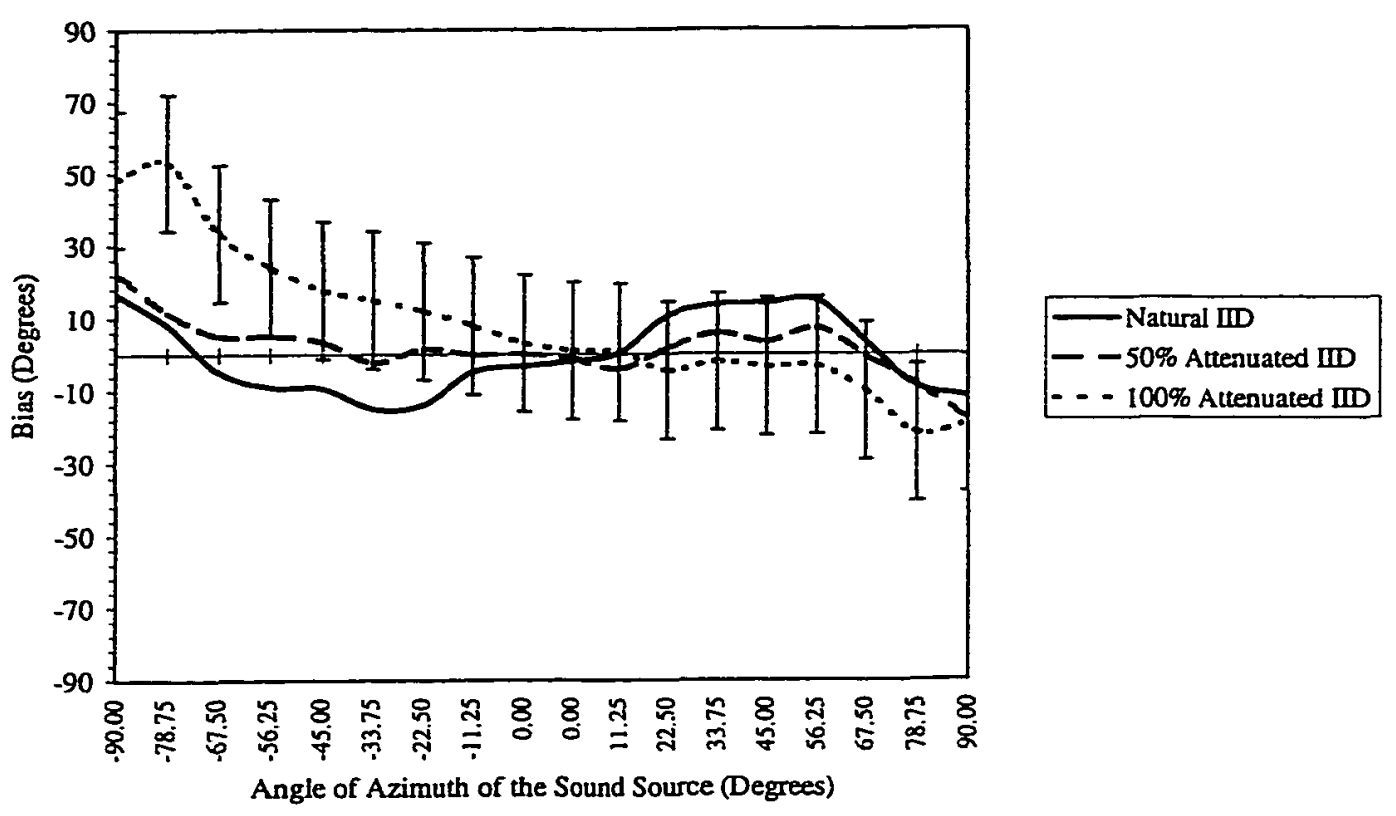

Figure 84: Effect of interaural intensity difference condition, azimuth and signal frequency on bias for the $2.0 \mathrm{kHz}$ to $4.0 \mathrm{kHz}$ band-pass noise.
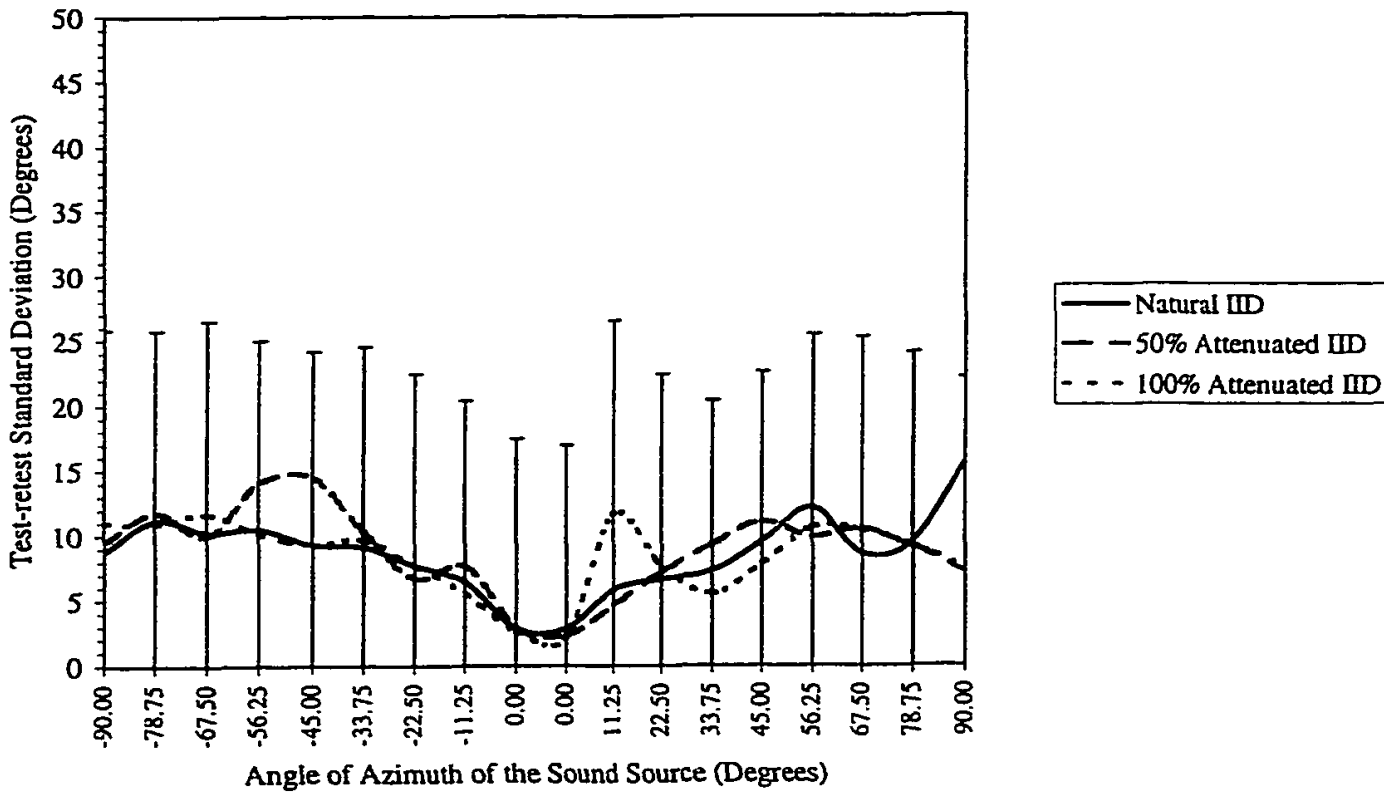

Figure 85: Effect of azimuth and interaural intensity difference condition for the 0.5 $\mathrm{kHz}$ third-octave band of noise. 

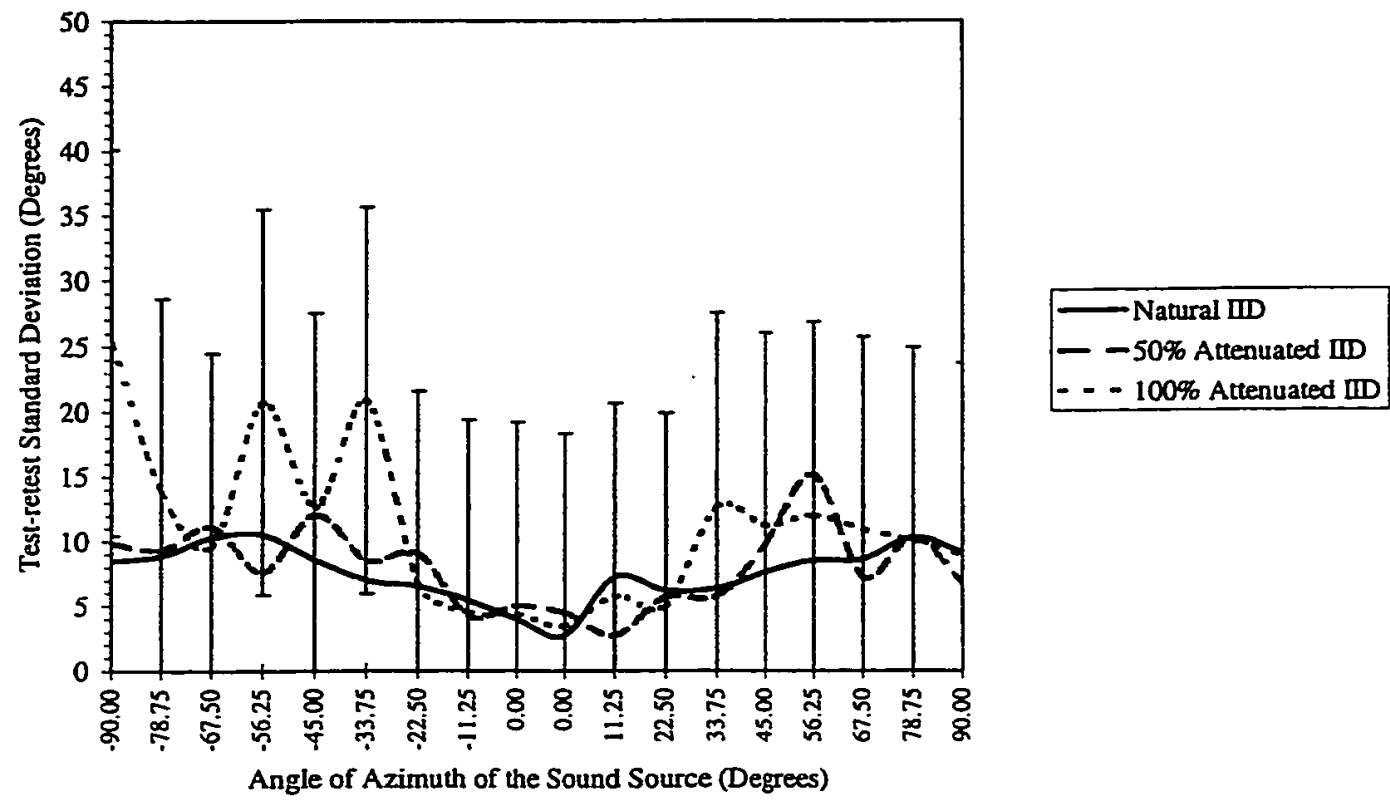

Figure 86: Effect of azimuth and interaural intensity difference condition for the $\mathbf{1 . 0}$ $\mathrm{kHz}$ third-octave band of noise.
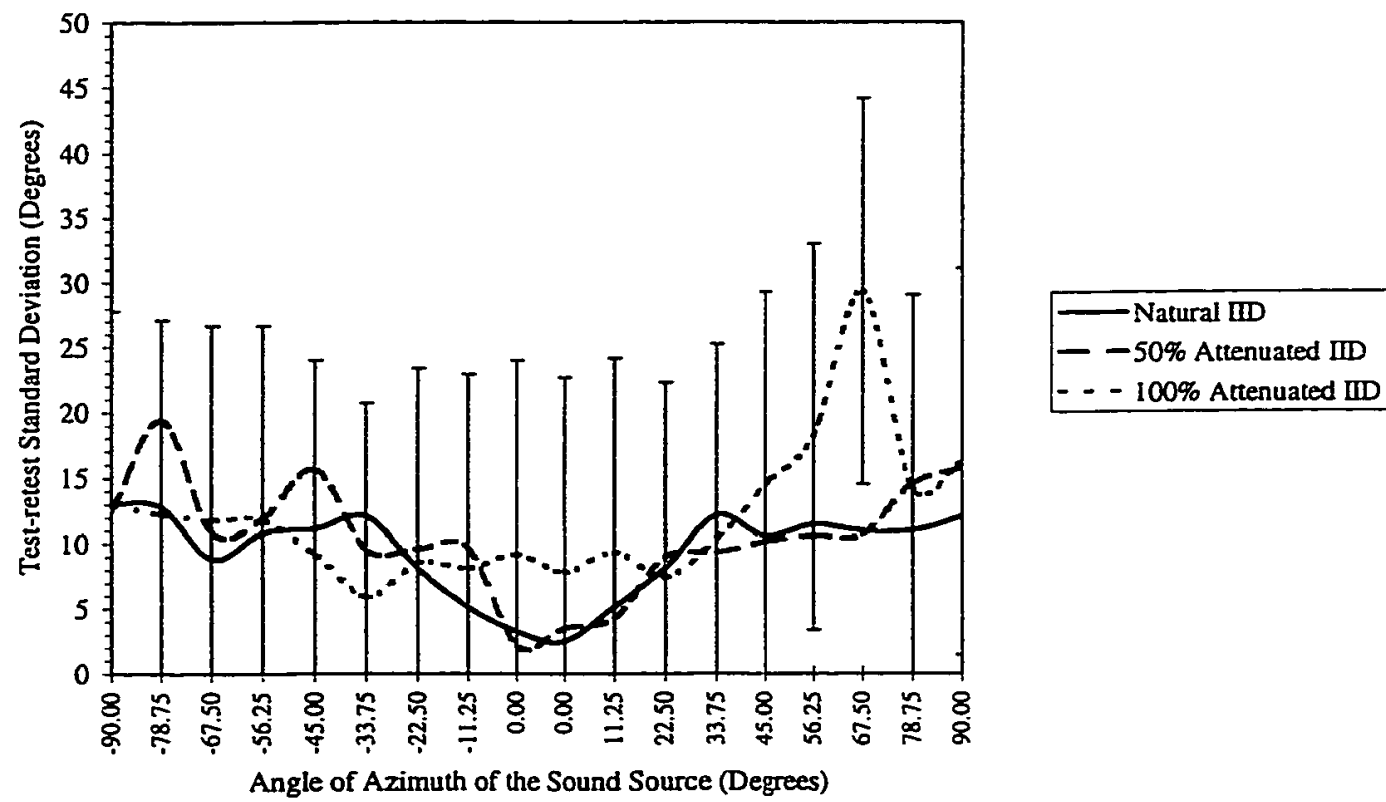

Figure 87: Effect of azimuth and interaural intensity difference condition for the 2.0 $\mathrm{kHz}$ third-octave band of noise. 

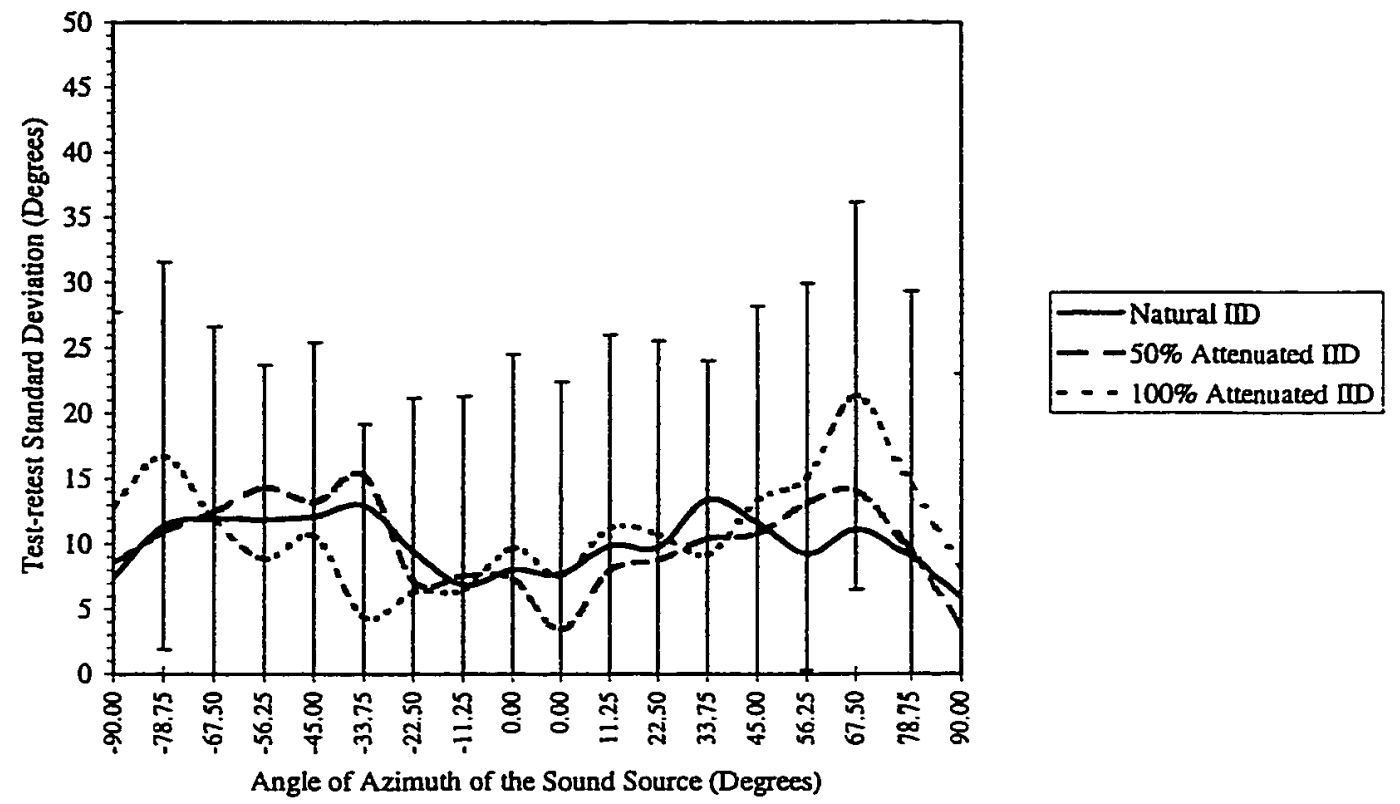

Figure 88: Effect of azimuth and interaural intensity difference condition for the 4.0 kHz third-octave band of noise.
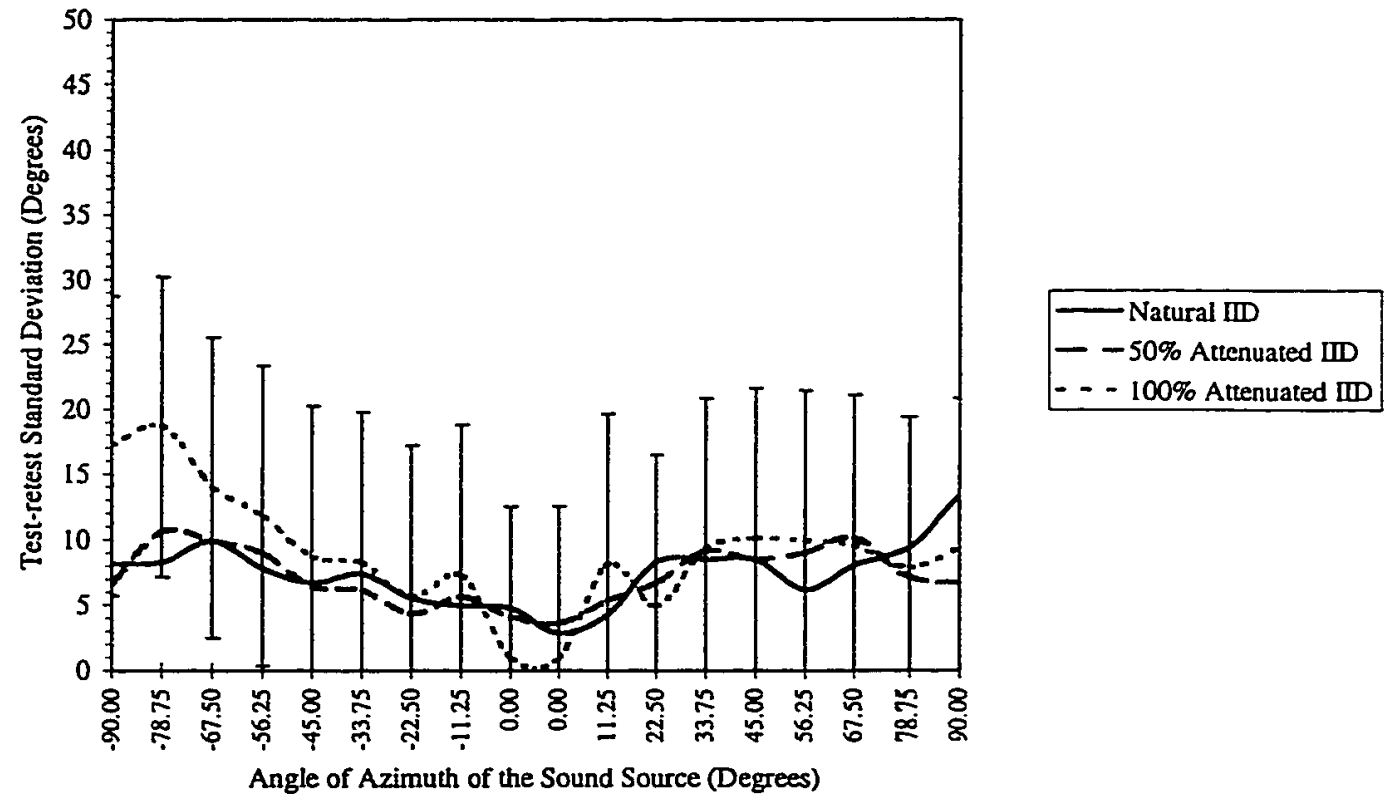

Figure 89: Effect of azimuth and interaural intensity difference condition for the 0.5 $\mathrm{kHz}$ to $4.0 \mathrm{kHz}$ band-pass noise. 

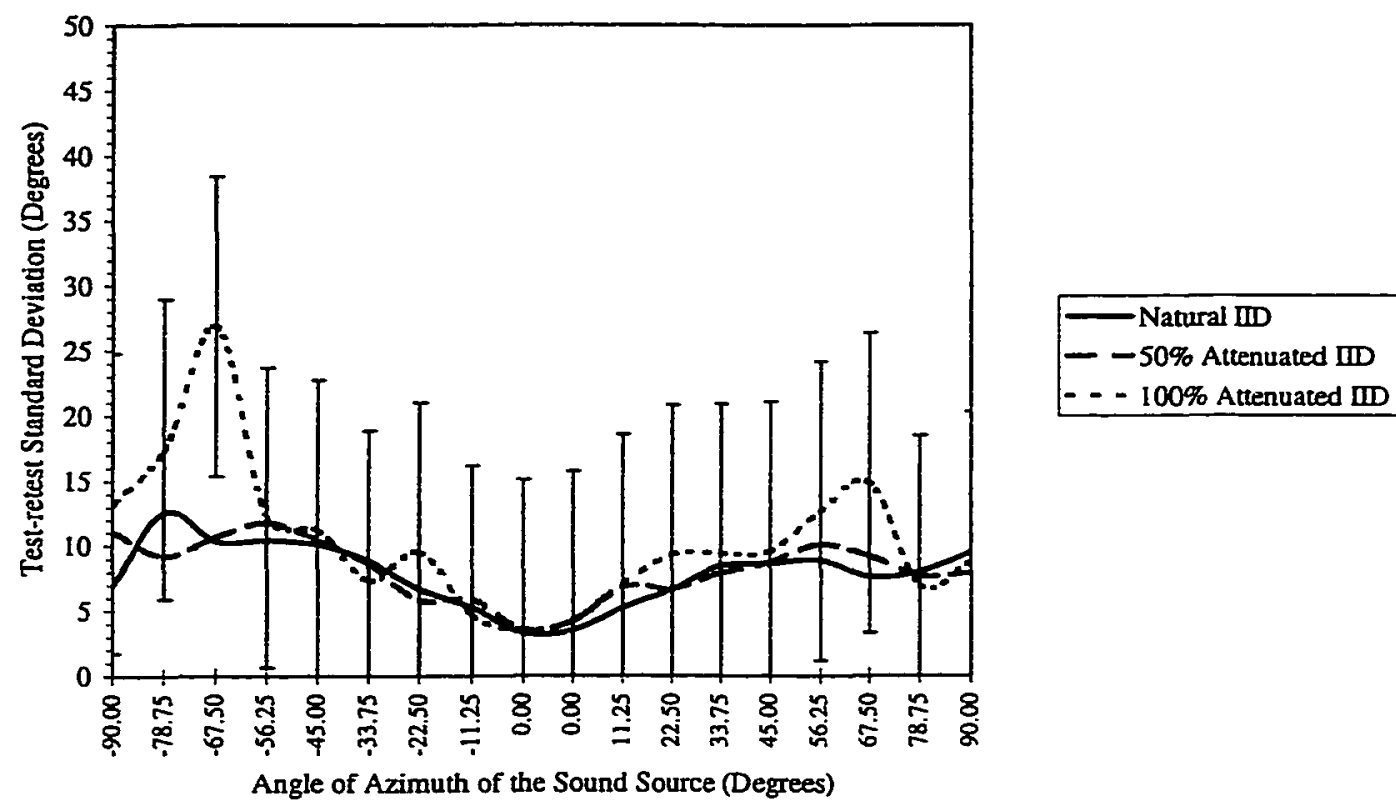

Figure 90: Effect of azimuth and interaural intensity difference condition for the 1.0 to $4.0 \mathrm{kHz}$ band-pass noise.
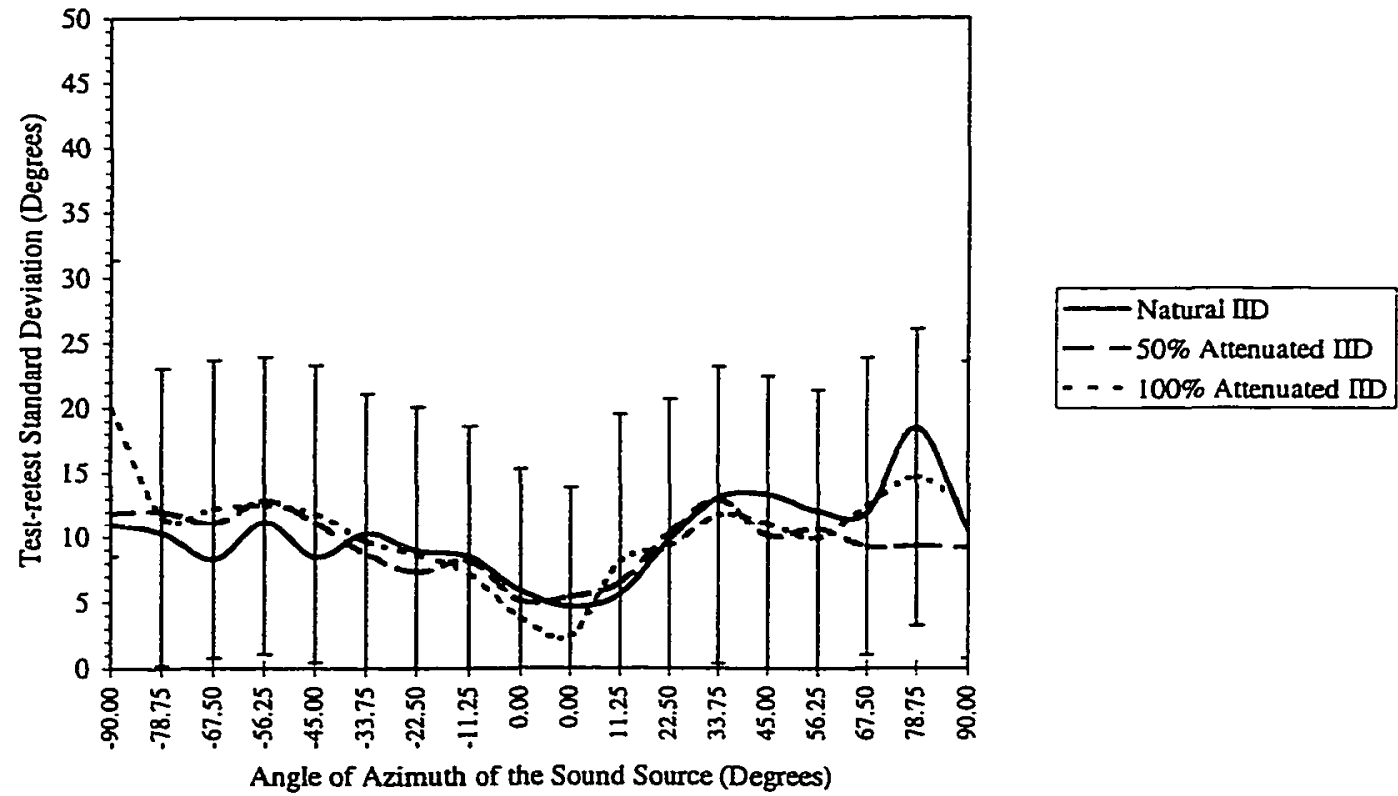

Figure 91: Effect of azimuth and interaural intensity difference condition for the 2.0 to $4.0 \mathrm{kHz}$ band-pass noise. 
Table 12: ANOVA table of the absolute value of the bias using side (left/right) as a factor, for the third-octave bands of noise.

\begin{tabular}{|c|c|c|c|c|c|}
\hline \multicolumn{6}{|c|}{ Tests of Within-Subjects Effects } \\
\hline \multicolumn{6}{|c|}{ Bias - Third-octave Band Noises - Side is a Factor } \\
\hline \multicolumn{6}{|c|}{ Sphericity Assumed } \\
\hline Source & $\begin{array}{l}\text { Type III } \\
\text { Sum of } \\
\text { Squares }\end{array}$ & df & $\begin{array}{l}\text { Mean } \\
\text { Square }\end{array}$ & $\mathbf{F}$ & Sig. \\
\hline ROOM & 5.975 & 1 & 5.975 & 2.452 & .192 \\
\hline Error(ROOM) & 9.748 & 4 & 2.437 & & \\
\hline FREQ & 433.436 & 3 & 144.479 & 35.000 & .000 \\
\hline Error(FREQ) & 49.536 & 12 & 4.128 & & \\
\hline IID & 424.306 & 2 & 212.153 & 40.507 & .000 \\
\hline Error(iiD) & 41.899 & 8 & 5.237 & & \\
\hline SIDE & 21.044 & 1 & 21.044 & 10.906 & .030 \\
\hline Error(SDE) & 7.718 & 4 & 1.930 & & \\
\hline AZIM & 461.616 & 8 & 57.702 & 26.806 & .000 \\
\hline Error(AZIM) & 68.882 & 32 & 2.153 & & \\
\hline ROOM * FREQ & 3.428 & 3 & 1.143 & 1.681 & .224 \\
\hline Error(ROOM*FREQ) & 8.156 & 12 & .680 & & \\
\hline ROOM * II & 237 & 2 & .119 & .067 & .936 \\
\hline Error(ROOM*ID) & 14.275 & 8 & 1.784 & & \\
\hline FREQ * IID & 328.656 & 6 & 54.776 & 63.093 & .000 \\
\hline Error(FREQ*IID) & 20.836 & 24 & .868 & & \\
\hline ROOM * FREQ * IID & 5.458 & 6 & .910 & 1.652 & .176 \\
\hline Error(ROOM*FREQ*ID) & 13.218 & 24 & .551 & & \\
\hline ROOM * SIDE & 3.205 & 1 & 3.205 & 3.618 & .130 \\
\hline Error(ROOM*SIDE) & 3.543 & 4 & .886 & & \\
\hline$\overline{\text { FREQ } * \text { SIDE }}$ & 1.213 & 3 & .404 & .446 & .725 \\
\hline Error(FREQ*SDE) & 10.885 & 12 & .907 & & \\
\hline ROOM * FREQ * SIDE & 3.483 & 3 & 1.161 & .961 & .442 \\
\hline Error(ROOM*FREQ*SIDE) & 14.492 & 12 & 1.208 & & \\
\hline IID * SDE & 18.383 & 2 & 9.191 & 3.108 & .100 \\
\hline Error(IID*SIDE) & 23.662 & 8 & 2.958 & & \\
\hline $\mathrm{ROOM} * \mathrm{mD} * \mathrm{SIDE}$ & .245 & 2 & .123 & .125 & .884 \\
\hline Error(ROOM*IID*SIDE) & 7.839 & 8 & .980 & & \\
\hline FREQ * ID * SIDE & 5.646 & 6 & .941 & .844 & .548 \\
\hline Error(FREQ*II $*$ SIDE) & 26.743 & 24 & 1.114 & & \\
\hline $\mathrm{ROOM}^{*} \mathrm{FREQ}^{*} \mathrm{ID} * \mathrm{SIDE}$ & 4.339 & 6 & .723 & 1.299 & 296 \\
\hline Error(ROOM*FREQ*IID*SIDE) & 13.365 & 24 & .557 & & \\
\hline ROOM * AZIM & 9.873 & 8 & 1.234 & 2.387 & .038 \\
\hline Error(ROOM*AZTM) & 16.546 & 32 & .517 & & \\
\hline FREQ * AZIM & 388.077 & 24 & 16.170 & 14.411 & .000 \\
\hline Error(FREQ*AZIM) & 107.719 & 96 & 1.122 & & \\
\hline
\end{tabular}




\begin{tabular}{|c|c|c|c|c|c|}
\hline ROOM * FREQ * AZIM & 20.517 & 24 & .855 & 1.881 & .017 \\
\hline Error(ROOM*FREQ*AZMM) & 43.631 & 96 & .454 & & \\
\hline IID * AZM & 114.392 & 16 & 7.149 & 12.251 & .000 \\
\hline Error(III*AZIM) & 37.348 & 64 & .584 & & \\
\hline ROOM * IID * AZIM & 11.829 & 16 & .739 & 2.299 & .010 \\
\hline Error(ROOM*ID*AZM) & 20.577 & 64 & .322 & & \\
\hline FREQ * IID * AZIM & 132.118 & 48 & 2.752 & 5.397 & .000 \\
\hline Error(FREQ*IID*AZIM) & 97.911 & 192 & .510 & & \\
\hline ROOM * FREQ * II * AZIM & 39.948 & 48 & .832 & 2.979 & .000 \\
\hline Error(ROOM*FREQ*II $*$ AZIM) & 53.645 & 192 & .279 & & \\
\hline SIDE * AZIM & 18.769 & 8 & 2.346 & 1.459 & .211 \\
\hline Error(SIDE*AZIM) & 51.450 & 32 & 1.608 & & \\
\hline ROOM * SIDE * AZIM & 4.788 & 8 & .599 & 2.125 & .062 \\
\hline Error(ROOM*SIDE*AZMM) & 9.015 & 32 & .282 & & \\
\hline FREQ * SIDE * AZIM & 20.523 & 24 & .855 & 1.570 & .065 \\
\hline Error(FREQ*SIDE*AZIM) & 52.284 & 96 & .545 & & \\
\hline ROOM * FREQ * SIDE * AZIM & 12.158 & 24 & .507 & 1.231 & .237 \\
\hline Error(ROOM*FREQ*SIDE*AZIM) & 39.521 & 96 & .412 & & \\
\hline II * SIDE * AZIM & 24.068 & 16 & 1.504 & 3.119 & .001 \\
\hline Error(IID*SIDE*AZIM) & 30.865 & 64 & .482 & & \\
\hline ROOM * ID * SIDE * AZTM & 4.024 & 16 & .251 & .737 & .747 \\
\hline Error(ROOM*IID*SIDE*AZIM) & 21.851 & 64 & .341 & & \\
\hline FREQ * ID * SIDE * AZIM & 25.330 & 48 & .528 & 1.540 & .022 \\
\hline Error(FREQ*WD*SIDE*AZIM) & 65.797 & 192 & .343 & & \\
\hline $\begin{array}{l}\text { ROOM * FREQ * IID * SDE * } \\
\text { AZIM }\end{array}$ & 14.462 & 48 & .301 & 1.100 & .320 \\
\hline $\begin{array}{l}\text { Error(ROOM*FREQ*IID*SIDE*A } \\
\text { ZIM) }\end{array}$ & 52.570 & 192 & .274 & & \\
\hline
\end{tabular}


Table 13: ANOVA table of the absolute value of the bias using side (left/right) as a factor, for the band-pass noises.

\begin{tabular}{|c|c|c|c|c|c|}
\hline \multicolumn{6}{|c|}{ Tests of Within-Subjects Effects } \\
\hline \multirow{2}{*}{\multicolumn{6}{|c|}{$\frac{\text { Bias - Band-pass Noises - Side is a Factor }}{\text { Sphericity Assumed }}$}} \\
\hline & & & & & \\
\hline Source & \begin{tabular}{|l|} 
Type III \\
Sum of \\
Squares
\end{tabular} & $\mathrm{df}$ & $\begin{array}{l}\text { Mean } \\
\text { Square }\end{array}$ & F & Sig. \\
\hline ROOM & 2.358 & 1 & 2.358 & 1.907 & .239 \\
\hline Error(ROOM) & 4.945 & 4 & 1.236 & & \\
\hline FREQ & 57.031 & 2 & 28.516 & 7.871 & .013 \\
\hline Error(FREQ) & 28.984 & 8 & 3.623 & & \\
\hline IID & 124.689 & 2 & 62.344 & 19.131 & .001 \\
\hline Error(IID) & 26.071 & 8 & 3.259 & & \\
\hline SDE & 55.778 & 1 & 55.778 & 9.694 & .036 \\
\hline Error(SIDE) & 23.016 & 4 & 5.754 & & \\
\hline AZIM & \begin{tabular}{|l|}
232.789 \\
\end{tabular} & 8 & 29.099 & 23.266 & .000 \\
\hline Error(AZTM) & 40.023 & 32 & 1.251 & & \\
\hline ROOM * FREQ & 2.448 & 2 & 1.224 & 3.863 & .067 \\
\hline Error(ROOM*FREQ) & 2.535 & 8 & .317 & & \\
\hline ROOM * IID & 1.033 & 2 & .517 & .731 & .511 \\
\hline Error(ROOM*ID) & 5.651 & 8 & .706 & & \\
\hline FREQ * IID & 1.966 & 4 & .492 & .204 & .933 \\
\hline Error(FREQ*ID) & 38.633 & 16 & 2.415 & & \\
\hline ROOM * FREQ * IID & 5.260 & 4 & 1.315 & 1.825 & .173 \\
\hline Error(ROOM*FREQ*ID) & 11.528 & 16 & .720 & & \\
\hline ROOM * SIDE & .146 & 1 & .146 & .174 & .698 \\
\hline Error(ROOM*SIDE) & 3.374 & 4 & .844 & & \\
\hline FREQ * SIDE & 1.963 & 2 & .982 & .929 & .434 \\
\hline Error(FREQ*SIDE) & 8.453 & 8 & 1.057 & & \\
\hline ROOM *FREQ * SDE & 3.020 & 2 & 1.510 & 2.442 & .149 \\
\hline Error(ROOM*FREQ*SIDE) & 4.946 & 8 & .618 & & \\
\hline III * SIDE & 77.378 & 2 & 38.689 & 8.918 & .009 \\
\hline Error(ID*SIDE) & 34.707 & 8 & 4.338 & & \\
\hline ROOM $*$ III * SIDE & 2.699 & 2 & 1.349 & 2.334 & .159 \\
\hline Error(ROOM*ID*SIDE) & 4.625 & 8 & .578 & & \\
\hline FREQ * II * SIDE & 5.495 & 4 & 1.374 & .777 & .556 \\
\hline Error(FREQ*ID*SIDE) & 28.279 & 16 & 1.767 & & \\
\hline ROOM $*$ FREQ $*$ IID * SIDE & .722 & 4 & .181 & .373 & .824 \\
\hline Error(ROOM*FREQ*IID*SIDE) & 7.738 & 16 & .484 & & \\
\hline ROOM * AZIM & 1.998 & 8 & .250 & .771 & .631 \\
\hline Error(ROOM*AZIM) & 10.371 & 32 & .324 & & \\
\hline$\overline{\text { FREQ * AZIM }}$ & 29.027 & 16 & 1.814 & 2.479 & .005 \\
\hline Error(FREQ*AZIM) & 46.841 & 64 & .732 & & \\
\hline
\end{tabular}




\begin{tabular}{|c|c|c|c|c|c|}
\hline ROOM * FREQ * AZIM & 7.608 & 16 & .476 & 1.595 & .096 \\
\hline Emor(ROOM*FREQ*AZIM) & 19.086 & 64 & 298 & & \\
\hline IID * AZIM & 119.529 & 16 & 7.471 & 11.071 & .000 \\
\hline Error(IID*AZTM) & 43.187 & 64 & .675 & & \\
\hline ROOM * IID * AZIM & 10.807 & 16 & .675 & 3.174 & .001 \\
\hline Error(ROOM*IID*AZIM) & 13.619 & 64 & .213 & & \\
\hline FREQ * IID * AZIM & 42.200 & 32 & 1.319 & 3.190 & .000 \\
\hline Error(FREQ*ID $*$ AZMM) & 52.919 & 128 & .413 & & \\
\hline ROOM * FREQ * II * AZIM & 12.689 & 32 & .397 & 1.790 & .012 \\
\hline Error(ROOM*FREQ*II $*$ AZIM) & 28.353 & 128 & .222 & & \\
\hline SIDE* AZIM & 63.876 & 8 & 7.985 & 3.633 & .004 \\
\hline Error(SIDE*AZIM) & 70.330 & 32 & 2.198 & & \\
\hline ROOM * SIDE* AZM & 4.951 & 8 & .619 & 2.164 & .058 \\
\hline Error(ROOM*SIE*AZTM) & 9.152 & 32 & .286 & & \\
\hline FREQ * SIDE * AZTM & 5.558 & 16 & .347 & .645 & .835 \\
\hline Error(FREQ*SIDE*AZTM) & 34.468 & 64 & .539 & & \\
\hline ROOM * FREQ * SIDE * AZIM & 2.888 & 16 & .180 & .714 & .769 \\
\hline Error(ROOM*FREQ*SIDE*AZIM) & 16.167 & 64 & .253 & & \\
\hline ID * SWE* AZIM & 65.207 & 16 & 4.075 & 5.744 & .000 \\
\hline Error(IID*SIDE*AZIM) & 45.410 & 64 & .710 & & \\
\hline ROOM * IID * SIDE * AZIM & 5.369 & 16 & .336 & 1.851 & .043 \\
\hline Error(ROOM*IID*SIDE*AZIM) & 11.601 & 64 & .181 & & \\
\hline FREQ * IID * SIDE * AZIM & 11.531 & 32 & .360 & 1.184 & .252 \\
\hline ErTor(FREQ*ID*SIDE*AZIM) & 38.959 & 128 & .304 & & \\
\hline $\begin{array}{l}\mathrm{ROOM} * \mathrm{FREQ} * \mathrm{IID} * \mathrm{SDE} * \\
\mathrm{AZIM}\end{array}$ & 8.568 & 32 & .268 & 1.477 & .067 \\
\hline $\begin{array}{l}\text { Error(ROOM*FREQ*II }{ }^{*} \mathrm{SIDE}^{*} \mathrm{~A} \\
\text { ZIM) }\end{array}$ & 23.205 & 128 & .181 & & \\
\hline
\end{tabular}




\section{CHAPTER 7: BIBLIOGRAPHY}

Blauert, J (1983). Evaluating non-identical ear input signals. In Blauert J, Spatial Hearing, MTT Press, Cambridge.

Blauert, J (1971). Localization and the law of the first wave front in the median plane. Journal of the Acoustical Society of America, 50, 466-470.

Burkhard, MD, Sachs, RM (1975). Anthropometric manikin for acoustic research. Journal of the Acoustical Society of America, 58, 214-222.

Butler, RA, Humanski, RA, Musicant, AD (1990). Binaural and monaural localization of sound in two-dimensional space. Perception, 19, 241-256.

Byrne, D, Dermody, P (1975). Localization of sound with binaural body-worn hearing aids. British Journal of Audiology, 9, 107-115.

Emmerich, DS, Harris, J, Brown, WS, Springer, SP, (1988). The relationship between auditory sensitivity and ear asymmetry on a dichotic listening task.. Neuropsychologia, 26, 133-143.

Fisher, HG, Freedman, SJ (1968). The role of the pinna in localization. Journal of Auditory Research, 8, 15-26.

Gaik, W (1993). Combined evaluation of interaural time and intensity differences: Psychoacoustic results and computer modeling. Journal of the Acoustical Society of America, 94, 1, 98-110.

Giguere, C, Abel, SM (1993). Sound localization: Effects of reverberation time, speaker array, stimulus frequency, and stimulus rise/decay. Journal of the Acoustical Society of America, 94, 2, 769-776.

Gilkey, RH, Anderson, TR (1997). In Gilkey RH and Anderson, TR, Binaural and Spatial Hearing in Real and Virtual Environments, Lawrence Erlbaum Associates, Mahwah.

Gilkey, RH, Anderson, TR (1995). The accuracy of absolute localization judgments for speech stimuli. Journal of Vestibular Research, 5, 6, 487-497.

Good, MD, Gilkey, RH (1996). Sound localization in noise: The effect of signal-to-noise ratio. Journal of the Acoustical Society of America, 99, 2, 1108-1117.

Grantham, DW (1996). Left-right asymmetry in the buildup of echo suppression in normal-hearing adults.. Journal of the Acoustical Society of America, 99, 2, 1118 1123.

Haas, H (1951). Uber den Einfluss des Einfachechos auf die Horsamkeit von Sprache. Acustica, 1, 49-58. 
Hafter, ER, De Maio, J (1975). Difference thresholds for interaural delay. Journal of the Acoustical Society of America, 57, 1, 181-187.

Hartmann, WM (1983). Localization of sound in rooms. Journai of the Acoustical Society of America, 74, 5, 1380-1391.

Hartmann, WM (1997). Listening in a room and the precedence effect. 191-210. In Gilkey, RH and Anderson, TR, Binaural and Spatial Hearing in Real and Virtual Environments, Lawrence Erlbaum Associates, Hillsdale, NJ.

Henning, GB (1974). Detectability of interaural delay in high-frequency complex waveforms. Journal of the Acoustical Society of America, 55, 1, 84-90.

Henning, GB (1974). Lateralization and the binaural masking level difference. Journal of the Acoustical Society of America, 55, 6, 1259-1262.

Jongkees, LBW, Veer, RAVD (1958). On directional sound localization in unilateral deafness and its explanation. Acta Oto-Laryngologica, 49, 119-131.

Klumpp, RG, Eady, H R (1956). Some measurements of interaural time difference thresholds. Journal of the Acoustical Society of America, 28, 5, 859-860.

Kuhn, GF (1977). Model for the interaural time differences in the azimuthal plane. Journal of the Acoustical Society of America, 62, 1, 157-167.

Kuhn GF (1987). Physical Acoustics and Measurements Pertainng to Directional Hearing. 3-25. In Yost, WA \& Gourevitch, G, Directional Hearing, SpringerVerlag, New York.

Loomis, JM, Hebert, C, Cicinelli, JG (1990). Active localization of virtual sounds. Journal of the Acoustical Society of America, 88, 4, 1757-1764.

Makous, JC, Middlebrooks, JC (1990). Two-dimensional sound localization by human listeners. Journal of the Acoustical Society of America, 87, 5, 2188-2200.

Markides, A (1977). Binaural Hearing Aids, Academic Press, New York.

Middlebrooks, JC (1992). Narrow-band sound localization related to external ear acoustics. Journal of the Acoustical Society of America, 92, 5, 2607-2624.

Middlebrooks, JC, Green, DM (1990). Directional dependence of interaural envelope delays. Journal of the Acousticai Society of America, 87, 5, 2149-2162.

Middlebrooks, JC, Makous, JC, Green, DM (1989). Directional sensitivity of soundpressure levels in the human ear canal. Journal of the Acoustical Society of America, 86, 1, 89-108. 
Mills, AW (1958). On the minimum audible angle. Journal of the Acoustical Society of America, 30, 4, 237-246.

Mueller, HG and Hall, JW (1998). Audiologists' Desk Reference, Volume II, Singular Publishing Group, San Deigo, CA.

Musicant, AD, Butler, RA (1985). Influence of monaural spectral cues on binaural localization. Joumal of the Acoustical Society of America, 77, 1, 202-208.

Musicant, $\mathrm{AD}$, Butler, RA (1984). The influence of pinnae-based spectral cues on sound localization. Journal of the Acoustical Society of America, 75, 1195-1200.

Noble, W (1987). Auditory localization in the vertical plane: Accuracy and constraint on bodily movement. Journal of the Acoustical Society of America, 82, 5, 16311636.

Oldfield, SR, Parker, SPA (1986). Acuity of sound localization: A topography of auditory space. III. Monaural hearing conditions. Perception, 15, 67-81.

Pollack, I, Rose, M (1967). Effects of head movements on the localization of sounds in the equatorial plane.. Perception and Psychophysics, 2, 591-596.

Rakerd, B, Hartmann, WM (1985). Localization of sound in rooms II: The effects of a single reflecting surface. Journal of the Acoustical Society of America, 78, 2, $524-$ 533.

Rayleigh, Lord (JW Strutt, 3rd Baron of Rayleigh) (1907). On our perception of sound direction. Philos. Mag., 13, 214-232.

Sandel, TT, Teas, DC, Feddersen, WE, Jeffress, LA (1955). Localization of sound from single and paired sources. Journal of the Acoustical Society of America, 27, 5 , 842-852.

Shaw, EAG, Vaillancourt, MM (1985). Transformation of sound pressure level from the freefield to the eardrum presented in numerical form. Journal of the Acoustical Society of America, 78, 1120-1123.

Shaw, EAG (1974). Transformation of sound pressure level from the free field to the eardrum in the horizontal plane. Joumal of the Acoustical Society of America, 56, 1848-1861.

Shelton, BR, Searle, CL (1980). The influence of vision on the absolute identification of sound-source position. Perception and Psychophysics, 28, 589-596.

Stevens, SS, Newman, EB (1936). The localization of actual sources of sound. American Journal of Psychology, 48, 297-306. 
Thurlow, WR, Runge, PS (1967). Effect of induced head movements on localization of direction of sounds.. Journal of the Acoustical Society of America, 42, 480-488.

Wallach, H, Newman, EB, Rosenzweig MR (1949). The precedence effect in sound localization. American Journal of Psychology, 62, 315-336.

Ward, WD (1957). Hearing of naval aircraft maintenance personnel. Journal of the Acoustical Society of America, 29, 1289-1301.

Wenzel, EM, Arruda, M, Kistler, DJ, Wightman, FL (1993). Localization using nonindividualized head-related transfer functions. Joumal of the Acoustical Society of America, 94, 1, 111-123.

Wightman, FL, Kistler, DJ (1989). Headphone simulation of free-field listening: I:Stimulus synthesis. Journal of the Acoustical Society of America, 85, 2, 858867.

Wightman, FL, Kistler, DJ (1989). Headphone simulation of free-field listening: II:Psychophysical validation. Joumal of the Acoustical Society of America, 85, 2, 868-878.

Wightman, FL, Kistler, DJ (1992). The dominant role of low-frequency interaural time differences in sound localization. Joumal of the Acoustical Society of America, $91,3,1648-1661$.

Wightman, FL, Kistler, DJ (1997). Factors affecting the relative salience of sound localization cues. 1-23. In Gilkey RH and Anderson, TR, Binaural and Spatial Hearing in Real and Virtual Environments, Lawrence Erlbaum Associates, Mahwah.

Woodworth, RS (1938). Experimental Psychology, Holt, New York.

Yost, WA, Wightman, F L, Green, DM (1971). Lateralization of filtered clicks. Journal of the Acoustical Society of America, 50, 6, 1526-1531.

Zurek, PM (1980). The precedence effect and its possible role in the avoidance of interaural ambiguities. Journal of the Acoustical Society of America, 67, 952-964. 